\title{
Molecular Beam Studies of Energy Transfer in Molecule Surface Interactions
}

\author{
Dissertation \\ for the award of the degree \\ "Doctor of Philosophy" (Ph.D.) \\ Division of Mathematics and Natural Sciences \\ of the Georg-August-Universität Göttingen \\ submitted by \\ Russell James Cooper \\ from The United States of America
}

Göttingen 2011 
Alec Wodtke ( $1^{\text {st }}$ Reviewer)

Jörg Schroeder (2 ${ }^{\text {nd }}$ Reviewer)

Date of the oral examination: 


\section{Abstract:}

Chemical reactivity at metal surfaces is extremely important in modern chemistry. Motivation for a deeper understanding of these processes has led to the development of new fields of study, such as surface science. One of the most important goals of surface science has been a detailed understanding of the energy transfer in gas surface interactions. However, the underlying dynamics, governing energy transfer at solid surfaces, are not well understood.

Translational energy transfer of the molecules to the surface plays an important role, as translational energy loss is fundamental for sticking at the surface, the first step in many chemical reactions. Molecular beam methods, combined with laser spectroscopy, are used in this work to measure the incident and final translation energy of $\mathrm{HCl}$ and $\mathrm{NO}$ scattering from a $\mathrm{Au}(111)$ surface in well-defined quantum states. I measured the dependence of both the incidence vibrational state on the final translational energy distribution, as well as the interplay between vibrational relaxation and the final translational energy. I show that vibrational relaxation increases the final translational energy. I found a substantial fraction of the vibrationally elastically scattered molecules underwent translational energy loss that exceeded the expectation in an impulsive collision, thought to be the limit in a purely mechanical mechanism.

One of the most important and least studied energy pathways, for molecule surface interactions, is the transfer of energy between the vibrational degree of freedom of the molecule and the electronic degrees of freedom in the surface. This pathway is particularly important as the vibrational degree of freedom couples to bond dissociation. Studies of NO scattering from metal surfaces provided some of the first evidence of coupling between molecular vibration and surface electron hole pairs. More dramatically, the multi-quantum vibrational relaxation of $\mathrm{NO}\left(\Delta \mathrm{v}_{\max }=-8\right)$ was seen to couple to a single electron. Multi-quanta vibrational excitation via coupling to electron hole pairs has, however, never been measured. For NO scattering from $\mathrm{Au}(111)$, I measured vibrational excitation probabilities of $\mathrm{NO}(\mathrm{v}=0 \rightarrow 2)$ and $\mathrm{NO}(\mathrm{v}=0 \rightarrow 1)$ at a wide variety of surface temperatures and incident translational energies. I developed a kinetic model to help elucidate the mechanism for multiquantum vibrational excitation, whether direct, $\mathrm{NO}(\mathrm{v}=0 \rightarrow 2)$, or sequential, $\mathrm{NO}(\mathrm{v}=0 \rightarrow 1 \rightarrow 2)$. Multi-quanta vibrational excitation is shown to occur predominately via direct excitation. 


\section{Table of Contents}

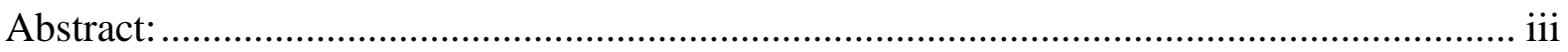

1. Introduction:

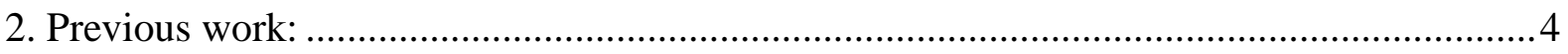

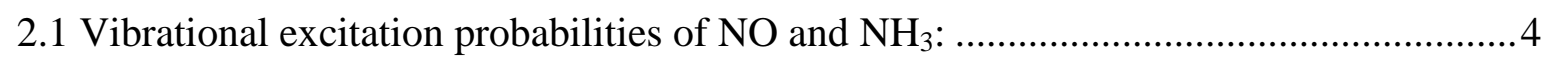

2.2 Theoretical model for vibrational excitation of NO: ................................................ 6

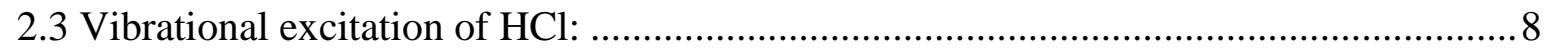

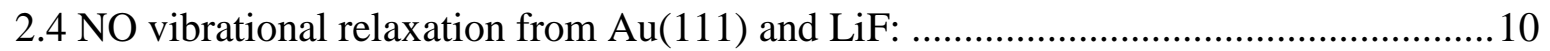

2.5 Theoretical models for NO vibrational relaxation: .................................................... 12

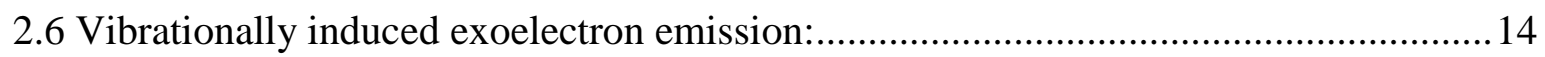

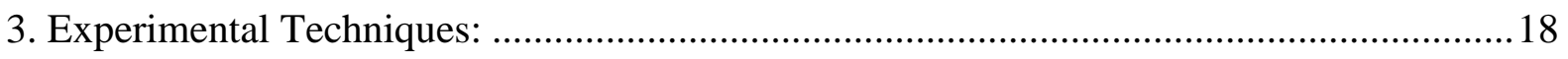

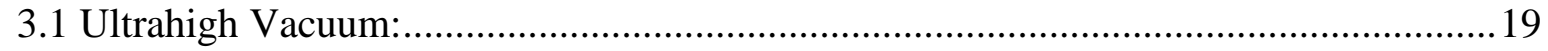

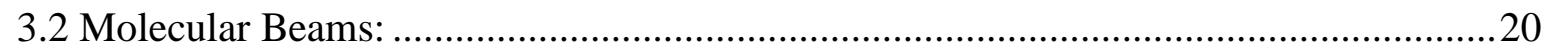

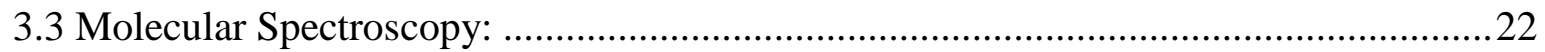

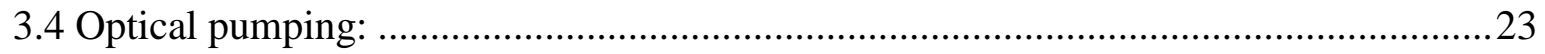

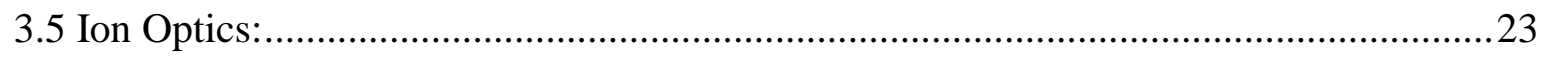

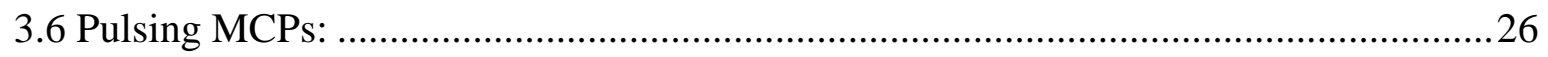

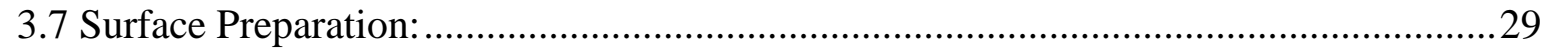

3.8 Experimental details for $\mathrm{HCl}$ translational energy transfer: ....................................... 31

3.9 Experimental details for NO vibrational excitation probabilities: ................................36

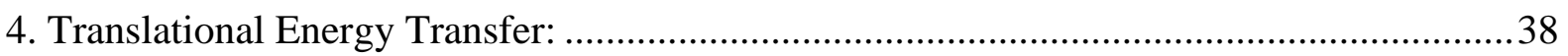

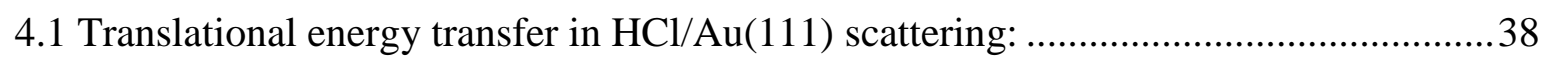

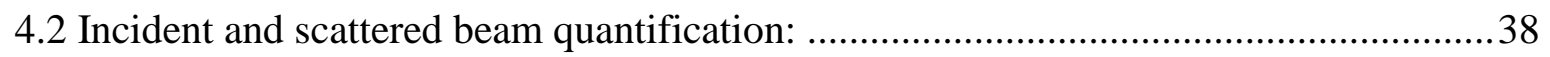

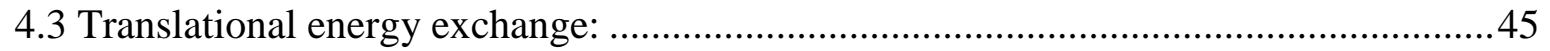

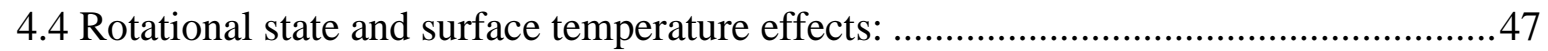

4.5 Coupling of vibrational and translation of a scattered molecule ..................................51

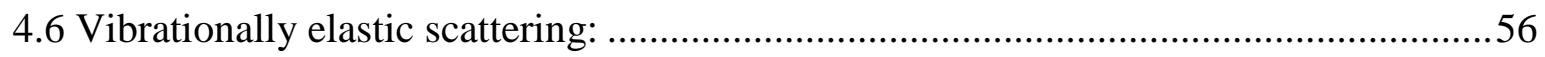

4.7 Comparison of translational inelasticity for $\mathrm{HCl} \mathrm{v}=2$ and $\mathrm{v}=0$ vibrationally elastic

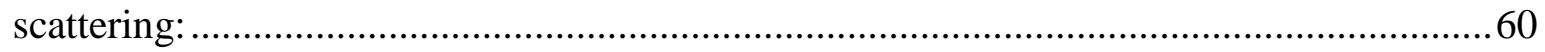

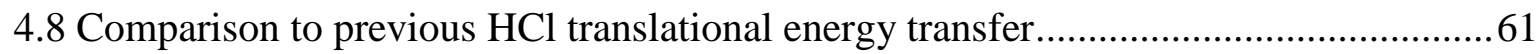

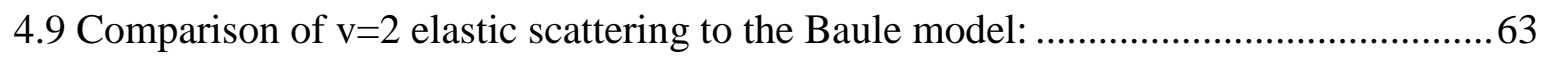

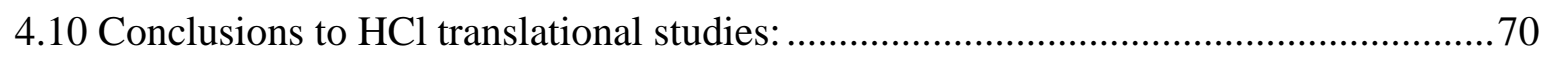




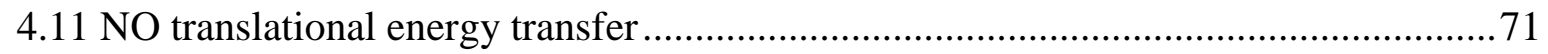

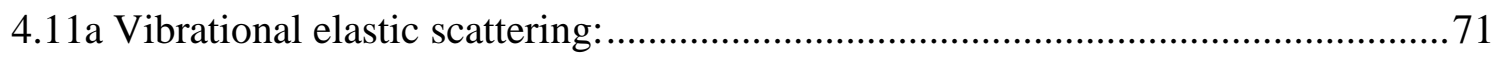

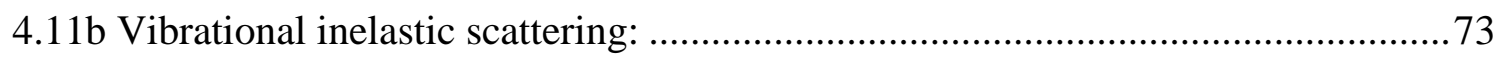

5. Derivation of absolute vibrational excitation probabilities:............................................. 76

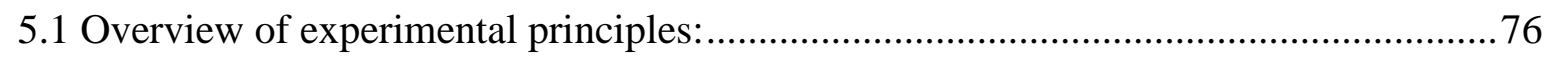

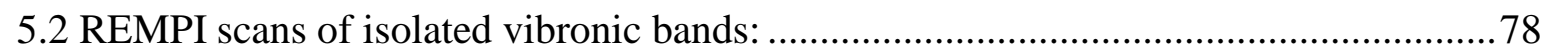

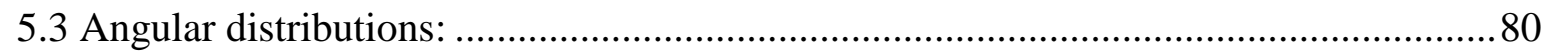

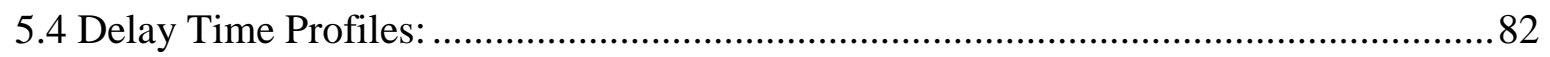

5.5 Laser power dependence, A-state ionization cross-sections, and MCP Gain:................82

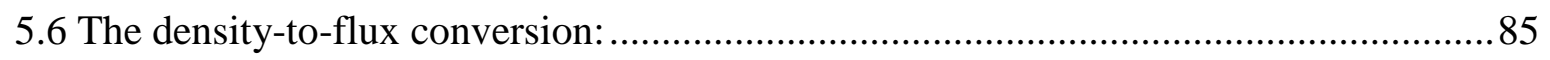

5.7 Conclusions of the derivation of absolute vibrational excitation probabilities: ............85

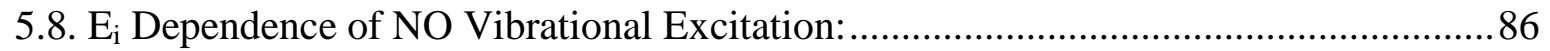

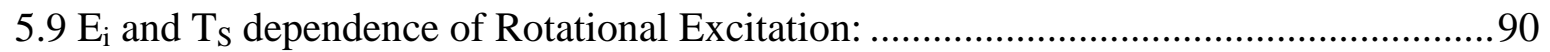

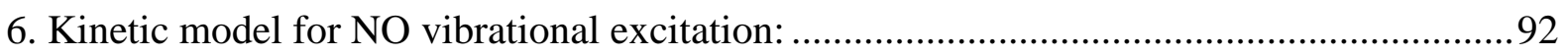

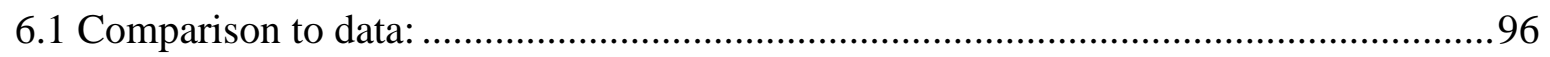

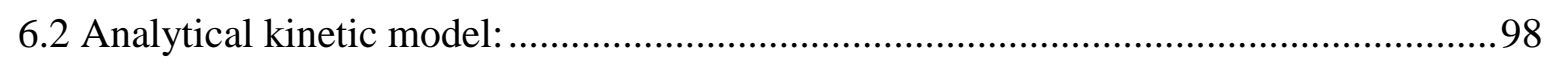

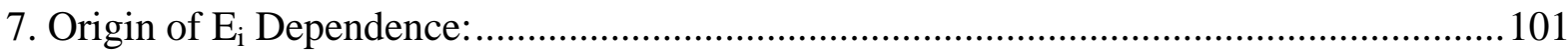

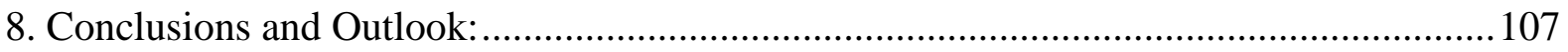

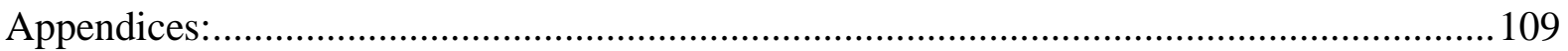

Appendix I: Conversion of TOF data to translational energy distributions: ...................... 109

Appendix II Additional measurements for ion collection efficiency: ............................. 111

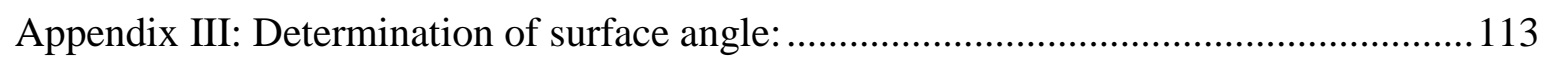

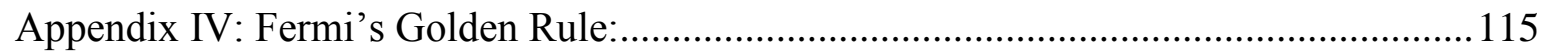

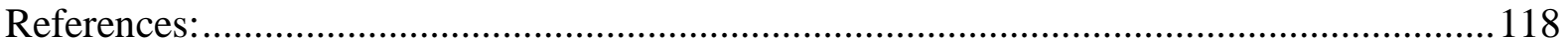

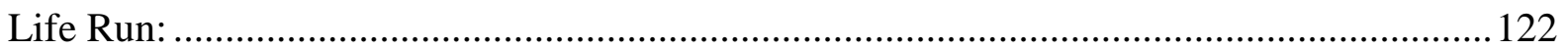

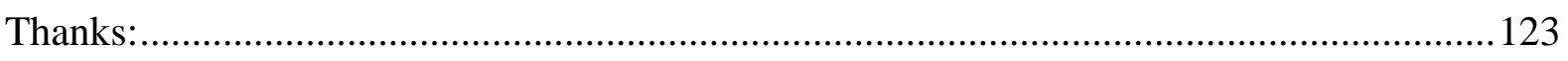




\section{Introduction:}

Chemical reactivity at surfaces plays an important role in the modern industrialized age. Understanding of the interactions between gaseous molecules and solid surfaces is critical from a scientific and technological view. Heterogeneous catalysis is of particular importance due to its importance to a wide range of applications from agro to pharmaceutical, semiconductor, and petrochemical industries. Clearly a better understanding of basic dynamics that govern heterogeneous interactions would be beneficial.

The push for the understanding of molecules at surfaces has led to the development of a number of fields of science such as interfacial chemistry, catalytic chemistry, and surface science. Many of the interactions between molecules and surfaces are similar to those found in gas phase reaction dynamics however, the surface further complicates the dynamics of the interaction. For example the structure of the surface is thought to play a strong role in its reactivity as reactions often occur as step sites or surface anomalies. ${ }^{1}$

The Born-Oppenheimer approximation $(\mathrm{BOA})^{2}$ has been successful in modeling gas phase reaction dynamics. This is due to the fact that the excited electronic states for the molecules are significantly high in energy and the nuclear motion sufficiently slow that molecular scattering is typically electronically adiabatic, that is it occurs on only the lowest potential energy surface (PES). This allows theoreticians to utilize the BOA in modeling the scattering dynamics in gas phase reactions. However, non-adiabatic interactions may be substantially more common in molecule surface interactions than in the gas phase. Evidence for non-adiabatic interactions has been seen for molecules adsorbed on surfaces, ${ }^{3-7}$ as well as molecular beam surface scattering experiments. ${ }^{8-12}$ Despite the fact that non-adiabatic behavior in molecule surface interactions is predominant enough to warrant several reviews, ${ }^{13-19}$ the BOA, perhaps due to a lack of alternatives, has traditionally been utilized in modeling gas surface reactions, where it can be successful depending on the system in study. ${ }^{20-23}$

Most of the interactions between surfaces and gas molecules can be described in the following processes: ${ }^{24}$ 

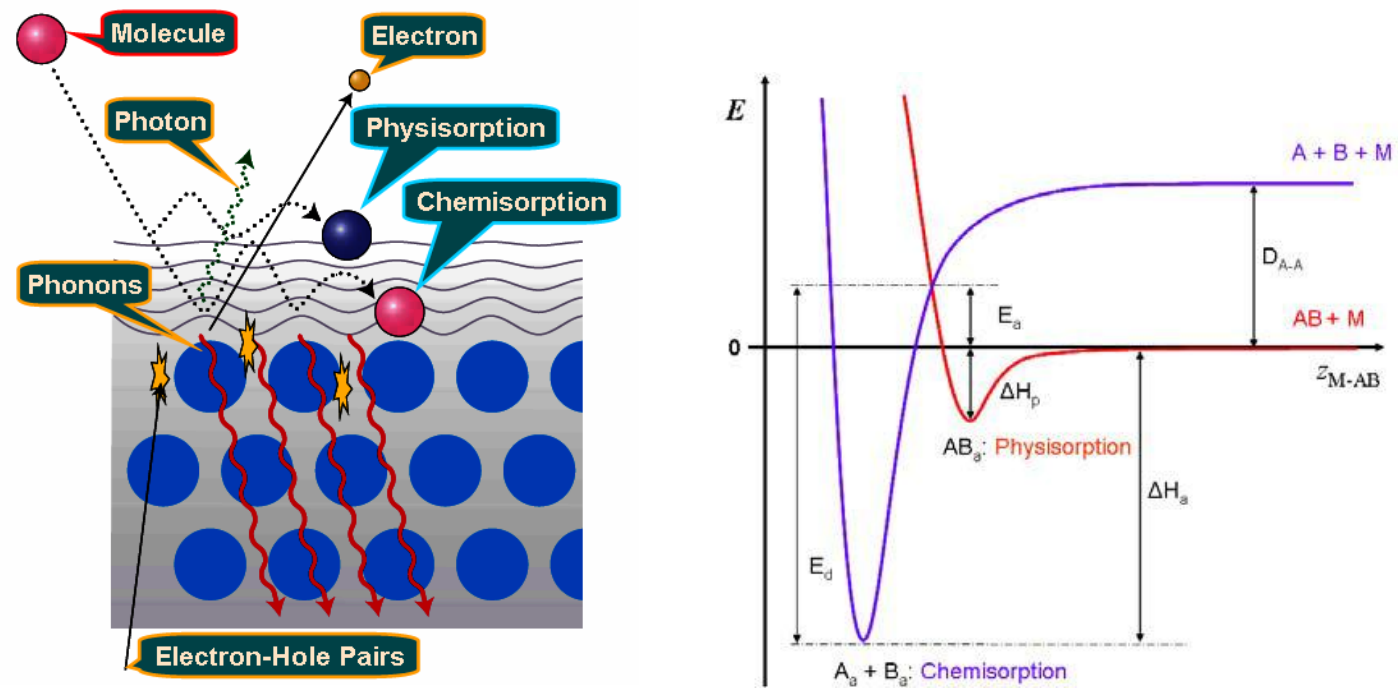

Figure 1.1 Left panel shows primary interactions that occur in molecule surface scattering. Right panel shows typical chemisorption and physisorption energetic interactions as a function of molecular distance from the surface. ${ }^{24-26}$

Physisorption: the incoming molecule may be trapped in an energetically shallow physisorption state at the surface, see red curve in Fig. 1.1. This happens if the translational energy of the atom or molecule is efficiently dissipated to the surface. Physisorption forces are usually of van der Waals nature, with corresponding binding energies typically less than $0.2 \mathrm{eV}$.

Chemisorption: if the molecule surface interaction has enough energy to overcome a dissociation barrier of the molecule, or if no barrier to dissociation exists, the molecule can enter into a chemisorption state, see blue curve in Fig. 1.1. In this state atoms or molecules are strongly bound at the surface, and the bonds are usually of covalent or partially ionic nature, and corresponding binding energies are typically on the order of several electron volts. The dissociation barrier of a molecule at the surface is not as clear as in gas phase chemistry as this barrier may be strongly surface site dependent. The trajectory in which the molecule approaches the surface may also strongly alter the dissociation barrier with the surface.

Additionally, once the molecule undergoes physisorption, they may diffuse to other parts of the surface, which may have increased chemical reactivity. This may also lead to ambiguity in the dissociation mechanism, as the molecule could directly dissociate upon collision or first adsorb in a shallow well, diffuse to a site of high reactivity, and then undergo dissociation. ${ }^{27}$ This situation is sometimes called precursor mediated trapping. ${ }^{28}$

Molecule surface interactions involve energy transfer between the molecule and the surface. These interactions can occur in direct scattering, where the molecule does not adsorb on the surface, or when the molecule is adsorbed on the surface. At the energies typical to molecular beams of light diatomic molecules, the main energy exchange at the surface occurs via lattice 
phonons and surface electron hole pairs. The interactions of the surface electron hole pairs with various degrees of freedom of the scattering molecules are the primary topic of this thesis.

It is difficult to measure the energy change in the surface directly, as once a local excitation or relaxation on a metal surface occurs, it is brought back to equilibrium on a ps time scale. In order to accurately measure the energy transfer I choose to probe the energetics of the molecules before and after they interact with the surface. By understanding the overall energy change of the molecules I am able to determine the amount of energy transferred to the surface. Additionally by knowing what degrees of freedom in the molecule exchanged energy with the surface we are able to have some idea of energy transfer process. Variation of the scattering conditions, such as surface temperature or initial kinetic energy of the molecules, also allows for an elucidation of the dynamics between the molecules and the surface.

Here experiments on the role of translational energy of gas molecules coupling to a metal surface will be discussed. Additionally the role of the molecules initial and final vibrational states will be discussed in regards to their effect on the translational energy of the molecule. Multi-quantum vibrational excitation is also measured over a wide range of initial conditions. The mechanism for vibrational excitation is coupling of the vibrational degree of freedom to electron hole pairs in the metal. A kinetic model for vibrational excitation was developed to draw deeper understanding from these results.

The remainder of this thesis is organized as follows. Section 2 describes previous work which is relevant to this thesis. Section 3 provides information on the experimental techniques utilized in carrying out the experiments here, as well as a short background on some of the techniques. Section 4 presents results for translational energy transfer between molecular beams and a $\mathrm{Au}(111)$ surface. Section 5 gives details for the derivation of vibrational excitation probabilities and shows the results for vibrational excitation. Section 6 outlines a kinetic model which is used to explain the surface temperature dependence of the vibrational excitation probabilities. Section 7 has a theoretical explanation of the incidence energy of translation dependence of vibrational excitation. Section 8 contains the conclusions to this work as well as future outlook in this field. 


\section{Previous work:}

\subsection{Vibrational excitation probabilities of $\mathrm{NO}$ and $\mathrm{NH}_{3}$ :}

Molecular beam experiments provided the first direct observation of vibrational excitation in molecule-surface collisions. ${ }^{8,29}$ In this work, NO in its ground vibrational and rotational state was scattered from a $\operatorname{Ag}(111)$ crystal where direct scattering was the predominant dynamic. The scattered molecules were detected state selectively with resonant enhanced multi-photon ionization (REMPI). Vibrational excitation of $\mathrm{NO}(\mathrm{v}=0 \rightarrow 1)$ was measured as a function of incidence energy of translation and surface temperature. The vibrational excitation probability was strongly dependent on incidence energy of translation and surface temperature as seen in Fig. 2.1(A and B).

A few years later vibrational excitation for a direct scattering process was measured in another system, $\mathrm{NH}_{3}$ scattering from $\mathrm{Au}(111) .{ }^{30}$ Vibrational excitation probabilities for $\mathrm{NH}_{3}(\mathrm{v}=0 \rightarrow 1,2,3)$ were measured as a function of incidence translational energy and surface temperature. Here, the excitation probabilities for all final vibrational states were shown to be strongly dependent on incidence translational energy with a clear threshold at the energy required for vibrational excitation, but vibrational excitation was independent of surface temperature. 

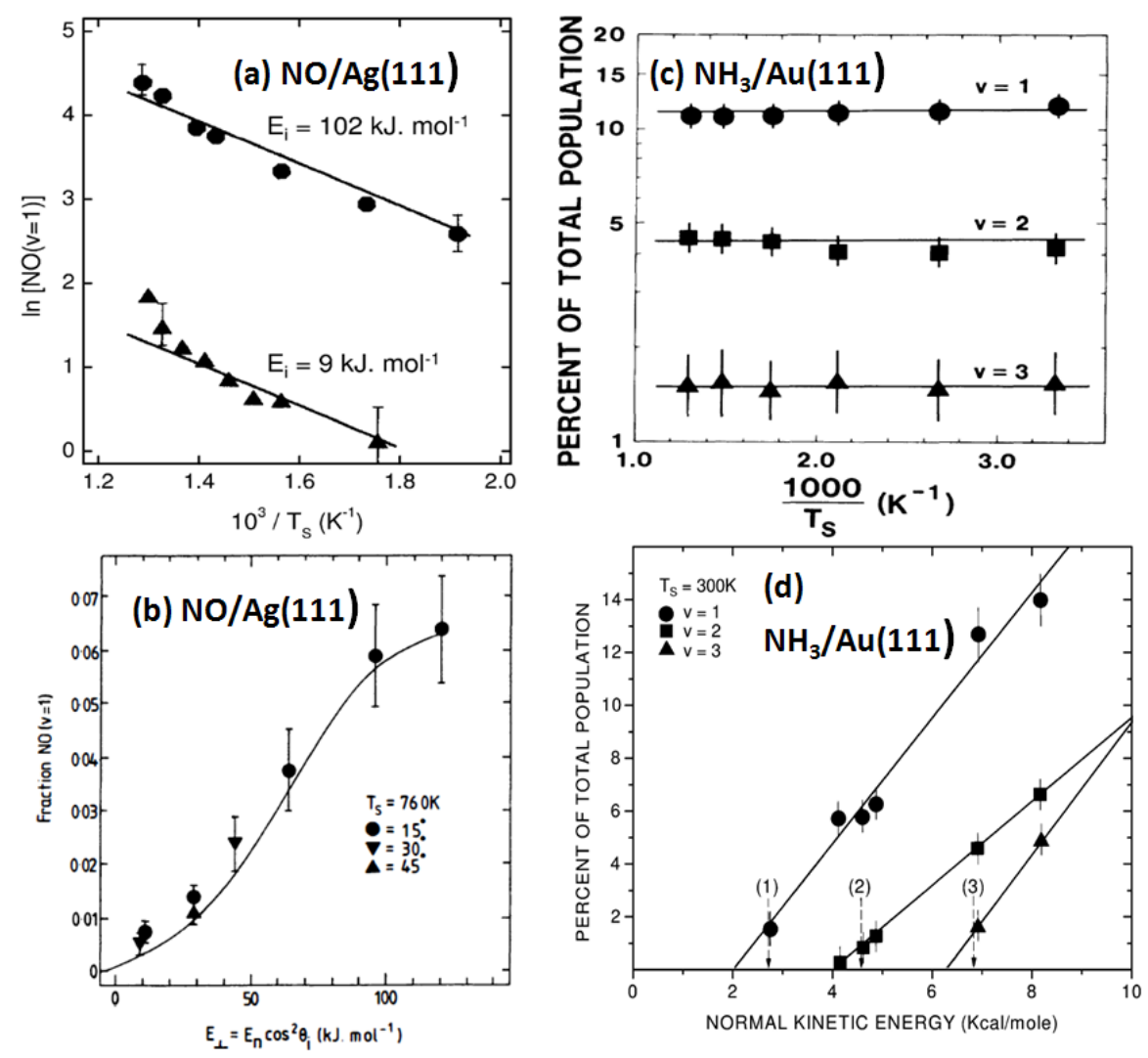

Figure 2.1 A and $\mathrm{C}$ show surface temperature of vibrational excitation for $\mathrm{NO}$ scattering form $\operatorname{Ag}(111)$ and $\mathrm{NH}_{3}$, scattering from $\mathrm{Au}(111)$ respectively. $\mathrm{B}$ and $\mathrm{D}$ show incidence translational energy dependence for $\mathrm{NO}$ scattering form $\mathrm{Ag}(111)$ and $\mathrm{NH}_{3}$, scattering from $\mathrm{Au}(111)$. No threshold is seen in the incidence translational energy dependence for $\mathrm{NO}$ scattering from $\mathrm{Ag}(111)$, however a clear threshold, at the vibrational spacing, exists for $\mathrm{NH}_{3}$ scattering from $\mathrm{Au}(111){ }^{29,30}$

Two different mechanisms were invoked to explain the vibrational excitation in these two systems. NO vibrational excitation was explained via electronically non-adiabatic coupling between the vibration of the molecule and electrons in the metal surface. This electron mediated energy transfer is indicative of a breakdown of the Born-Oppenhiemer approximation. The thermally excited electrons relaxed to holes and this energy was transferred to the vibrational degree of freedom in the molecule. When plotted in Arrhenius fashion, the surface temperature dependence has an activation energy equal to the vibrational spacing of NO. This was one of the first examples of non-adiabatic coupling in molecule surface interactions. The shape of this dependence arises due to the statistical distribution of thermally excited electrons in the metal, which has an exponential dependence on the surface temperature, with enough energy to excite the vibration in the molecule. While there is a strong incidence translational energy dependence, vibrational excitation occurs even at incidence energies substantially smaller than that of the vibrational spacing. Additionally no threshold in the incidence translational energy is observed. The increase in vibrational excitation at increased incidence energy of translation is explained by a stronger coupling between the vibration of the molecule and the thermally excited electrons in 
the surface as the molecule penetrates deeper into the electron cloud of the metal. It is assumed that the translational energy does not couple directly to vibrational excitation.

$\mathrm{NH}_{3}$ vibrational excitation was explained via a mechanical mechanism. Here the incidence translational energy of the molecule is coupled to its vibration upon scattering from the surface. This purely mechanical mechanism gives rise to the surface temperature and incidence energy of translation dependence. Since the energy for vibrational excitation comes from the incidence translational energy in the molecules, changing the surface temperature does not significantly increase the energy of the collision so there is no surface temperature dependence for vibrational excitation. The clear threshold for vibrational excitation seen in the incidence energy of translation dependence indicates that only when the incidence energy of translation exceeds the vibrational threshold can vibrational excitation take place. As incidence energy increases beyond the vibrational spacing of the molecule there is an increased probability for the incidence energy of translation to be coupled to the vibration of the molecule.

\subsection{Theoretical model for vibrational excitation of NO:}

Perhaps the first attempt to model electronically non-adiabatic effects in surface scattering was the work of Newns, ${ }^{31}$ although later other groups used an adaptation of these ideas for other systems. ${ }^{32}$ Here, Newns used a perturbative theory to explain the experimentally observed vibrational excitation of NO scattered from $\operatorname{Ag}(111)$, seen in the previous section. Newns used a similar model to one used to model vibrational relaxation of vibrationally excited molecules adsorbed on metal surfaces. ${ }^{33,34}$ Here the driving force of the vibrational energy change is an electron transfer from the surface to the NO molecule. The electron affinity of the NO decreases in energy as the molecule approaches the surface as the transient ion is stabilized by its image charge on the surface. Additionally, the affinity level is lifetime broadened as it approaches the surface. This allows an electron from the surface to jump to the molecule and upon its return to the surface, it may occupy a different energy level. If the energy spacing between original and final energy of the electron is equal to the vibrational energy spacing in the NO molecule then the vibrational quantum state can change.

In this model the coupling of the electrons of the surface to the NO molecule varies with time. This time is a function of surface distance, $z$, and the velocity of the molecule. The surface molecule interaction is approximated by using a reaction coordinate of a single dimension in which the NO/surface interaction is governed by a simple Morse potential which varies as a function of surface distance:

$$
U(z)=D_{0}\left(e^{-2 \alpha z}-2 e^{-\alpha z}\right)
$$

where $D_{o}$ is the well depth and $\alpha$ is the width of the well. The stabilization of the affinity level is given by: 


$$
E(z)=E_{\infty}-\frac{e^{2}}{4\left(z-z_{0}\right)}, \quad E_{\infty}=\Phi-E_{a}
$$

where the zero of energy is the Fermi level and $\Phi$ and $E_{a}$ provide the work function of the surface and electron affinity of the molecule, respectively.

The broadening of the affinity level is given by:

$$
\Delta(z)=\Delta_{0} e^{-2 \alpha z}
$$

where $\Delta_{0}$ is adjusted for a best fit to the data. Combining the broadening and energy of the affinity level allows for a calculation of the projected density of states (PDOS), which is essentially the interaction region where vibrational excitation occurs. The equation for the PDOS is:

$$
\rho(z)=\frac{1}{\pi} \frac{\Delta(z)}{\Delta(z)^{2}+E(z)^{2}}
$$

The combination of the molecular trajectory and the PDOS gives the overall interaction strength, and consequently the vibrational excitation.

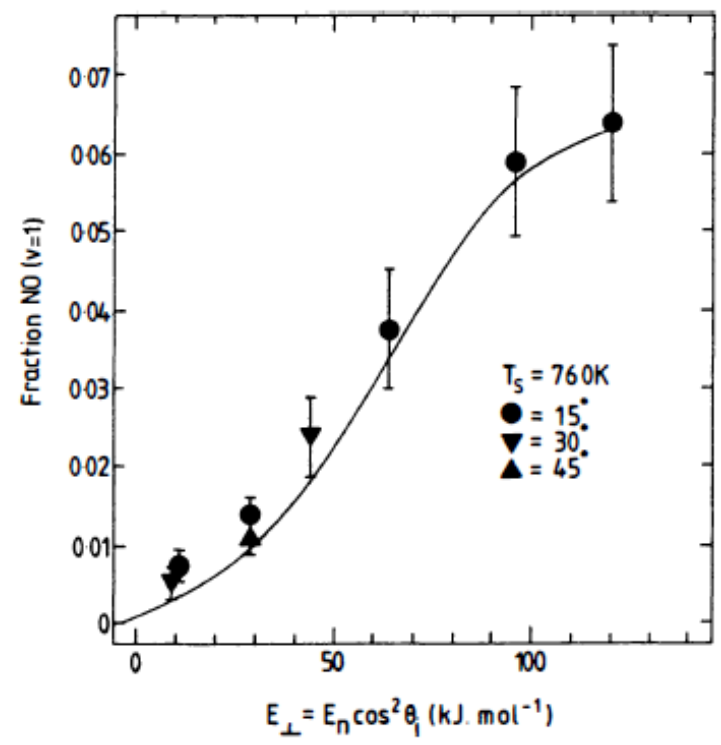

Figure 2.2 Fit of Newn's model to vibrational excitation of NO scattered from $\mathrm{Ag}(111)$ measured as a function of the normal component of the incidence energy of translation. ${ }^{31}$

A more in depth explanation of the kinetic energy dependence according to the formalism shown here is carried out later in this thesis. 


\subsection{Vibrational excitation of $\mathbf{H C l}$ :}

In section 2.1 two mechanisms for vibrational excitation were shown. Perhaps the more complicated of the two is the coupling of the NO vibrational degree of freedom to electrons in the metal. This indicates a breakdown of the Born-Oppenhiemer approximation, which consequently means many commonly used theoretical methods are not sufficient for accurate modeling of NO metal interactions. It is important to understand how general this electronically non-adiabatic coupling between metal surfaces and impinging gas molecules is. Wodtke and coworkers studied vibrational excitation of $\mathrm{HCl}$ scattering from a $\mathrm{Au}(111)$ surface in an effort to better understand how general is this phenomenon. ${ }^{12,35}$ In this study molecular beams of $\mathrm{HCl}$ in its ground rotational and vibrational state were scattered from $\mathrm{Au}(111)$. The molecules were detected state-selectively using REMPI. From angular distributions, temporal profiles, and rotational temperatures the authors determined the scattering occurred in a direct mechanism and trapping was not significant at the incidence translational energies studied in that work. Vibrational excitation probabilities were measured as a function of both surface temperature and incidence energy of translation. As shown in section 2.1, vibrational excitation occurring in a mechanical mechanism should show no surface temperature dependence and the incidence energy of translation dependence should have a clear threshold. If coupling between vibration of the molecule and electrons of the metal is the dominant process, an Arrhenius temperature dependence and an incidence energy of translation dependence with no clear threshold should be seen. Fig. 2.3 shows Arrhenius plots for $\mathrm{HCl}(\mathrm{v}=0 \rightarrow 1)$ vibrational excitation at different incidence energies of translation. 


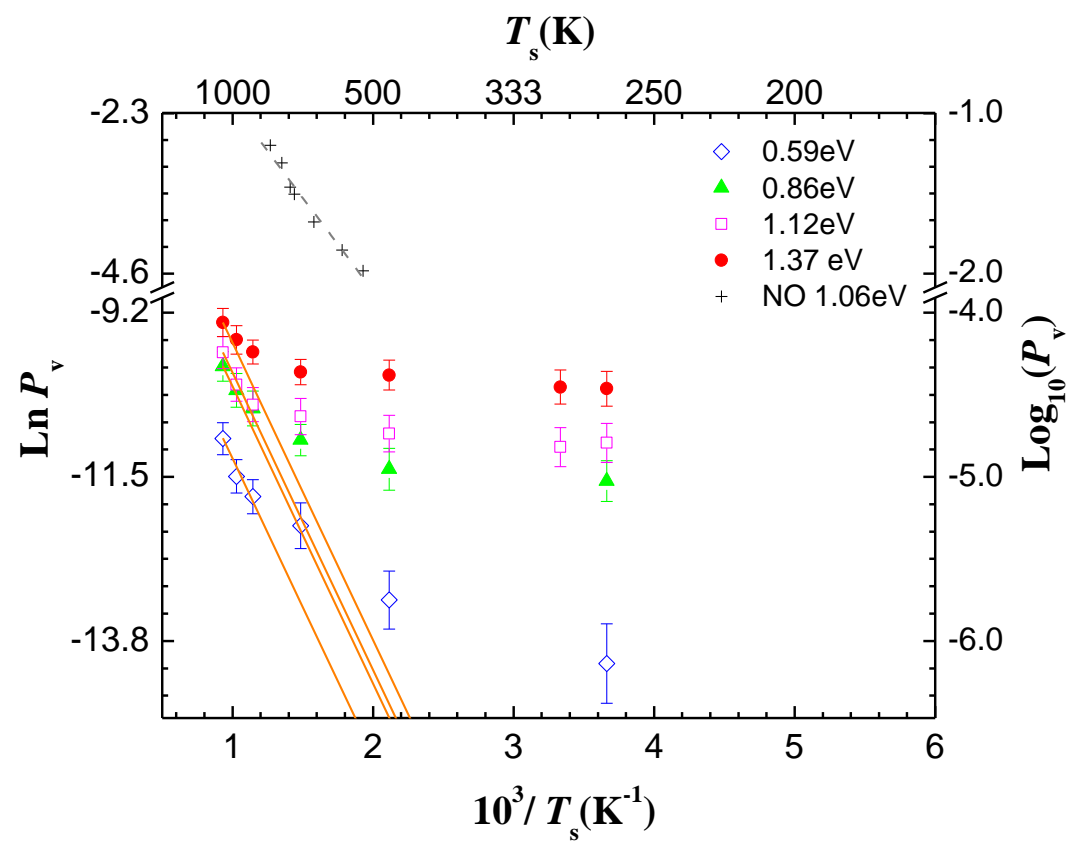

Figure 2.3 Vibrational excitation probability of $\mathrm{HCl}(\mathrm{v}=0 \rightarrow 1)$ scattered from $\mathrm{Au}(111)$ shown as a function of surface temperature at four incidence energies of translation. Vibrational excitation probabilities of NO are also shown. ${ }^{8}$ The Arrhenius dependencies with the expected activation energy fixed at the $\mathrm{HCl}(\mathrm{v}=0 \rightarrow 1)$ energy spacing, $0.36 \mathrm{eV}$, are shown as solid lines. The Arrhenius dependence of $\mathrm{NO}$ with an activation energy of $0.23 \mathrm{eV}$ is shown as a dashed line. $^{12}$

The temperature dependence seen in Fig. 2.3 shows $\mathrm{HCl}$ vibrational excitation has an Arrhenius dependence at high surface temperatures, and is almost temperature independent at lower surface temperatures. This indicates that $\mathrm{HCl}$ vibrational excitation occurs via a combination of two mechanisms, mechanical and electronically non-adiabatic. The relative strength of the two mechanisms depends on the scattering conditions. It is likely that $\mathrm{NO}$ vibrational excitation is also a combination of two mechanisms, but that the non-adiabatic component is by far the more dominant mechanism and any vibrational excitation occurring via the mechanical mechanism is masked by the electronic non-adiabatic excitation. The authors of this work used a combination of the two mechanisms to fit the temperature dependence of vibrational excitation. They used a constant value, the contribution from the mechanical mechanism, plus an Arrhenius equation, the contribution from the non-adiabatic mechanism, with the activation energy set to the vibrational spacing. These fits are shown in Fig. 2.4. 


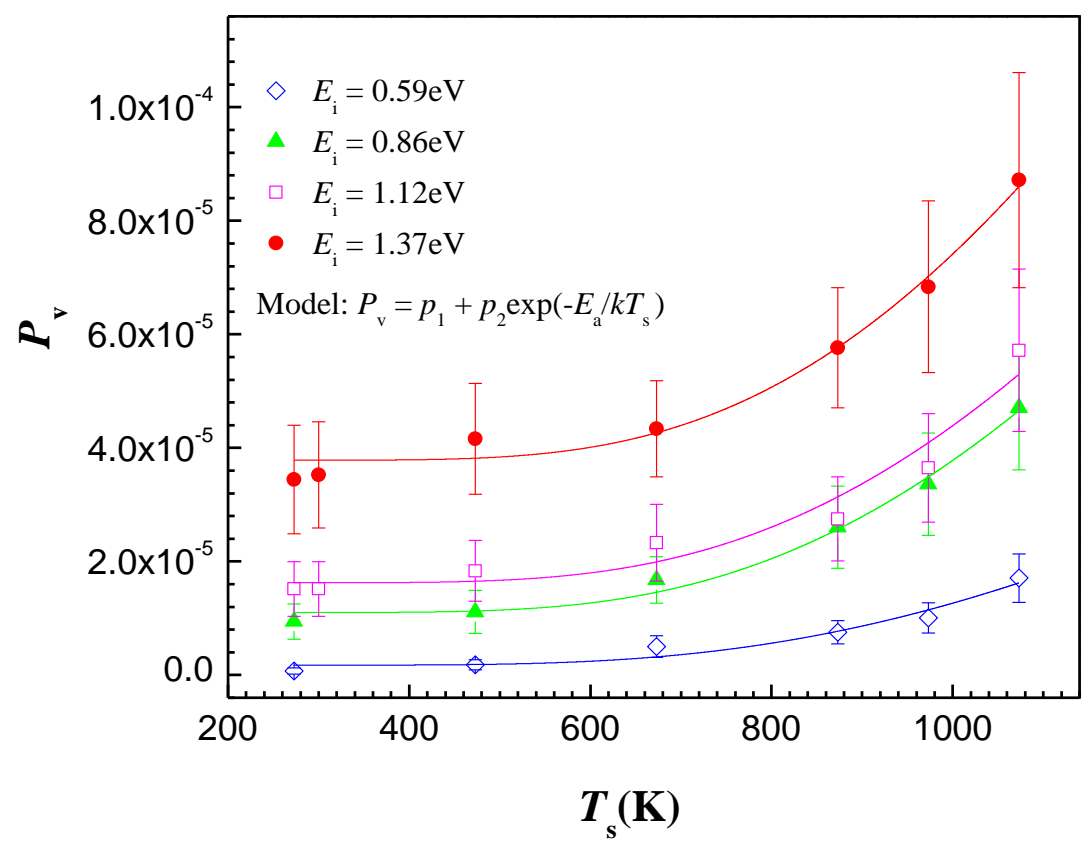

Figure 2.4 Vibrational excitation of $\mathrm{HCl}(\mathrm{v}=0 \rightarrow 1)$ shows a combination of two mechanisms. The symbols are the experimental measurements, of vibrational excitation probability, shown in Fig. 2.3 but plotted as $P_{v}$ vs temperature. The different colors represent different incidence energies. The solid curves are fits from an equation which combines adiabatic and non-adiabatic mechanisms. ${ }^{12}$

The combination of mechanical and electronically nonadiabatic mechanisms fits this data well. These figures along with incidence energy of translation dependence measurements led the authors to conclude that both mechanisms play a prominent role in the vibrational excitation of $\mathrm{HCl}$.

\subsection{NO vibrational relaxation from $\mathrm{Au}(111)$ and $\mathrm{LiF}$ :}

One of the most interesting studies undertaken was the scattering of highly vibrationally excited NO from surfaces of a metal, $\mathrm{Au}(111)$, and an insulator, LiF. ${ }^{10}$ Here highly vibrationally excited molecules were prepared via stimulated emission pumping. These molecules were scattered from the $\mathrm{Au}(111)$ or LiF surface and detected state selectively. The results are shown in Fig. 2.5. 

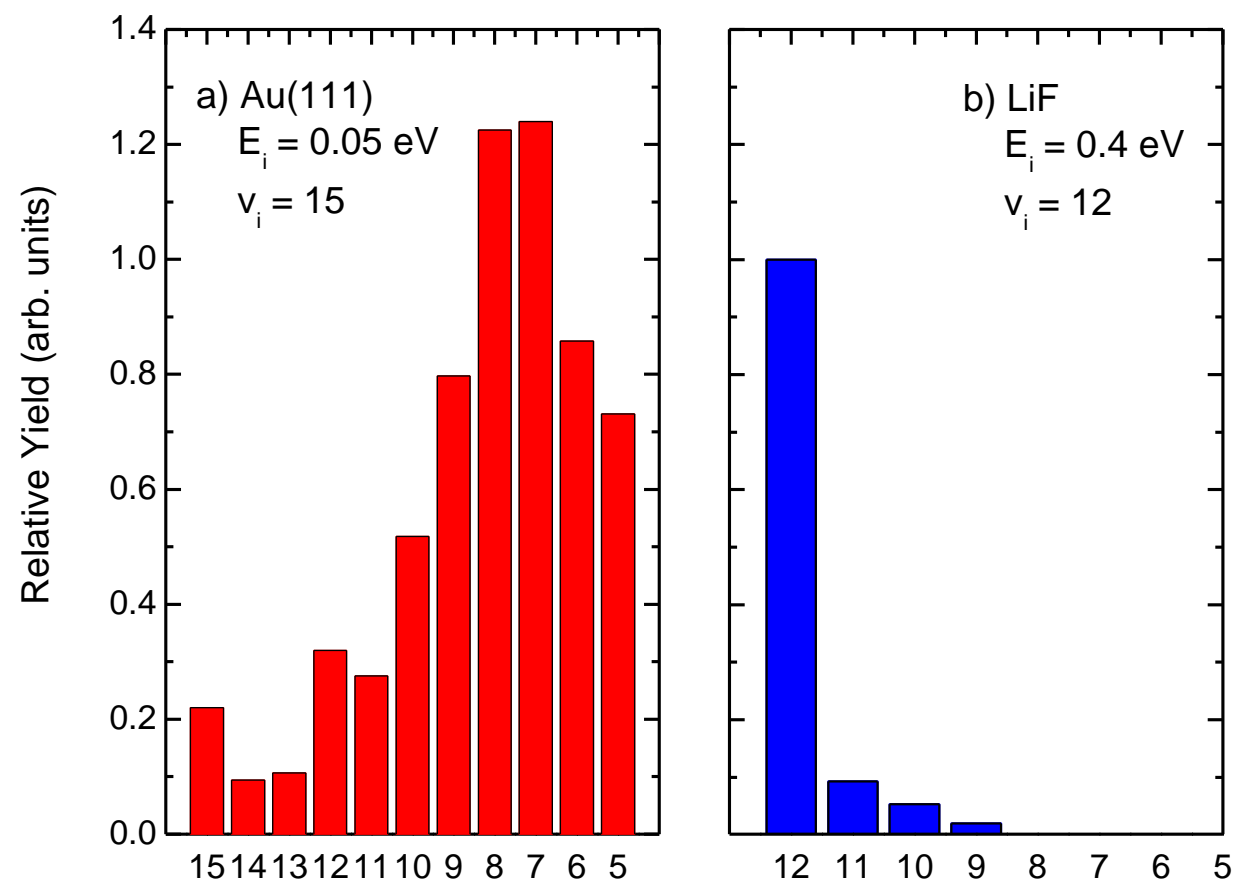

Final Vibrational State

Figure 2.5 Relative final vibrational state distribution for highly vibrationally excited NO molecules scattered from $\mathrm{Au}(111)$, panel a, and LiF, panel b. ${ }^{10}$

The amount of vibrational relaxation when scattering from a metal differs significantly than when scattering from an insulator. The most likely outcome for vibrationally excited molecules interacting with a LiF surface is vibrationally elastic scattering; that is, no vibrational quantum state change is observed. This is in stark contrast to scattering from a metal surface where most molecules undergo multi-quantum vibrational relaxation with $\Delta \mathrm{v}_{\max }=-8$. In both systems the NO undergoes direct specular scattering in sub-ps time scales so the difference in these relaxation probabilities is not caused by a longer time at the surface when NO scatters from Au. These short times combined with the large vibrational energy exchange with the surface suggest that the vibrational energy of the molecule is coupled to electronic excitation in the metal surface. The authors utilized a simple picture to explain these results. Here the vertical electron binding energy varies dramatically, even changing sign, with the length of the bond, and consequently, during the course of molecular vibration, an unoccupied orbital of $\mathrm{NO}$ accepts an electron from the metal surface at the outer turning point of the vibration isoenergetically. As the molecule compresses the potential energy of the ion is raised. The electron then returns to the metal surface at the inner turning point, resonantly. This results in a conversion of the vibrational energy of the molecule to the excitation of a single surface electron. This simple model has been examined in a few theoretical studies ${ }^{36-38}$ and has been utilized to explain dissociative absorption 
of $\mathrm{N}_{2}$ on $\mathrm{Ru}(0001) .{ }^{39}$ In many ways it is analogous to the mechanism invoked to explain NO vibrational excitation when scattered from $\operatorname{Ag}(111){ }^{8}$

\subsection{Theoretical models for NO vibrational relaxation:}

If the vibrational relaxation of NO molecules occurs via coupling between the electrons of the metal and the vibrational degree of freedom of the molecule, then theoretical treatment utilizing adiabatic potentials will clearly fail. Several methods have been developed to address this problem. Two particular examples will be discussed here. The main difference between these two methods is the implementation of the coupling between the electronic degree of freedom in the surface and vibrations of the molecule. The first uses multi-quanta transitions, which are modeled by involving multiple potential energy surfaces and Hamiltonians. The other, electronic friction, uses single quantum transitions where this coupling is modeled with a perturbative treatment of weak electron-vibration coupling.

Independent electron surface hopping (IESH) was developed to model the interaction between NO and the (111) surface of a Gold crystal. This model was created in three steps: 1) detailed $a b$ initio calculations to create multiple potential energy surfaces for the adsorbate/surface system, 2) fitting the $a b$ initio data with a many electron Newns-Anderson Hamiltonian, 3) using independent electron surface hopping to calculate the coupling of the electrons in the metal and the vibration of the molecule. ${ }^{40,41}$

Utilizing these calculation methods they were able to qualitatively reproduce the results shown in the section 2.4 for vibrational relaxation of NO scattering from $\mathrm{Au}(111)$ and $\mathrm{LiF}$ by having the coupling to electrons turned on and off, respectively. ${ }^{42}$ 


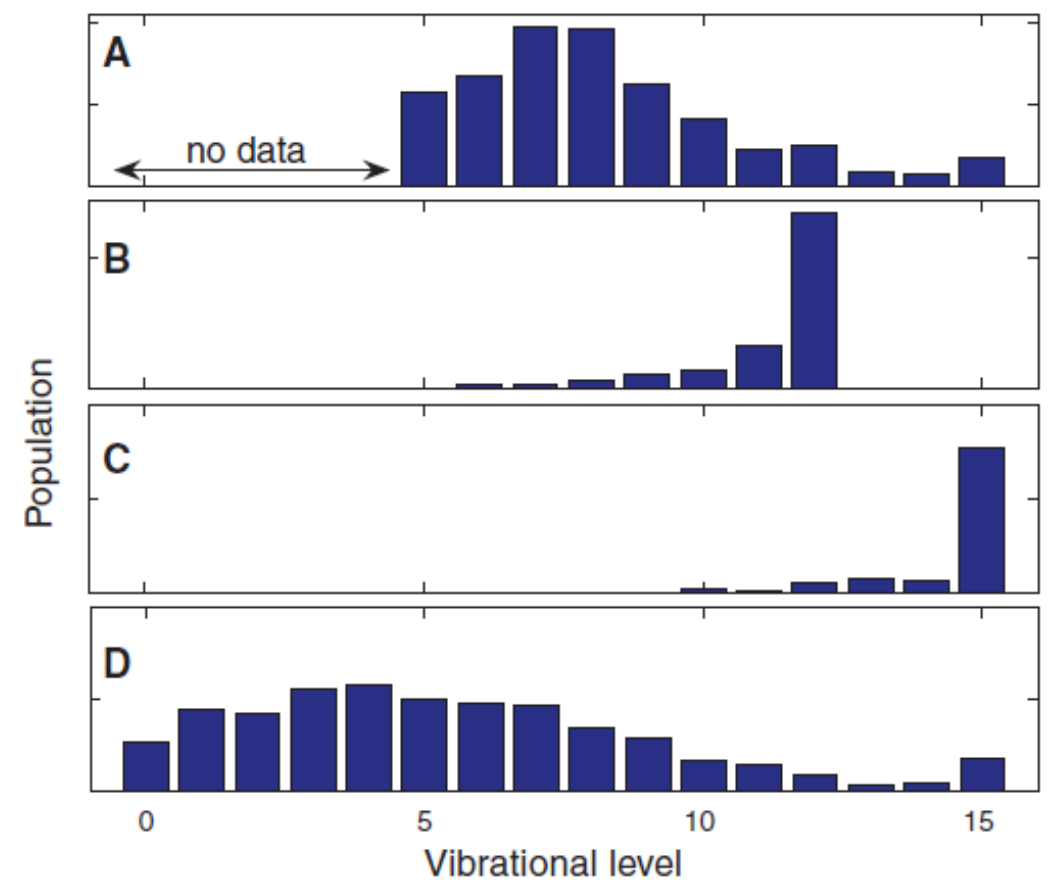

Figure 2.6 NO final vibrational state distribution after scattering from a room temperature surface at surface normal with an incidence energy of $0.05 \mathrm{eV}$. Experimental results are shown in $\mathrm{A}, \mathrm{NO}(\mathrm{v}=15)$ scattering from $\mathrm{Au}(111)$, and $\mathrm{B}, \mathrm{NO}(\mathrm{v}=12)$ scattering from $\mathrm{LiF}$. Theroetical results of $\mathrm{NO}(\mathrm{v}=15)$ scattering from $\mathrm{Au}(111)$ are shown without nonadiabatic coupling, $\mathrm{C}$, and with non-adiabatic coupling, D. ${ }^{42}$

IESH is in relatively good agreement with experimental results. Here the large quantum vibrational relaxation with a broad final state distribution similar to that in experiment is seen. IESH was also able to capture other features of the scattering process such as rotational excitation. It also made several predictions, such as strong orientational effects of the NO molecule on vibrational relaxation. Several of its predictions remain to be tested, but experiments to do so are underway.

The electron coupling to vibrational degrees of freedom can also be modeled indirectly in a fully quantum friction type method. ${ }^{43}$ Due to the cost of fully quantum calculations a two mode, vibrational coordinate and molecule surface distance, single electron potential was used. Coordinate dependent damping rates were calculated for adsorbed species, in good agreement with previous results.

With this information, wavepackets of $\mathrm{NO}(\mathrm{v}=15)$ were propagated towards the surface and the vibrational state is altered via the previously calculated vibrational damping rates. As the wavepacket propagates in a scattering trajectory, the damping rates change as a function of the molecule surface distance. By summing over the time of the scattering event, the final vibrational state distribution can be found. These calculations, of $\mathrm{NO}(\mathrm{v}=15)$ scattered from $\mathrm{Au}(111)$, are made at incidence translational energies higher than that of experiment as lower incidence energies required too large of grids. 


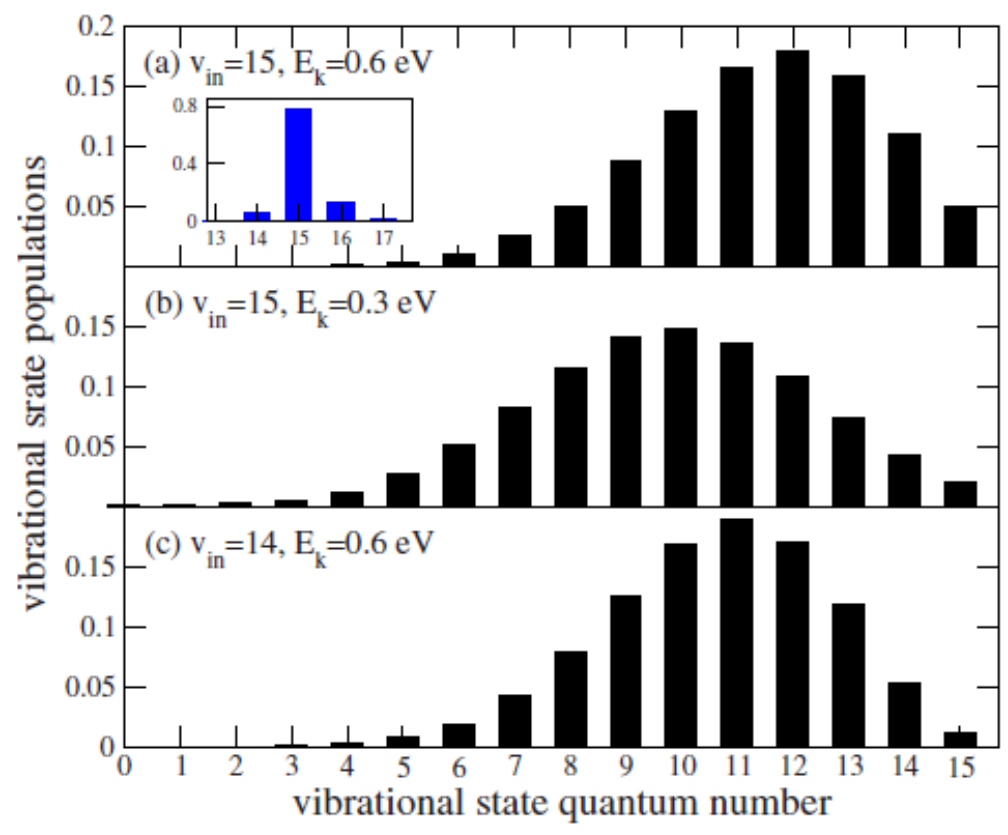

Figure 2.7 Final scattered vibrational state distributions, derived from friction, for two different incidence energies of $\mathrm{NO}(\mathrm{v}=15)$ a) $0.6 \mathrm{eV}$ and $\mathrm{b}) 0.3 \mathrm{eV}$ and $\mathrm{NO}(\mathrm{v}=14) 0.6 \mathrm{eV}$. The inset of a) shows scattering results with the vibrational damping turned off. ${ }^{43}$

The results of these vibrational relaxation measurements show multi-quantum vibrational relaxation with a broad distribution. While the vibrational relaxation is not as large as that in experiment, see Fig. 2.6, the predominant scattering process is multi-quantum relaxation. Furthermore the general trend of lower $\mathrm{E}_{\mathrm{i}}$ resulting in more vibrational relaxation indicates that if this method could be extended to lower $\mathrm{E}_{\mathrm{i}}$ the agreement between experiment and theory would be better. The authors extrapolated their results from the two kinetic energies, and noted that while this was potentially inaccurate, the vibrational energy loss was comparable to that of experiment.

Both of these methods for dealing with electronically non-adiabatic effects of vibrational relaxation in gas surface interactions did a qualitative job of reproducing the experimentally measured final vibrational state distribution of $\mathrm{NO}(\mathrm{v}=15)$ when scattered from a $\mathrm{Au}(111)$ surface. IESH allows for a more complete scattering picture than the friction model and comparison to more degrees of freedom is possible. However for the vibrational degree of freedom, which may be the most important, the simpler friction like calculation also modeled the data quite well.

\subsection{Vibrationally induced exoelectron emission:}

When multi-quantum vibrational relaxation of $\mathrm{NO}$ on $\mathrm{Au}(111)$ was first seen there was some debate as to the mechanism in the relaxation of the NO. ${ }^{10,43}$ While there was no doubt that NO released large amounts of vibrational energy via coupling to electrons in the metal it was unclear whether this happened in a series of sequential single quantum relaxations coupled to multiple 
electrons or via a single relaxation event with all of the energy transferred to a single electron. Theoretical calculations utilizing friction theory were implemented in an attempt to model and interpret the data. ${ }^{43}$ These results showed broad vibrational level distribution after scattering and large average $\Delta \mathrm{v}$ qualitatively similar to the experimental results shown for vibrational relaxation of $\mathrm{NO}(\mathrm{v}=15)$ and the IESH results, where many quanta of vibrational energy were coupled to a single electron, as shown in section $2.5 .^{42}$

Direct evidence of coupling to a single or multiple electrons in the metal was needed to clarify this contested point. A line of reasoning led that if the work function of the metal surface was lower than the vibrational energy of the impinging NO molecule and the vibrational energy of NO was transferred to a single electron, then electrons should leave the surface and be able to be detected. These "exoelectron" experiments were carried out in the Wodtke lab and vibrationally promoted electron emission was seen.

The work function was lowered by decorating the $\mathrm{Au}(111)$ surface with sub-monolayer coverage of Cs. This led to a work function $(\sim 1.6 \mathrm{eV})$ below that of either bulk Au or Cs. ${ }^{44}$ This work function is small enough that the vibrational energy of the $\mathrm{NO}(\mathrm{v}=18)$ molecules excited by stimulated emission pumping is greater than the work function of the surface. This meant that if the vibration of the molecule was to relax with a large $\Delta \mathrm{v}$ and this energy was coupled to a single electron, this electron could escape the surface.

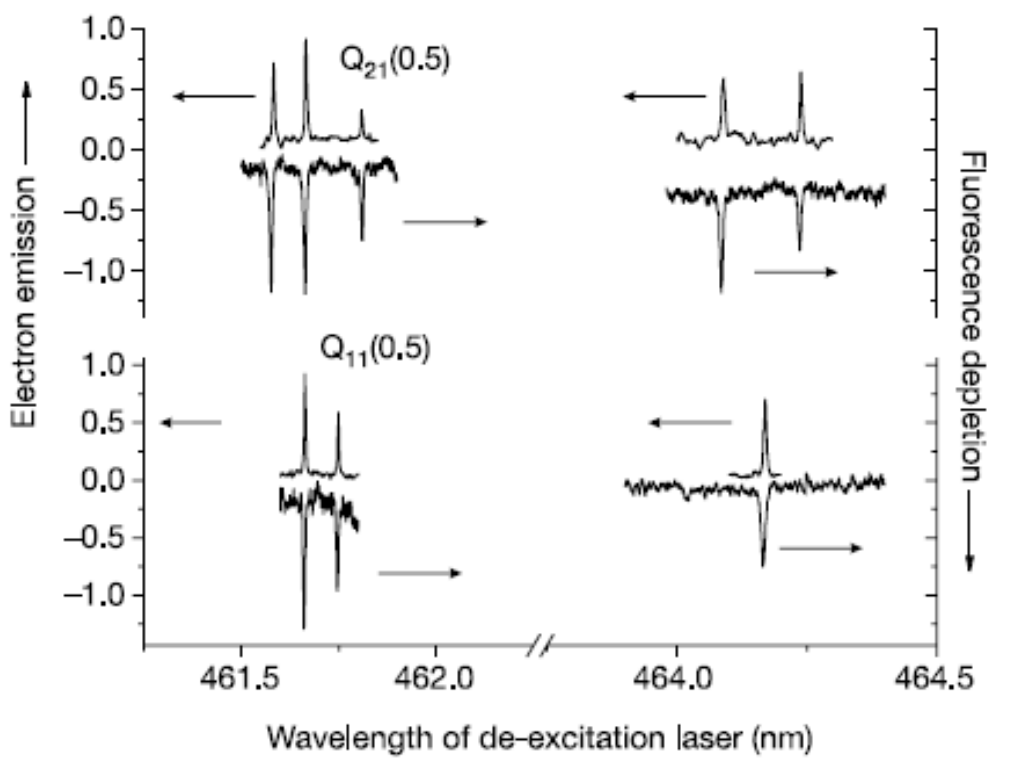

Figure 2.8 The down going signal is the fluorescence depletion signal, indicative of the resonant production of $\mathrm{NO}(\mathrm{v}=18)$. Electron emission from the $\mathrm{Cs} / \mathrm{Au}(111)$ surface. The electron emission is only seen when the $\mathrm{NO}(\mathrm{v}=18)$ is resonantly produced, indicating that electron emission occurs exclusively from highly vibrationally excited molecules. ${ }^{45}$ 
Stimulated emission pumping, similar to that used in the $\mathrm{NO}(\mathrm{v}=15) / \mathrm{Au}(111)$ scattering was employed. ${ }^{45}$ The results in Fig. 2.8 show spectra obtained by tuning one of the lasers (DUMP) used for state preparation while observing the electron signal and fluorescence depletion. The lines seen correspond to dumping to vibrationally excited molecules in the ground state. Electron emission is only strong when the NO is resonantly dumped to a high vibrational state. This shows that electrons are created by vibrational relaxation of NO at the surface, presumably by coupling to a single surface electron. A study of the exo-electron yield as a function of incidence vibrational state of the impinging NO molecules was also undertaken.

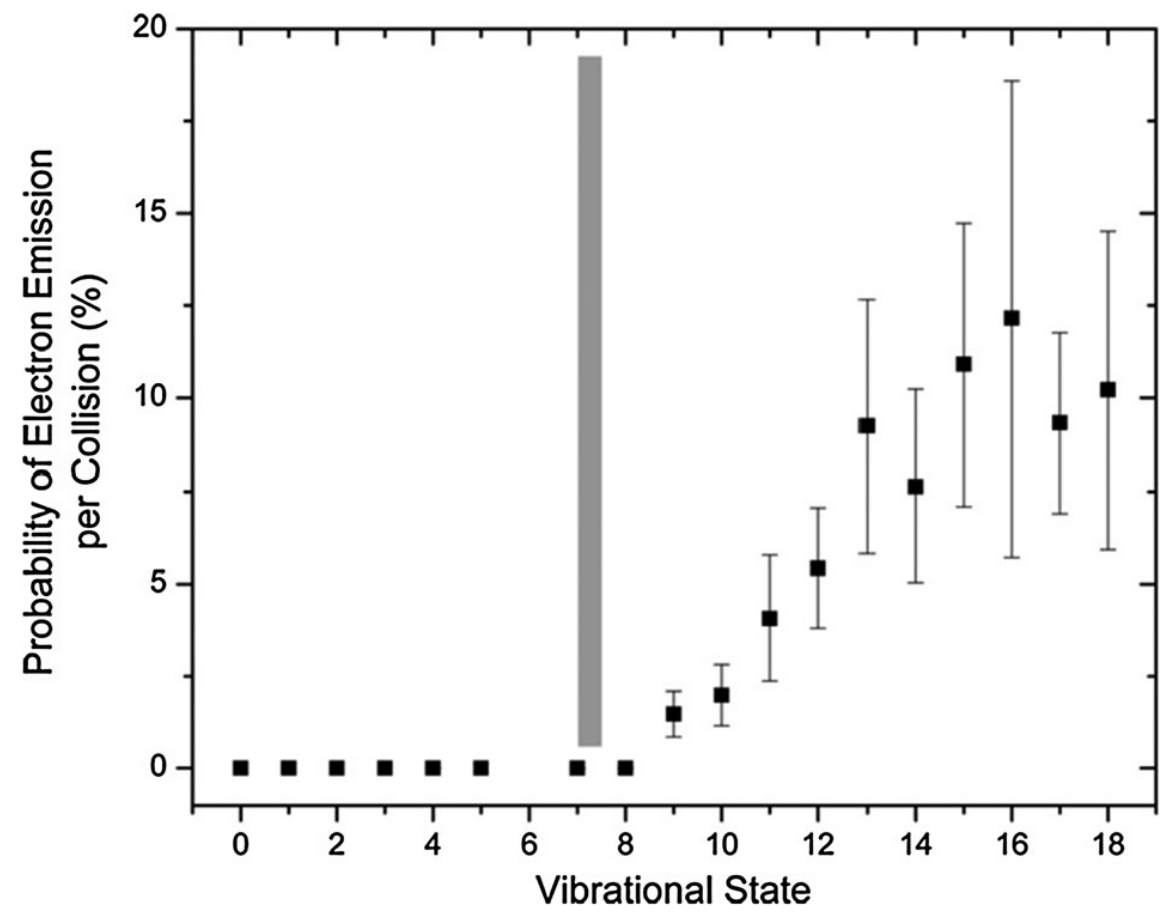

Figure 2.9 Electron emission probability plotted as a function of incoming vibrational state. The grey bar represents the work function of the $\mathrm{Cs}$ decorated Au surface ${ }^{44}$ The original electron emission yield was adjusted upwards after further studies. ${ }^{16,45}$

The electron emission dependence on incidence vibrational energy shows a threshold near the work function of the metal. ${ }^{45}$ The yield increases with increasing vibrational energy up to a point and then levels off. The strong threshold starting at the work function of the surface indicates the vibration couples to a single electron This is strong evidence in favor of the vibrational autodetachment mechanism invoked to describe the $\mathrm{NO}(\mathrm{v}=15)$ relaxation when scattered from $\mathrm{Au}(111)$.

This thesis contains work that probes the importance of electron hole pairs in the metal coupling to molecular degrees of freedom. The next section will describe the experimental techniques utilized in this study. The use and background of a few standard techniques, such as laser spectroscopy, vacuum, and molecular beams will be discussed. I will give a detailed explanation 
of the home-built machine used to carry out this work. These descriptions show both the power and the limitations of the measurements which will be discussed in sections 4-7. 


\section{Experimental Techniques:}

For many years molecule surface scattering has been a technique used to understand the fundamental interactions between gas phase molecules and surfaces. Working in the collisionless regime of high vacuum, with single crystal surfaces creates simple conditions allowing many molecules to scatter from a surface under identical conditions. These simplified conditions allow for comparison to theory, which often is calculated on idealized systems.

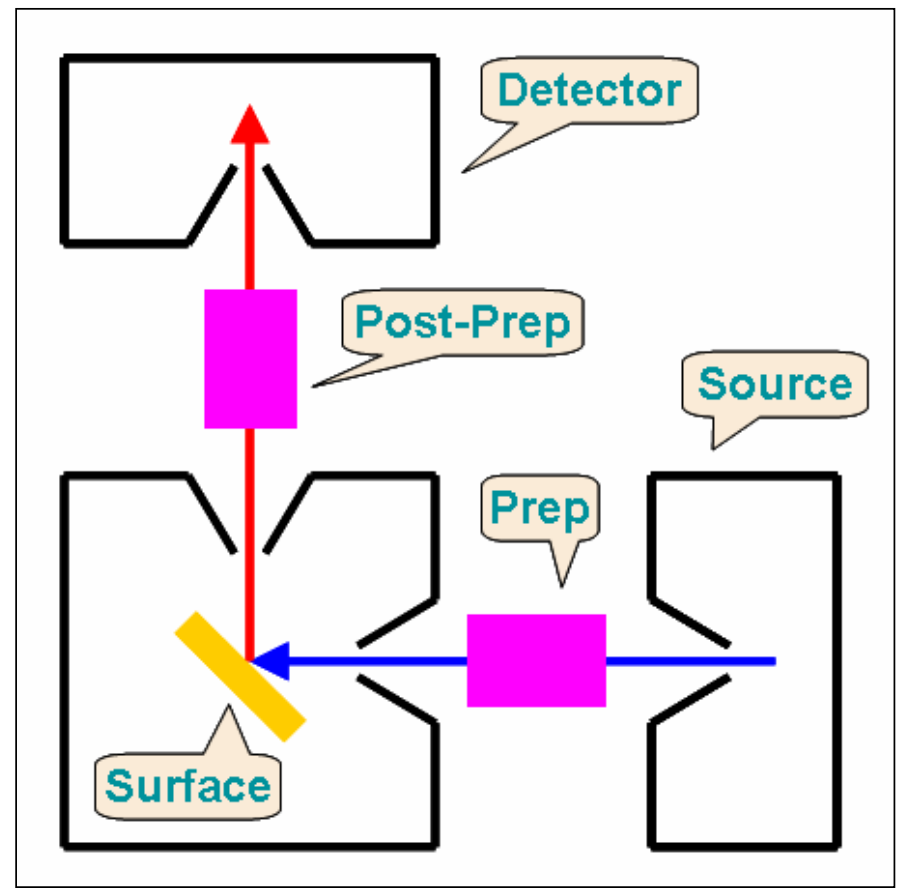

Figure 3.1 A simplified view of a typical scattering experiment. The molecules are introduced into the vacuum in the source. The molecules can be prepared into specific quantum states or velocity selected prior to scattering from the surface. The post prep allows for state specific detection or preparation into other quantum states which can then be detected. ${ }^{25}$

A set-up, such as that shown in Fig. 3.1, is employed in the experimental work in this thesis. The source is used to introduce the molecules into the vacuum chamber, with knowledge over the quantum state of the molecules. The preparation area can be used to alter the molecular beam in various ways, such as optical pumping to higher vibrational/electronic states, velocity selection via mechanical choppers, orientation, and so on. After preparation of the desired quantum states and physical characteristics, the molecules interact with the surface. The surface is often a single metal crystal or other well defined surface. Special care is taken to ensure the surface is as clean and well defined as possible. This usually entails some combination of cleaning techniques, annealing, confirmation of crystalinity, detection of surface impurities etc. After interacting with the surface, scattered molecules can be detected in a variety of ways such as quadrapole mass detection, laser-induced fluorescence (LIF), REMPI, etc. Each of these detection schemes has its own advantages and disadvantages. For studies of vibrational excitation such as those contained 
within this thesis, optical detection methods have been commonly used, REMPI has been shown to be particularly good for detecting both $\mathrm{NO}$ and $\mathrm{HCl}$, the molecules used in this thesis. From this broad simplified outline, we will now turn to the specific details of the work carried out here.

\subsection{Ultrahigh Vacuum:}

Vacuum has been a key component to fundamental physical chemistry studies for many years. Of the many advantages of vacuum too numerous to list here (increased resistance to arcing, reduction of heat transfer, increased transmission of electromagnetic radiation, etc., etc.) I will focus on only two; the increase in the mean free path and the increase in time for surface adsorbate formation. The explanation for these can be found in any text on kinetic theory of gasses. Table 3.1 shows calculated values of number density, mean free path, surface collision frequency, and time (assuming unit sticking probability) for monolayer formation. From these values it becomes quite clear both the advantages of vacuum and what vacuum is required to undertake the desired experiments.

Table 3.1 A table adapted from Ref. 46 for air at 20 C.

\begin{tabular}{|c|c|c|c|c|c|}
\hline & $\begin{array}{c}\text { Pressure } \\
(\text { Torr })\end{array}$ & $\begin{array}{c}\text { Number } \\
\text { Density } \\
\left(\mathrm{cm}^{-3}\right)\end{array}$ & $\begin{array}{c}\text { Mean Free } \\
\text { Path } \\
(\mathrm{cm})\end{array}$ & $\begin{array}{c}\text { Surface } \\
\text { Collision } \\
\text { Frequency } \\
\left(\mathrm{cm}^{-2} \mathrm{~s}^{-1}\right)\end{array}$ & $\begin{array}{c}\text { Times for } \\
\text { Monolayer } \\
\text { Formation } \\
(\mathrm{s})\end{array}$ \\
\hline Atmosphere & 760 & $2.7 \times 10^{19}$ & $7 \times 10^{-6}$ & $3 \times 10^{23}$ & $3.3 \times 10^{-9}$ \\
\hline Rough Vacuum & $10^{-3}$ & $3.5 \times 10^{13}$ & 5 & $4 \times 10^{17}$ & $2.5 \times 10^{-3}$ \\
\hline High Vacuum & $10^{-6}$ & $3.5 \times 10^{10}$ & $5 \times 10^{3}$ & $4 \times 10^{14}$ & 2.5 \\
\hline Very-High Vacuum & $10^{-9}$ & $3.5 \times 10^{7}$ & $5 \times 10^{6}$ & $4 \times 10^{11}$ & $2.5 \times 10^{3}$ \\
\hline Ultra-High Vacuum & $<10^{-10}$ & $3.5 \times 10^{4}$ & $5 \times 10^{9}$ & $4 \times 10^{8}$ & $2.5 \times 10^{6}$ \\
\hline
\end{tabular}

For my experiments it is important that the molecules are unlikely to undergo a collision either before or after interacting with the surface. These conditions ensure that any change in the energy or other characteristics of the molecule arise only due to interactions with the surface. In my experiments, and molecular beam experiments as a rule, much effort is made to prepare the molecules in specific well known quantum states with a known velocity prior to the scattering event. Any perturbation to the gas molecules would cause changes in both the velocity and quantum state distribution of the beam. From Table 3.1 it can be seen that a base pressure of $10^{-6}$ Torr will give a mean free path much longer than the flight path used in this work. The other important consideration for vacuum mentioned earlier is cleanliness of the surface. At $10^{-6}$ Torr a monolayer of adsorbates forms on the order of a few seconds. This is clearly not enough time to complete a scattering experiment on a clean well defined surface. A better vacuum is required in order to increase the time for monolayer formation. To obtain higher vacuum without compromising the intensity of the molecular beam my machine uses two stages of differential 
pumping between the source and the surface chamber. Utilizing differential pumping allows for a large gas load to be produced in the source chamber, while maintaining a low pressure downstream in the molecular beam. With the beam running the machine has a pressure of $\sim 10^{-5}$ Torr in the source chamber and $\sim 10^{-9}$ Torr in the surface chamber allowing enough time for experimental measurements. In the machine used in this thesis the differential regions are as short as possible, $55 \mathrm{~mm}$ and $25 \mathrm{~mm}$ for differential 1 and 2, respectively. This short distance gives a more intense molecular beam at the surface.

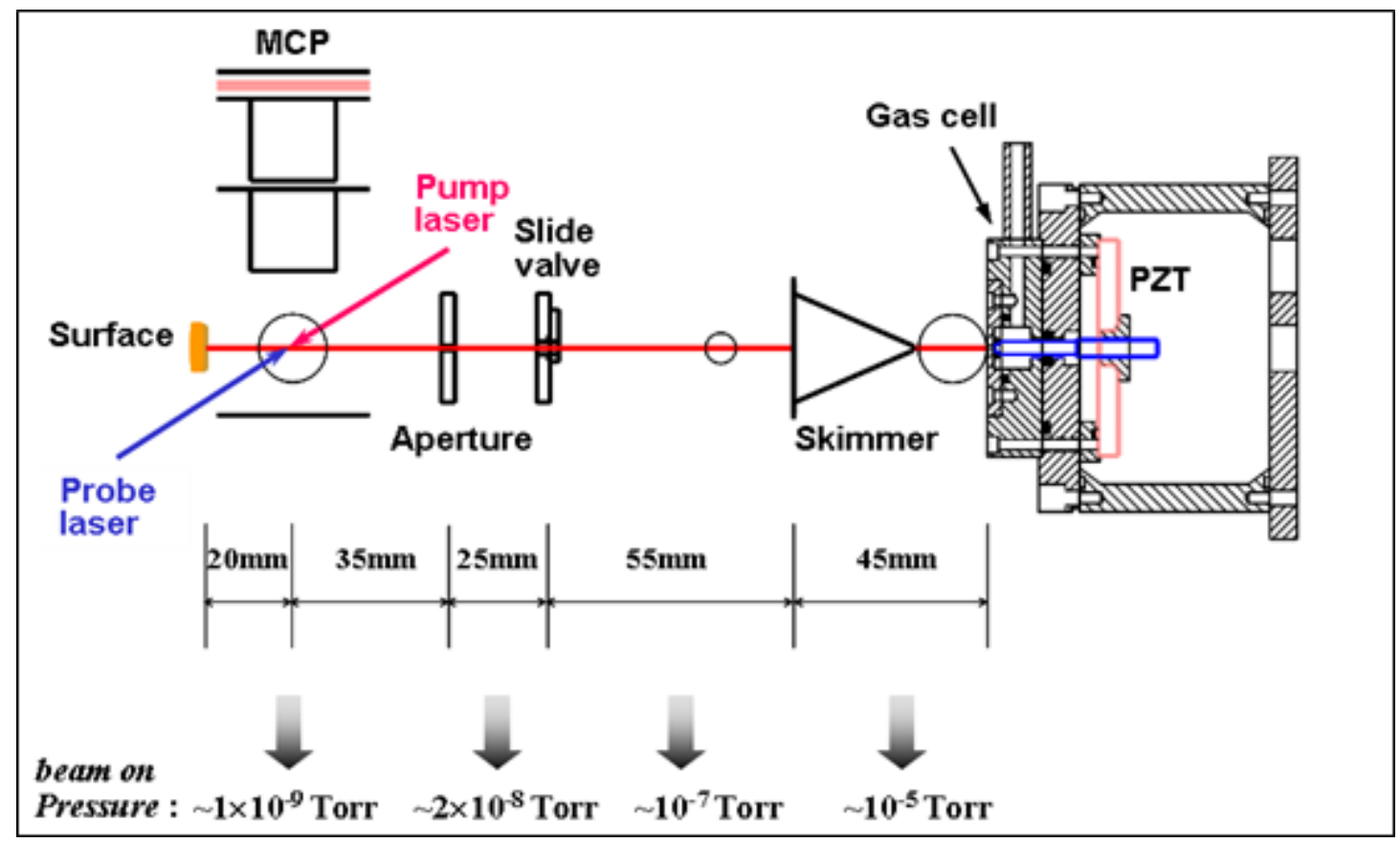

Figure $3.2 \mathrm{~A}$ schematic of the molecular beam machine. The pulsed valve is shown on the right. The beam exits the valve and passes through the skimmer. The beam passes through a slide valve and an aperture before entering the surface chamber. Optical ports allow both UV and IR light to enter for pumping and probing of the molecules both before and after scattering from the surface. The ionized molecules are detected by the MCP. The lengths and pressures of the four differentially pumped chambers are shown. ${ }^{47}$

\subsection{Molecular Beams:}

Molecular beams have a long history in both surface and gas phase chemistry, for good reason. Molecular beams have three important attributes utilized to carry out this work. First, combined with sufficient vacuum the molecular beam is produced in a collision free environment, ensuring the molecules are free from any perturbing influences. Second, the molecular beam can be produced in a way that carefully controls the propagation of the beam in terms of velocity, size, duration, etc. Third, molecular beams can be created in a way that molecules in the beam are populated in only a few quantum states, simplifying greatly the number of initial conditions one must consider in the experiment. Molecular beams can be both effusive and supersonic. In this work, I have used pulsed supersonic beams. Compared to effusive beams, supersonic beams have the advantage of a narrower velocity spread and internal quantum state distribution. Compared to a continuous source, pulsed sources can produce a much more intense beam, as well as 
introducing less gas load into the source chamber. Due to the large difference between the backing pressure in the pulsed valve and the background pressure of the vacuum into which it expands the Mach disk which is caused by beam background interactions is much further from the nozzle than in continuous sources. This means that pulsed sources also minimize shockwaves. $^{48}$

My valve is based on a design by Proch and Trickl,${ }^{49}$ with important modifications. It is piezoelectrically driven; a drawing of the pulsed valve is in Fig. 3.3.

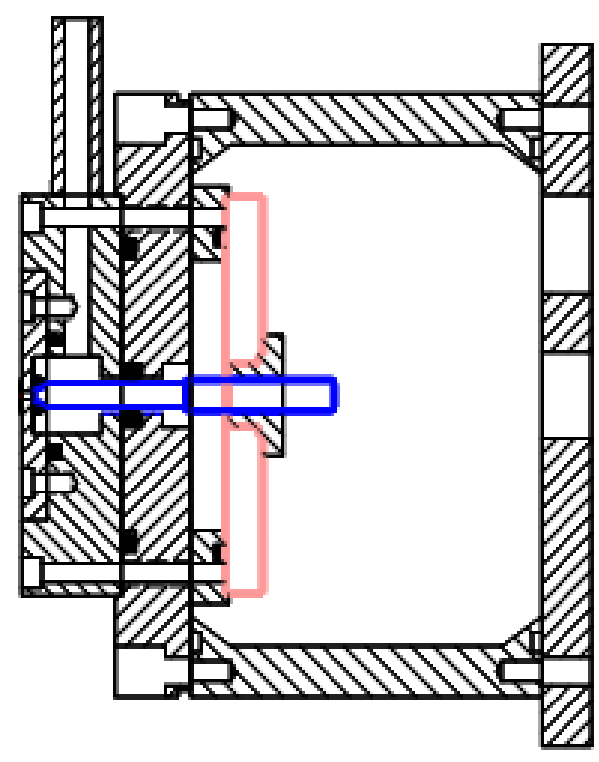

Figure 3.3 Drawing for the pulsed valve. The plunger is outlined in blue, the piezocrystal in red. When voltage is applied across the piezocrystal it contracts moving the plunger away from the faceplate. This causes the O-ring on the tip of the plunger to lose its seal and gas expands from the nozzle. An O-ring halfway up the plunger isolates the piezocrystal from the corrosive gases used in this study. ${ }^{47}$

The gas molecules are released from a stagnation pressure of $3 \mathrm{~atm}$, through a $1 \mathrm{~mm}$ opening on the face of the valve. An O-ring on the tip of the plunger, blue, seals the orifice of the valve. The plunger is driven by the piezo-crystal, red, (Physik Instumente $100 \mu \mathrm{m}$ travel). Applying high voltage to the crystal causes it, and consequently the plunger, to contract away from the faceplate. This in turn causes the O-ring on the tip of the plunger to break its seal releasing gas into the chamber. One important feature of the valve is the dynamically sealing O-ring half-way up the plunger. This addition has the advantage of isolating the piezo-crystal from the gas. For the work involving $\mathrm{HCl}$ this was a critical factor in completing the experiments due to the short lifetime of the piezo-crystal when exposed to $\mathrm{HCl}$. For production of molecular beams of $\mathrm{NO}$ this dynamically sealing O-ring was removed.

One common tool in using molecular beams is seeding. That is, a small fraction of the molecule of interest is seeded into inert gases of varying masses. In expansions lighter gases expand out of 
the valve with a higher velocity, however when a mixture is made, the velocity of the beam is a function of both of masses in the mixture and their relative concentrations. Ref. 48 contains a more detailed explanation. For a very dilute beam $\sim 1 \%$ of $\mathrm{NO}$ in $\mathrm{H}_{2}$ the velocity of the beam $(\sim 2700 \mathrm{~m} / \mathrm{s})$ is only a bit slower than that of a pure $\mathrm{H}_{2}$ beam $(\sim 2900 \mathrm{~m} / \mathrm{s})$. By adjusting the carrier gas and the concentration, velocities from 400 to $2700 \mathrm{~m} / \mathrm{s}$ can be achieved. The spread in velocities in molecular beams is typically quite low. For example, the $\Delta \mathrm{v} / \mathrm{v}$ for different beams of $\mathrm{HCl}$ ranging in speed from $1200 \mathrm{~m} / \mathrm{s}$ to $2600 \mathrm{~m} / \mathrm{s}$ is in the range of $0.06-0.07 .{ }^{50}$

\subsection{Molecular Spectroscopy:}

Molecular spectroscopy involves interaction of electromagnetic radiation with atoms or molecules. There are a great number of texts on the subject, ${ }^{51-55}$ so only a brief overview will be given. Much of what we know about the structure of atoms and molecules comes from spectroscopic investigations. Wavelength measurements of spectral lines allows for the determination of the energy levels in an atomic or molecular system. The intensity of these spectral features is proportional to the transition probability, which is a measure of how strongly two energy levels are coupled, as well as the population difference of the two states. This transition probability depends on the wave functions of both levels, which can be calculated from solutions, albeit approximately, of the Schrodinger equation. The intensity measurements can be used to verify these calculations. Excited state lifetimes can be calculated by the width of the spectral line or by using time resolved (for example fluorescence) methods. Measurements of the Doppler width allow for the determination of the temperature of the sample. Pressure broadening and pressure shifts yield information on the collision processes and intermolecular forces. Zeeman and Stark splittings, by external fields allow for the measurement of coupling between the different angular momentum in atoms and molecules. The hyperfine structure present in the spectral lines provides the magnetic and electric moments and allows dipole or quadrupole moments to be calculated. Time resolved measurements allow dynamical process in both the ground and excited states to be followed in "real time".

With this wide range of possibilities, it is clear why spectroscopy has been for some time a workhorse in practically all fields of the natural sciences. Despite the seemingly endless possibilities for spectroscopy, the quality of the spectra is limited by the spectral and time resolution of the various techniques employed. Advances in optical instrumentation and development of new techniques increased the spectral and time resolution. However, the single biggest advance for spectroscopy was the advent of the laser. The laser allowed for several orders of magnitude improvement in both spectral and time resolution, in comparison to incoherent light sources. Lasers allowed experiments that were not possible with non-coherent light to be undertaken, and new fields of spectroscopy grew only after the advent of the laser.

In this work I employ resonant enhanced multiphoton ionization (REMPI) to determine the populations of individual quantum states of the molecules, both before and after interacting with the surface. REMPI utilizes resonant pumping from, in this case, the ground electronic state to an 
excited electronic state. Once excited, the molecule adsorbs an additional photon and is excited to the ionization continuum, producing an ion that is then detected. One and two color REMPI schemes are commonly used. In this work one color $(2+1)$, for $\mathrm{HCl}$, and $(1+1)$, for NO, REMPI schemes are utilized. The numbers in the REMPI scheme correspond to the number of photons required for the resonant and the ionization step respectively.

\subsection{Optical pumping:}

In addition to spectroscopic detection, lasers can also be used to prepare molecules in specific quantum states. As explained above, molecules when in resonance with a specific photon energy can absorb or emit a photon. One side effect of probing molecules is that the molecules are in a different quantum state after being probed. Taking advantage of this I can create molecules in excited electronic, vibrational, and rotational states. In fact, now it is not uncommon to utilize two lasers in preparation of molecules such as in pump-dump experiments. ${ }^{9,10,56}$ In this work I have prepared vibrationally excited molecules by IR vibrational overtone pumping of NO $(\mathrm{v}=3 \leftarrow 0)$ and $\mathrm{HCl}(\mathrm{v}=2 \leftarrow 0)$. Vibrational overtone pumping is forbidden by optical selection rules in a harmonic oscillator, however anharmonicity in the oscillator allows for overtone excitations. The strength of these transitions depends on the strength of the anharmonicity, but are much less than the allowed $\Delta v=1$ transitions, for example the NO first overtone is 65 times weaker than the fundamental vibrational excitation ${ }^{57}$. Despite the weakness of the overtone transition sufficient laser intensities can create populations of vibrationally excited molecules in amounts sufficient $\left(\mathrm{P}_{\text {overtone }} \sim 10^{-3}\right)$ for this study. The overtone pumping efficiency was estimated by comparing the REMPI intensity of vibrationally overtone pumped molecules to that of ground state. Experimental details will be presented in following chapters.

\subsection{Ion Optics:}

The ion detection apparatus used in these experiments is homebuilt. It detects the ions' mass by time of flight (TOF). The system consists of a repeller, two cylindrical electrostatic optics, and a micro channel plate (MCP) detector and is shown in Fig. 3.4. 


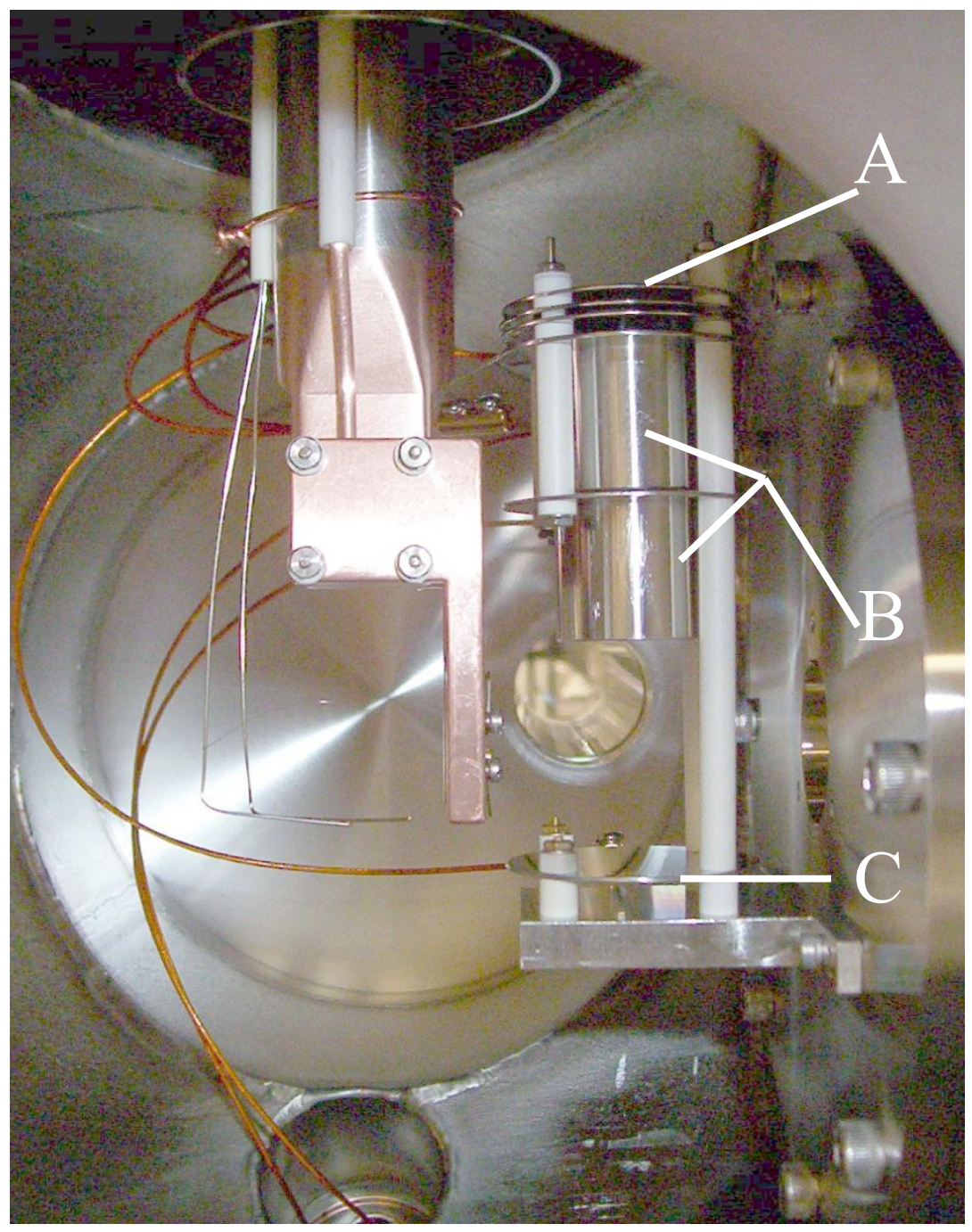

Figure 3.4 Ion collection apparatus. The molecular beam enters from the right. The surface is attached to the copper arms, and is just barely visible. A. MCP, B. electrostatic lenses, C. Repeller. ${ }^{47}$

The repeller and optics focus the ions onto the MCPs. The MCPs are in a chevron configuration, where two plates are oriented such that the angle of the channels induces a directional change in the electrons. ${ }^{58}$ The resulting current was digitized on a scope (Wavesurfer 452), which is controlled via USB with a computer. All the software for reading the signal was designed in house and written in LabVIEW ${ }^{\mathrm{TM}}$.

The ion flight distance of $\sim 6 \mathrm{~cm}$ provides enough mass resolution to separate $\mathrm{Cl}^{-}$isotopes. Special care was taken to adjust the voltages so that the collection efficiency remained constant over a large volume where the laser crosses the plume of scattered molecules. This was checked by filling the chamber with background $\mathrm{HCl}, \sim 5 \times 10^{-6}$ Torr, and moving the REMPI laser both perpendicular and parallel to the incident molecular beam. 


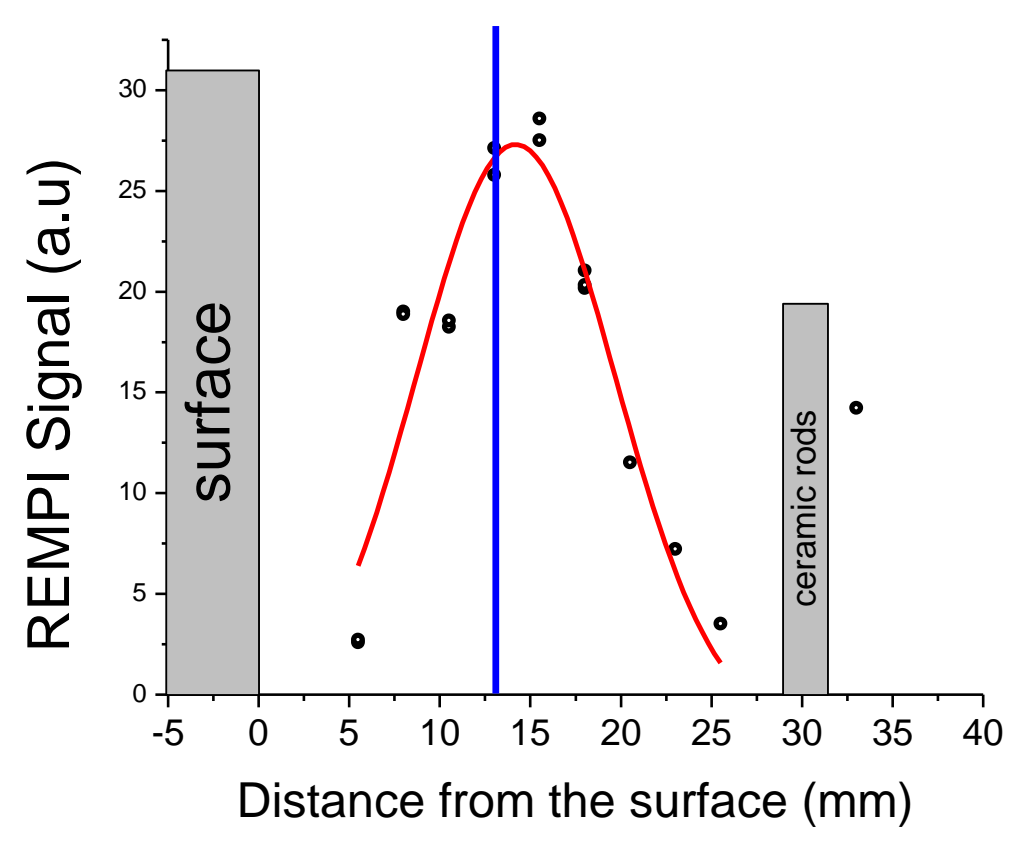

Figure 3.5 Dependence of the ion signal as a function of the distance from the gold surface parallel to the molecular beam. The red line is a Gaussian fit to the data. The blue line is the approximate position of the REMPI laser for all experiments made later.

The location of the ionization laser is very important, especially in the search for weak signals, as the collection efficiency of the ions depends on the location of the ionization laser along the molecular beam, as shown in Fig. 3.5. I chose a location near the peak of the collection efficiency where I was also able to collect accurate angular distributions.

Angular distributions are important for determining direct scattering, and are used in the determination of excitation or survival probabilities. Therefore, it is critical to determine if the ion collection efficiency is affected when translating the probe laser beam perpendicular to the molecular beam. 


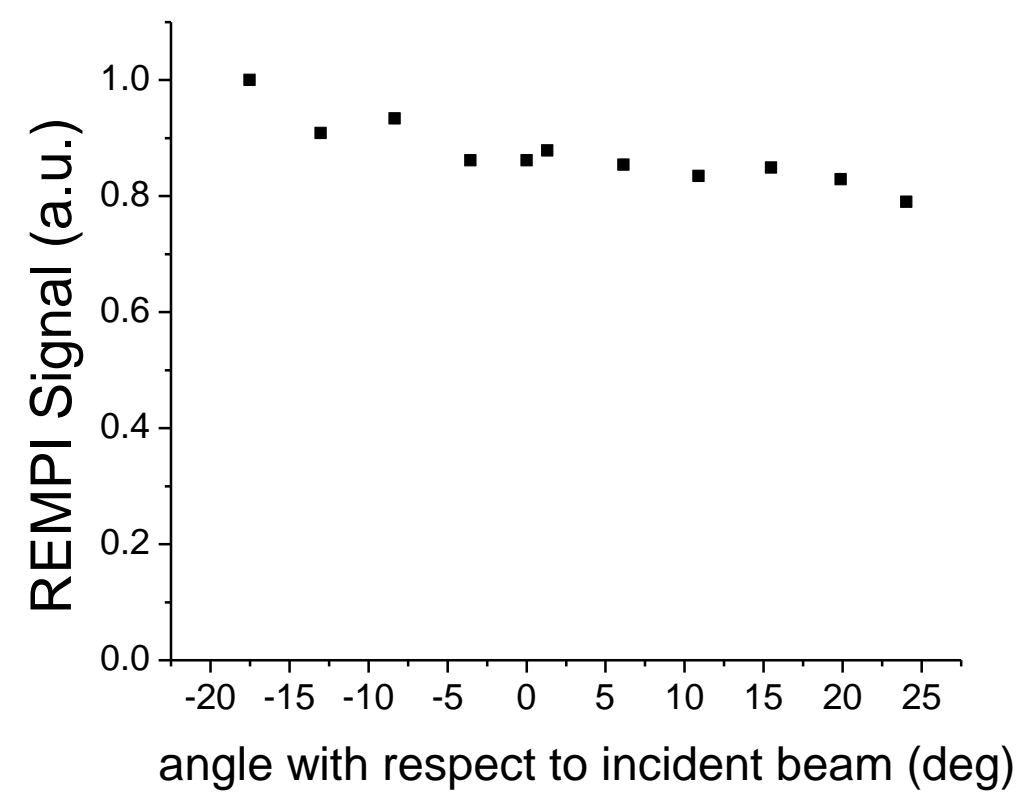

Figure 3.6 Intensity of the REMPI signal of background of $\sim 5 \times 10^{-6}$ Torr as a function of laser position perpendicular to the molecular beam. This angular, relative to the surface normal, dependence on the collection efficiency is taken $13 \mathrm{~mm}$ from the surface corresponding to the blue line in Fig. 3.5.

A relatively constant REMPI signal was obtained as a function of the angle with respect to the incident beam. This allows angular distributions to be measured without correction factors. Additional information on ion collection efficiency is located in Appendix II.

From Fig. 3.5 and 3.6 we can find conditions that meet goals of ion collection: high collection efficiency, consistent ion collection efficiency when translating the beam perpendicular to the molecular beam, and adequate distance from the surface to accurately determine the velocities of the scattered molecules. In all the work shown here, I assume no functional dependence on ion collection efficiency as a function of angle with respect to the incident beam.

\subsection{Pulsing MCPs:}

It was sometimes required to replace the tungsten wires which hold the surface in place. At one point with new wires, inconsistencies with REMPI signal were noted. After some effort it was determined that positive ions emitted from the new tungsten wires were being collected by the ion optics creating a DC signal on the MCP. The problem with a large background DC signal is that the electrons in the MCP cannot be replenished fast enough to produce the proper gain for the ions created by the probe laser. This is especially problematic when looking for small signals as the MCP gain is maximized, which in turn also maximizes the DC signal from the positive ions emitted from the tungsten wires leading to depletion of electrons in the MCP. 


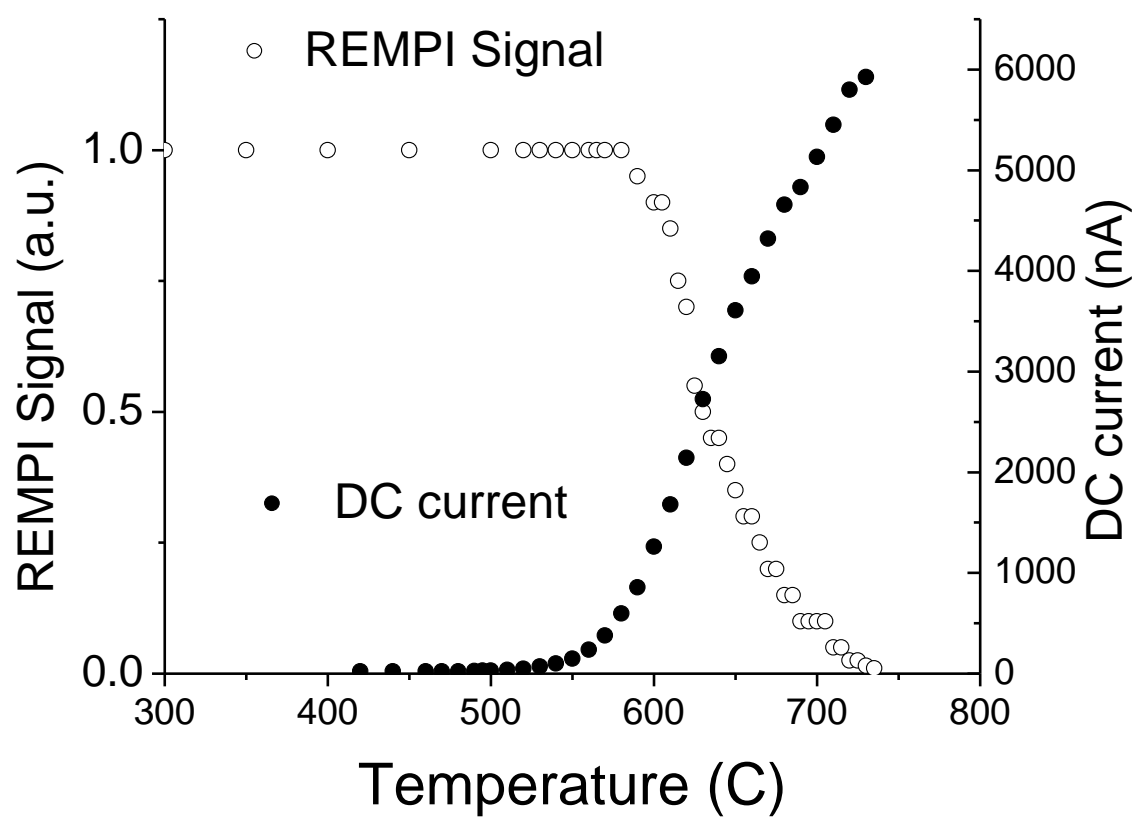

Figure 3.7 REMPI signal of NO in the incident beam, empty circles, and DC current, filled circles, on the MCP as a function of surface temperature at an MCP voltage of $1900 \mathrm{~V}$.

The DC current shown in Fig. 3.7 increases dramatically ( 300 fold increase from 400-700 C) when the voltage across the MCP is $1900 \mathrm{~V}$, near its maximum. A small increase in the DC current seems to have no effect on the MCP gain, until a threshold is crossed and the gain of the MCP drops quite dramatically.

Many attempts were made to mediate this problem: biasing the surface, changing the voltages on the repller/lenses, none of which were satisfactory. I observed that when the voltage on the MCP was set to $1300 \mathrm{~V}$, less than the maximal $(2000 \mathrm{~V})$ value, the DC current was not a significant problem, because the emitted ions striking the MCP produced less DC current and consequently the MCP's electrons were not depleted sufficiently to reduce the gain. With this information, I experimented with pulsing the MCP voltage in the hope that this would minimize the electron depletion. Pulsing of the MCP was achieved by the utilization a fast switch solid state pulser (DEI PVX-4140). There are several adjustable parameters of the pulsing conditions: the duration of the pulse, the timing of the pulse relative to the firing of the REMPI laser, the base voltage of the pulse, etc. After many experiments, I noticed that the length of the pulse didn't seem to matter as long as it was small compared to the pulse frequency $(10 \mathrm{~Hz})$ and the timing of the pulse relative to the firing of the REMPI laser was the most important factor. It is imperative to start the voltage pulse on the MCP as close to the firing of the REMPI laser as possible. Scattered light from the REMPI laser can be seen on the MCP, this is used as the reference. I found that starting the high voltage pulse $200 \mathrm{~ns}$ before the firing of the REMPI laser allowed for a strong REMPI ion signal and no problems with electron depletion from stray ions. 


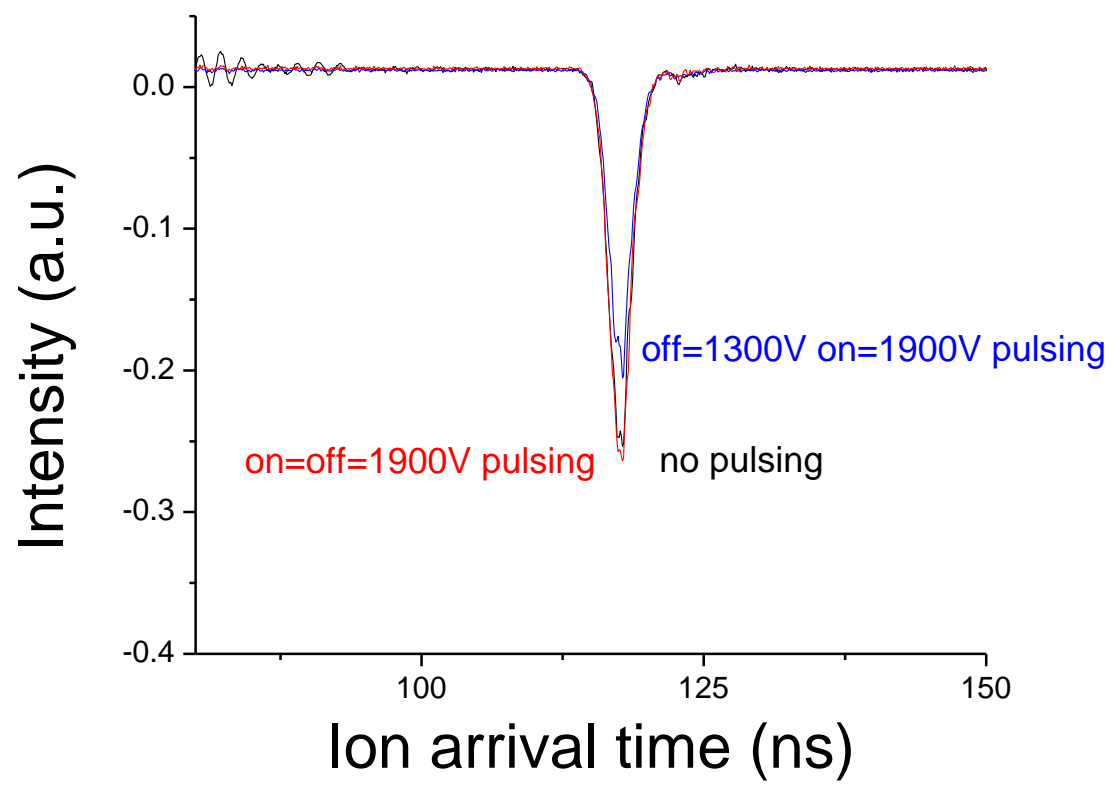

Figure 3.8 Raw REMPI signal, for different MCP pulsing schemes, when the surface is cold and DC current on the MCP is not a problem: without pulsing $1900 \mathrm{~V}$, black; with pulsing from $1900 \mathrm{~V}$ to $1900 \mathrm{~V}$, red, and pulsing from 1300-1900 V, blue.

One of the important aspects of pulsing the MCPs is to ensure that pulsing does not degrade the ability to detect ions. To check this, the incident beam was probed and the ions detected in three pulsing schemes with a room temperature surface when depletion of the MCPs is not problematic. These three schemes are shown in Fig. 3.8, black with no pulsing $\mathrm{MCP}=1900 \mathrm{~V}$, red with the pulsing from $1900 \mathrm{~V}$ to $1900 \mathrm{~V}$ and blue with pulsing from $1300 \mathrm{~V}$ to $1900 \mathrm{~V}$. Here I see that the difference between the three pulsing schemes is very small and that pulsing the MCPs does not negatively affect the MCP gain. Verification of the success of pulsing the MCPs comes also from seeing an effect of pulsing when the surface is hot and the MCPs are electronically depleted. 


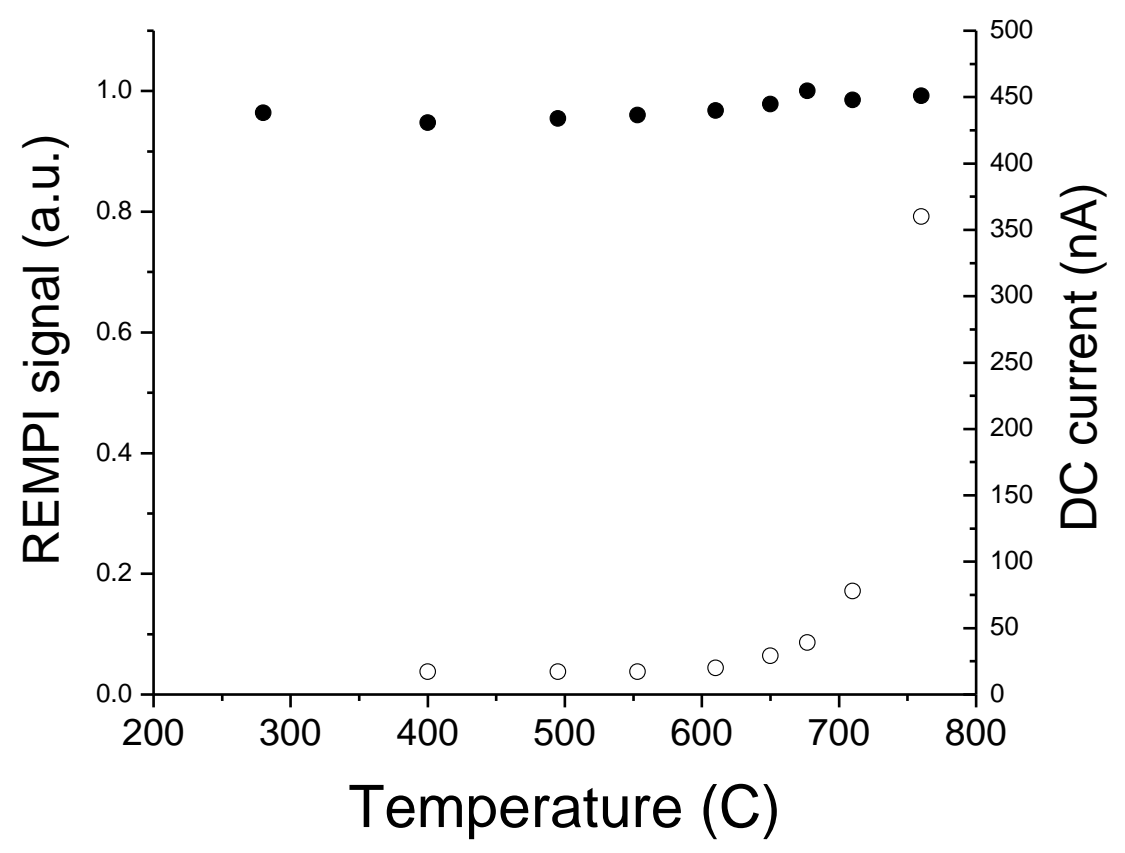

Figure 3.9 Similar to Fig. 3.7; DC current of the MCP, empty circles, and REMPI signal ( $\mathrm{v}=0 \mathrm{~J}=1.5)$ of incident beam, filled circles, as a function of surface temperature, while pulsing the MCP from $1300 \mathrm{~V}$ to $1900 \mathrm{~V}$.

In Fig. 3.9 the REMPI signal is almost completely independent of the surface temperature. The DC current, although increasing has not yet crossed the threshold value for electron depletion, which is empirically found to be near $500 \mathrm{nA}$. In summary, pulsing of the MCP resulted in no degradation of the signal and circumvented the electron depletion of the MCP caused by ions emitted from the hot tungsten wire. Another possible solution to this problem is using tantalum wires instead of tungsten. Tantalum has a much lower concentration of potassium, the likely ions emitted from the hot tungsten wires, and would likely be less problematic. I purchased Tantalum wire (Alfa Aesar, $0.25 \mathrm{~mm} \mathrm{99.9+ \%} \mathrm{pure),} \mathrm{although} \mathrm{it} \mathrm{has} \mathrm{not} \mathrm{yet} \mathrm{been} \mathrm{experimentally} \mathrm{tested.}$

\subsection{Surface Preparation:}

The metal sample used in these studies is a $\mathrm{Au}(111)$ crystal (Monocrystal $10 \mathrm{~mm}$ diameter $2 \mathrm{~mm}$ thick). The sample is attached to a homebuilt sample holder at the end of a $\sim 1.45 \mathrm{~m}$ cold finger, that can be filled with liquid nitrogen. The cold finger is attached to a high precision XYZ $\Theta$ manipulator capable of $800 \mathrm{~mm}$ translation along the vertical $\mathrm{Z}$ axis, $50 \mathrm{~mm} \mathrm{X}$ and $\mathrm{Y}$ translations in the horizontal plane and $360^{\circ}$ rotation around $\mathrm{Z}$ axis. These motions allow for precise placement for a multitude of tasks, such as ion bombardment cleaning, annealing, Auger spectroscopy, beam scattering, etc. The sample is attached to the copper arms of the sample holder by tungsten wires. These wires provide the resistance for the heating of the sample as well as conductive cooling from the cold finger. A K-type, 0.003", thermocouple is inserted into a small hole drilled into the side of the crystal. For the NO experiments a second K-type thermocouple was attached, For all surface temperatures there was no difference between the two thermocouples. Between 
the copper arms and the cold head sapphire spacers are used to allow thermal conductivity, while electronically insulating the fingers. The copper fingers are attached to electrical feedthroughs via copper rods, where a DC power supply (Electronic Measurement, EMS7.5-130) passes the current that heats the crystal.

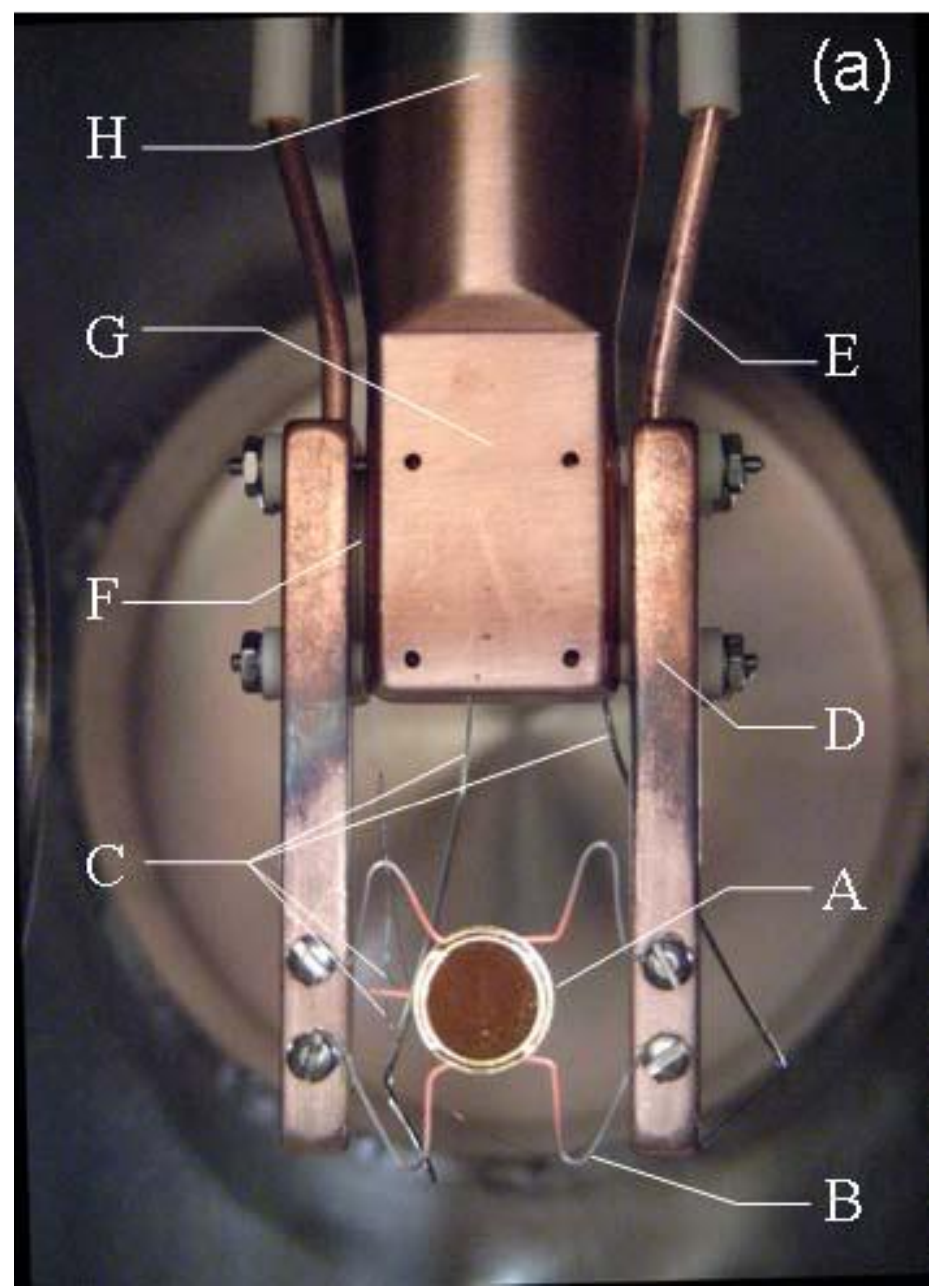

Figure 3.10 Picture of the sample holder, A. $10 \mathrm{~mm}$ diameter $\mathrm{Au}(111)$ crystal (hot); B. tungsten wires; C. thermocouple wires (K-type); D. copper arms; E. electrical connection; F. sapphire spacer; G. copper cold finger; H. stainless steel cryo-tube, not shown, a second thermocouple attached in the same manner as $\mathrm{C}$ used in NO vibrational excitation measurements. ${ }^{47}$

The sample is cleaned with a combination of $\mathrm{Ar}^{+}$ion sputtering and annealing. As seen in Fig. 3.11 the main impurity on the surface is carbon, but this can be easily removed with $\mathrm{Ar}^{+}$ion sputtering. 


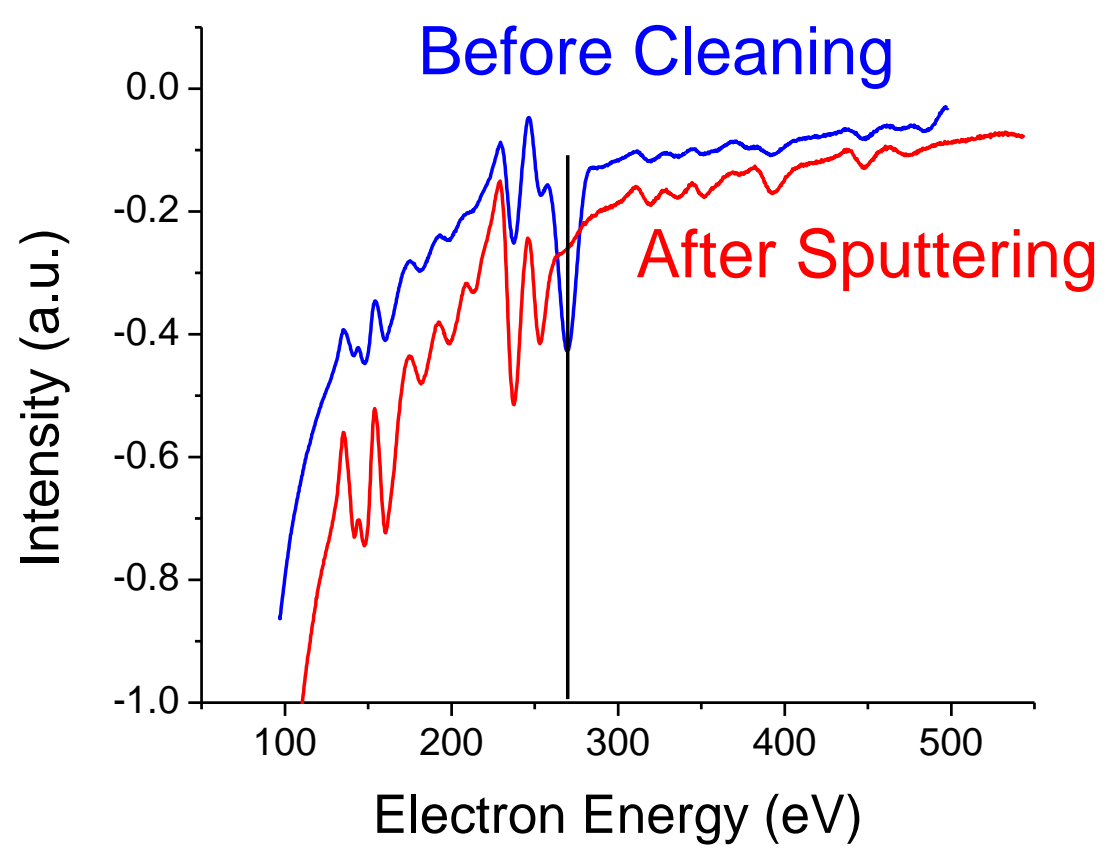

Figure 3.11 Typical auger spectra before, blue, and after, red, $\mathrm{Ar}^{+}$ion sputtering, scaled arbitrarily. The black vertical line represents the location of the carbon peak. The other peaks are gold peaks.

The surface was sputtered for $\sim 60$ minutes by $\mathrm{Ar}^{+}$bombardment before every experimental measurement. Auger spectra were taken before and after sputtering to verify cleanliness of the surface. After sputtering the impurities in the surface were below the detection limit for the Auger spectrometer, 0.03 monolayer. The primary impurities seen were carbon and oxygen. After confirming the cleanliness of the sample, the (111) surface was reconstructed by annealing for $\sim 45$ minutes at $\sim 1000 \mathrm{~K}^{59}$

\subsection{Experimental details for $\mathrm{HCl}$ translational energy transfer:}

The overall aim of this experiment was to look at the translational energy change of $\mathrm{HCl}$ molecules when scattered from a $\mathrm{Au}(111)$ surface. $\mathrm{HCl}$ beams were created by expanding $3 \mathrm{Atm}$ $\mathrm{HCl}$ seeded in carrier gas through a piezo-electrically actuated valve. $\mathrm{HCl}$ beams were created with seven different incidence energies $\left(\mathrm{E}_{\mathrm{i}}=0.28-1.27 \mathrm{eV}\right)$ by varying the concentration of $\mathrm{HCl}$ (1-10\%) in different carrier gases $\left(\mathrm{H}_{2}, \mathrm{D}_{2}, \mathrm{~N}_{2}\right)$. These molecular beams were skimmed and then passed through two stages of differential pumping before entering the scattering chamber. The molecules collided with a $\mathrm{Au}(111)$ crystal at an incidence angle of $3^{\circ}$ with respect to surface normal. The temperature of the crystal can be varied by conductive cooling or resistive heating. The temperature is monitored by K-type thermocouple. I am able to vary the temperature of the surface from $77 \mathrm{~K}$ until the melting point of the crystal $(\sim 1300 \mathrm{~K})$ or higher. For the temperature dependent measurements, the highest temperature used was $1000 \mathrm{~K}$. The surface was sputtered and annealed as explained in section 3.7. 
IR light, $\lambda \sim 1.7 \mu \mathrm{m}$, utilized for vibrational overtone excitation, $\mathrm{HCl}(\mathrm{v}=0 \rightarrow 2)$ was produced by difference frequency generation in a beta barium borate (BBO) crystal. The residual fundamental of a Nd:YAG (yttrium aluminum garnet) laser (Continuum 7010IS) was mixed with the output of a tunable dye laser (Sirah Cobra-Stretch $\left.{ }^{\circledR}\right)$.

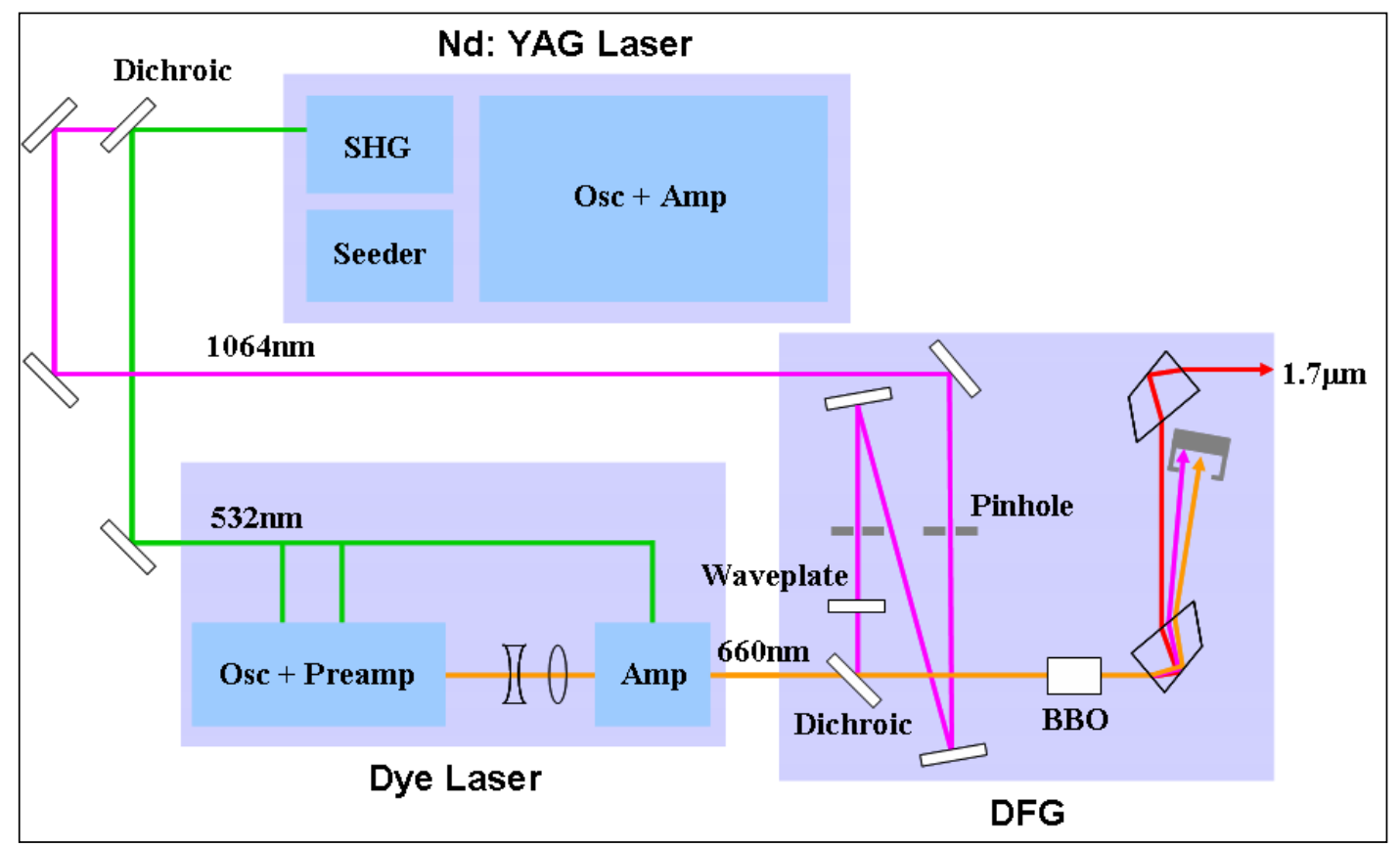

Figure 3.12 Schematic of the IR laser system used for the first overtone pumping of $\mathrm{HCl}$. The second harmonic of a Nd:YAG pumps a tunable dye laser. The output of the dye laser is mixed with the residual fundamental of the Nd:YAG in a BBO crystal. The wavelengths are separated with a Pellin-Broca prism. ${ }^{47}$

The dye laser operated with a mixture of DCM and LDS 698 dyes, and was pumped by the second harmonic of the Nd:YAG. This mixing produced $\sim 3 \mathrm{~mJ} /$ pulse of tunable IR light with a bandwidth of $\sim 1 \mathrm{~cm}^{-1}$. The Continuum 7010IS Nd:YAG was equipped with an injection seeder which would have narrowed the linewidth of the resulting IR light considerably, however the modal structure of the output from the dye laser when combined with narrow bandwidth fundamental from the Nd:YAG created severe instabilities in the overtone pumping efficiency. 


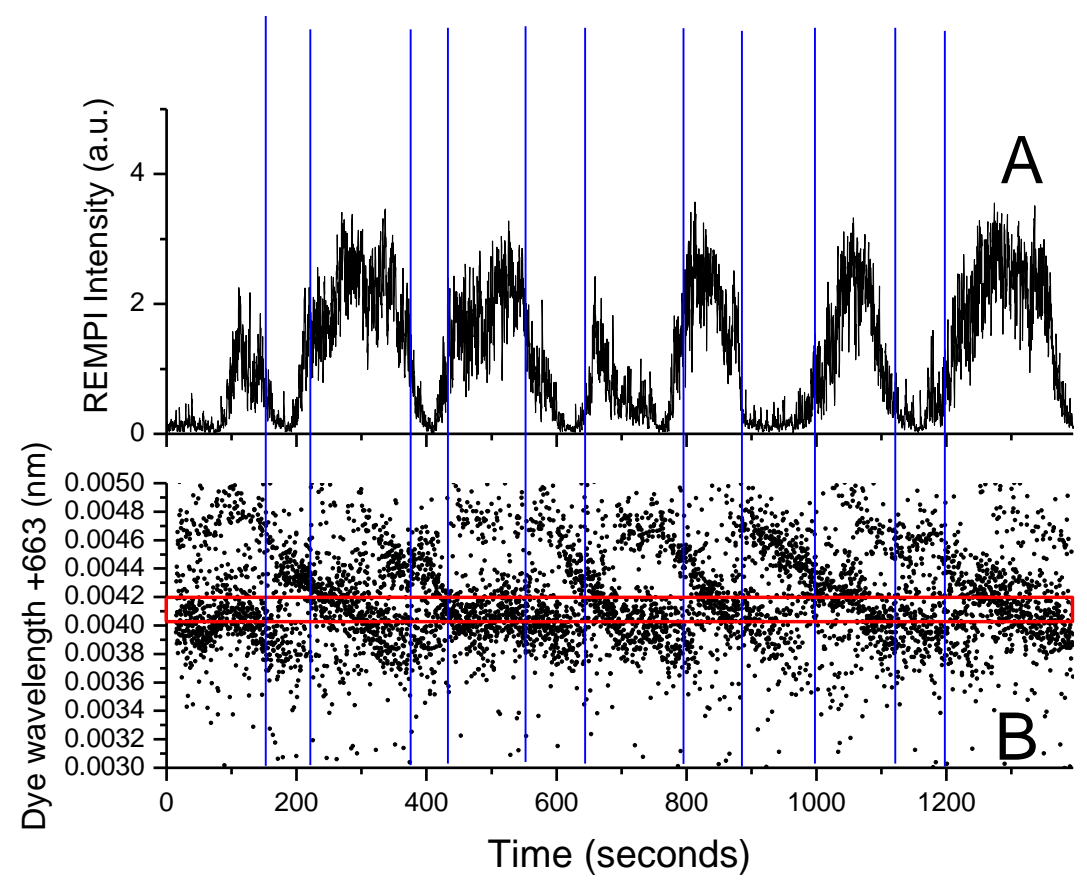

Figure 3.13 Indicates the instability of the vibrational overtone pumping due to mode hopping in the tunable dye laser. Panel A shows the intensity of the REMPI signal of $\mathrm{HCl}(\mathrm{v}=2 \mathrm{~J}=1)$ as a function of time. Panel $\mathrm{B}$ shows the dye laser wavelength of $\sim 663 \mathrm{~nm}$, used to create IR light to pump $\mathrm{HCl}(\mathrm{v}=0 \mathrm{~J}=0 \rightarrow \mathrm{V}=2 \mathrm{~J}=1)$, measured with a wavemeter as a function of time. The red box in the lower panel indicates the wavelength range where the dye output corresponds to overtone vibrational excitation. The vertical blue lines guide the eye. The mode hopping of the dye laser is clear in the panel B.

The mode structure of the output of the dye laser used in IR generation can be seen in Fig. 3.13(B). The red box indicates the wavelength range in which the generated IR light should excite the molecules to $\mathrm{HCl}(\mathrm{v}=2 \mathrm{~J}=1)$. These molecules are then detected via REMPI. The REMPI signal is noticeably absent when the wavelength of the dye laser does not fall into the red box. This indicates that the wavelength of the dye laser leads to the instability in the overtone vibrational pumping of $\mathrm{HCl}(\mathrm{v}=0 \mathrm{~J}=0 \rightarrow \mathrm{v}=2 \mathrm{~J}=1)$.

Turning off the seeder in the Nd:YAG had the effect of lowering the peak pumping efficiency, but made the REMPI signal much more stable. The IR light was focused with a $f=50 \mathrm{~cm}$ lens, creating a waist of $\sim 100 \mu \mathrm{m}$, onto the molecular beam. The overtone excitation efficiency was estimated by comparison of the REMPI intensity of the pumped molecules to that in the ground vibrational state. The excitation efficiency is estimated to be $\sim 10^{-3}$ for $\mathrm{HCl}(\mathrm{v}=0 \rightarrow 2)$.

$\mathrm{HCl}(2+1)$ REMPI was performed using $\mathrm{UV}, \lambda \sim 250 \mathrm{~nm}$ laser pulses produced by doubling the output of a tunable dye laser (Sirah Cobra-Stretch®), operating with Coumarin 503 dye in a BBO crystal. An Inrad Autotracker II is used to match the angle of the BBO crystal with the dye laser wavelength. 


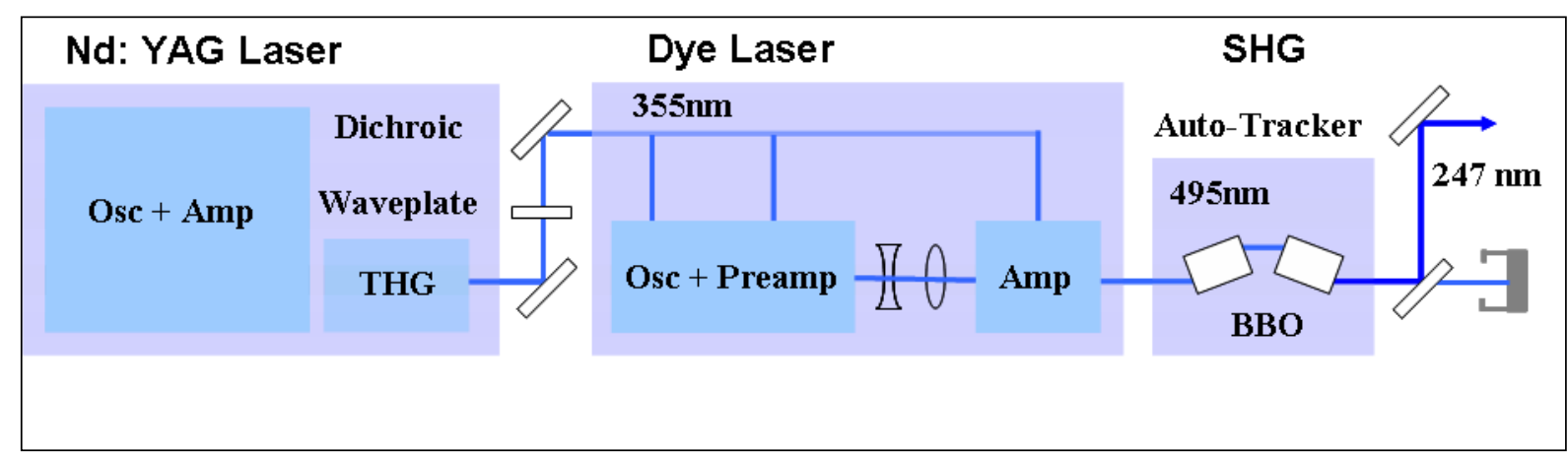

Figure 3.14 Schematic of the UV laser system used for REMPI molecular detection. The third harmonic of a qswitched Nd:YAG $(\sim 355 \mathrm{~nm})$ pumps a tunable dye laser. The output of the dye laser is frequency doubled in a BBO crystal. This allows for UV generation between 220 and $260 \mathrm{~nm}^{25}$

The dye laser was pumped with the third harmonic of Nd:YAG laser (Continuum 7010) and after doubling had an intensity of 4-6 mJ. The UV light was focused into the chamber with a $f=30 \mathrm{~cm}$ lens, creating a waist of $\sim 10 \mu \mathrm{m}$. The focal point of the UV laser was purposely displaced from the center of the molecular beam in order to minimize non-resonant mulitphoton ionization of incident $\mathrm{HCl}$. The resulting ions are collected onto the MCP's as mentioned in section 3.5.

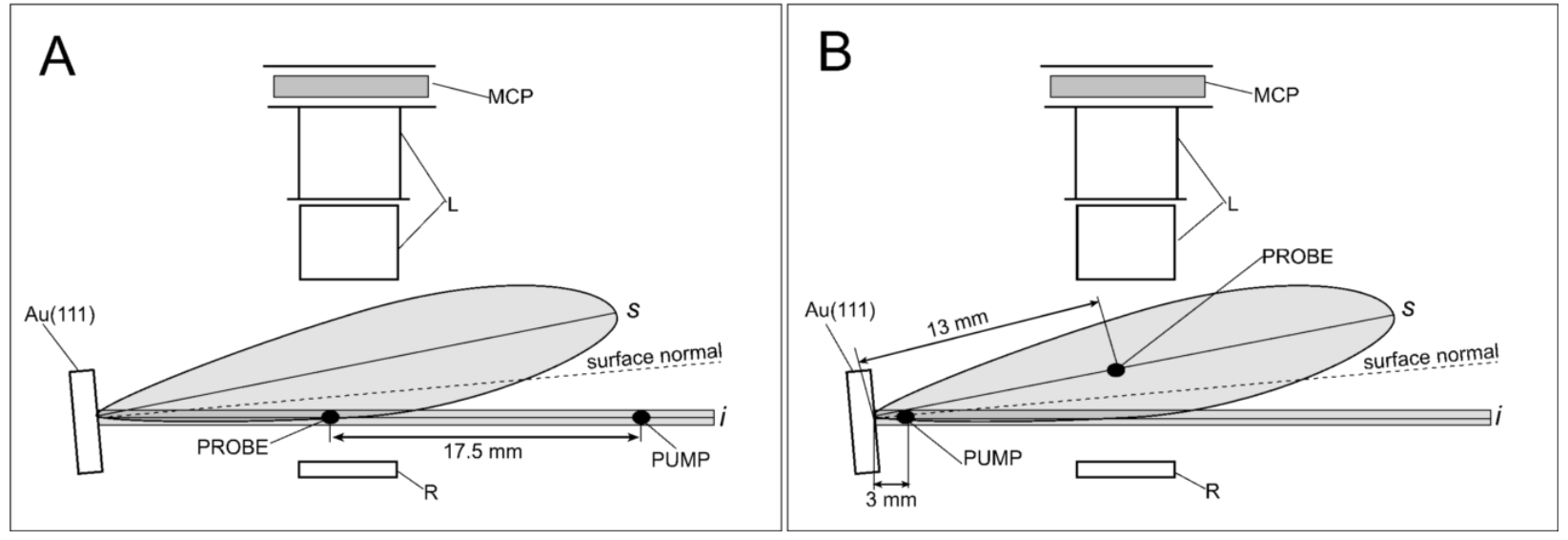

Figure 3.15 The experimental geometry used to measure the kinetic energy of incident (A) and scattered beams (B). The incident beam, $i$, is narrow and well collimated. The scattered beam, $S$, is broad and peaks at the specular angle. The pump beam excites the vibrational overtone and the probe beam ionizes the molecules. The repeller, $\mathrm{R}$, and ion optics, L, guide the ions to the MCP where they are detected. By varying the timing between the firing of the two lasers the TOF of the molecules between the two lasers can be recorded. ${ }^{50}$

To measure the kinetic energy of the incident beam the molecules are pumped from the ground vibrational state $\mathrm{HCl}(\mathrm{v}=0 \mathrm{~J}=0 \rightarrow \mathrm{v}=2 \mathrm{~J}=1)$, using IR overtone excitation. $17.5 \mathrm{~mm}$ downstream the excited molecules are detected via state specific REMPI as seen in Fig. 3.15(A). The velocity distributions of the molecular beam can be derived from the raw time of flight (TOF) data combined with accurate knowledge of the distance between the pump and probe lasers. The conversion from raw arrival time measurements to velocity and subsequently translational energy is shown in Appendix I. 
Measurement of the scattered beam is done in an analogous fashion to the incident beam. The molecules are pumped to $\mathrm{HCl}(\mathrm{v}=2 \mathrm{~J}=1)$ using the first overtone transition. Since the molecular beam spreads out in time due to the inherent velocity spread of the beam, the IR pumping occurs near the surface (within $3 \mathrm{~mm}$ ) to minimize the uncertainty in time at which the excited molecules strike the surface. All results shown here, unless otherwise stated, occurred with the probe laser being placed at the specular angle. Mounting the steering optics for both the pump and probe lasers on micrometric stages, with two dimensions of travel, combined with removable apertures accurately positioned on the optical windows, allows for fast and accurate switching between the two geometries shown in Fig. 3.14 and any others that may be desired. The translational stages used for the movement of the IR pump beam are shown in Fig. 3.16. A similar translational stage is used to quickly align the REMPI laser as well.

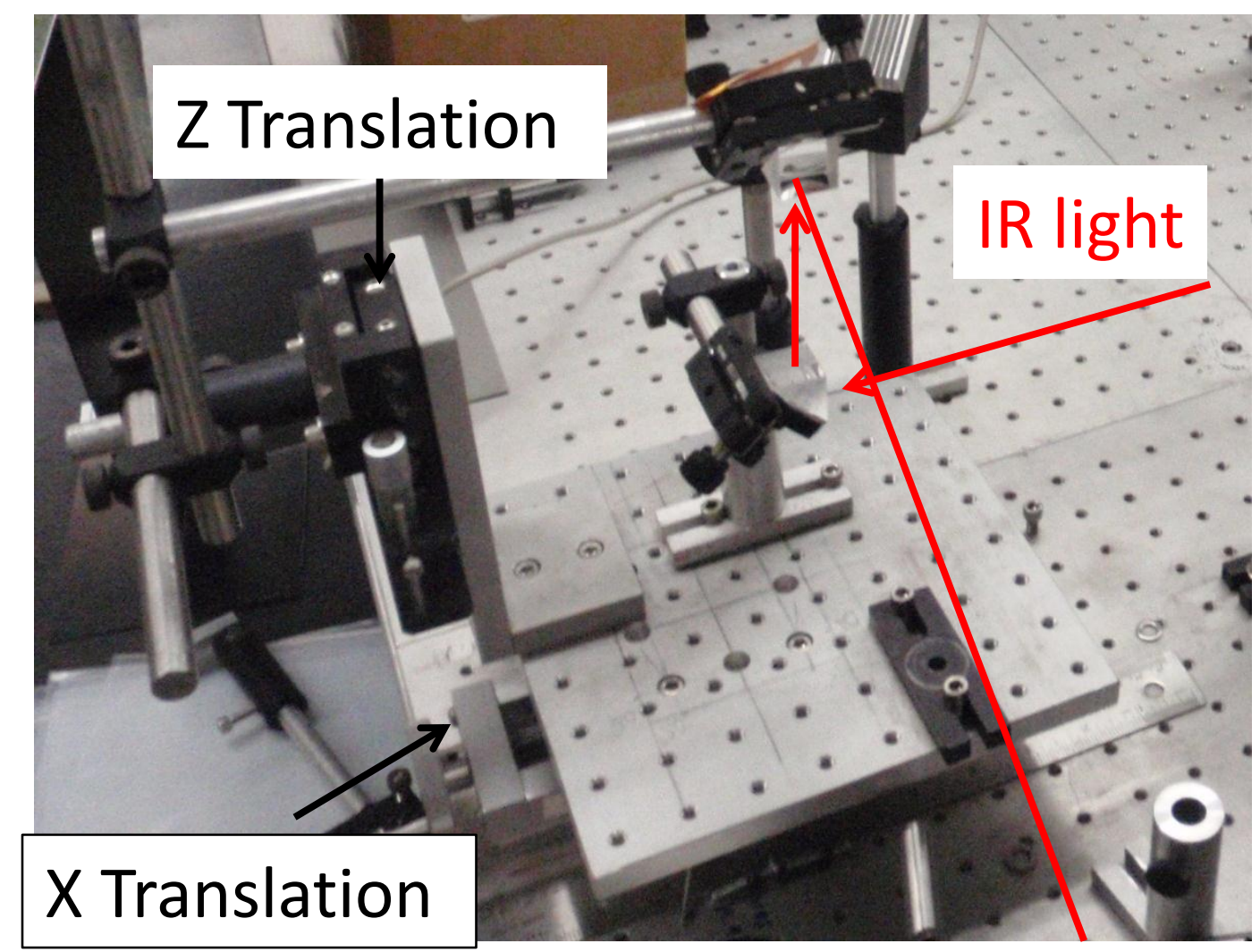

Figure 3.16 Translational stages for steering optics of the IR pump laser. The $X$ translation alters the distance of the laser beam relative to the surface. The $\mathrm{Z}$ translation raises and lowers the beam parallel to the surface. Not shown are the targets used for alignment, which lie on the optical ports of the surface scattering machine.

The steering optics mounted on translational stages, shown in Fig. 3.16, allow for two dimensions of translation which are totally independent. The laser beams can be reproducibly positioned to better than $0.2 \mathrm{~mm}$. This uncertainty results in errors of the velocity obtained from the TOF distributions on the order of $1 \%$.

Direct scattering - that is, molecules that do not trap - was the dominant mechanism found for all conditions used to obtain velocity measurement of $\mathrm{HCl}$. Indication for this direct scattering 
comes from a number of observations. One of the main indications of direct scattering is that molecules retain a "memory" of their initial velocities. That is the molecules with higher incidence energies of translation have higher final translational energy distributions. The final translational energy of the molecules also exceeds that for a Boltzmann distribution with a temperature corresponding to the surface temperature, indicative of direct scattering. A more detailed description of the final translational energies is given in section 4. Angular distributions for $\mathrm{HCl}(\mathrm{v}=2 \rightarrow 2)$ and $\mathrm{HCl}(\mathrm{v}=2 \rightarrow 1)$ were also measured under all conditions and showed narrow specular scattering.

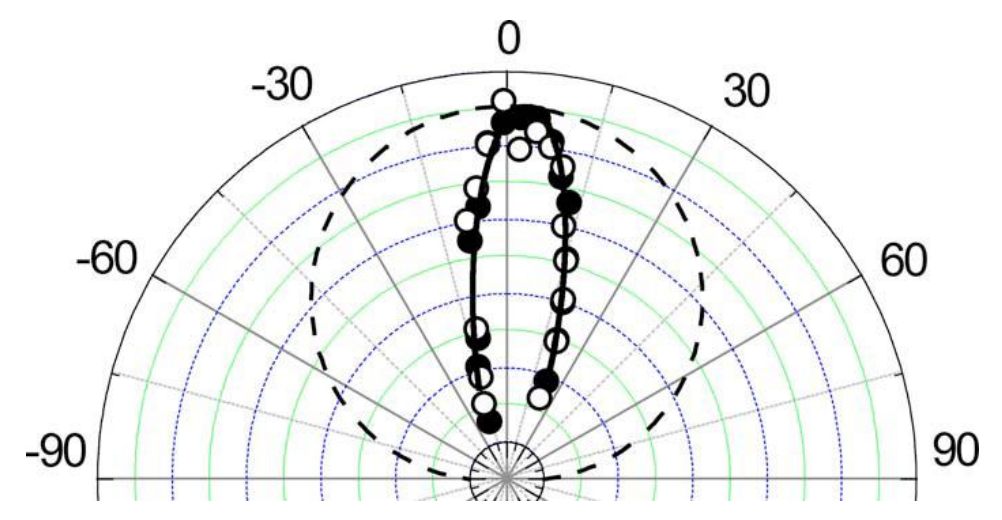

Figure 3.17 Angular distribution of $\mathrm{HCl}(\mathrm{v}=2 \rightarrow 2)$, open circles, and $\mathrm{HCl}(\mathrm{v}=2 \rightarrow 1)$, closed circles. The solid line indicates the best fit of $\cos ^{\mathrm{n}}\left(\theta-\theta_{0}\right)$, the dashed line $\cos (\theta)$, where $\theta_{0}$ is the specular angle, $3^{\circ} .{ }^{50}$

The angular distribution peaks at the specular angle. This combined with the narrow distribution, FWHM less than $30^{\circ}$, indicate a direct scattering mechanism. The solid line is a best fit of $\cos ^{n}$ where $n=28$. It is also worth noting that the angular distribution is independent of final vibrational state. The combination of the angular distribution, the "memory" of the velocity, and the scattered molecules having a velocity greater than that expected from Boltzman distribution indicate, that at all conditions measured here, the molecules scatter predominantly in a direct mechanism.

\subsection{Experimental details for NO vibrational excitation probabilities:}

These experiments took place using the same apparatus used for $\mathrm{HCl}$ translational energy measurements. Here, molecular beams were created by seeding NO in various carrier gases giving a range of incidence translational energies from $\mathrm{E}_{\mathrm{i}}=0.11$ to $1.05 \mathrm{eV}$. These molecules were scattered from a $\mathrm{Au}(111)$ crystal prepared in the same manner as in $\mathrm{HCl}$ experiments. Most experiments took place with normal incidence, with the exception of the data measured at $\mathrm{E}_{\mathrm{i}}=0.93 \mathrm{eV}$ where the incident angle was $3^{\circ}$ from surface normal. After scattering, NO molecules were state selectively ionized using (1+1) REMPI. UV light to ionize three vibrational states $\mathrm{NO}(\mathrm{v}=0,1,2)$ was produced by doubling the output of a tunable dye laser pumped with the third harmonic of a Nd:YAG in an analgous fashion to the $\mathrm{HCl}$ experiments. Rotationally resolved $(1+1)$ REMPI spectra of NO were recorded via the $A^{2} X^{+}\left(v^{\prime}=0\right) \leftarrow X^{2} \Pi(v=0,1,2)$ often referred to as the $\gamma_{\mathrm{v}, \mathrm{v}^{\prime}}$-bands. These bands were chosen because they are spectrally well isolated. UV light, 
$\sim 4 \mathrm{~mJ}$, of $\sim 225(\mathrm{v}=0), \sim 235(\mathrm{v}=1)$, and $\sim 250 \mathrm{~nm}(\mathrm{v}=2)$ was produced by using Coumarin 450, Coumarin 480, and a mixture of Coumarin 480 and Coumarin 503 respectively. The REMPI laser was not focused, as focusing led to a large background of non-resonant ionization. The ion detection was similar to that for the $\mathrm{HCl}$ experiments, but for all data except $\mathrm{E}_{\mathrm{i}}=0.93 \mathrm{eV}$ the MCP's were pulsed, for further details see section 3.6 In addition, the backing of the MCP's was changed from a power supply to grounding through a resistor. This allowed for more consistent control over the voltage on the back of the MCP, as often the power supply for supplying votage to the back of the MCP was overwhelmed by voltage leaking across the MCP from the power supply for the front. A capacitor was connected in parallel with the resistor in order to minimize ringing in the output from the MCP. The surface temperature was varied by resistive heating.

The velocity of all incident beams used in the NO study were measured in an analogous way to those in the $\mathrm{HCl}$ study. NO was pumped using IR light, $\lambda \sim 1.7 \mu \mathrm{m}$ via the second overtone transition, $\mathrm{NO}(\mathrm{v}=0 \rightarrow 3)$, and was ionized using REMPI. By knowing the distance between the lasers and altering the timing between the pump and probe lasers, the velocity and therefore the translational energy could be obtained in the manner explained in Appendix I. 


\section{Translational Energy Transfer:}

\subsection{Translational energy transfer in $\mathrm{HCl} / \mathrm{Au}(111)$ scattering:}

In non-adiabatic vibrational energy transfer, the vibrational degree of freedom is coupled to electron hole pairs in the metal. It has been shown that the translational energy has a large influence on the probability for both vibrational excitation and relaxation of various molecule surface systems. ${ }^{9,12,29,60}$ In most cases increasing the incidence energy of translation increases the transfer of vibrational energy $y^{9,12,29,60}$. However, the incidence energy dependence is thought not to arise due to coupling between the vibration of the molecule and its translation, but by altering the coupling strength/time with the electrons of the surface. ${ }^{29}$ Velocity changes have also not been noticed for large amplitude vibrational relaxation, indicating a "spectator view" of the translational degree of freedom. ${ }^{10}$ In this view, the translation is seen as a "spectator" in the vibrational energy transfer, affecting the coupling of the molecular vibration with electrons in the surface but not coupling directly to the vibration of the molecule. This stands in stark contrast to the most common electronically adiabatic mechanisms of vibrational energy transfer at surfaces where vibration to translation (V-T) coupling is essential. ${ }^{30}$

However, the validity of this "spectator claim" remains experimentally untested. Most studies of vibrational energy transfer have lacked sufficient translational energy resolution to test this. ${ }^{10,12}$ The number of studies existing in this area is limited, even ignoring these limitations. I decided to test the "spectator view" for vibrational relaxation of $\mathrm{HCl}(\mathrm{v}=2 \rightarrow 1)$ scattering from $\mathrm{Au}(111)$. As seen in section 2.3, $\mathrm{HCl}$ exhibits a combination of adiabatic and nonadiabatic mechanisms for vibrational excitation ${ }^{12}$, which makes $\mathrm{HCl}$ an excellent candidate to check the validity of the translation as a spectator.

Another driving force for this study is the prevailing thought that translational energy of molecules scattering from surfaces behaves in a purely mechanical manner. That is, the scattered translational energy is determined by momentum conservation of the incident molecule and the surface, where the surface acts as not as a single atom but as a molecule with an effective mass of one to several atoms, with the total effective mass dependent on the stiffness of the surface $^{61,62}$ I also studied translational energy transfer of vibrationally elastic $\mathrm{HCl}$ scattering from the $\mathrm{Au}(111)$ surface. Translational energy transfer of vibrationally elastic scattering was also probed as a function of incidence vibrational states, for $\mathrm{HCl}(\mathrm{v}=0)$ and $\mathrm{HCl}(\mathrm{v}=2)$.

\subsection{Incident and scattered beam quantification:}

Rotationally resolved spectra for scattered $\mathrm{HCl}(\mathrm{v}=2)$ and $\mathrm{HCl}(\mathrm{v}=1)$ molecules were recorded using several previously reported bands. ${ }^{63}$ The molecules were prepared into $\mathrm{HCl}(\mathrm{v}=2 \mathrm{~J}=1)$ by overtone R(0) IR excitation, and probed with tunable UV light in the configuration shown in Fig. 3.15(B). In this configuration the REMPI laser is only sensitive to scattered molecules. The time delay between the lasers is set for maximum signal. Representative spectra are shown in Fig. 4.1. 


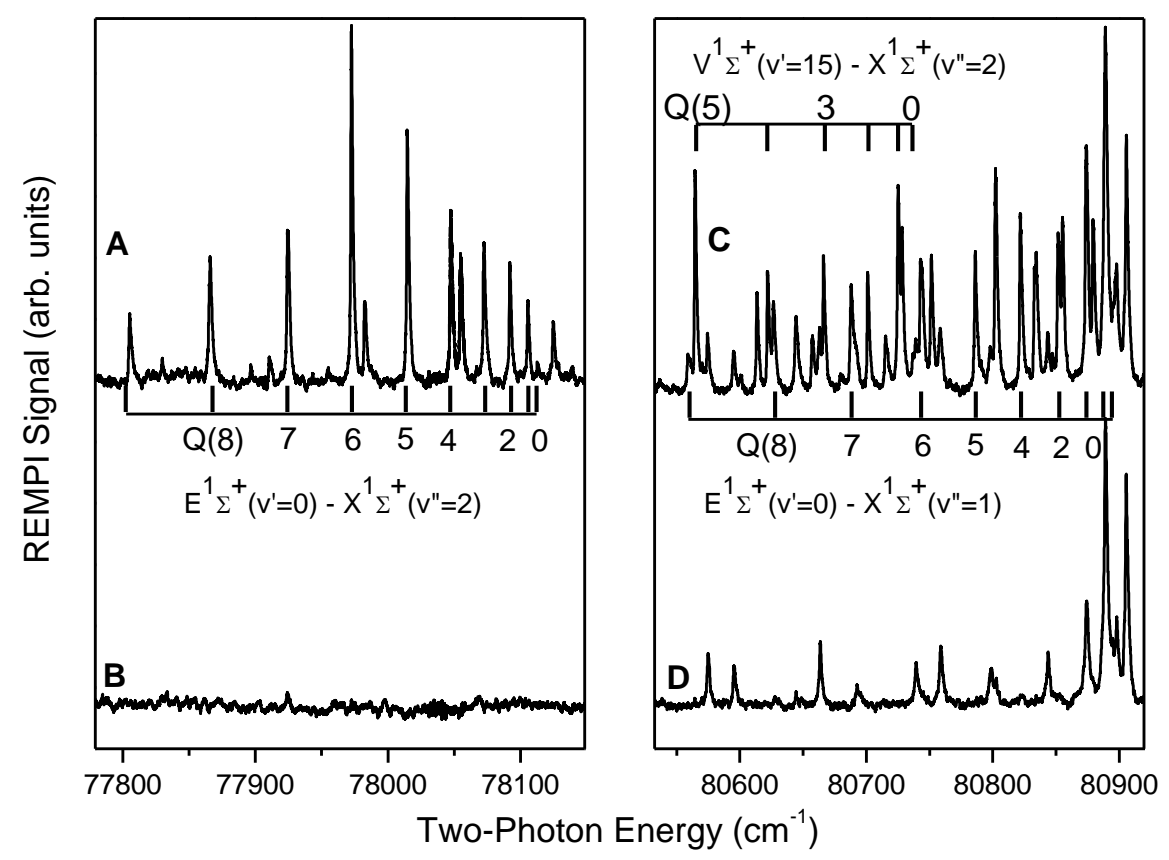

Figure 4.1 2+1 REMPI spectra for scattered $\mathrm{HCl}$. A and $\mathrm{C}$ were recorded with the IR laser turned on, $\mathrm{B}$ and $\mathrm{D}$ with the IR laser off. A and B contain the $E^{l} \Sigma^{+}\left(v^{\prime}=0\right)-X^{l} \Sigma^{+}\left(v^{\prime \prime}=2\right)$ band (Q-branch indicated by the comb) used to monitor the $\mathrm{v}=2$ signal. $\mathrm{C}$ and $\mathrm{D}$ contain the $E^{l} \Sigma^{+}\left(v^{\prime}=0\right)-X^{l} \Sigma^{+}\left(v^{\prime \prime}=1\right)$ band used to monitor $\mathrm{v}=1$ signal as well as $V^{l} \Sigma^{+}\left(v^{\prime}=15\right)-X^{l} \Sigma^{+}\left(v^{\prime \prime}=2\right)$ (Q-branch indicated by the comb). The spectra were recorded for $\mathrm{E}_{\mathrm{i}}=0.52 \mathrm{eV}$ and $\mathrm{T}_{\mathrm{S}}=300$ $\mathrm{K}$. The delay between the two lasers was $12 \mu \mathrm{s}^{50}$

The spectra shown in Fig. 4.1 indicate that all of the scattered $\mathrm{HCl}(\mathrm{v}=2)$ and the majority of scattered $\mathrm{HCl}(\mathrm{v}=1)$ are created from incident beam $\mathrm{HCl}(\mathrm{v}=2)$ molecules. Figure 4.1(A) shows rotationally inelastic scattering of $\mathrm{HCl}(\mathrm{v}=2)$. Figure 4.1(C) shows predominantly vibrationally inelastic scattering where $v=2$ relaxes upon interaction with the surface to form $v=1$. Despite the fact that the $E^{l} \Sigma^{+}\left(\mathrm{v}^{\prime}=0\right)-X^{l} \Sigma^{+}\left(\mathrm{v}^{\prime \prime}=1\right)$ band is partially overlapped by the $V^{l} \Sigma^{+}\left(\mathrm{v}^{\prime}=15\right)$ $X^{l} \Sigma^{+}\left(\mathrm{v}^{\prime \prime}=2\right)$ band it can still be clearly resolved. Figure 4.1(D) shows $\mathrm{v}=1$ with the pump laser off, the weak spectral features are assigned to the thermally populated $v=1$ in the incident beam which is rotationally inelastically scattered from the surface and can easily be subtracted from the results in Figure 4.1(C). Utilizing these bands allows us to probe the translational energy in any relevant vibrational and rotational state.

As described previously by varying the time delay between the two lasers and knowing the distance between the two lasers I can measure the velocity of the molecules. Figure 3.15(A) shows the configuration for measuring the translational energy of the incident beam. Raw TOF data for different mixtures is shown in Fig. 4.2. 


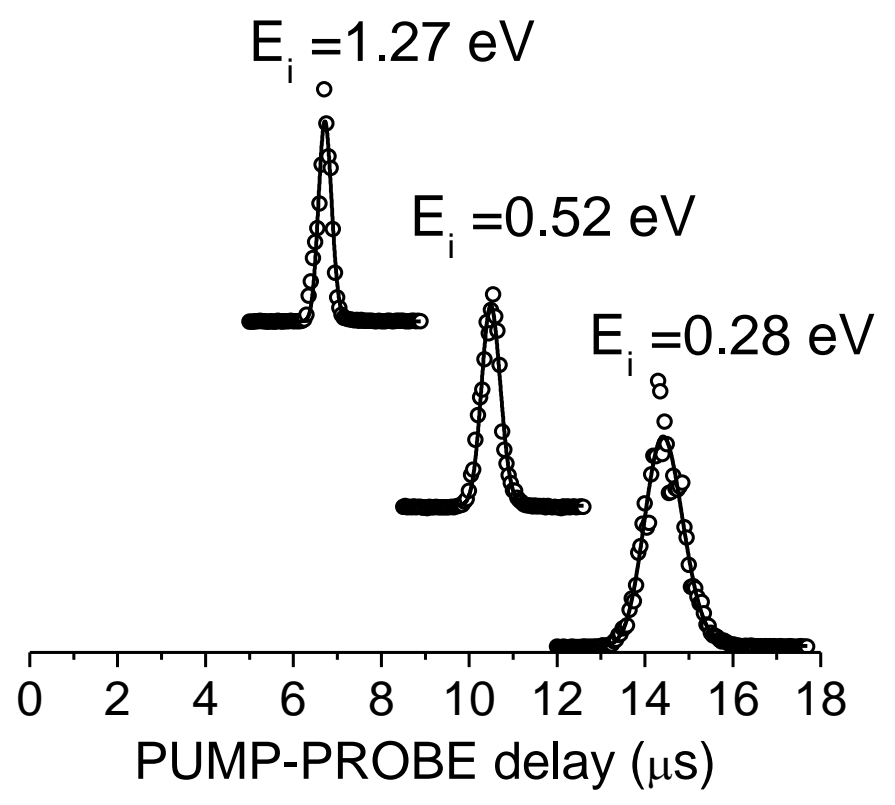

Figure 4.2 TOF spectra of the optically prepared $\mathrm{HCl}(\mathrm{v}=2)$ incident beam at three incident translational energies $1 \%$ $\mathrm{HCl} / \mathrm{H}_{2}$ with $\mathrm{E}_{\mathrm{i}}=1.27 \mathrm{eV} ; 10 \% \mathrm{HCl} / \mathrm{H}_{2}$ with $\mathrm{E}_{\mathrm{i}}=0.52 \mathrm{eV} ; 10 \% \mathrm{HCl} / 20 \% \mathrm{~N}_{2} / 70 \% \mathrm{D}_{2}$ with $\mathrm{E}_{\mathrm{i}}=0.28 \mathrm{eV}$ measured by changing the delay between the IR PUMP laser pulse and the UV PROBE laser pulse. The IR laser pumped the R(0) line of the overtone band. The UV laser was tuned to probe the $E^{l} \Sigma^{+}\left(v^{\prime}=0\right)-X^{l} \Sigma^{+}\left(v^{\prime \prime}=2\right) \mathrm{Q}(1)$ line. The open circles are experimental data-points and the solid lines are fits to Eq. (4.1) in time space Eq. (A 1.11). ${ }^{50}$

The spread in the arrival times, $\delta \mathrm{t} / \mathrm{t}$, and consequently the spread in the velocity, $\delta \mathrm{v} / \mathrm{v}$, was found to be around $6-7 \%$. The beams were characterized by converting raw TOF data to velocity space and fitting with

$$
P(v)=A v^{3} e^{-\left(\frac{v-v_{0}}{\alpha}\right)^{2}}
$$

where $\mathrm{A}$ is a normalization constant, $v_{0}$ is a stream velocity, and $\alpha$ is a width parameter.

Transformations of Eq. (4.1) are shown in Appendix I. Table 4.1 shows the results for the fitting parameters and the average translational energy for the 6 gas mixtures used. 
Table 4.1 Parameters describing the speed distributions for the incident beam.

\begin{tabular}{|l|l|l|l|l|}
\hline Mixing Ratios & $\left\langle E_{i}\right\rangle, \mathrm{eV}$ & $\sqrt{\mid\left\langle E_{i}^{2}\right\rangle-\left\langle E_{i}\right\rangle^{2}}, \mathrm{eV}$ & $v_{0}, \mathrm{~m} / \mathrm{s}$ & $\alpha, \mathrm{m} / \mathrm{s}$ \\
\hline \hline $10 \% \mathrm{HCl} / 20 \% \mathrm{~N}_{2} / 70 \% \mathrm{D}_{2}$ & 0.275 & 0.017 & 1210 & 52 \\
\hline $10 \% \mathrm{HCl} / 10 \% \mathrm{~N}_{2} / 80 \% \mathrm{D}_{2}$ & 0.317 & 0.021 & 1297 & 60 \\
\hline $10 \% \mathrm{HCl} / 90 \% \mathrm{D}_{2}$ & 0.447 & 0.027 & 1542 & 67 \\
\hline $10 \% \mathrm{HCl} / 90 \% \mathrm{H}_{2}$ & 0.520 & 0.021 & 1665 & 48 \\
\hline $5 \% \mathrm{HCl} / 95 \% \mathrm{H}_{2}$ & 0.779 & 0.062 & 2031 & 114 \\
\hline $2.5 \% \mathrm{HCl} / 97.5 \% \mathrm{H}_{2}$ & 0.974 & 0.059 & 2276 & 98 \\
\hline $1 \% \mathrm{HCl} / 99 \% \mathrm{H}_{2}$ & 1.268 & 0.056 & 2601 & 81 \\
\hline
\end{tabular}

This velocity spread, which is inherent in molecular beams, has two negative effects on the current work. First, the spread limits the ability to determine exactly the velocity change resulting from the collision with the surface. Second after the molecules are excited they spread out due to the inherent velocity spread in the incident beam. This leads to uncertainty in the time the molecules strike the surface. To minimize this uncertainty the IR pump laser is placed as close to the sample as possible, $\sim 3 \mathrm{~mm}$, and focused. In regards to the first problem I have made experiments to check the velocity spread effect on my data. In order to do this I collected TOF data at several distances from the surface at the specular angle. These results are plotted as the energy probability distributions obtained by direct inversion of the time of flight data using the proper Jacobian (see Appendix I), and the method of obtaining these distributions will be discussed later. The point here is to focus only on the change in the translational energy distribution. 


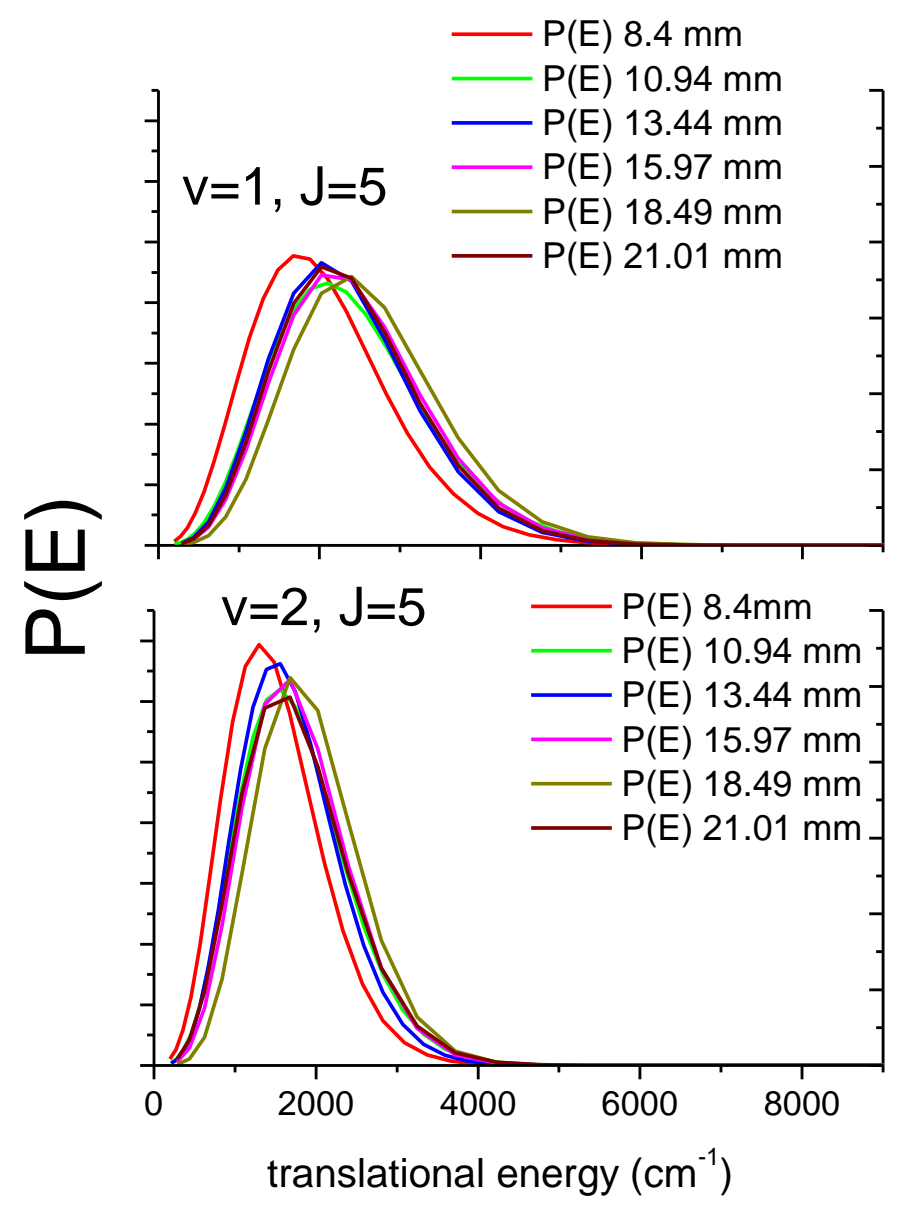

Figure 4.3 Translational energy distributions as a function of distance from the surface from $8.4 \mathrm{~mm}$ to $21 \mathrm{~mm}$ for $\mathrm{HCl}(\mathrm{v}=2 \mathrm{~J}=1 \rightarrow \mathrm{v}=1 \mathrm{~J}=5)$, top and $\mathrm{HCl}(\mathrm{v}=2 \mathrm{~J}=1 \rightarrow \mathrm{v}=2 \mathrm{~J}=5)$, bottom.

With the exception of the $8.4 \mathrm{~mm}$ flight distance, which deviated for unknown reasons, all of the results obtained at different distances appear similar, varying only slightly within the error inherent in the measurement. Probably the main factor in this error is the uncertainty in the flight distance, which would only have to vary by $0.5 \mathrm{~mm}$ to lead to the difference in the $\mathrm{P}(\mathrm{E})$ shown in Fig. 4.3. 


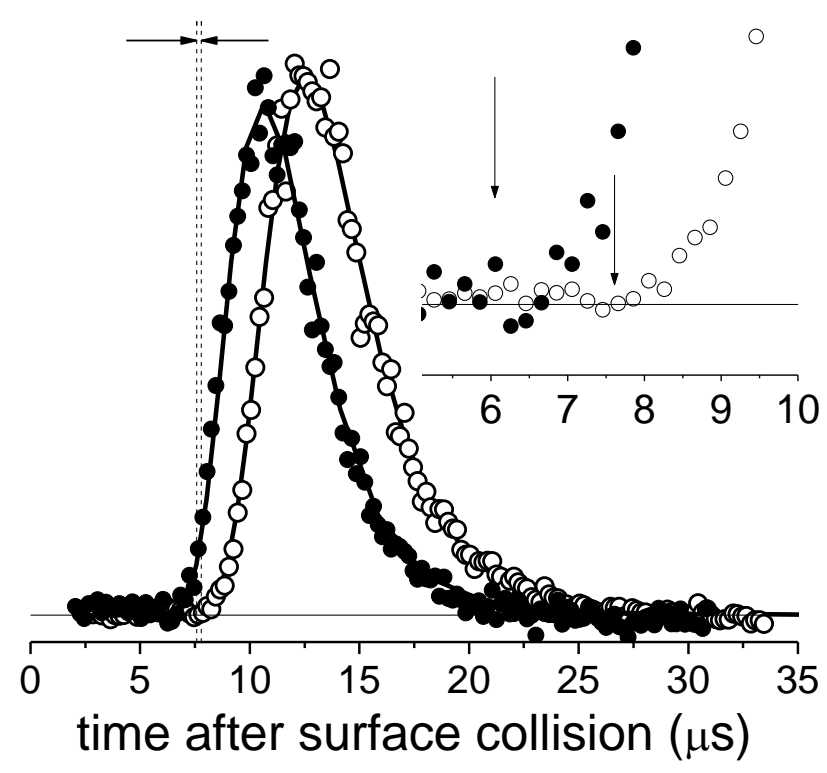

Figure 4.4 Two normalized TOF distributions, vibrationally elastic, $\mathrm{HCl}(\mathrm{v}=2 \rightarrow 2)$, scattering, empty circles, vibrational inelastic, $\mathrm{HCl}(\mathrm{v}=2 \rightarrow 1)$, scattering, filled circles, are shown at $\mathrm{E}_{\mathrm{i}}=0.52 \mathrm{eV}$ and $\mathrm{T}_{\mathrm{s}}=300 \mathrm{~K}$. The solid lines are fits to equation 4.1 but in time space. The vertical dashed lines represent the arrival time if the incident beam underwent translationally elastic scattering. The inset is a blow up of the leading edge of the signal. The two arrows represent the earliest possible arrival time if all the vibrational energy lost by the molecule were transferred to translation of the molecule, assuming the thermal phonon-to-translation or electron-hole-pair-to-translation contribution from the surface is negligible. ${ }^{50}$

Figure 4.4 shows two representative TOF distributions for vibrationally elastic $(\mathrm{HCl}(\mathrm{v}=2 \rightarrow 2)$, empty circles) and inelastic $(\mathrm{HCl}(\mathrm{v}=2 \rightarrow 1)$, filled circles) collisions. The TOF are normalized to the peak intensity. In both cases, the scattered molecules' TOF distribution is significantly broadened by the interactions experienced in the collision with the surface. The TOF data have small amount of signal near the energetic maximum, but most molecules undergo a significant translational energy loss at the surface.

It is clear from the TOF data that vibrationally inelastic events $\mathrm{HCl}(\mathrm{v}=2 \rightarrow 1)$ result in (on average) earlier arrival times than do vibrationally elastic scattering events $\mathrm{HCl}(\mathrm{v}=2 \rightarrow 2)$. This shows directly the presence of vibration-translation coupling. The shift in arrival time is small, and later the fraction of energy coupled between vibration of the molecule and the translational energy of the molecule will be discussed. The same measurements were carried out over a range of incidence energies. The raw TOF data is shown in Fig. 4.5. 


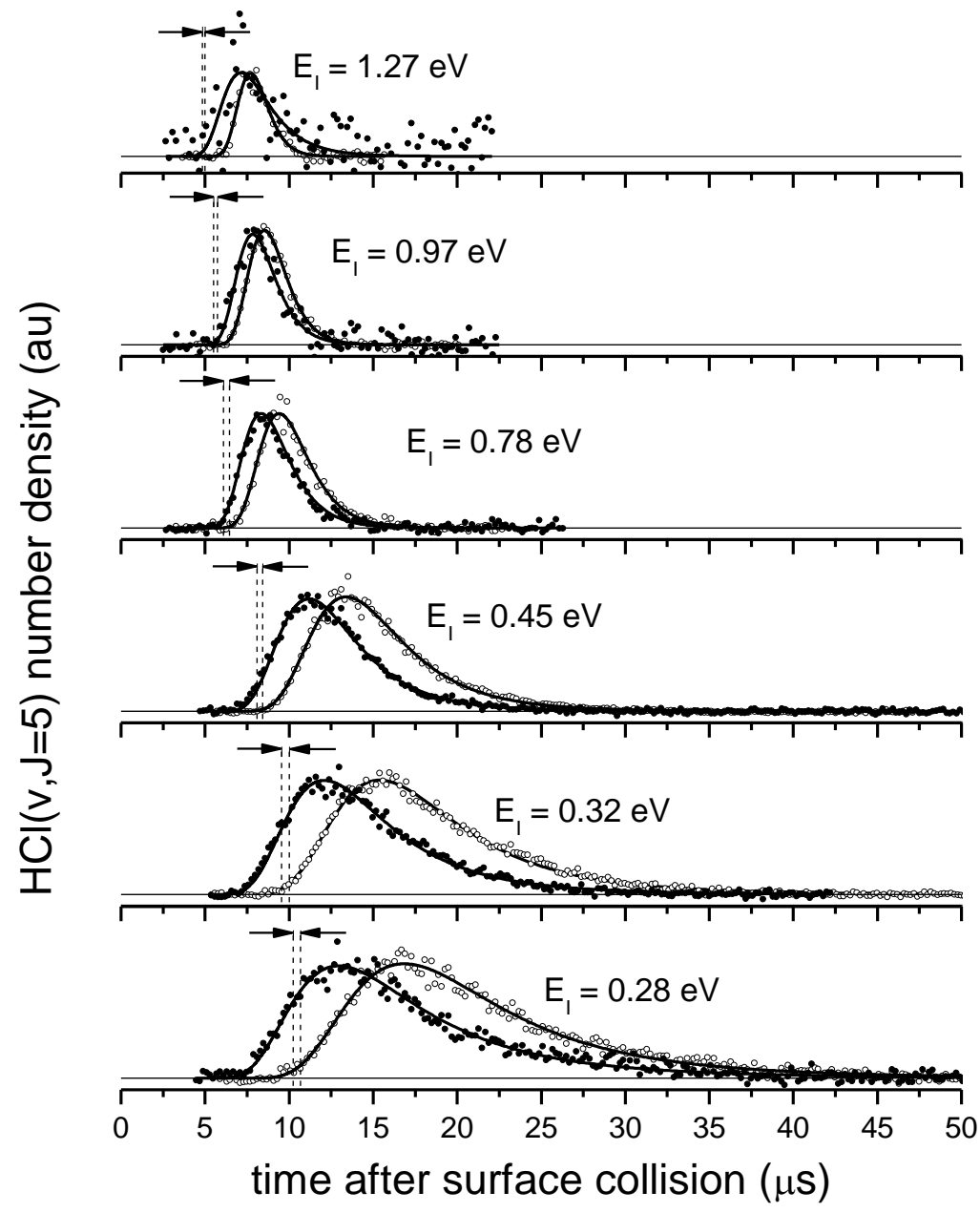

Figure 4.5 Normalized TOF data taken for vibrationally inelastic $\mathrm{HCl}(\mathrm{v}=2 \rightarrow 1)$, empty circles, and elastic $\mathrm{HCl}(\mathrm{v}=2 \rightarrow 2)$, filled circles, scattering in an analogous manner to that in Fig. 4.4 at incidence translational energies of $1.27,0.97,0.78,0.45,0.32$, and $0.28 \mathrm{eV}$ respectively $\mathrm{T}_{\mathrm{s}}=300 \mathrm{~K}$. See Fig. 4.4 for additional information. ${ }^{50}$

The incidence energy of translation spans a range from 0.28 to $1.27 \mathrm{eV}$. The elastic and inelastic TOF data are again normalized to their peak intensities. The final translational energy increases with increasing incidence translational energy, which as previously mentioned is indicative of a direct scattering mechanism, i.e. a memory effect. In all cases the vibrationally inelastic scattering arrives slightly earlier than the vibrationally elastic case indicative of V-T coupling.

The intensities shown in Fig. 4.5 of the outgoing elastic and inelastic channels do not indicate a relative population of these two states. While a thorough examination of angular, TOF, and rotational distributions is needed to quantify accurately the relaxation probabilities, the intensity of the rotational spectra indicates that after scattering, roughly equal amounts of $\mathrm{HCl}(\mathrm{v}=2)$ and $\mathrm{HCl}(\mathrm{v}=1)$ are present in the beam. From this we can estimate that the survival probability of 
$\mathrm{HCl}(\mathrm{v}=2)$ when scattered from the surface is less than $50 \%$, as it is unknown how much relaxes to the ground vibrational state $\mathrm{HCl}(\mathrm{v}=2 \rightarrow 0)$, due to the large background of $\mathrm{HCl}(\mathrm{v}=0)$ in the molecular beam. More carefully executed studies of survival probability are needed.

\subsection{Translational energy exchange:}

By directly inverting the TOF data into translational energy probability distributions in a manner described in Appendix I, we can quantify the scattered molecules in a similar manner to the incident beams (Table 4.1). Typical derived translational energy distributions are shown in Fig. 4.6.

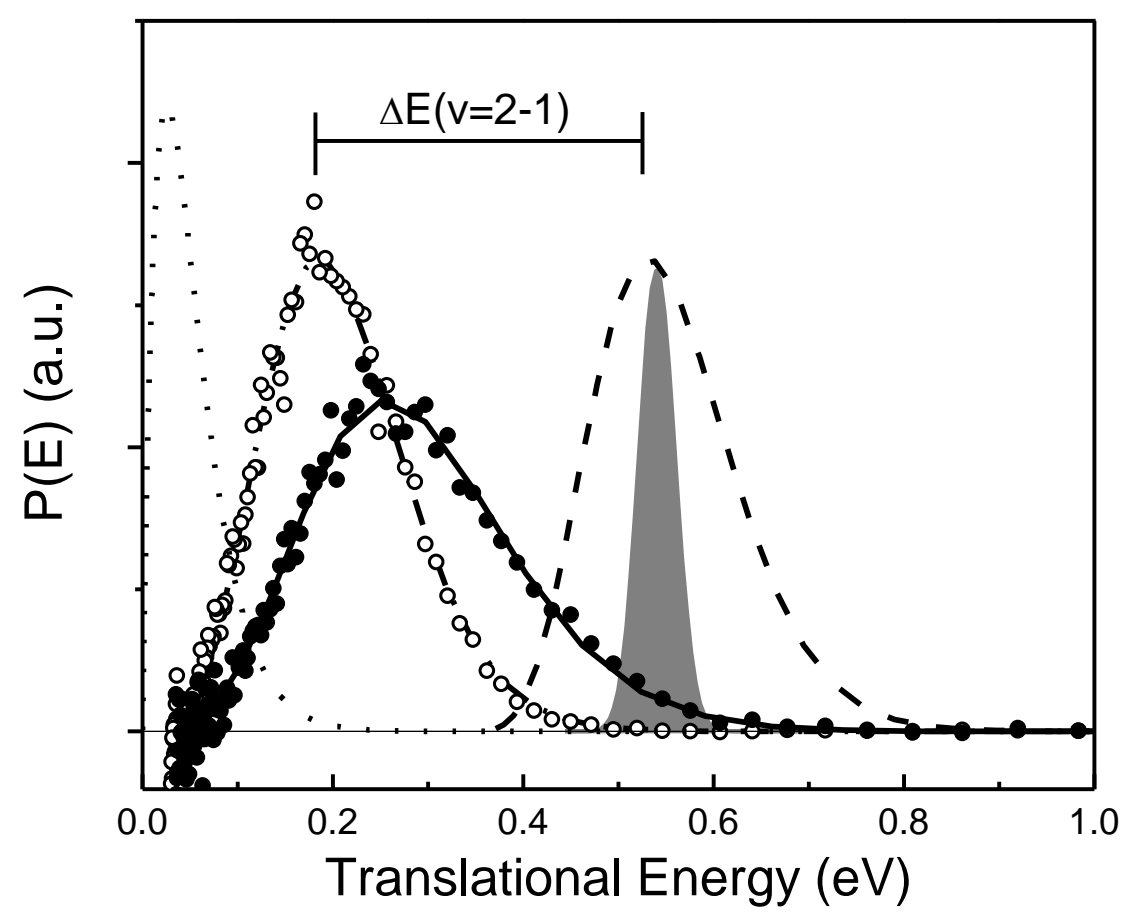

Figure 4.6 Translational energy distributions for $\mathrm{HCl}(\mathrm{v}=2 \mathrm{~J}=1 \rightarrow \mathrm{v}=2 \mathrm{~J}=5)$, open circles, $\mathrm{HCl}(\mathrm{v}=2 \mathrm{~J}=1 \rightarrow \mathrm{v}=1 \mathrm{~J}=5)$, closed circles at $\mathrm{E}_{\mathrm{i}}=0.52 \mathrm{eV}$ and $\mathrm{T}_{\mathrm{s}}=300 \mathrm{~K}$. The solid lines are fits as described in Appendix 1. The dotted line corresponds to a Boltzmann distribution at $300 \mathrm{~K}$. The shaded region corresponds to the incident beam and the dashed line is $\operatorname{HCl}(\mathrm{v}=2 \mathrm{~J}=1 \rightarrow \mathrm{v}=2 \mathrm{~J}=5)$, shifted by the vibrational energy difference between $\mathrm{HCl}(\mathrm{v}=2 \mathrm{~J}=5)$ and $\mathrm{HCl}(\mathrm{v}=1 \mathrm{~J}=5)$, that is if all the vibrational energy lost coupled to translation we would expect $\mathrm{HCl}(\mathrm{v}=1 \mathrm{~J}=5)$ energy distribution to lay here. ${ }^{50}$

Several things are clear from plotting the translational energy distributions. First, the translational energy of the scattered molecules, in both the vibrationally elastic and inelastic cases, exceeds a Boltzmann distribution at $300 \mathrm{~K}$. Both transfer a significant fraction of their initial translational energy to the surface. Compared to vibrationally elastic, inelastic scattering shows a broader translational energy distribution which is also shifted to higher energies. The, naïve view that all the vibrational energy lost would be transferred either to the surface, or to the 
translational energy of the outgoing molecule is not observed. If all of the lost vibrational energy were transferred to translational energy of the molecule, we would expect the $\mathrm{HCl}(\mathrm{v}=1 \mathrm{~J}=5)$ translational energy distribution to be represented by the dashed line in Fig. 4.6, which is clearly much more energetic than the observed distribution. Equally clear is that the translation is not simply a spectator, for if this were the case the translational energy distribution would be the same for the vibrationally elastic and inelastic cases. Results similar to those shown in Fig. 4.6 have been made at six other incidence translational energies ranging from $\mathrm{E}_{\mathrm{i}}=0.28$ to $1.27 \mathrm{eV}$, and for several outgoing rotational states $(\mathrm{J}=1-9)$, and several surface temperatures (at two incidence translational energies) for both the vibrationally elastic and inelastic cases.

The average translational energy, derived from scattered translational energy distributions like that shown in Fig. 4.6, and the parameters that characterize their speed distributions are shown in Table 4.2, for the vibrationally elastic case, and Table 4.3, for the vibrationally inelastic case. The tables refer to scattering from a room temperature surface, and a final rotational state of $\mathrm{J}=5$. 
Table 4.2 Parameters describing the speed distributions for the vibrationally elastically scattered $\mathrm{HCl}(\mathrm{v}=2 \mathrm{~J}=1 \rightarrow \mathrm{v}=2$ $\mathrm{J}=5)^{50}$

\begin{tabular}{|l|c|c|c|c|c|}
\hline \multicolumn{1}{|c|}{ Mixing Ratios } & $\left\langle E_{i}\right\rangle, \mathrm{eV}$ & $\left\langle E_{s}\right\rangle, \mathrm{eV}$ & $\sqrt{\left\langle E_{s}^{2}\right\rangle-\left\langle E_{s}\right\rangle^{2}}, \mathrm{eV}$ & $v_{0}, \mathrm{~m} / \mathrm{s}$ & $\alpha, \mathrm{m} / \mathrm{s}$ \\
\hline \hline $10 \% \mathrm{HCl} / 20 \% \mathrm{~N}_{2} / 70 \% \mathrm{D}_{2}$ & 0.275 & 0.19183 & 0.1135 & 488 & 526 \\
\hline $10 \% \mathrm{HCl} / 10 \% \mathrm{~N}_{2} / 80 \% \mathrm{D}_{2}$ & 0.317 & 0.21862 & 0.1102 & 737 & 445 \\
\hline $10 \% \mathrm{HCl} / 90 \% \mathrm{D}_{2}$ & 0.447 & 0.24568 & 0.1036 & 922 & 375 \\
\hline $10 \% \mathrm{HCl} / 90 \% \mathrm{H}_{2}$ & 0.520 & 0.27871 & 0.1148 & 995 & 389 \\
\hline $5 \% \mathrm{HCl} / 95 \% \mathrm{H}_{2}$ & 0.779 & 0.47 & 0.1572 & 1408 & 396 \\
\hline $2.5 \% \mathrm{HCl} / 97.5 \% \mathrm{H}_{2}$ & 0.974 & 0.52517 & 0.1548 & 1535 & 363 \\
\hline $1 \% \mathrm{HCl} / 99 \% \mathrm{H}_{2}$ & 1.268 & 0.61473 & 0.2431 & 1511 & 549 \\
\hline
\end{tabular}

Table 4.3 Parameters describing the speed distributions for the vibrationally inelastically scattered $\mathrm{HCl}(\mathrm{v}=2$ $\mathrm{J}=1 \rightarrow \mathrm{v}=1 \mathrm{~J}=5)^{50}$

\begin{tabular}{|l|c|c|c|c|c|}
\hline \multicolumn{1}{|c|}{ Mixing Ratios } & $\left\langle E_{i}\right\rangle, \mathrm{eV}$ & $\left\langle E_{s}\right\rangle, \mathrm{eV}$ & $\sqrt{\left\langle E_{s}^{2}\right\rangle-\left\langle E_{s}\right\rangle^{2}}, \mathrm{eV}$ & $v_{0}, \mathrm{~m} / \mathrm{s}$ & $\alpha, \mathrm{m} / \mathrm{s}$ \\
\hline $10 \% \mathrm{HCl} / 20 \% \mathrm{~N}_{2} / 70 \% \mathrm{D}_{2}$ & 0.275 & 0.1126 & 0.0608 & 475 & 351 \\
\hline $10 \% \mathrm{HCl} / 10 \% \mathrm{~N}_{2} / 80 \% \mathrm{D}_{2}$ & 0.317 & 0.13485 & 0.0657 & 605 & 334 \\
\hline $10 \% \mathrm{HCl} / 90 \% \mathrm{D}_{2}$ & 0.447 & 0.17131 & 0.0707 & 780 & 305 \\
\hline $10 \% \mathrm{HCl} / 90 \% \mathrm{H}_{2}$ & 0.520 & 0.20261 & 0.0733 & 900 & 285 \\
\hline $5 \% \mathrm{HCl} / 95 \% \mathrm{H}_{2}$ & 0.779 & 0.37067 & 0.1162 & 1272 & 327 \\
\hline $2.5 \% \mathrm{HCl} / 97.5 \% \mathrm{H}_{2}$ & 0.974 & 0.44436 & 0.1180 & 1439 & 299 \\
\hline $1 \% \mathrm{HCl} / 99 \% \mathrm{H}_{2}$ & 1.268 & 0.53535 & 0.1234 & 1609 & 282 \\
\hline
\end{tabular}

\subsection{Rotational state and surface temperature effects:}

It is important to understand the role of both the rotational degree of freedom in $\mathrm{HCl}$, as well as the thermal lattice motion of the $\mathrm{Au}(111)$ surface. Before focusing on the coupling between vibration of the molecules and electronic excitation of the metal, I would like to discuss the effect final rotational state and surface temperature have on the scattered translational energy distributions..

Rotational excitation as seen in Fig. 4.1 is not particularly high in energy for either the $\mathrm{HCl}(\mathrm{v}=2 \rightarrow 1)$ or $\mathrm{HCl}(\mathrm{v}=2 \rightarrow 2)$ channel. In addition the $\mathrm{TOF}$ distributions are measured at a specific rotational state so that the rotational energy change of the molecule is well defined. For both the vibrationally elastic and inelastic cases, translational energy distributions were recorded 
as a function of final rotational state. $\mathrm{J}=1$ represents rotationally elastic scattering, as the molecules were optically pumped to $\mathrm{HCl}(\mathrm{v}=2 \mathrm{~J}=1)$ in the incident beam.

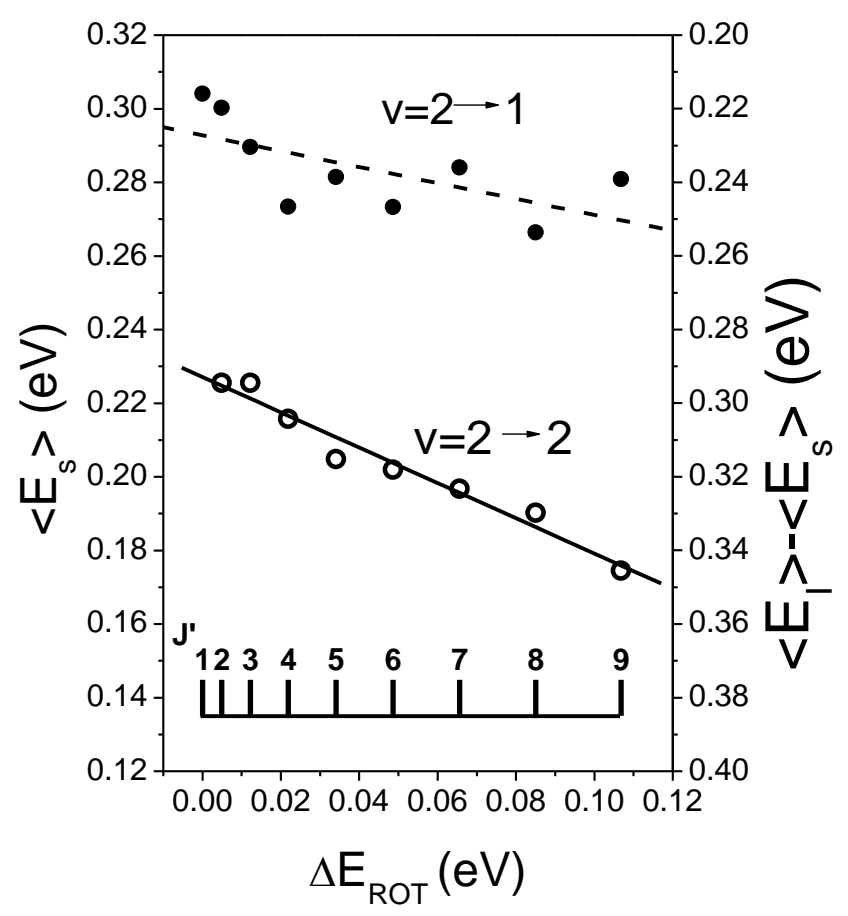

Figure 4.7 Average translational energy of the scattered molecules as a function of rotational energy for vibrationally elastic, $\mathrm{HCl}(\mathrm{v}=2 \rightarrow 2)$ empty circles, and inelastic $\mathrm{HCl}(\mathrm{v}=2 \rightarrow 1)$ scattering, filled circles at $\mathrm{E}_{\mathrm{i}}=0.52 \mathrm{eV}$ and $T_{s}=300 \mathrm{~K}$. The dashed and solid lines are empirical fits to the data. The comb represents the final rotational state of the outgoing molecules. Note that the change in rotational energy is larger than the corresponding loss in translational energy.

It is clear from Fig. 4.7 that the translation of the molecule couples to the rotation of the molecule upon interacting with the surface. The coupling is weaker in the vibrationally inelastic case as indicated by the smaller slope of the line in Fig. 4.7. The coupling in either case is not particularly strong, certainly not strong enough to account for the drastic total energy loss seen when molecules scatter from the surface. The coupling between rotation and translation will be discussed more in detail below.

Simply stated, Fig. 4.7 shows that scattering channels with more rotational energy exhibit reduced translational energy, both in the vibrationally elastic, empty circles and inelastic, filled circles cases. If the energy uptake by the solid were independent of final rotational state, the translational energy would decrease by the amount of rotational excitation. That is, a decrease in $\left\langle\mathrm{E}_{\mathrm{s}}\right\rangle$ from one J-state to another would be perfectly compensated by the excess rotational excitation. However the coupling between the translation of the molecule and its rotation is 
incomplete. For example at for $\mathrm{HCl}(\mathrm{v}=2 \mathrm{~J}=1 \rightarrow \mathrm{v}=2 \quad \mathrm{~J}=9)$ the change in rotational energy corresponds to more than $0.1 \mathrm{eV}$, however only a $0.05 \mathrm{eV}$ decrease in translational energy is seen. This is clear evidence of rotational anti-correlation with translational inelasticity. In other words, the surface excitation accompanying the scattering process $\mathrm{HCl}(\mathrm{v}=2 \mathrm{~J}=1 \rightarrow 2 \mathrm{~J}=9)$ is 0.05 $\mathrm{eV}$ less than that accompanying the $\mathrm{HCl}(\mathrm{v}=2 \mathrm{~J}=1 \rightarrow 2 \mathrm{~J}=1)$ channel.

This effect is quite subtle and to better understand it I developed the following mathematical description. For the vibrationally elastic channel, the linear relation between outgoing translational and rotational excitation energy is as follows:

$$
\left\langle E_{s}\right\rangle=-\alpha \Delta E_{\text {ROT }}+0.23 \text {, where energies are in } \mathrm{eV} .
$$

As the amount of surface excitation, rotational excitation, and final translation energy must sum to the initial incidence energy:

$$
E_{i}=\left\langle E_{s}\right\rangle+\Delta E_{R O T}+E_{S U R F},
$$

one may also express the degree of surface excitation in terms of $\Delta \mathrm{E}_{\mathrm{ROT}}$ :

$$
E_{S U R F}=E_{i}-\left\langle E_{S}\right\rangle-\Delta E_{R O T}=E_{i}+(\alpha-1) \Delta E_{R O T}-0.23
$$

From this, it is convenient to describe the correlation between rotational and surface excitation as a partial derivative:

$$
\frac{\partial E_{S U R F}}{\partial \Delta E_{\text {ROT }}}=\alpha-1
$$

As was mentioned above, if $\alpha=1$, the anti-correlation disappears and the translational energy scales directly with rotational excitation. Within this formalism, we may also characterize the rotational anti-correlation for the vibrationally inelastic channel, also shown in Fig. 4.7. The numerical results from this analysis are as follows:

$$
\left(\frac{\partial E_{\text {SURF }}}{\partial \Delta E_{\text {ROT }}}\right)_{\text {vibraionaly ylassic }}=0.48-1=-0.52 \text { and }\left(\frac{\partial E_{\text {SURF }}}{\partial \Delta E_{\text {ROT }}}\right)_{\text {vibratioally inelastic }}=0.22-1=-0.78
$$

In summary, we observe an anti-correlation of translational inelasticity with rotational excitation. This anti-correlation is stronger for the vibrationally inelastic channel.

Anti-correlation of this type has been observed previously for $\mathrm{NO} / \mathrm{Ag}(111)^{64}, \mathrm{NO} / \mathrm{Ge}^{65}$, $\mathrm{N}_{2} / \mathrm{Ag}(111)^{66}$ and $\mathrm{N}_{2} / \mathrm{Cu}(110)^{67}$ systems. In these systems, the effect was attributed to the reduction in the effective mass of the molecule and this reduction in mass should lead to a reduction in the translational energy transferred to the surface. ${ }^{68}$ It is probable that a similar explanation is applicable here. 
There is, however, an additional feature of my results that is not anticipated by the previous work on ground state vibrationally elastic scattering and that is the observation of anti-correlation for the vibrationally inelastic $\mathrm{HCl}(\mathrm{v}=2 \rightarrow 1)$ as well as for the vibrationally elastic $\mathrm{HCl}(\mathrm{v}=2 \rightarrow 2)$ scattering. It appears that the vibrationally inelastic scattering exhibits a stronger anti-correlation than the vibrationally elastic scattering does. This may be induced by steric effects present for vibrational relaxation that are unimportant for vibrationally elastic scattering. A more detailed study of anti-correlation effects in this system would be valuable.

Another important effect on the final translational energy distributions is the temperature of the surface. Here translational energy distributions were measured as a function of surface temperature, between $T_{S}=173$ and $1000 \mathrm{~K}$, for both the vibrationally elastic and inelastic channel.

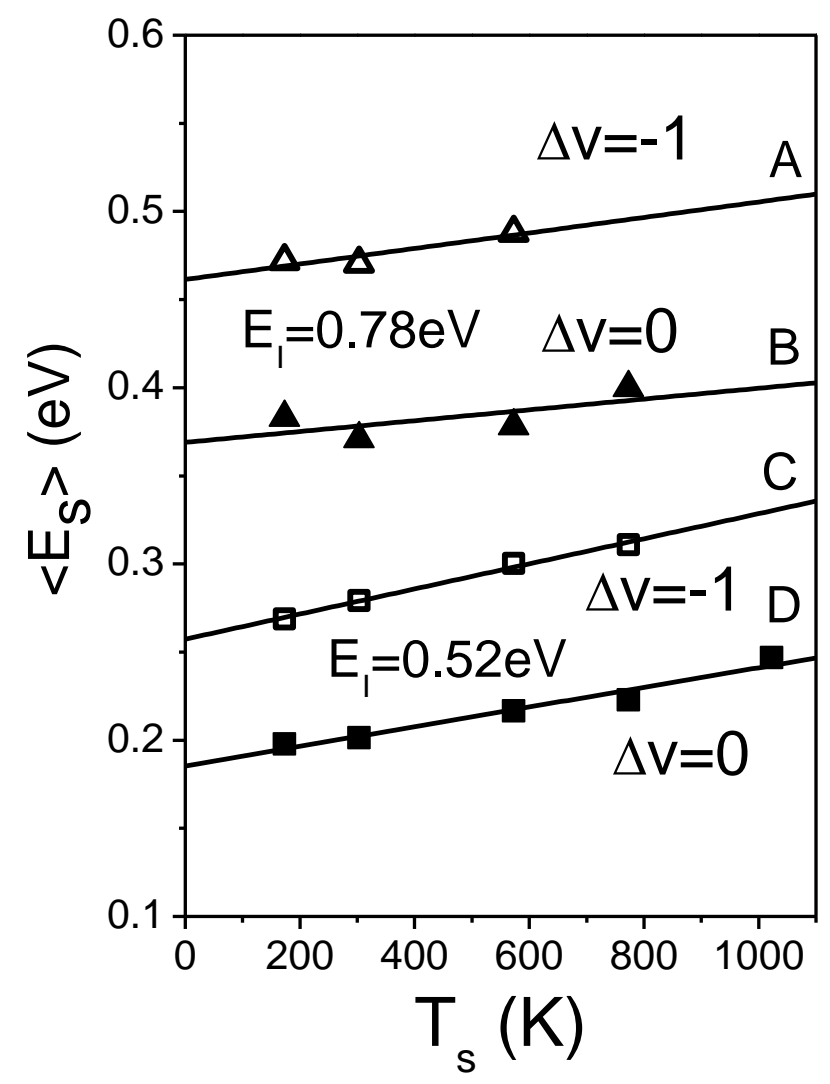

Figure 4.8 Scattered average translational energy as a function of surface temperature for vibrationally elastic, filled symbols, and inelastic, empty symbols, scattering. Triangles represent $\mathrm{E}_{\mathrm{i}}=0.78 \mathrm{eV}$, squares $\mathrm{E}_{\mathrm{i}}=0.52 \mathrm{eV}$. The solid lines are empirical fits to the data.

For both the vibrationally inelastic and elastic scattering, the average translational energy increases with increasing surface temperature. Although it cannot be seen in Fig. 4.8 the translational energy distributions are broadened with increased surface temperature. Both the 
increase of final average translational energy and the broadening of the translational energy distribution are not surprising as the increased thermal motion of the surface atoms leads to a broader spread of microscopic collision conditions. However, the increase in translational energy is not large between $\mathrm{T}_{\mathrm{S}}=173$ and $1000 \mathrm{~K}$ and follows a linear dependence on surface temperature. The temperature dependencies shown in Fig. 4.8, as well as for ground state vibrationally elastic scattering, not shown, can be described by the following equations:

$$
\begin{array}{ll}
\left\langle E_{S}>=7.1_{ \pm 0.43} \times 10^{-5} T_{S}+0.257_{ \pm 0.002}\right. & \mathrm{HCl}(\mathrm{v}=2 \mathrm{~J}=1 \rightarrow \mathrm{v}=1 \mathrm{~J}=5), \mathrm{E}_{\mathrm{i}}=0.52 \mathrm{eV} \\
\left\langle E_{S}>=5.57_{ \pm 0.64} \times 10^{-5} T_{S}+0.185_{ \pm 0.004}\right. & \mathrm{HCl}(\mathrm{v}=2 \mathrm{~J}=1 \rightarrow \mathrm{v}=2 \mathrm{~J}=5), \mathrm{E}_{\mathrm{i}}=0.52 \mathrm{eV} \\
\left\langle E_{S}>=4.497 \times 10^{-5} T_{S}+0.224\right. & \mathrm{HCl}(\mathrm{v}=0 \mathrm{~J}=0 \rightarrow \mathrm{v}=0 \mathrm{~J}=4), \mathrm{E}_{\mathrm{i}}=0.52 \mathrm{eV} \\
\text { and } & \\
<E_{S}>=4.40_{ \pm 1.82} \times 10^{-5} T_{S}+0.461_{ \pm 0.007} & \mathrm{HCl}(\mathrm{v}=2 \mathrm{~J}=1 \rightarrow \mathrm{v}=1 \mathrm{~J}=5), \mathrm{E}_{\mathrm{i}}=0.78 \mathrm{eV} \\
\left\langle E_{S}>=3.07_{ \pm 2.41} \times 10^{-5} T_{S}+0.369_{ \pm 0.012}\right. & \mathrm{HCl}(\mathrm{v}=2 \mathrm{~J}=1 \rightarrow \mathrm{v}=2 \mathrm{~J}=5), \mathrm{E}_{\mathrm{i}}=0.78 \mathrm{eV}
\end{array}
$$

These equations allow for an extrapolation to a zero temperature surface, where we remove the effect from the motion of the surface atoms. At both incidence energies the correction from $T_{S}=300 \mathrm{~K}$ to $T_{S}=0 \mathrm{~K}$ surface temperatures of the translational energy of the scattered molecules is small, $0.02 \mathrm{eV}$. This is coincidently similar in magnitude to the correction for rotational energy $\mathrm{J}=5$ to $\mathrm{J}=1$, but in the opposite direction. These corrections are small and the combination of the two corrections tends to counteract one another. This means the results at $\mathrm{T}_{\mathrm{S}}=300 \mathrm{~K}$ and $\mathrm{J}=5$ do not deviate significantly from what would be expected at $T_{\mathrm{S}}=0 \mathrm{~K}$ surface temperature and $\mathrm{J}=1$ final rotational state, assuming this extrapolation is valid.

\subsection{Coupling of vibrational and translation of a scattered molecule}

Based on preliminary estimates, the $\mathrm{HCl}(\mathrm{v}=2 \rightarrow 2)$ and $\mathrm{HCl}(\mathrm{v}=2 \rightarrow 1)$ channels are of a comparable magnitude under the experimental conditions used here. While no detailed study of the survival probability of $\mathrm{HCl}(\mathrm{v}=2)$ is made, an estimation based on the relative intensities of the REMPI spectra indicate a relatively large vibrational relaxation:

$$
\frac{v=1_{\text {scattered }}}{v=2_{\text {scattered }}}>0.1
$$

It is likely this substantial vibrational relaxation involves coupling to electron hole pairs in the metal. Vibrationallly excited molecules scattering from insulators typically show very small amount of vibrational relaxation, for example $\mathrm{NO}(\mathrm{v}=1)$ scattered from $\mathrm{LiF}(001)$ showed no vibrational relaxation. ${ }^{69,70}$ Perhaps more germane is the study of Korolick et al. who saw $\mathrm{HCl}(\mathrm{v}=2 \rightarrow 1)$ vibrational relaxation, in scattering from $\mathrm{MgO}$, only when trapping desorption was the primary scattering mechanism. ${ }^{71}$ The $\mathrm{HCl} / \mathrm{Au}(111)$ system by comparison shows clear vibrational relaxation from $\mathrm{HCl}(\mathrm{v}=2 \rightarrow 1)$ in a direct scattering process. This indicates that the 
vibrational relaxation pathway here is strongly coupled to electron hole pairs. Also mismatch of energy between phonons of $\mathrm{Au}\left(118 \mathrm{~cm}^{-1}\right)$ and the vibrational energy lost in vibrational relaxation $\mathrm{HCl}(\mathrm{v}=2 \rightarrow 1)\left(3000 \mathrm{~cm}^{-1}\right)$ would mean that the amount of vibrational energy we see transferring to the surface would require the excitation of 12 phonons. Such mulitphonon excitation seems unlikely to be efficient.

Recent unpublished calculations from DH Zhang show the barrier to dissociation on $\mathrm{Au}(111)$ to be comparable to the vibrational energy in $\mathrm{HCl}(\mathrm{v}=2)$. If this is indeed the case then trajectories that sample the reactive transition, but fail to dissociate might undergo vibrational relaxation. This line of reasoning has been previously used to describe the vibrational excitation of $\mathrm{H}_{2}$ on $\mathrm{Cu}(111) .^{72}$

As shown in Fig. 4.6, at $\mathrm{E}_{\mathrm{i}}=0.52 \mathrm{eV}$, the $\mathrm{HCl}(\mathrm{v}=2 \rightarrow 1)$ channel shows a broader and slightly higher translational energy distribution. Similar plots were made for the six additional gas mixtures used in this study.

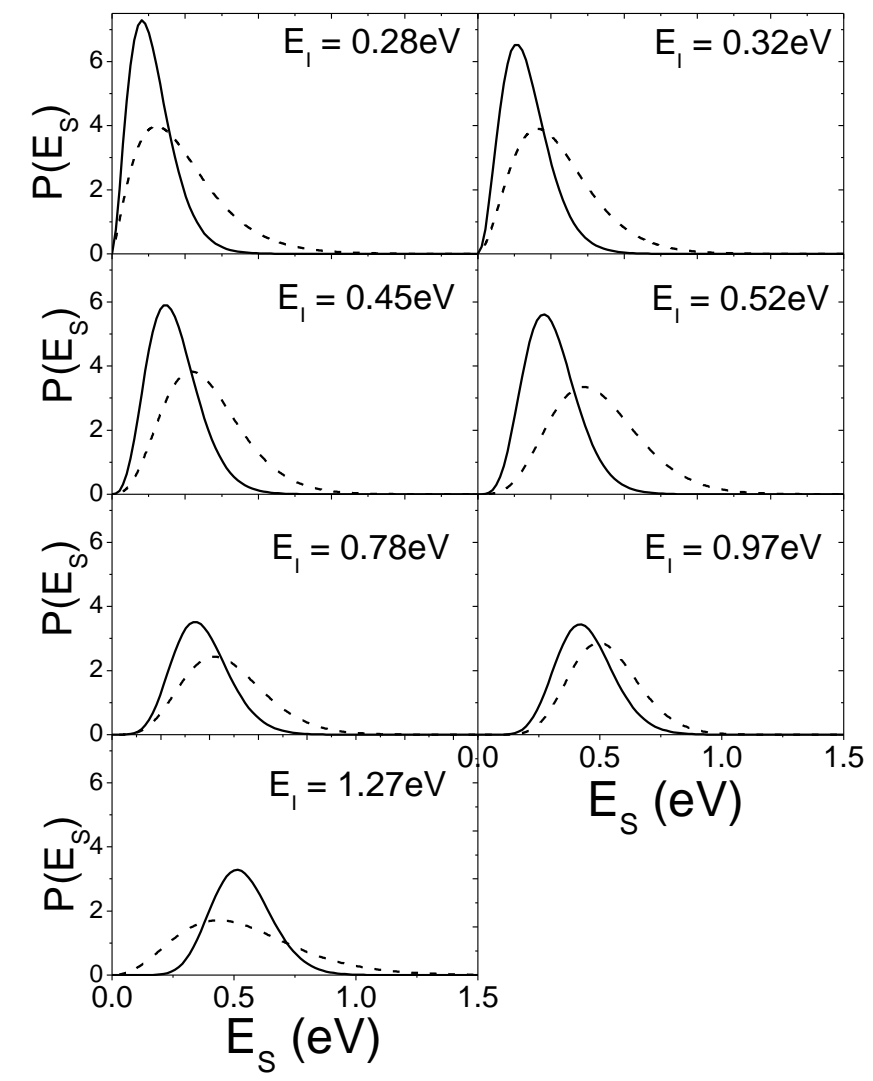

Figure 4.9 Translational energy distributions for vibrationally elastic $\mathrm{HCl}(\mathrm{v}=2 \rightarrow 2)$, solid lines, and inelastic $\mathrm{HCl}(\mathrm{v}=2 \rightarrow 1)$, dashed lines, scattering at several different translational incidence energies at $\mathrm{T}_{\mathrm{S}}=300 \mathrm{~K}^{50}$ 
For both vibrationally elastic and inelastic channels, the translational energy distribution increases with increasing incidence energy and broadens. Quantitative parameters describing the velocity distributions are listed in Table 4.2 and 4.3.

Previous reports for non-adiabatic vibrational energy transfer at surfaces have indicated that the kinetic energy change of the scattered molecules does not depend on $\Delta \mathrm{v} .{ }^{8,10}$ As mentioned in the background section, NO in high vibrational states $(v=15)$ has been scattered from Au surfaces losing large amounts of vibrational energy $(\sim 1.5 \mathrm{eV})$, but no noticeable translational energy change was seen. ${ }^{10}$ Previous studies of $\mathrm{NO}(\mathrm{v}=0 \rightarrow 1)$ vibrational excitation also did not show a striking difference in the translational energy distribution between elastic and inelastic scattering. ${ }^{29}$ This work also showed a zero-threshold for vibrational excitation dependence on incidence energy of translation, compared to a clear threshold for mechanical excitation. ${ }^{30}$ These results led to the idea that translational energy, although important in the coupling of molecular vibration to surface electrons, was itself a spectator. That is to say, V-T coupling is very weak. Now compare this spectator view to the results shown for the vibrational relaxation of $\mathrm{HCl}$ shown here. At all incidence energies the vibrationally inelastic channel has a translational energy distribution that is slightly broader and more energetic than the vibrationally elastic channel. This is seen in Fig. 4.9. In Fig. 4.10 the average energy for vibrationally inelastic and elastic scattering as a function of incidence energy of translation is plotted.

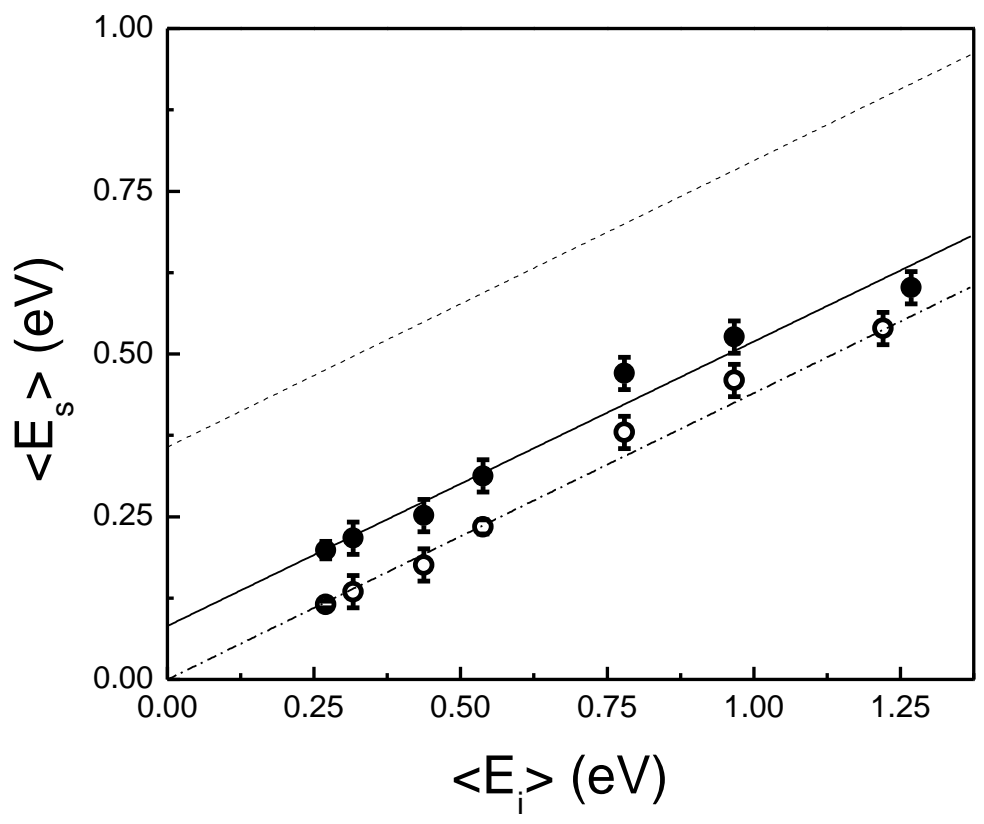

Figure 4.10 Final average translational energy of the vibrationally elastic, empty circles, and inelastic, filled circles, plotted as a function of incidence energy of translation. The dot dashed line represents a linear fit $\left\langle E_{s}\right\rangle=0.44\left\langle E_{i}\right\rangle$ for the vibrationally elastic case. The solid line represents $\left\langle E_{s}\right\rangle=0.44\left\langle E_{i}\right\rangle+0.0821 \mathrm{eV}$ for inelastic scattering. The dashed line represents the expected inelastic scattering if all released vibrational energy appeared as translation. ${ }^{50}$ 
It is clear from Fig. 4.10 that the spectator view of translational energy in vibrational energy change is not fully observed. Indeed at all incidence energies, $26 \%$ of the vibrational energy is coupled to translation. This means that the naïve "spectator" view of the translation of the molecule is not fully correct. Possible mechanisms for this coupling of molecular vibration to translation will be discussed.

An interesting comparison is to $\mathrm{H}_{2}$ scattering from $\mathrm{Pd}^{73}$ Here $\mathrm{H}_{2}$ in $\mathrm{v}=1 \mathrm{~J}=1$ was shown to relax with a high probability to $\mathrm{v}=0 \mathrm{~J}=\mathrm{J}^{\prime}$. The velocity of the molecules was measured in a way similar to the $\mathrm{HCl}$ translational energy studied here. Sitz et al. argued that the large relaxation probability was indicative of electronically non-adiabatic vibrational relaxation. However a large fraction of the vibrational energy of the scattered $\mathrm{H}_{2}$ coupled to the translation of the molecule. In fact more energy from vibration coupled to translation of the molecule than coupled to the surface. ${ }^{73}$ This indicates that the coupling of molecular vibration to translation is not limited to the $\mathrm{HCl} / \mathrm{Au}(111)$ system.

One possible explanation is that the difference arises due to steric effects. These can be treated in an analogous fashion to previous work by Kimman who showed that total energy transferred to the system decreased with increased scattered rotational state. ${ }^{68}$ The reasoning was that molecules that scattered from the surface in an orientation that was favorable for rotational excitation were not oriented properly for coupling translational energy to the surface. Kroes et al. showed that $\mathrm{H}_{2}$ dissociation occurs at different surface sites depending on initial quantum state. $^{74-76}$ The extension of this idea to the present work suggests that molecules in orientations more likely undergo vibrational relaxation are also more likely to be in an orientation that retains more of its translational energy upon scattering.

Two aspects of these results make this idea seem unlikely. First, in Fig. 4.6 and 4.9 it can be seen that, especially at low incidence energies of translation, the vibrationally inelastic channel has a non-negligible fraction of molecules with energies that exceed that of the incident beam. Since the scattered translational energy exceeds that of the incident molecules, it would seem that vibrational energy is coupled directly to translation and the difference between vibrationally elastic and inelastic is not caused by molecular orientation effects.

Additionally the difference between vibrationally elastic and inelastic translational energy is independent on incidence energy of translation, $\sim 0.08 \mathrm{eV}$. If molecular orientation was indeed the driving force behind the translational energy difference seen here, one might expect that the slope of the elastic and inelastic translational energy dependence, shown in Fig. 4.10, would be different and for both of them to extrapolate to the origin. However, in this work the vibrationally inelastic case does not extrapolate to the origin. These two factors indicate that the orientation of the $\mathrm{HCl}$ when scattering from the $\mathrm{Au}(111)$ surface is not the predominant cause of the translational energy difference between the vibrationally elastic and inelastic scattering channels. 
Another possibility for the $\mathrm{T}-\mathrm{V}$ coupling is that some molecules undergo vibrational relaxation via an electron mediated process while others undergo a mechanical relaxation. An attempt to fit the scattered translational distributions with a bimodal distribution accounting for these two mechanisms was undertaken.

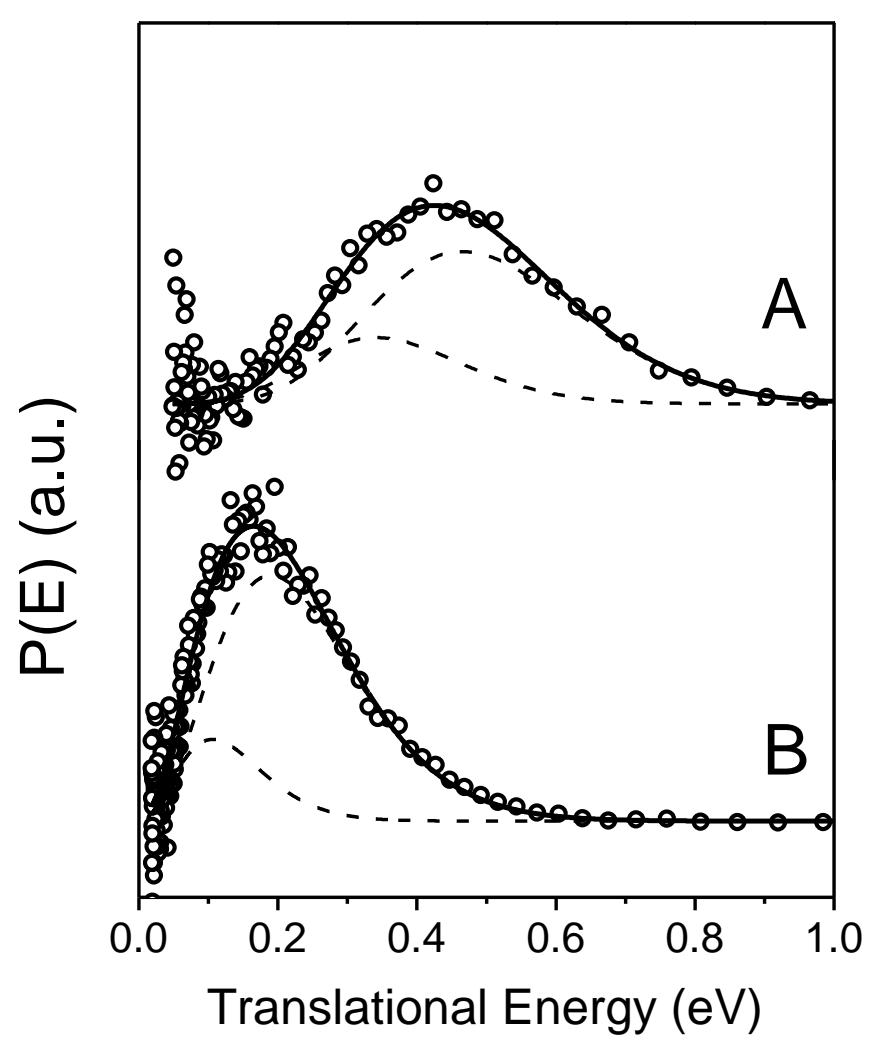

Figure 4.11 Fitting of scattered vibrationally inelastic $\mathrm{HCl}(\mathrm{v}=2 \rightarrow 1)$ translational energy distributions, at two incidence energies 0.78 , A, and $0.32 \mathrm{eV}, \mathrm{B}$, of $\mathrm{v}=1 \mathrm{~J}=5$ at $300 \mathrm{~K}$ surface temperature with two components. The open circles represent experimental measurements, the solid lines fits as described in Appendix I. The dashed lines represent the fitting procedure for a bimodal distribution described in text.

The idea of two competeing mechanisms leading to the increased translational energy seen in the vibrational relaxation channel is a bit unlikely however for two reasons. First no hint of bimodal features is seen at any of the incidence energies, see Fig. 4.5. In addition when a bimodal fitting procedure is applied, see below for details, no evidence of a fast peak with the energy expected in purely mechanical vibrational relaxation is seen.

To demonstrate this I fit data like that in Fig. 4.9 to bimodal translational energy distributions described by: 


$$
P(E)=A_{1} \frac{E}{m^{2}} e^{-\frac{2}{m \alpha_{1}^{2}}\left(\sqrt{E}-\sqrt{E_{01}}\right)^{2}}+A_{2} \frac{E}{m^{2}} e^{-\frac{2}{m \alpha_{2}^{2}}\left(\sqrt{E}-\sqrt{E_{02}}\right)^{2}}
$$

where $\alpha_{2}$ and $E_{02}$ are obtained from the fit of vibrationally elastic channels and are kept constant. The relative amplitudes of two components $A_{1}$ and $A_{2}$ as well as the values defining the width and the position of the faster component $\alpha_{1}$ and $E_{01}$ are free parameters. Figure 4.11 displays examples from this analysis for two incidence energies. This bimodal fitting procedure results in a fast component that exhibits a mean scattered translational energy only $\sim 0.026 \mathrm{eV}$ higher than the values reported in Fig. 4.10. This analysis makes us doubt that the molecules undergo two distinctly different mechanisms of vibrational relaxation.

Two more possibilities exist which may lead to this difference in translational energy. One is that in the electronically non-adiabatic vibrational energy transfer a transient ion is formed. If the transient ion lives long enough, its attractive image charge would accelerate the molecule toward the surface. The molecule would then undergo translational energy transfer in the same manner as in the vibrationally elastic case, but at a higher initial translational energy. It would then release the electron back to the surface and fly back as a neutral molecule. One other possibility is that the translation of the molecule is only partially a spectator in vibrational relaxation and vibrational energy couples directly to both the translation of the molecule and the electrons in the surface. It is at present not clear which of these mechanisms is a better description of the results shown here. Comparisons to high level theoretical simulations would be very helpful.

Scattered vibrationally excited molecules were shown to undergo vibrational relaxation at the surface in a direct scattering mechanism. The view of translation as a spectator, in electronically non-adiabatic vibrational energy transfer, was seen to be only partially true. $26 \%$ of the molecular vibrational energy lost was transferred to translation, and this was independent of the incidence energy of translation of the molecules. Several mechanisms for this coupling were postulated and their likelihood discussed.

\subsection{Vibrationally elastic scattering:}

State-to-state experiments of translational energy transfer often utilize optical pumping of the incident molecules to a vibrationally excited state prior to scattering from the surface. ${ }^{10,70,77,78}$ Laser preparation of a single quantum state occurs in a very small volume. This allows for a narrow distribution of collision times, a fraction of a microsecond, which is significantly shorter than molecular beams prepared by mechanical choppers or shutters. The distribution of the arrival time of the incident beam on the surface typically limits the resolution of the TOF measurements used to calculate the translational energy of the outgoing molecules. This is exactly the technique used above for $\mathrm{HCl}(\mathrm{v}=2)$ scattering. However it would be advantageous to 
have the same $\mathrm{TOF}$ resolution seen in $\mathrm{HCl}(\mathrm{v}=2)$ elastic scattering for the vibrational ground state, $\mathrm{HCl}(\mathrm{v}=0)$. The technique utilized to make accurate translational energy measurements for $\mathrm{HCl}(\mathrm{v}=0)$ is now described.

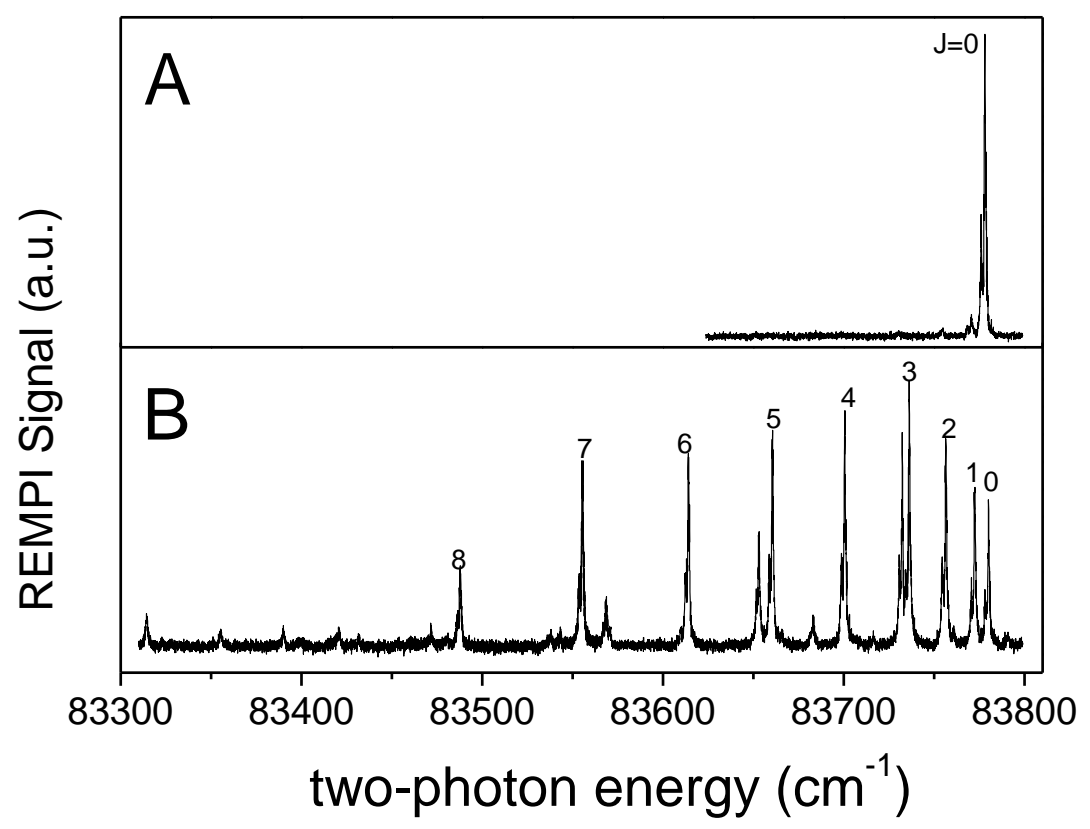

Figure 4.12 Comparison of REMPI spectra of incident, A, and scattered, B, beams. The lines are consist of transitions in the $\mathrm{Q}$ branch of the $\mathrm{E}^{1} \Sigma^{+}\left(v^{\prime}=0\right) \leftarrow \mathrm{X}^{1} \Sigma^{+}\left(v^{\prime \prime}=0\right)$ band. The numbers represent the rotational quantum numbers in the ground state. This spectra was recorded at $\mathrm{E}_{\mathrm{i}}=0.52 \mathrm{eV}$ and $\mathrm{T}_{\mathrm{S}}=300 \mathrm{~K}$.

The dominant process in ground state $\mathrm{HCl}$ scattering from the surface is rotational excitation. As the incident molecular beam is rotationally cold, only the lowest $\mathbf{J}$ states have significant population. For all scattering conditions utilized here significant rotational excitation takes place upon scattering from the surface. Tuning the IR pump laser allows for optical vibrational excitation of these excited rotational states $\mathrm{HCl}\left(\mathrm{v}=0, \mathrm{~J}=\mathrm{J}^{\prime} \rightarrow \mathrm{v}=2, \mathrm{~J}=\mathrm{J}^{\prime} \pm 1\right)$. In this work, the $\mathrm{R}$ branch of the overtone excitation was utilized. $\mathrm{J}^{\prime}=4$ was typically used, although in theory any $\mathrm{J}$ state can be used. These excited molecules are created in a small space allowing for a welldefined spatial origin. These excited molecules then travel a defined distance to the REMPI laser where they are state selectively ionized and detected. By varying the timing between the two lasers the optically pumped "overtone tagged" $\mathrm{HCl}(\mathrm{v}=2)$ molecules arrival time is recorded. This allows for the calculation of the velocity and consequently translational energy distributions of vibrationally elastic $\mathrm{HCl}(\mathrm{v}=0 \rightarrow 0)$ scattering. 


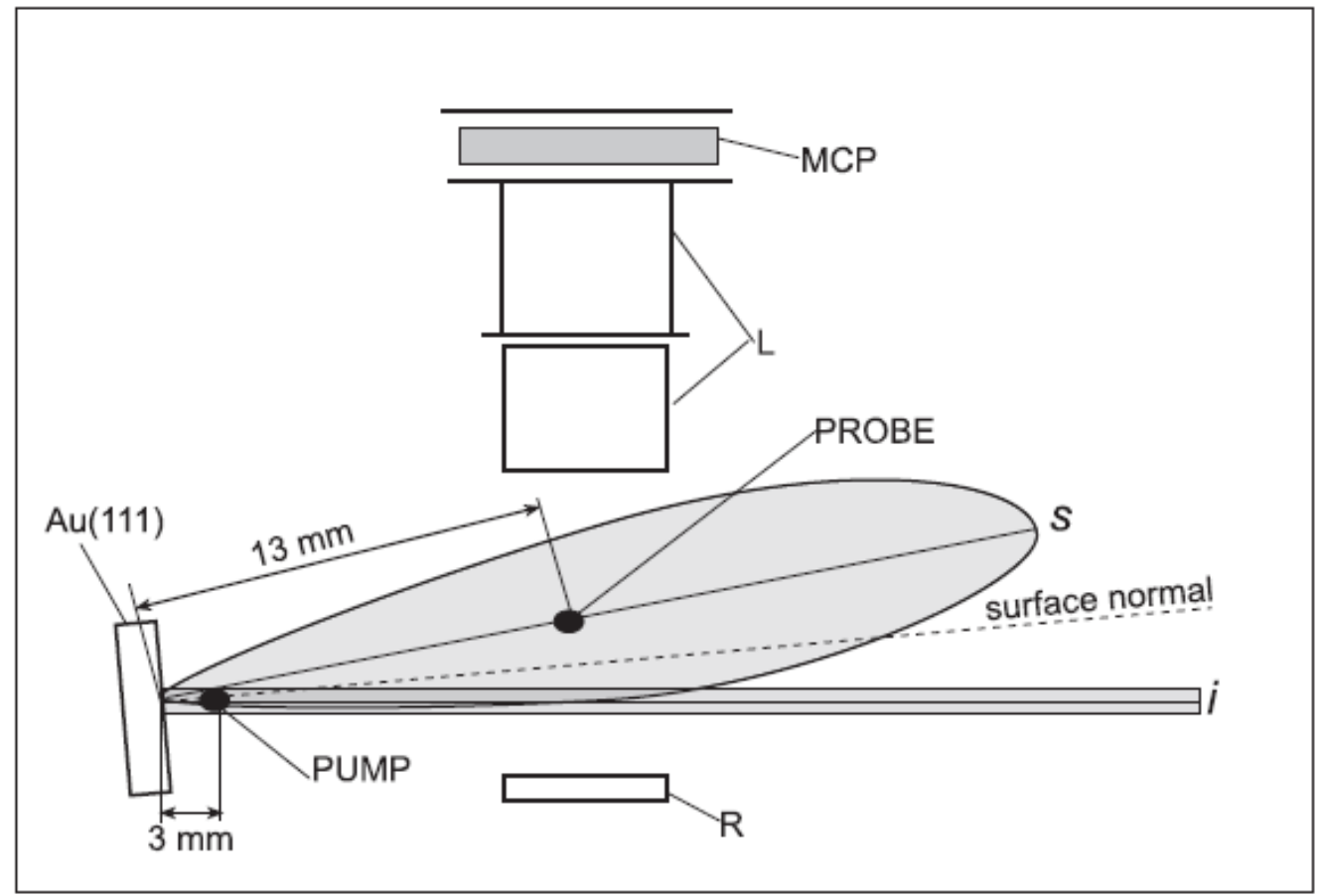

Figure 4.13 Geometry used to measure $\operatorname{HCl}(\mathrm{v}=0)$ scattering. The molecules undergo specular scattering. The pump laser is raised to the specular angle $3 \mathrm{~mm}$ from the surface. The probe laser sits $13 \mathrm{~mm}$ from the surface. Altering the time of the firing of the lasers relative to each other allows for TOF measurements to be made.

The experimental set-up for the measurement of translational energy transfer of $\mathrm{HCl}(\mathrm{v}=0 \rightarrow 0)$ scattering is very similar to that previously described for $\mathrm{HCl}(\mathrm{v}=2 \rightarrow 2)$. Here the pump beam is close to the surface, but raised slightly to lie at the specular angle of scattering, as shown 4.13. 


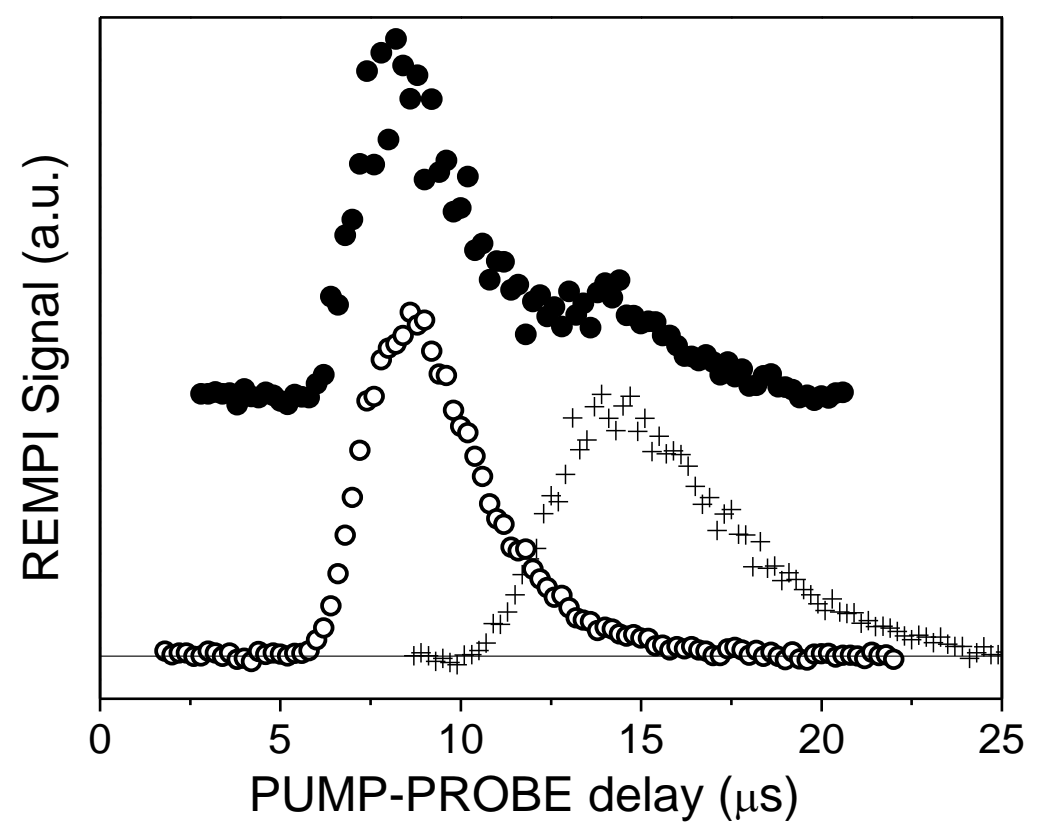

Figure 4.14 Discriminating between ground $\mathrm{HCl}(\mathrm{v}=0 \rightarrow 0)$ and excited state $\mathrm{HCl}(\mathrm{v}=2 \rightarrow 2)$ scattering by altering the pumping and probing wavelengths. Filled circles represent pumping from $\mathrm{HCl}(\mathrm{v}=0 \mathrm{~J}=0 \rightarrow \mathrm{v}=2 \mathrm{~J}=1)$ and probing $\mathrm{HCl}(\mathrm{v}=2 \mathrm{~J}=1)$. A bimodal TOF is observed, where the early peak and late peak representing overtone pumping before and after collision with the surface. Empty circles represent pumping from $\mathrm{HCl}(\mathrm{v}=0 \mathrm{~J}=4 \rightarrow \mathrm{v}=2 \mathrm{~J}=5)$ and probing $\mathrm{HCl}(\mathrm{v}=2 \mathrm{~J}=5)$ representing $\mathrm{HCl}(\mathrm{v}=0 \rightarrow 0)$ scattering. Crosses represent pumping from $\mathrm{HCl}(\mathrm{v}=0 \mathrm{~J}=0 \rightarrow \mathrm{v}=2$ $\mathrm{J}=1)$ and probing $\mathrm{HCl}(\mathrm{v}=2 \mathrm{~J}=5)$, which represents $\mathrm{HCl}(\mathrm{v}=2 \rightarrow 2)$ scattering from the surface as rotational excitation is required at the surface in order for signal to be seen at a rotational state higher than that excited by the IR laser. ${ }^{79}$

Measurements of $\mathrm{HCl}(\mathrm{v}=0 \rightarrow 0)$ scattering require pumping of the molecules after the scattering event. Since the rotationally cold molecules in the incident beam undergo extensive rotational excitation upon scattering from the surface, the molecules pumped from excited rotational states, for example $\mathrm{HCl}(\mathrm{v}=0 \mathrm{~J}=4 \rightarrow \mathrm{v}=2 \mathrm{~J}=5$ ) (empty circles in Fig. 4.14), must occur after the scattering event. Molecules which are pumped from the ground rotational state $\mathrm{HCl}(\mathrm{v}=0 \mathrm{~J}=0 \rightarrow \mathrm{v}=2 \mathrm{~J}=1)$ and probed in $\mathrm{HCl}(\mathrm{v}=2 \mathrm{~J}=5)$ can only arise from molecules that are vibrationally excited before interacting with the surface and are rotationally excited in the scattering process (crosses in Fig. 4.14 ). This allows an independent comparison of molecules with the incidence quantum state of $\mathrm{HCl}(\mathrm{v}=0 \mathrm{~J}=0)$ and $\mathrm{HCl}(\mathrm{v}=2 \mathrm{~J}=1)$. Note that the difference in arrival time shown in Fig. 4.14 arises primarily from the different flight distances for molecules excited before the surface, compared to those that are excited after the surface. When pumping $\mathrm{HCl}(\mathrm{v}=0 \mathrm{~J}=0 \rightarrow \mathrm{v}=2 \mathrm{~J}=1)$ and probing $\mathrm{HCl}(\mathrm{v}=1 \mathrm{~J}=1)$, molecules pumped before and after scattering are seen concurrently(filled circles in Fig. 4.14). Here we show that with careful consideration of the quantum state pumped and probed, accurate TOF measurements can be made for molecules with different initial vibrational quantum states. 


\subsection{Comparison of translational inelasticity for $\mathrm{HCl} v=2$ and $\mathrm{v}=0$ vibrationally elastic scattering:}

Using the techniques described above, I measured translational energy distributions at seven incidence energies for $\mathrm{HCl}(\mathrm{v}=2 \rightarrow 2)$ and $\mathrm{HCl}(\mathrm{v}=0 \rightarrow 0)$ vibrationally elastic scattering. A comparison of the translational energy distributions is shown in Fig. 4.15.

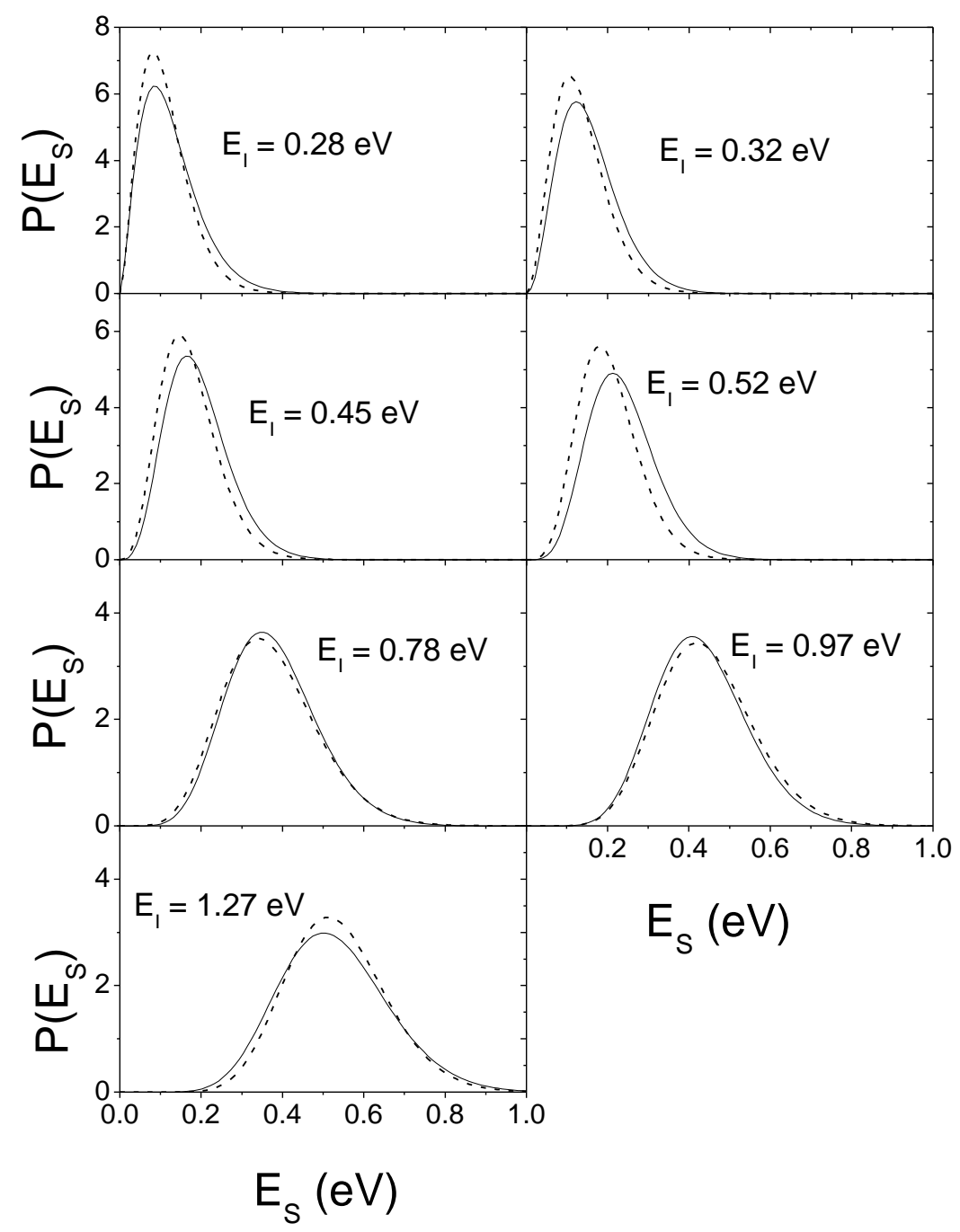

Figure 4.15 Derived translational energy distributions for vibrationally elastic scattering of $\mathrm{HCl}$ scattering from $\mathrm{Au}(111)$ at various incidence energies for $\mathrm{T}_{\mathrm{S}}=300 \mathrm{~K}$. Solid lines represent $\mathrm{HCl}(\mathrm{v}=0 \rightarrow 0, \mathrm{~J}=0 \rightarrow 4)$, dashed lines $\mathrm{HCl}(\mathrm{v}=2 \rightarrow 2, \mathrm{~J}=1 \rightarrow 5) .^{79}$

It is clear from Fig. 4.15 that there is little difference between the translational energy distributions of $\mathrm{HCl}(\mathrm{v}=0 \rightarrow 0)$ and $\mathrm{HCl}(\mathrm{v}=2 \rightarrow 2)$ scattering. In most cases both the peak of the probability distribution and its width are almost identical. Despite the fact that these experiments are similar there are several experimental conditions that differ, such as background, signal-to- 
noise, and flight distances. This indicates that the methods used here to measure translational energies are likely free of large systematic errors.

Figure 4.16 shows the average value of the translational energy for the vibrationally elastic scattering channels as a function of incidence energy of translation. Again as seen in Fig. 4.15 there is no discernable difference in the $\mathrm{HCl}(\mathrm{v}=0 \rightarrow 0)$ and $\mathrm{HCl}(\mathrm{v}=2 \rightarrow 2)$ cases.

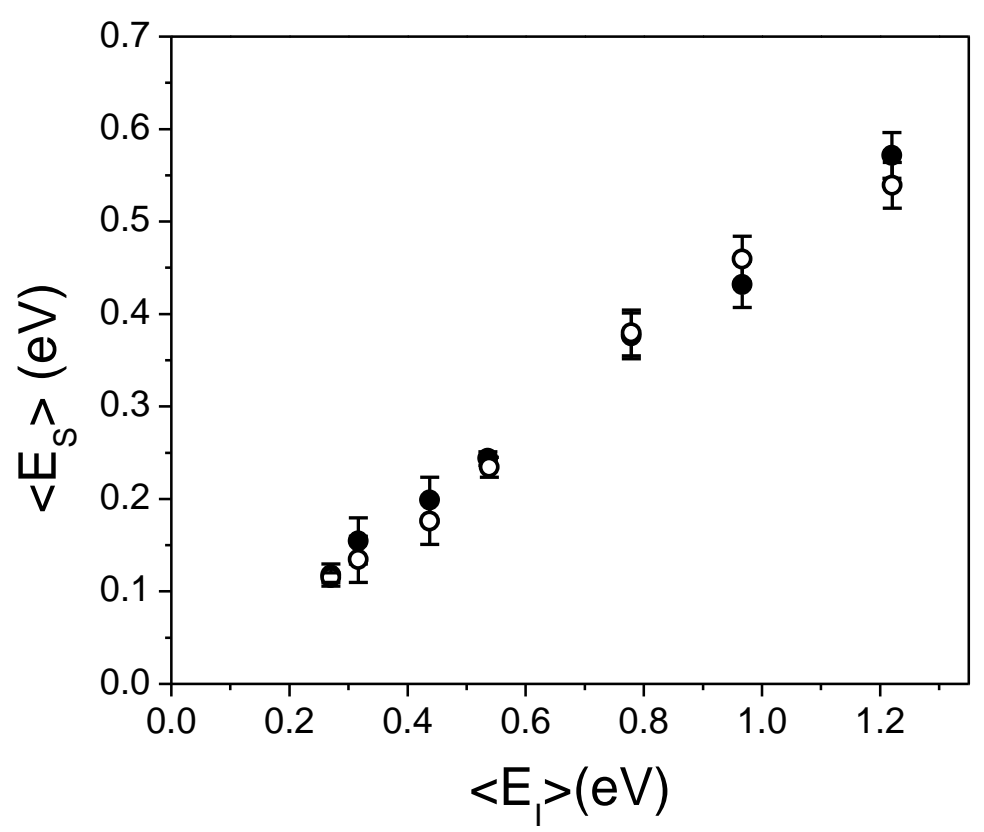

Figure 4.16 Dependence of the average translational energy of scattered molecules on the incidence energy of translation at $\mathrm{T}_{\mathrm{S}}=300 \mathrm{~K}$. Filled circles represent $\mathrm{HCl}(\mathrm{v}=0 \rightarrow 0, \mathrm{~J}=0 \rightarrow 4)$, empty circles $(\mathrm{v}=2 \rightarrow 2, \mathrm{~J}=1 \rightarrow 5)$ The error bars displayed in the figure are based on the combined statistical uncertainty associated with the mean energy evaluation as well as the systematic error associated with uncertainty of the distances between pump and probe laser beams and the gold crystal.

Results in Fig. 4.16 show that that under all conditions a large fraction of the translational energy is transferred to the surface. The transfer of translational energy is independent of the initial vibrational state. A more detailed discussion of the translational energy transfer in vibrationally elastic scattering will involve only the $\mathrm{HCl}(\mathrm{v}=2 \rightarrow 2)$ channel. Since there is not noticeable difference for $\mathrm{HCl}(\mathrm{v}=0 \rightarrow 0)$ and $\mathrm{HCl}(\mathrm{v}=2 \rightarrow 2)$ the discussion is valid for both cases.

\subsection{Comparison to previous $\mathrm{HCl}$ translational energy transfer}

We may compare to a previous report of the translational inelasticity of $\mathrm{HCl}$ scattering from $\mathrm{Au}(111) .{ }^{80}$ Although in the previous study the reported error bars were large, those measurements suggested approximate translational elasticity, $\left\langle\mathrm{E}_{\mathrm{s}}\right\rangle=1.0 \pm 0.2\left\langle\mathrm{E}_{\mathrm{i}}\right\rangle$ in apparent contradiction to the present work. Significant differences between the experimental conditions are the likely explanation for this contradiction. First, the resolution of the TOF experiments differs widely, and is much higher in the present work. Second, the geometries of the two 
experiments are quite different. In the previous work a detection orientation of $\theta_{\mathrm{i}}=\theta_{\mathrm{f}}=45^{\circ}$ was employed, and this biased the observations toward translational elasticity, since as translational energy is lost preferentially from the normal component of velocity, translationally inelastic scattering leads to non-specular scattering.

The improved TOF resolution in the present study arises mainly from reducing the uncertainty in the "start time" from the surface in comparison to the earlier experiments. In my experiment, I "tag" molecules using a focused IR laser beam with diameter $\mathrm{d}_{\mathrm{IR}}$. These molecules then fly a distance of $10 \mathrm{~mm}, 1_{\text {flight }}$, and are detected by a tightly focused UV REMPI laser. The distribution of flight times resulting from the spread in flight lengths is simply $\mathrm{d}_{\mathrm{IR}} / /_{\text {flight }} \sim 0.1 \mathrm{~mm} / 10 \mathrm{~mm}=$ 0.01. Since the velocity spread in the incident beam is larger than this $(\sim 5 \%)$, the resolving power of my experiment is determined mainly by the velocity spread of the incident beam. The energy shift we see $(\sim 50 \%)$ is well within the resolving power of the experiment.

In the previous work the molecular beam is mechanically chopped prior to encountering the surface. ${ }^{80}$ The arrival time distribution at the surface is a convolution of the shutter function of the chopper and dispersion in times resulting from the velocity spread of the molecular beam projected over the distance traveled from the chopper to the surface. As this distance was large, $700 \mathrm{~mm}$, this second factor is very important. At the incidence energies used to carry out this previous study, the width of the distributions characterizing the arrival time at the surface ranges from 20 to $50 \mu \mathrm{s}$. Thus, precise knowledge of the "start time" from the surface is limited. To show the resolution limitations of the previous work, we will estimate the shift in the flight time assuming the translational inelasticity measured here. How large a shift in flight time of the previous experiment would be expected assuming the energy distributions of the results of the present work? In making this estimate, we have to take into account that my experiment involves near normal incidence $\theta_{\mathrm{i}}=\theta_{\mathrm{f}}=3^{\circ}$, while the previous study used a geometry with $\theta_{\mathrm{i}}=\theta_{\mathrm{f}}=45^{\circ}$. Since parallel momentum is approximately conserved, in scattering from the low index planes of a metal surface, half of the incidence translational energy - that which is associated with parallel motion to the surface - is retained in the surface collision. Taking this into account, the time shift expected from the inelasticity of the molecule surface collision would be 20 to $50 \mu$ s, roughly equal to the resolving power of the experiment, as shown in Fig. 4.17. This argument suggests that the resolving power of the previous experiment is perhaps not sufficient to observe the translational inelasticity reported here. 


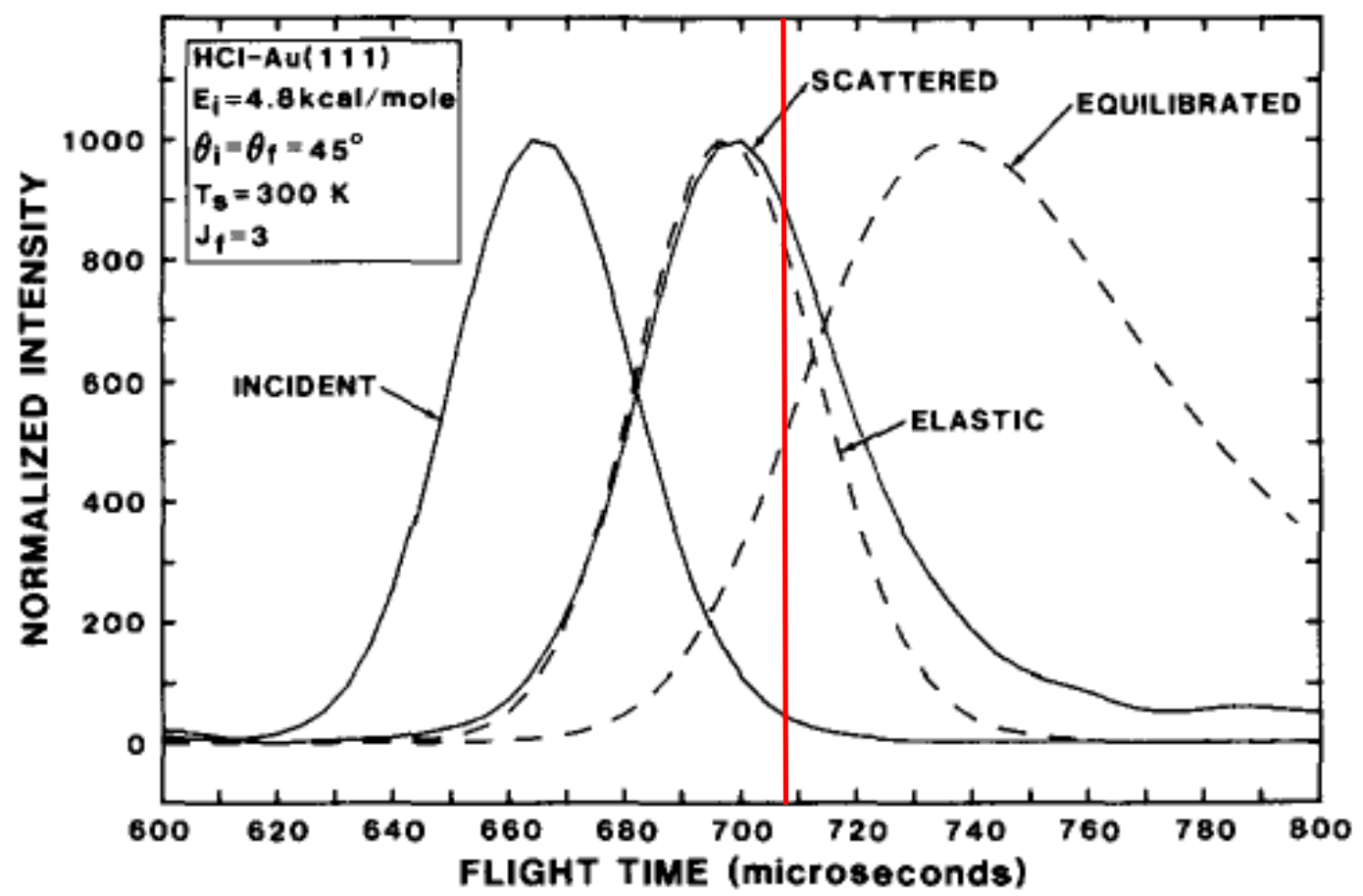

Figure 4.17 REMPI intensity of incident and scattered time of flight distributions from a previous study of $\mathrm{HCl}(\mathrm{v}=0 \rightarrow 0)$ scattered from $\mathrm{Au}(111) \cdot{ }^{80} \mathrm{The}$ incident beam is $\mathrm{HCl}(\mathrm{v}=0 \mathrm{~J}=0)$ and the scattered is $\mathrm{HCl}(\mathrm{v}=0 \mathrm{~J}=3)$. One of the dashed lines corresponds to translationally elastic scattering, the other to a TOF where the molecules have undergone complete thermalization with the surface. The red line indicates the small shift in the peak of the scattered TOF expected if the scattered molecules showed the translational inelasticity measured in this work.

The other experimental factor that may have led the previous observation of translational inelasticity is the detection geometry. Using $\theta_{\mathrm{i}}=\theta_{\mathrm{f}}=45^{\circ}$ detection geometry and bearing in mind that parallel momentum is approximately conserved, the most probable scattering events measured by me, where 0.45 of the energy associated with the motion perpendicular to the surface, is retained, cannot be observed. It can be calculated that if 0.45 of kinetic energy perpendicular to the surface is retained and all the energy associated with motion parallel to the surface is retained, the most likely outgoing scattering angle is $\theta_{\mathrm{f}}=56^{\circ}$ with respect to the surface normal, when $\theta_{\mathrm{i}}=45^{\circ}$. Indeed, use of the " $\theta_{\mathrm{i}}=\theta_{\mathrm{f}}=45^{\circ}$ " detection geometry biases the observation in favor of scattering that is approximately translationally elastic.

\subsection{Comparison of $v=2$ elastic scattering to the Baule model:}

As mentioned earlier, the vibrationally elastic scattering of $\mathrm{HCl}$ from $\mathrm{Au}(111)$ transfers a significant fraction of the translational energy of the molecules to the surface. As was shown in Fig. 4.16, the molecules transfer a constant fraction of the incidence energy of translation, 56\%, to the surface, over the entire range of incidence energies shown here. Neither the surface temperature or final rotational state strongly affected the final translational energy and what small effect they had was largely negated by the fact they were roughly of equal magnitude, but 
in opposite directions. The analysis presented here is not thought to not vary dramatically from the zero temperature zero rotation limit.

One might wonder if this fraction of translational energy lost to the surface, $\left\langle E_{\mathrm{s}}\right\rangle=0.44\left\langle\mathrm{E}_{\mathrm{i}}\right\rangle$, can be explained by a simple mechanical picture, where the translational energy of the $\mathrm{HCl}$ is transferred to motion of the atoms in the surface. To understand this, a simple model which represents the impulsive binary collision limit, often and consequently here referred to as the Baule limit. ${ }^{62}$ Here the collision of the $\mathrm{HCl}$ molecule with the $\mathrm{Au}(111)$ surface happens in a way that the interactions between the $\mathrm{Au}$ atoms are ignored, at least in the time scale of the interaction. The interaction is further approximated as a zero impact parameter binary collision between a spherical projectile, representing the $\mathrm{HCl}$, and a stationary sphere representing the $\mathrm{Au}$ surface. Head on collisions with zero impact parameter transfer the greatest amount of translational energy from the $\mathrm{HCl}$ to the $\mathrm{Au}$ atom. This analysis is equivalent to the hard cube model, with the effective mass of the cube set to that of a single Au atom.

From linear momentum and energy conservation in the collision pair we can derive the fraction of energy retained in the system.

$$
\frac{E_{s}}{E_{i}}=\frac{\left(m_{1}-m_{2}\right)^{2}}{\left(m_{1}+m_{2}\right)^{2}}
$$

For $\mathrm{HCl}$ and $\mathrm{Au}$ collisions, $\mathrm{m}_{1}=36$ and $\mathrm{m}_{2}=196$, leading to $\left\langle\mathrm{E}_{\mathrm{s}}\right\rangle=0.475\left\langle\mathrm{E}_{\mathrm{i}}\right\rangle$, which compares well to the experimentally measured value of $\left\langle\mathrm{E}_{\mathrm{s}}\right\rangle=0.44\left\langle\mathrm{E}_{\mathrm{i}}\right\rangle$. 


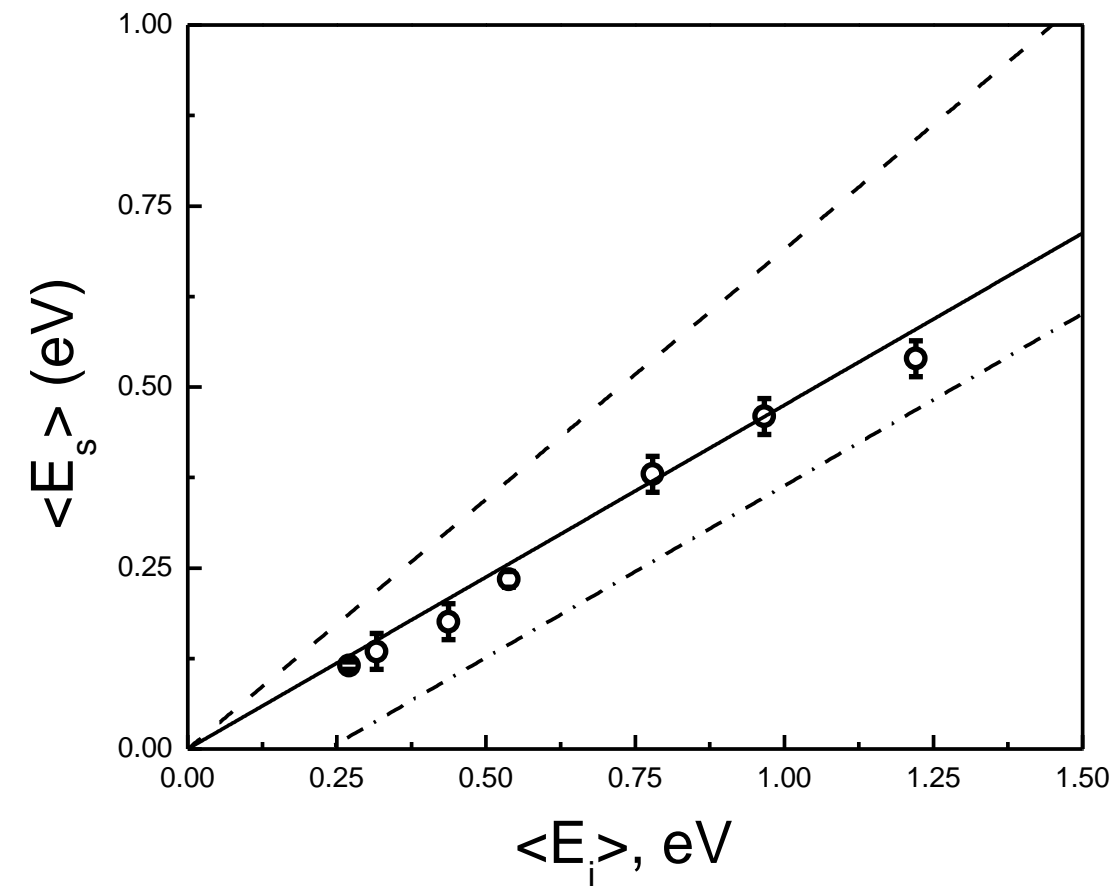

Figure 4.18 Comparison to the binary-collision limit. Final translational energy for scattered $\mathrm{HCl}$ : vibrationally elastic $\mathrm{HCl}(\mathrm{v}=2 \rightarrow 2, \mathrm{~J}=1 \rightarrow 5)$, open circles at $\mathrm{T}_{\mathrm{S}}=300 \mathrm{~K}$. The solid line shows the translational recoil expected from a zero impact-parameter elastic collision of a $\mathrm{HCl}$ molecule with an isolated $\mathrm{Au}$ atom. The dashed line shows results expected by doubling the effective mass of the Au atom. The dashed-dot line is the attractive Baule limit, where the attractive well is treated as a step function of $0.23 \mathrm{eV}$.

Figure 4.18 shows the $\mathrm{HCl}(\mathrm{v}=2 \rightarrow 2)$ vibrationally elastic scattering compared to several different versions of the Baule Model. The solid line is the Baule model with the mass of the Au sphere mass set to one Au atom. The dashed line is the Baule model with the Au sphere mass set to two $\mathrm{Au}$ atoms. Within the Baule model, a larger than predicted translational energy transfer can take place due to the acceleration of the $\mathrm{HCl}$ by the attractive part of the interaction potential. The attractive Baule limit was implemented by approximating the potential as a step function. This tends to overestimate the effect of the well compared to the more accurate Morse function. ${ }^{81}$ However in such a simplified model the step function should serve appropriately to estimate the lower limit of translational energy transfer. This, called the attractive Baule limit, is indicated in Fig. 4.18 by the dot-dashed line. Here an attractive well depth of $0.23 \mathrm{eV}$ was used, based on the orientation averaged binding energy reported by Lykke and Kay. ${ }^{80}$ The attractive Baule limit shows more translational energy transfer than the average value of the translational inelasticity obtained from experiment.

Previous work from several authors has shown that often the average translational energy transfer to the surface is less than that described by the Baule limit. Some of these previous translational energy measurements are shown in Table 4.4. Although there is a fair amount of 
previous work, comparisons are difficult as often the experiments are carried out at non-normal incidence, often $\theta_{\mathrm{i}}=45^{\circ}$. For scattering from close packed surfaces the transfer of the momentum perpendicular to the surface is often much larger than that parallel to the surface. ${ }^{29,82,83}$ In the hard cube model the parallel momentum is obviously conserved, so experiments taking place at non-normal incidence would have artificially low values of translational energy transfer when compared to the work measured here. To avoid this problem as much as possible we assume that only the velocity parallel to the surface is conserved. We can then derive an $\mathrm{N}$ value for the effective number of surface atoms for each of the previous studies. 
Table 4.4 effective mass of surface atom in Baule model for different molecule surface systems.

\begin{tabular}{|c|c|c|c|}
\hline Scattering system & $\left\langle E_{S, \text { norm }}\right\rangle /\left\langle E_{i, \text { norm }}\right\rangle$ & $\begin{array}{c}\text { Mass ratio, } \\
\mathbf{m}_{\mathbf{1}} / \mathbf{m}_{\mathbf{2}}\end{array}$ & $\mathbf{N}_{\text {Surface Atoms }}$ \\
\hline \hline $\mathrm{Ar} / \mathrm{Pt}^{83}$ & $0.6-0.7$ & 0.205 & $\mathrm{~N}_{\mathrm{Pt}}=1.8-2.3$ \\
\hline $\begin{array}{c}\mathrm{HCl}(\mathrm{v}=2) / \mathrm{Au} \text { [this } \\
\text { work] }\end{array}$ & 0.44 & 0.183 & $\mathrm{~N}_{\mathrm{Au}}=1$ \\
\hline $\mathrm{Ar} / \mathrm{Ag}^{84}$ & $0.5-0.6$ & 0.370 & $\mathrm{~N}_{\mathrm{Ag}}=2.4-3.4$ \\
\hline $\mathrm{HCl}(\mathrm{v}=2) / \mathrm{MgO}^{71}$ & 0.40 & $2.25,1.5,0.9$ & $\mathrm{~N}_{\mathrm{O}}=10 ; \mathrm{N}_{\mathrm{Mg}}=7 ; \mathrm{N}_{\mathrm{MgO}}=4$ \\
\hline $\mathrm{H}_{2} / \mathrm{Cu}^{78}$ & 0.95 & 0.031 & $\mathrm{~N}_{\mathrm{Cu}}=1-2$ \\
\hline $\mathrm{D}_{2} / \mathrm{Cu}^{78}$ & 0.875 & 0.063 & $\mathrm{~N}_{\mathrm{Cu}}=1-2$ \\
\hline $\mathrm{Ar} / \mathrm{C}^{85}$ & 0.4 & 3.33 & $\mathrm{~N}_{\mathrm{C}}=5.8-8.3$ \\
\hline $\mathrm{Xe} / \mathrm{C}^{86}$ & 0.14 & 10.9 & \begin{tabular}{c}
$\mathrm{N}_{\mathrm{C}}=25$ \\
\hline $\mathrm{N}_{2} / \mathrm{Ru}^{87}$
\end{tabular} \\
\hline $\mathrm{NO} / \mathrm{Ag}^{64}$ & $0.5-0.8$ & 0.277 & $\begin{array}{c}\mathrm{N}_{\mathrm{Ru}}=1.5-7 \text { (energy } \\
\text { dependent })\end{array}$ \\
\hline & $0.4-0.8$ & 0.278 & $\begin{array}{c}\mathrm{N}_{\mathrm{Ag}}=1-4 \text { (energy } \\
\text { dependent })\end{array}$ \\
\hline
\end{tabular}

Typical values for the effective mass of the surface lie between two and four surface atoms. Only $\mathrm{H}_{2} / \mathrm{D}_{2}$ scattering from $\mathrm{Cu}$, shows comparable translational energy transfer to the surface as I measured. ${ }^{77,78}$ It is also worth noting that the $\mathrm{H}_{2} / \mathrm{D}_{2}$ scattering is one of the few state-to-state scattering studies comparable to this work. However due to the large mass mismatch between $\mathrm{H}_{2} / \mathrm{D}_{2}$ and $\mathrm{Cu}$ the Baule limit for this system corresponds to 0.88 and 0.78 translational energy being retained in $\mathrm{H}_{2}$ and $\mathrm{D}_{2}$, respectively. This small translational energy exchange is of course experimentally more difficult to determine.

The comparison to $\mathrm{Ar} / \mathrm{Pt}^{83}$ scattering is interesting to make as the masses of $\mathrm{Ar}$ and $\mathrm{Pt}$ are comparable to that of $\mathrm{HCl}$ and $\mathrm{Au}$, and because the quality of the data is comparable to that measured here. Additionally the authors used an isoflux contour method to decompose the energy transfer to its normal component. This makes the comparison straightforward and meaningful as no angular bias exists like that described with $\mathrm{HCl}$ scattering from $\mathrm{Au}(111) .{ }^{80}$ For the $\mathrm{Ar} / \mathrm{Pt}$ system the conservation of parallel momentum was approximately verified at angles close to specular. Using the high incidence translational energy data at an incidence angle of $\theta_{\mathrm{i}}=5^{\circ}$ and extracting only the normal component the effective number of $\mathrm{Pt}$ atoms is found to be 1.8-2.3. The energy transfer for this system is substantially less than that of $\mathrm{HCl} / \mathrm{Au}$ measured here, where the effective number of $\mathrm{Au}$ atoms is 1. Similar results for Ar scattering from $\mathrm{Ag}$ were measured where the effective number of atoms was 2.4-3.6. 
NO scattering from Ag also shows, at least at high incidence energies, comparable translational inelasticity to the $\mathrm{HCl} / \mathrm{Au}$ results shown here. ${ }^{64} \mathrm{At}_{\mathrm{i}}=0.98 \mathrm{eV}$ inelasticity was well matched with an effective number of a single $\mathrm{Ag}$ atom. However as incidence energy of translation decreased the effective number of $\mathrm{Ag}$ atoms grew, for example at $\mathrm{E}_{\mathrm{i}}=0.28 \mathrm{eV}$ an effective number of $4 \mathrm{Ag}$ atoms is found. This is qualitatively similar $\mathrm{N}_{2}$ scattering from $\mathrm{Ru}(0001) .{ }^{87}$

Another meaningful comparison is the vibrationally elastic scattering of $\mathrm{HCl}(\mathrm{v}=2)$ from $\mathrm{MgO} .^{71}$ These studies were carried out at near normal incidence, and the molecules were probed in a state-to-state manner. Utilizing the Baule model here leads to some ambiguity as it is not clear what to use as the mass of the surface atom. As a comparison the effective number of atoms was used with an atom with the mass of $\mathrm{O}$ (16), $\mathrm{Mg}(24)$, and $\mathrm{MgO}$ "atoms" (40). In all cases the effective number of "atoms" was between $4(\mathrm{MgO})$, and $10(\mathrm{O})$. These experiments showed much less translational inelasticity compared to the work measured here. One can also note that in the Table 4.4 insulators tend to have higher $\mathrm{N}$ values and hence molecules scattering from them have less translational inelasticity than metals.

In contrast to the examples mentioned above, and other results shown in Table 4.4 the $\mathrm{HCl}$ scattering from $\mathrm{Au}(111)$ shows a remarkable amount of translational inelasticity. Here, at all incidence translational energies measured, the average translational energy transfer is at or beyond the Baule limit, as seen in Fig. 4.18.

The simplest interpretation of this result is that the Baule model accurately describes the results of this work. The validity of this simple impulse model to this system is not obviously inappropriate. This limit is appropriate when the incidence energies are large enough so that the $\mathrm{Au}$ atom motion occurs on a longer time scale than the collision. These time scales can be roughly estimated by combining knowledge of the incidence velocities, the length scale of the collision and the phonon spectrum of $\mathrm{Au}(111)$. The lowest velocities used in this work are 1000 $\mathrm{m} / \mathrm{s}$. We estimate the length scale of the collision from canonical van der Waals and covalent bonding of radii of $\mathrm{Au}$ and $\mathrm{Cl}$. In this way:

$$
\delta=\left(\mathrm{R}_{\mathrm{vdW}, \mathrm{Au}}+\mathrm{R}_{\mathrm{vdWCl}}\right)-\left(\mathrm{R}_{\mathrm{cov}, \mathrm{Au}}+\mathrm{R}_{\mathrm{cov}, \mathrm{Cl}}\right)=0.87 \AA
$$

This leads to a collision time scale of $\tau=2 \delta / v=1.5 \times 10^{-13} \mathrm{~s}$. This may be compared with the inverse Debye Frequency of the $\mathrm{Au}(111)$ solid, $1 / \omega_{\mathrm{D}}=2.9 \times 10^{-13} \mathrm{~s}$, reflecting the highest frequency vibration of the surface. The estimated difference in time scales, $2 / \tau \omega_{\mathrm{D}}$, is only about a factor of two for experiments where the two time scales are closest. At the highest incidence energies, it increases to about 9. This analysis suggests that an impulse approximation might be reasonably accurate, at least for the range of incidence energies of translation studied here.

My experiments probe mostly specular scattering at near normal incidence energy, a backscattering geometry which may tend to emphasize collisions with a small impact parameter. If corrugation of the interaction potential is small then backscattering can occur from any surface site. There is no evidence to suggest that the corrugation of the $\mathrm{HCl} \mathrm{Au(111)} \mathrm{potential} \mathrm{surface} \mathrm{is}$ 
large. If this is the case then the probability to scatter from surface sites where the $\mathrm{HCl}$ projectile could interact with multiple surface atoms becomes likely as opposed to scattering from solely from A-top sites. This might be, in fact, the reason for the origin of the increased effective number seen in rare gas atom scattering and scattering from insulator surfaces. Beyond this simple picture the nature of the potential energy surface and lattice properties $\mathrm{HCl} / \mathrm{Au}(111)$ may be peculiar leading to a large mechanical translational energy transfer. In this regard accurate potential surface calculations combined with theoretical dynamics studies are clearly needed.

Another possible explanation, for this large translational inelasticity, is that a significant fraction of the translational energy of the $\mathrm{HCl}$ is transferred to electronic degrees of freedom in the metal. Figure 4.19 shows the $\mathrm{HCl}(\mathrm{v}=2 \rightarrow 2)$ channel translational energy distributions. The vertical lines represent the attractive Baule limit.

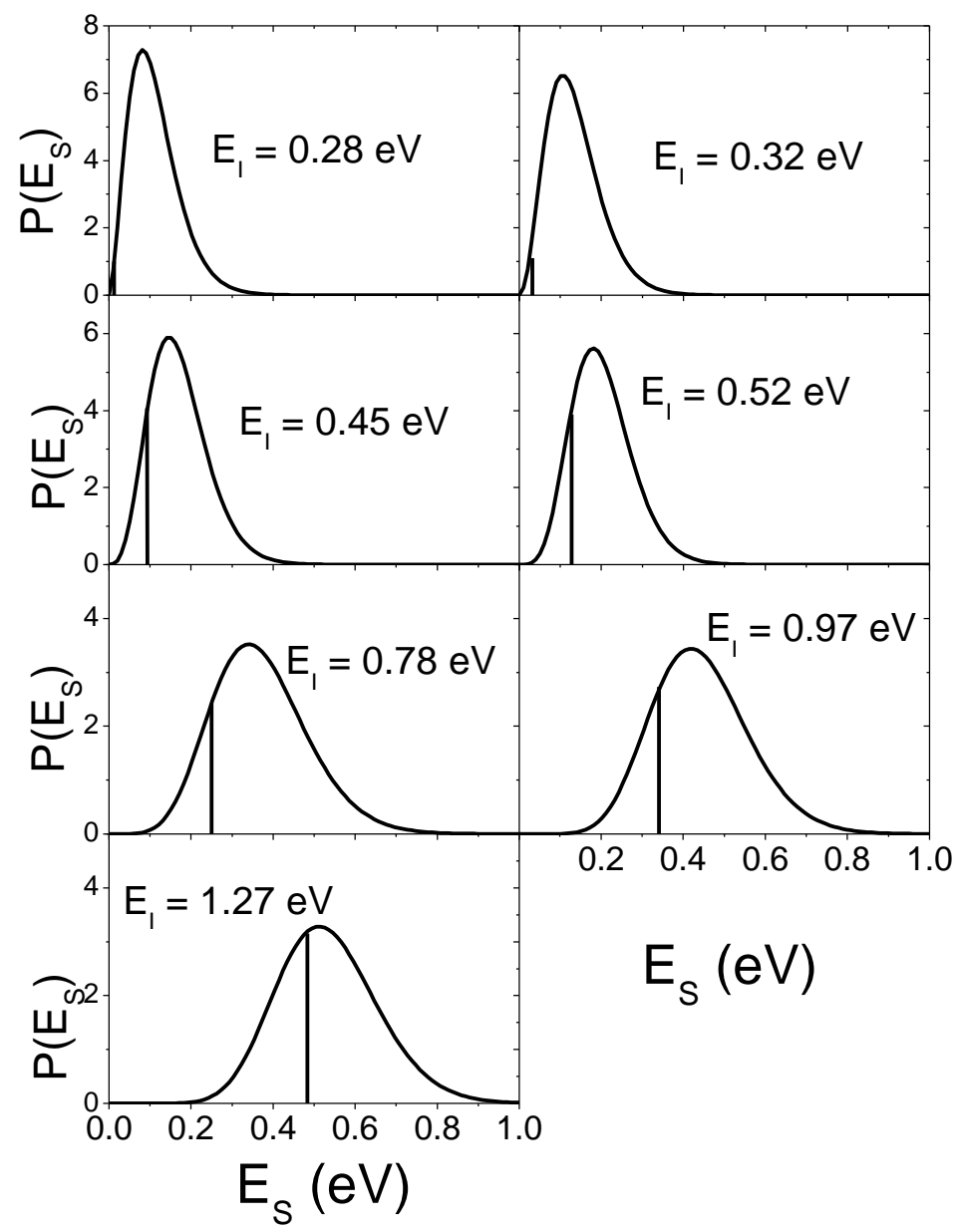

Figure 4.19 $\mathrm{P}(\mathrm{E})$ 's at six incidence translational energies of the scattered beam in the $\mathrm{HCl}(\mathrm{v}=2 \rightarrow 2)$ channel. The vertical lines represent the attractive Baule limit. 
The results shown in Fig. 4.19 allow for an estimation of the fraction of the molecules that scatter back with sub-Baule translational energies. The fraction of sub-Baule molecules depends on the incidence energy of translation, ranging from $<0.01$ at low incidence energies to $>0.20$ at higher incidence energies. It should be noted that at $\mathrm{E}_{\mathrm{i}}=1.27 \mathrm{eV}$ the signal-to-noise is much worse than at other incidence energies making the sub-Baule fraction measured there somewhat questionable. However, the fact that the $\mathrm{HCl}(\mathrm{v}=0 \rightarrow 0)$ signal in that case, which does not suffer from signal-to-noise problems is nearly identical, gives some reassurance as to the data at $\mathrm{E}_{\mathrm{i}}=1.27 \mathrm{eV}$.

Table 4.5 Fraction of scattered molecules which have a translational energy below that expected by the attractive Baule limit for the different incidence energies used in this work.

\begin{tabular}{|l|l|l|l|l|l|l|l|}
\hline $\mathbf{E}_{\mathbf{i}}, \mathbf{e V}$ & 0.28 & 0.32 & 0.45 & 0.52 & 0.78 & 0.97 & 1.27 \\
\hline $\begin{array}{l}\text { Fraction sub- } \\
\text { Baule }\end{array}$ & 0.006 & 0.04 & 0.17 & 0.19 & 0.18 & 0.22 & 0.37 \\
\hline
\end{tabular}

This large fraction of molecules, at high incidence translational energies, with translational energies below that of the Baule limit is perhaps difficult to explain with a purely mechanical model. One possible explanation for this would be coupling of the translation of the molecule to electron hole pairs in the surface. This idea is not without precedence as coupling between translational energy and electron hole pairs in metals and semi-conductors was previously seen to occur at $\mathrm{E}_{\mathrm{i}}=3-10 \mathrm{eV} .{ }^{88}$ Perhaps a bit remarkably, the present work suggests that this might also take place at energies important to thermal collisions at modest temperatures at incidence translational energies that are comparable to the physisorption well depths. If this is indeed the case then any study of $\mathrm{HCl} / \mathrm{Au}(111)$ sticking probabilities will need to include coupling to electron hole pairs of the surface in order to be accurate.

\subsection{Conclusions to $\mathrm{HCl}$ translational studies:}

I have shown that the idea of translational spectatorship is not fully true, at least for $\mathrm{HCl} / \mathrm{Au}(111)$ vibrational relaxation. I showed that a constant fraction, $26 \%$, of the vibrational energy is converted to outgoing molecular translation and that this fraction is independent on incidence translational energy. I showed that the vibrational energy lost to the surface likely couples to electron hole pair formation in the metal surface, although comparisons to first principles theory are lacking and needed. I have discussed possible mechanisms for the coupling between the vibrational and translational degrees of freedom in the molecule, leading to the breakdown of the "spectator view" of translation.

The translational energy transfer to the surface in the vibrationally elastic case was carried out for two incidence vibrational states $\mathrm{HCl}(\mathrm{v}=2)$ and $\mathrm{HCl}(\mathrm{v}=0)$. Here I showed that there is no dependence in the translational energy transfer on the incident vibrational state. In all cases the translational energy transferred to the surface is quite large. I made a comparison to a simple 
impulsive binary model and found that a substantial fraction of the molecules at high incidence energy of translation had translational energies below that expected in a purely mechanical mechanism.

\subsection{NO translational energy transfer}

\subsection{1a Vibrational elastic scattering:}

In an effort to elucidate the mechanism of translation energy transfer in gas surface scattering, I carried out a comparable study to that of $\mathrm{HCl}(\mathrm{v}=2 \mathrm{~J}=1)$ scattering from a $\mathrm{Au}(111)$. Here NO $(\mathrm{v}=3 \mathrm{~J}=1.5)$ was prepared via optical overtone pumping and was scattered from a $\mathrm{Au}(111)$ surface. The recorded times of flight were converted to energy distributions in an analogous fashion to the experiments of $\mathrm{HCl}$ on $\mathrm{Au}(111)$. One limiting factor to the resolution of $\mathrm{NO}(\mathrm{v}=3$ $\mathrm{J}=1.5)$ scattering from $\mathrm{Au}(111)$ is that the probe laser could not be focused due to nonresonant ionization. This causes the REMPI laser to act as a line detector instead of a point detector, the case when the probe laser is focused. This leads to uncertainty in the flight distance. However changes to the average energy due to this uncertainty can be calculated. Assuming the molecules are scattered cylindrically symmetrically the FWHM of the angular distribution is $11.6 \mathrm{~mm}$. This means molecules scattered at the FWHM of the angular distribution travel $1.2 \mathrm{~mm}$ farther than the $13.5 \mathrm{~mm}$ of molecules that are specularly scattered. This leads to a change in the average final translational energy of around 20\%. However since most molecules scatter with a final flight distance less than that of the FWHM this effect is even less. However, in general, the line detector biases the results towards lower translational energies. 


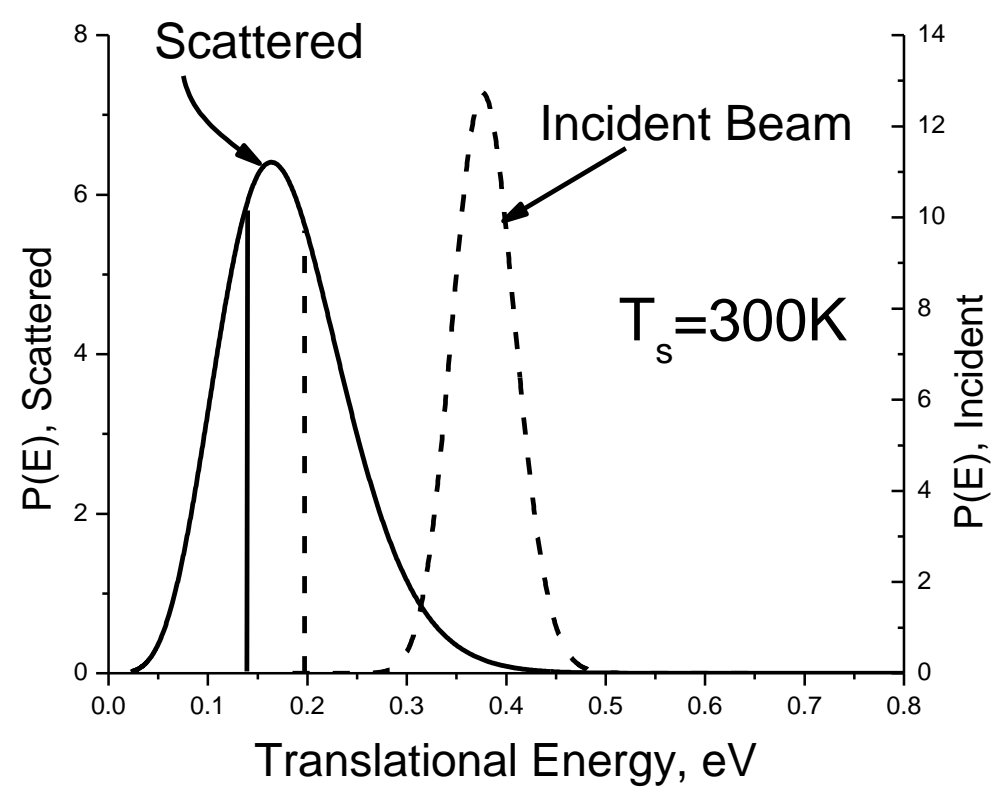

Figure 4.20 Translational energy distributions for incident, dashed line, and scatted $\mathrm{NO}(\mathrm{v}=3, \mathrm{~J}=2.5 \rightarrow 3 \mathrm{~J}=7.5)$, solid line, for $\mathrm{E}_{\mathrm{i}}=0.378 \mathrm{eV}$ and $\mathrm{T}_{\mathrm{s}}=300 \mathrm{~K}$. The black vertical line represents the attractive Baule limit, the dashed vertical line represents the Baule limit.

The average energy for vibrationally elastic scattering $\mathrm{NO}(\mathrm{v}=3, \mathrm{~J}=2.5 \rightarrow 3 \mathrm{~J}=7.5)$ was $0.182 \mathrm{eV}$ when scattered from a room temperature surface with an $\mathrm{E}_{\mathrm{i}}=0.378 \mathrm{eV}$. This means that on average 0.52 of the translational energy was transferred to the surface. This is comparable to the translational energy transfer of $\mathrm{HCl}$ from $\mathrm{Au}(111)$ shown in the previous section, however it is important to note that $\mathrm{NO}$ is lighter than $\mathrm{HCl}, 30$ and 36 amu respectively. This means that according to the Baule model there should be a smaller fraction of the translational energy transferred to the surface by $\mathrm{NO}$ compared to $\mathrm{HCl}$. According to the attractive Baule limit, for NO scattering from Au 0.46 of the translational energy should be transferred to the surface. This would mean that 0.30 of the molecules are scattered with translational energy below the attractive Baule limit. See Fig. 4.20.

Studies of translational inelasticity for vibrationally elastic NO scattering from $\operatorname{Ag}(111)$ were previously reported. ${ }^{64}$ In that work, the fraction of translational energy transferred to the surface depended on the incidence kinetic energy. The largest fraction of kinetic energy was transferred at high incidence energies. For example at $\mathrm{E}_{\mathrm{i}}=0.9 \mathrm{eV}$, the scattered molecule transferred around 0.60 of its perpendicular translational energy to the surface. The conditions that would perhaps best apply as a comparison to the work here is the results of $\mathrm{E}_{\mathrm{i}}=0.475 \mathrm{eV}$ with an incident angle of $30^{\circ}$ relative to surface normal. This gives a normal $\mathrm{E}_{\mathrm{i}}=0.411 \mathrm{eV}$, which is only slightly higher than that used here. The scattered normal translational energy was measured to be $0.26 \mathrm{eV}$, giving a translational energy transfer of 0.40 to the surface. 
NO scattering from $\mathrm{Au}(111)$ showed more translational energy transfer to the surface than NO scattering from $\mathrm{Ag}(111)$, despite the fact that $\mathrm{Ag}$ atoms are lighter than $\mathrm{Au}$ and according to the Baule model, should be able to absorb more translational energy of the incoming NO than would $\mathrm{Au}$. In fact, the Baule limit for an NO/Ag interaction is that 0.70 of the translational energy would transfer to the surface. This means the average scattered translational energy transfer to the surface of 0.40 is much less than the expected 0.70 from the Baule limit. Although no translational energy distributions are shown in the NO/Ag work, it is likely that most of the molecules have translational energies above the Baule limit. Referencing Table 4.1 it is clear that NO scattering from $\mathrm{Au}(111)$ shows the strongest divergence from the Baule limit yet measured.

This leads to the question of what causes this deviation from the Baule limit. This was discussed in depth in the section on $\mathrm{HCl}$ scattering. I will revisit the ideas here in the context of how NO scattering from $\mathrm{Au}(111)$ elucidates the mechanism.

One perhaps interesting route to exploring the coupling between translational energy of an incoming molecule and electronic degrees of freedom in the metal would be to calculate, or better yet measure, the vibrational lifetime of the translational mode of the adsorbed molecule and compare this to the divergence from the Baule model. In fact calculations of the coupling between the molecules translational mode and the surface electrons could be carried on in a similar fashion to the calculations for vibrational coupling to surface electrons. ${ }^{41,43}$

\subsection{1b Vibrational inelastic scattering:}

As with $\mathrm{HCl}$ scattering from $\mathrm{Au}(111)$, the final translational energy of $\mathrm{NO}$ was measured as a function of its final vibrational state. Previous reports of NO undergoing vibrational relaxation on a $\mathrm{Au}(111)$ surface have suggested that translation is a spectator with regards to vibrational energy change. ${ }^{10}$ It should be noted that this was a peripheral aspect of the studies, and the resolution of the experiment did not match the resolution of the experiments here. In this work, I measured vibrationally elastic $\mathrm{NO}(\mathrm{v}=3 \rightarrow 3)$ and inelastic $\mathrm{NO}(\mathrm{v}=3 \rightarrow 2)$ TOF and converted them to translational energy distributions. Unfortunately $\mathrm{NO}(\mathrm{v}=3 \rightarrow 1)$ and $\mathrm{NO}(\mathrm{v}=3 \rightarrow 0)$ were unable to be measured due to the native population of these states in the incident beam. A comparison of the vibrationally elastic and inelastic channels is shown in Fig. 4.21. 


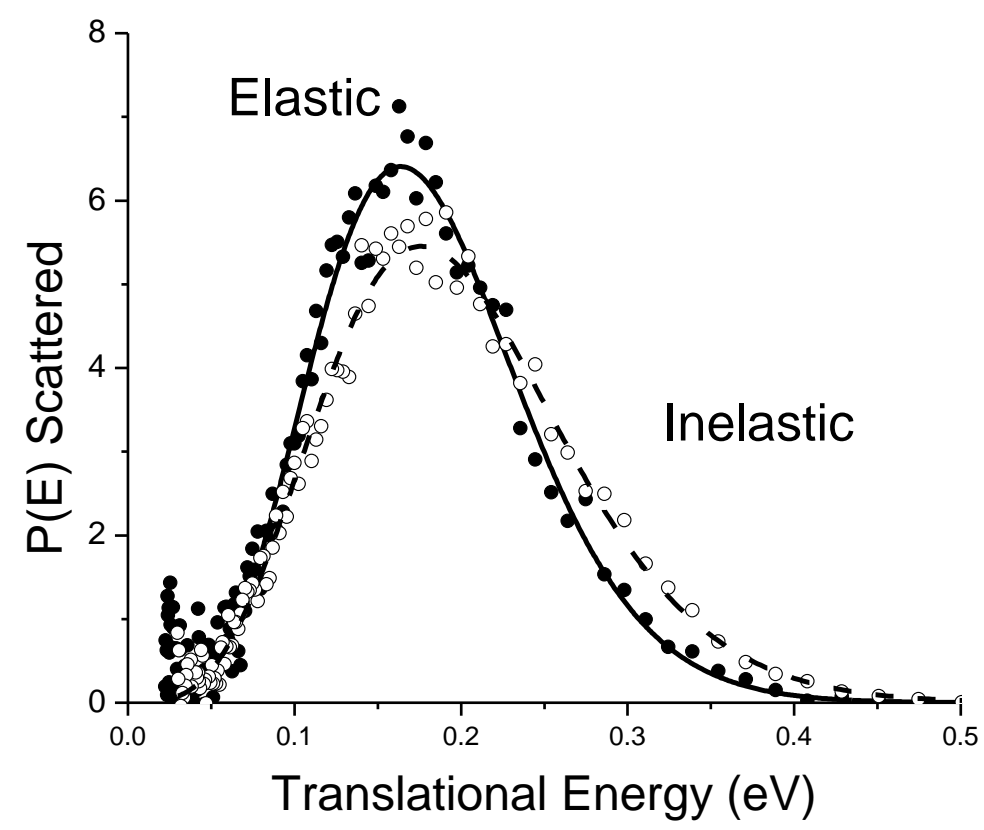

Figure 4.21 Translational energy distributions for scattered $\mathrm{NO}$ in the vibrationally elastic case $\mathrm{NO}(\mathrm{v}=3 \rightarrow 3)$, filled circles, and inelastic case $\mathrm{NO}(\mathrm{v}=3 \rightarrow 2)$, empty circles with an $\mathrm{E}_{\mathrm{i}}=0.378 \mathrm{eV}$ and $\mathrm{T}_{\mathrm{s}}=300 \mathrm{~K}$. The solid and dashed lines are fits, explained in section 4 , to the vibrationally elastic and inelastic data, respectively.

The vibrationally inelastic channel shows only a slight broadening and increase in translational energy as compared to the vibrationally elastic channel. Indeed, the differences between the two channels are likely less important than the similarities. The average translational energy difference between vibrationally elastic and inelastic channels is found to be only $0.017 \mathrm{eV}$ corresponding to $7.2 \%$ of the vibrational energy loss. This shows that the spectator view is almost true for $\mathrm{NO}$ scattering from $\mathrm{Au}(111)$ and this is substantially less than the $0.082 \mathrm{eV}$, or $26 \%$, energy difference seen in $\mathrm{HCl}(\mathrm{v}=2 \rightarrow 1)$ vibrational relaxation.

Although this effect is smaller in $\mathrm{NO}$ than that $\mathrm{HCl}$, it is still noticeable and may have dramatic effects on the scattering process, especially when multi-quantum vibrational transitions occur. For example when $\mathrm{NO}(\mathrm{v}=15)$ is scattered from $\mathrm{Au}(111)$ the predominant process is multiquantum vibrational relaxation of $\sim 8$ quanta. If $7.2 \%$ of this energy were to couple to translation the scattered molecules would increase their translational energy by $\sim .1 \mathrm{eV}$. This amount of coupling could clearly influence interactions such as sticking.

The mechanisms of the coupling between vibration and translation of the molecule during interaction with the surface described in the section of $\mathrm{HCl}$ scattering from $\mathrm{Au}(111)$ will again be discussed here. One possibility that was dismissed in the case of $\mathrm{HCl}$ scattering from $\mathrm{Au}(111)$ was that some of the molecules underwent relaxation via a mechanical mechanism and some via coupling to surface electrons. As seen in Fig. 4.21 there is no evidence of a bimodal distribution and this idea is again disregarded. 
Another possibility is that only specific orientations of the NO molecule or specific sites on the $\mathrm{Au}(111)$ crystal allow for relaxation of the $\mathrm{NO}$ and these sites are also locations where less translational energy is transferred to the surface. This possibility cannot be excluded, especially as calculations have indicated that coupling to electrons in the metal is strongly dependent on the orientation of the $\mathrm{NO}$ molecule. ${ }^{42}$

What was deemed from the interpretation of the $\mathrm{HCl}$ scattering from $\mathrm{Au}(111)$ as the most likely mechanism for translation-vibration coupling, is that for vibrational relaxation an electron jumps to the molecule forming an ion. This ion induces a positive image charge on the surface, leading to an attraction of the molecule to the surface. The scattering event takes place, albeit at a higher incidence translational energy and upon vibrational relaxation the molecule gives the electron back to the surface and flies away, albeit with slightly more translational energy. This comparison of $\mathrm{HCl}$ to $\mathrm{NO}$ scattering is quite interesting. This would indicate that the lifetime of the $\mathrm{HCl}$ ion is substantially longer than the NO ion in the scattering process. Although these thoughts are interesting, they remain highly speculative.

Perhaps the most likely explanation for these results is direct coupling between NO molecular vibration and translational energy during the scattering event. Here, a complex interplay involving the electrons of the surface as well as two degrees of freedom in the molecule would exchange energy. How this interaction takes place is not clear.

In this section NO translational energy distributions were measured for molecules scattered from a $\mathrm{Au}(111)$ surface. The translational energy distribution was measured for both vibrationally elastic scattering $\mathrm{NO}(\mathrm{v}=3 \rightarrow 3)$ and inelastic $\mathrm{NO}(\mathrm{v}=3 \rightarrow 2)$ channels. The translational energy transfer to the surface in the vibrationally elastic case showed that more than half of the molecules had translational energies below the Baule limit. In the vibrationally inelastic case the translational energy distribution was slightly broader than the elastic case and more energetic. Mechanisms for energy transfer to the surface were discussed in detail in both cases. 


\section{Derivation of absolute vibrational excitation probabilities:}

\subsection{Overview of experimental principles:}

In this section, I show how to derive absolute vibrational excitation probabilities, $\mathrm{P}_{0-\mathrm{v}}$, from laboratory observations of REMPI signal strengths, for $\mathrm{NO}(0 \rightarrow \mathrm{v})$ transitions resulting from collisions with the (111) surface of solid gold. We begin by defining $\mathrm{P}_{0-\mathrm{v}}$ as the ratio of scattered molecules in state $\mathrm{v}$ to all scattered molecules, for the case where almost all NO molecules are initially in $\mathrm{NO}(\mathrm{v}=0)$

$$
P_{0-\mathrm{v}} \equiv \frac{N_{\mathrm{v}}}{\sum_{i} N_{i}}
$$

When the sum of the vibrational excitation probabilities is much less than one, as in this work, Eq. (5.1) can be simplified as follows.

$$
P_{0-\mathrm{v}} \cong \frac{N_{\mathrm{v}}}{N_{0}} \equiv \frac{\text { Number of scattered molecules in state } \mathrm{v}}{\text { Number of scattered molecules in } \mathrm{v}=0}
$$

Thus, by finding the ratio of $N_{\mathrm{v}}$ to $N_{0}$, we obtain absolute vibrational excitation probabilities in a conceptually simple way.

The practical requirements of implementing this simple strategy are, however, rather complicated. Specifically, we require vibrational state specific information, such as scattering angular, $\Theta_{\mathrm{v}}(\theta)$, and rotational population, $\mathrm{n}_{\mathrm{v}}(\vec{\jmath})$, distributions. Here $\theta$ represents the scattering angle with respect to the surface normal. $\vec{j}$ represents the quantum numbers of the scattered molecules probed by the $(1+1)$ REMPI detection scheme. In general, one also needs rotational line strengths, $S\left(\vec{J}_{\Sigma}, \vec{\jmath}\right)$, and Franck-Condon factors, $Q\left(\mathrm{v}_{\Sigma}, \mathrm{v}\right)$, for the resonant $1^{\text {st }}$ photon absorption to the $\mathrm{A}^{2} \Sigma^{+}$state of NO. Here, $\vec{J}_{\Sigma}$ and $\mathrm{v}_{\Sigma}$ represent the rotational and vibrational quantum number of the intermediate, electronically-excited state used in the $(1+1)$ REMPI scheme. We must also consider the wavelength dependent ionization cross-section of the intermediate, electronically-excited state, $\sigma_{\mathrm{v}_{\Sigma}}\left(\lambda_{\text {laser }, \mathrm{v}}\right)$, where $\lambda_{\text {laser, } \mathrm{v}}$ is the laser wavelength used to detect state $\mathrm{v}$ via state $\mathrm{v}_{\Sigma}$ by REMPI. The REMPI signal also depends on the time delay, $t$, between the opening of the pulsed valve and the firing of the pulsed detection laser. This is described by a time dependent function, $\Delta_{\mathrm{v}}(t)$. This function may be different for each vibrational channel as the velocity, $u_{\mathrm{v}}$, of the scattered molecules depends on the final vibrational state. The time delay, $\tau_{\mathrm{v}}$, may be chosen differently by the experimentalist for each $\mathrm{v}$-state probed, for reasons of convenience.

Noting that REMPI signals are proportional to molecular density, are a function of the laser power, $f\left(\mathrm{I}_{\mathrm{v}}\right)$, and the MCP gain, $\Gamma_{\mathrm{v}}$, we arrive at a relationship between REMPI signal strength, $S I G_{v}\left(\vec{J}_{\Sigma}, \vec{\jmath}\right)$, and $\mathrm{n}_{\mathrm{v}}(\vec{\jmath})$, shown below. 


$$
\begin{aligned}
& S I G_{\mathrm{v}}\left(\vec{J}_{\Sigma}, \vec{J}, \theta_{\mathrm{v}}, \tau_{\mathrm{v}}\right) \\
& =\frac{n_{\mathrm{v}}(\vec{\jmath})}{u_{\mathrm{v}}} \times S\left(\vec{J}_{\Sigma}, \vec{\jmath}\right) \times \Theta_{\mathrm{v}}\left(\theta_{\mathrm{v}}\right) \times Q\left(\mathrm{v}_{\Sigma}, \mathrm{v}\right) \times \sigma_{\mathrm{v} \Sigma}\left(\lambda_{\text {laser }, \mathrm{v}}\right) \times \Delta_{\mathrm{v}}\left(\tau_{\mathrm{v}}\right) \times \Gamma_{\mathrm{v}} \times f\left(I_{\mathrm{v}}\right)
\end{aligned}
$$

Here, $u_{\mathrm{v}}$ is needed for the density-to-flux conversion. $\theta_{\mathrm{v}}$ and $\tau_{\mathrm{v}}$ are chosen for convenience by the experimentalist.

We can invert Eq. (5.3) as follows.

$$
n_{\mathrm{v}}(\vec{\jmath})=\frac{S I G_{\mathrm{v}}\left(\vec{\jmath}_{\Sigma}, \vec{\jmath}, \theta_{\mathrm{v}}, \tau_{\mathrm{v}}\right) \times u_{\mathrm{v}}}{S\left(\vec{J}_{\Sigma}, \vec{\jmath}\right) \times \Theta_{\mathrm{v}}\left(\theta_{\mathrm{v}}\right) \times Q\left(\mathrm{v}_{\Sigma}, \mathrm{v}\right) \times \sigma_{\mathrm{v} \Sigma}\left(\lambda_{\text {laser }, \mathrm{v}}\right) \times \Delta_{\mathrm{v}}\left(\tau_{\mathrm{v}}\right) \times \Gamma_{\mathrm{v}} \times f\left(I_{\mathrm{v}}\right)}
$$

To obtain $N_{\mathrm{v}}$, we must sum over the populations of all rotational states, $|\vec{\jmath}\rangle$.

$$
\begin{aligned}
N_{\mathrm{v}}=\sum_{\vec{\jmath}} n_{\mathrm{v}}(\vec{\jmath}) & \\
= & \sum_{\vec{j}} \frac{S I G_{\mathrm{v}}\left(\vec{\jmath}_{\Sigma}, \vec{\jmath}, \theta_{\mathrm{v}}, \tau_{\mathrm{v}}\right) \times u_{\mathrm{v}}}{S\left(\vec{J}_{\Sigma}, \vec{\jmath}\right) \times \Theta_{\mathrm{v}}\left(\theta_{\mathrm{v}}\right) \times Q\left(\mathrm{v}_{\Sigma}, \mathrm{v}\right) \times \sigma_{\mathrm{v} \Sigma}\left(\lambda_{\text {laser }, \mathrm{v}}\right) \times \Delta_{\mathrm{v}}\left(\tau_{\mathrm{v}}\right) \times \Gamma_{\mathrm{v}} \times f\left(I_{\mathrm{v}}\right)}
\end{aligned}
$$

Plugging this result back into Eq. (5.2) yields the following.

$$
P_{0-\mathrm{v}} \cong \frac{N_{\mathrm{v}}}{N_{0}}=\frac{\sum_{\vec{j}} \frac{S I G_{\mathrm{v}}\left(\vec{J}_{\Sigma}, \vec{\jmath}, \theta_{\mathrm{v}}, \tau_{\mathrm{v}}\right) \times u_{\mathrm{v}}}{S\left(\vec{J}_{\Sigma}, \vec{\jmath}\right) \times \Theta_{\mathrm{v}}\left(\theta_{\mathrm{v}}\right) \times Q\left(\mathrm{v}_{\Sigma}, \mathrm{v}\right) \times \sigma_{\mathrm{v} \Sigma}\left(\lambda_{\text {laser }, \mathrm{v}}\right) \times \Delta_{\mathrm{v}}\left(\tau_{\mathrm{v}}\right) \times \Gamma_{\mathrm{v}} \times f\left(I_{\mathrm{v}}\right)}}{\sum_{\vec{\jmath}} \frac{S I G_{0}\left(\vec{J}_{\Sigma}, \vec{\jmath}, \theta_{0}, \tau_{0}\right) \times u_{0}}{S\left(\vec{J}_{\Sigma}, \vec{\jmath}\right) \times \Theta_{0}\left(\theta_{0}\right) \times Q\left(\mathrm{v}_{\Sigma}, 0\right) \times \sigma_{\mathrm{v} \Sigma}\left(\lambda_{\text {laser }, 0}\right) \times \Delta_{0}\left(\tau_{0}\right) \times \Gamma_{0} \times f\left(I_{0}\right)}}
$$

This rather complicated looking expression can be simplified, since under our experimental conditions the $1^{\text {st }}$ step in the $(1+1)$ REMPI scheme is saturated. This leads to the key formula needed to derive absolute vibrational excitation probabilities.

$$
P_{0-\mathrm{v}} \cong \frac{N_{\mathrm{v}}}{N_{0}}=\left(\frac{\sum_{\vec{j}} S I G_{\mathrm{v}}\left(\vec{J}_{\Sigma}, \vec{\jmath}, \theta_{\mathrm{v}}, \tau_{\mathrm{v}}\right)}{\sum_{\vec{\jmath}} S I G_{0}\left(\vec{J}_{\Sigma}, \vec{\jmath}, \theta_{0}, \tau_{0}\right)}\right) \frac{u_{\mathrm{v}} x \Theta_{0}\left(\theta_{0}\right) \times \sigma_{\mathrm{v} \Sigma}\left(\lambda_{\text {laser }, 0}\right) \times \Delta_{0}\left(\tau_{0}\right) \times \Gamma_{0} \times f\left(I_{0}\right)}{u_{0} \times \Theta_{\mathrm{v}}\left(\theta_{\mathrm{v}}\right) \times \sigma_{\mathrm{v} \Sigma}\left(\lambda_{\text {laser }, \mathrm{v}}\right) \times \Delta_{\mathrm{v}}\left(\tau_{\mathrm{v}}\right) x \Gamma_{\mathrm{v}} \times f\left(I_{\mathrm{v}}\right)}
$$

In the following sections, I describe how this approach is carried out in practice. Briefly, I carry out measurement of $\sum_{\vec{\jmath}} \operatorname{SI} G_{\mathrm{v}}\left(\vec{J}_{\Sigma}, \vec{\jmath}, \theta_{\mathrm{v}}, \tau_{\mathrm{v}}\right)$ by conducting laser scans over an entire vibronic band at chosen values of $\theta_{\mathrm{v}}, \tau_{\mathrm{v}}, \Gamma_{\mathrm{v}}$ and $I_{\mathrm{v}}$. I choose vibronic bands to avoid overlapping transitions from other scattered vibrational states. This consideration determines $\lambda_{\text {laser, } v}$. I adjust $\Gamma_{\mathrm{v}}$ to avoid MCP saturation and $I_{\mathrm{v}}$ to ensure saturation of the resonant step in the REMPI scheme. In separate but similar experiments I measure $\sum_{\vec{\jmath}} \operatorname{SI}_{0}\left(\vec{J}_{\Sigma}, \vec{J}, \theta_{0}, \tau_{0}\right)$, choosing $\theta_{0}, \tau_{0}, \Gamma_{0}$ and $I_{0}$ based on similar considerations. In the absence of trapping $N_{0}$ should be independent of surface temperature, and this was verified experimentally. Thus for each incidence energy of translation a single value of $N_{0}$ typically measured at a surface temperature 
of $300 \mathrm{~K}$, is used to calculate $\mathrm{P}_{0-\mathrm{v}}$ at elevated surface temperatures. I also carry out separate angular distributions measurements, $\Theta_{\mathrm{v}}$ and $\Theta_{0}$, which for analysis are modeled as normalized Gaussian functions. Time delay distributions, $\Delta_{\mathrm{v}}$ and $\Delta_{0}$, are measured and analyzed numerically. I used literature values of ionization cross-sections $\sigma_{\mathrm{v} \Sigma}\left(\lambda_{\text {laser }}\right)^{89,90}$. The laser power dependence is measured and fit to a suitable (but empirical) function. I also experimentally characterize the MCP gain. With such data in hand, I generate a single value of $P_{0-\mathrm{v}}$ at a specific choice of $\mathrm{v}$, incidence energy of translation, $\mathrm{E}_{\mathrm{i}}$, and surface temperature, $\mathrm{T}_{\mathrm{S}}$. This procedure was repeated more than 140 times for different values of $\mathrm{E}_{\mathrm{i}}$ and $\mathrm{T}_{\mathrm{S}}$ for $\mathrm{NO}(\mathrm{v}=0 \rightarrow 1)$, and $\mathrm{NO}(\mathrm{v}=0 \rightarrow 2)$. I strove to create a sufficiently comprehensive data set that allows meaningful comparisons to the next generation of nonadiabatic energy transfer theories. Six different incidence energies of translation were used in this work. I carried out quantification of the incident beam as in section 4. The parameters describing the speed distributions of the incident beam are shown in Table 5.1. $\left\langle\mathrm{E}_{\mathrm{i}}\right\rangle$ is the average translational energy, $\mathrm{v}_{0}$ the average velocity. For more detailed explanation of these parameters please see section 4 or Appendix 1.

Table 5.1 Parameters describing the speed distributions for the incident beam

\begin{tabular}{|c|c|c|c|c|}
\hline Mixing Ratios & $\left\langle E_{i}\right\rangle, \mathrm{eV}$ & $\sqrt{\left|\left\langle E_{i}^{2}\right\rangle-\left\langle E_{i}\right\rangle^{2}\right|}, \mathrm{eV}$ & $v_{0}, \mathrm{~m} / \mathrm{s}$ & $\alpha, \mathrm{m} / \mathrm{s}$ \\
\hline \hline $1 \% \mathrm{NO} / 99 \% \mathrm{H}_{2}$ & 1.05 & 0.110 & 2574 & 195 \\
\hline $2.5 \% \mathrm{NO} / 97.5 \% \mathrm{H}_{2}$ & 0.93 & 0.071 & 2431 & 133 \\
\hline $6.5 \% \mathrm{NO} / 93.5 \% \mathrm{H}_{2}$ & 0.63 & 0.084 & 1973 & 191 \\
\hline $10 \% \mathrm{NO} / 90 \% \mathrm{H}_{2}$ & 0.45 & 0.050 & 1676 & 136 \\
\hline $5 \% \mathrm{NO} / 25 \% \mathrm{~N}_{2} / 70 \% \mathrm{H}_{2}$ & 0.28 & 0.041 & 1305 & 140 \\
\hline $7.5 \% \mathrm{NO} / 92.5 \% \mathrm{~N}_{2}$ & 0.11 & 0.012 & 818 & 69 \\
\hline
\end{tabular}

\subsection{REMPI scans of isolated vibronic bands; $\sum_{\vec{j}} \boldsymbol{S I} G_{\mathbf{v}}\left(\overrightarrow{J_{\Sigma}}, \vec{\jmath}, \theta_{\mathbf{v}}, \boldsymbol{\tau}_{\mathbf{v}}\right)$ :}

For each incidence energy shown in Table 5.1, (1+1) REMPI spectra probing $\mathrm{NO}(\mathrm{v}=0,1$, and 2) were recorded between $T_{\mathrm{S}}=300$ and $985 \mathrm{~K}$. See examples shown in Fig. 5.1. The entire REMPI spectrum was integrated giving the spectral intensity, $\sum_{\vec{\jmath}} S I G_{\mathrm{v}}\left(\vec{J}_{\Sigma}, \vec{\jmath}, \theta_{\mathrm{v}}, \tau_{\mathrm{v}}\right)$, for each vibrational state. At each value of $E_{\mathrm{i}}$, REMPI spectra like those of Fig. 5.1 are measured at many values of $T_{\mathrm{S}}$. Note that for convenience the $\mathrm{NO}(\mathrm{v}=0)$ spectra are taken at $T_{\mathrm{S}}=300 \mathrm{~K}$. 


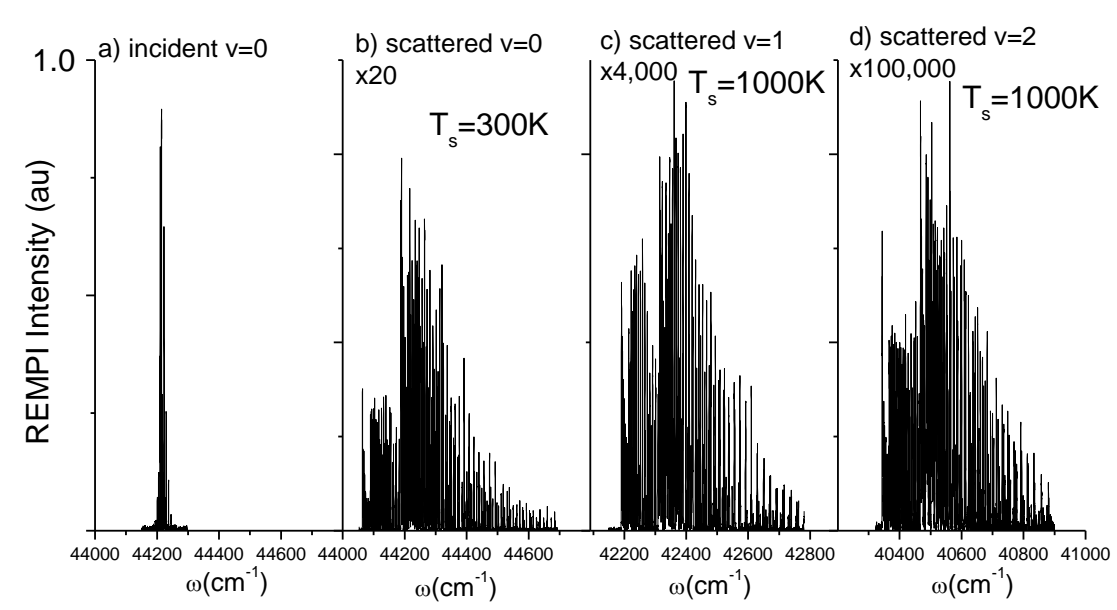

Figure 5.1 Illustrative REMPI spectra of NO before and after scattering from $\mathrm{Au}(111)$ at $\mathrm{E}_{\mathrm{i}}=0.63 \mathrm{eV}$. a) $\mathrm{NO}(\mathrm{v}=0)$ probed via the $\gamma_{00}$ band prior to collision with the surface. b) Scattered $N O(v=0)$ probed via the $\gamma_{00}$ band. c) Scattered $\mathrm{NO}(\mathrm{v}=1)$ probed via the $\gamma_{01}$ band. d) Scattered $\mathrm{NO}(\mathrm{v}=2)$ probed via the $\gamma_{02}$ band. Intensities are corrected for differences in laser power, $\mathrm{f}\left(\mathrm{I}_{\mathrm{v}}\right)$, and MCP gain, $\Gamma_{\mathrm{v}}$. The spectra $\left.\mathrm{b}\right) \mathrm{c}$ ) and d) are used to calculate the absolute excitation probabilities.

An estimate of the vibrational excitation probability can already be drawn by inspection of the intensity of the REMPI spectra shown in Fig. 5.1, which have only been corrected for differences in MCP gain and laser power. We can estimate that $\sum_{\vec{j}} S I G_{0}\left(\vec{J}_{\Sigma}, \vec{\jmath}, \theta_{0}, \tau_{0}\right)$ is $100 x$ larger than $\sum_{\vec{\jmath}} S I G_{1}\left(\vec{J}_{\Sigma}, \vec{\jmath}, \theta_{1}, \tau_{1}\right)$, which is $25 x$ larger than $\sum_{\vec{\jmath}} S I G_{2}\left(\vec{J}_{\Sigma}, \vec{\jmath}, \theta_{2}, \tau_{2}\right)$. This already gives the correct excitation probabilities within about a factor of two, neglecting all other factors. This is worth noting, as it means that all of the other factors used in the determination of the absolute excitation probabilities, which will later be described result in a net correction on the order of a factor of two.

The NO molecules are probed via $\mathrm{A}^{2} \mathrm{X}^{+}(0) \leftarrow \mathrm{X}^{2} \Pi(\mathrm{v})$. The $\gamma_{0 \mathrm{v}}$ bands were chosen due to their negligible spectral overlap with other bands of the $\gamma$-system and REMPI transitions through other intermediate electronic states. Of the bands used in this work, the $\gamma_{02}$ is a good example showing artifacts due to overlapping bands are unimportant. Consider that the nearest overlapping band is $\gamma_{13}$. Overlapping lines from this band are unlikely to be a substantial problem as $\mathrm{NO}(\mathrm{v}=3)$ is much less populated than $\mathrm{NO}(\mathrm{v}=2)$. Preliminary results show that $\mathrm{NO}(\mathrm{v}=3)$ is at least a factor of 10 less populated than $\mathrm{NO}(\mathrm{v}=2)$ under the experimental conditions used in this work. This is demonstrated in Fig. 5.2. Note that all of the observed lines can be assigned to $\gamma_{02}$ band, even those in the inset, where the band-head of $\gamma_{13}$ is expected. This shows that spectral overlap with other bands is avoided and a simple integration over the entire REMPI spectrum yields, in this case, $\sum_{\vec{\jmath}} S I G_{2}\left(\vec{\jmath}_{\Sigma}, \vec{\jmath}, \theta_{2}, \tau_{2}\right)$. 


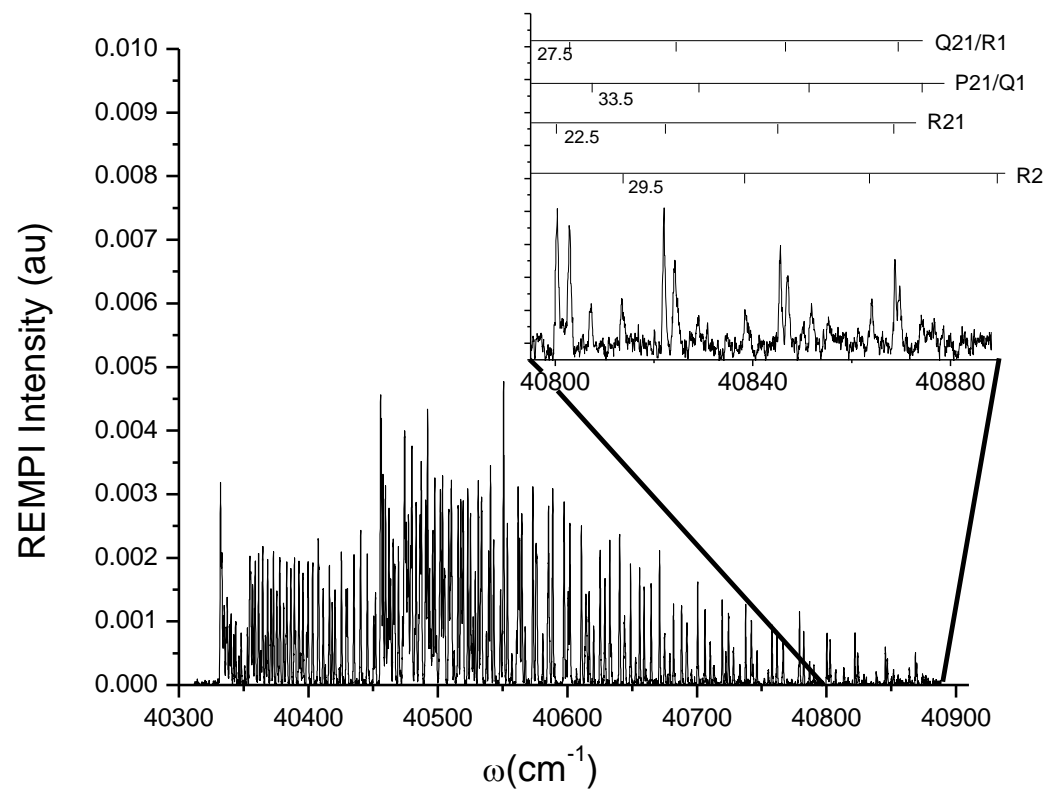

Figure 5.2 Absence of overlapping bands in the REMPI detection of scattered NO. (1+1) REMPI of scattered $\mathrm{NO}(\mathrm{v}=2)$ at $\mathrm{E}_{\mathrm{i}}=0.63 \mathrm{eV}$ and $\mathrm{T}_{\mathrm{s}}=1000 \mathrm{~K}$. The inset shows the spectral region of $\gamma_{02}$ where overlap with the band-head of $\gamma_{13}$, the nearest overlapping REMPI band, is expected. The comb in the inset indicates four branches in the $\gamma_{02}$ band. All observable peaks can be assigned to high J-states of the $\gamma_{02}$ band.

\subsection{Angular distributions; $\Theta_{\mathrm{v}}\left(\theta_{\mathrm{v}}\right)$ :}

The angular distributions of scattered molecules are, in principle, dependent upon: $T_{\mathrm{S}}, E_{\mathrm{i}}$ and $\mathrm{v}$. Broader angular distributions result in smaller REMPI signals for the same scattering flux. The $(1+1)$ REMPI used in this work employs an unfocussed laser, meaning the detection volume is cylindrical with the long axis along the laser propagation direction and more than $2 \mathrm{~cm}$ long. This means the observed REMPI signal at constant flux is linearly proportional to $\Theta_{\mathrm{v}}\left(\theta_{\mathrm{v}}\right)$.

Angular distributions of the scattered vibrationally excited molecules were measured for each incidence energy described in the experimental section at a minimum of three surface temperatures. For convenience $\Theta_{0}$ was measured at $T_{\mathrm{S}}=300 \mathrm{~K}$. REMPI spectra of scattered molecules were typically recorded off the peak of the angular distribution to avoid the probe laser spatially overlapping the incident molecular beam.

Each measured angular distribution was fitted to a normalized Gaussian function.

$$
\Theta_{\mathrm{v}}(\Theta)=\frac{e^{-\frac{1}{2}\left(\frac{\theta-\theta_{0}}{\sigma}\right)^{2}}}{\sqrt{2 \pi} \sigma}
$$

Here, $\theta_{0}$ is the scattering angle of peak intensity and $\sigma$ is related to the full width at half maximum $(F W H M=2 \sigma \sqrt{2 \ln 2}$ ). I used linear interpolation of the width parameter, $\sigma$, to characterize angular distributions at temperatures where measurements were not carried out. This 
is justified by the observation that the measured $\Theta_{\mathbf{v}}$ 's vary only slightly over the range of temperatures used in this work. See Fig. 5.3.

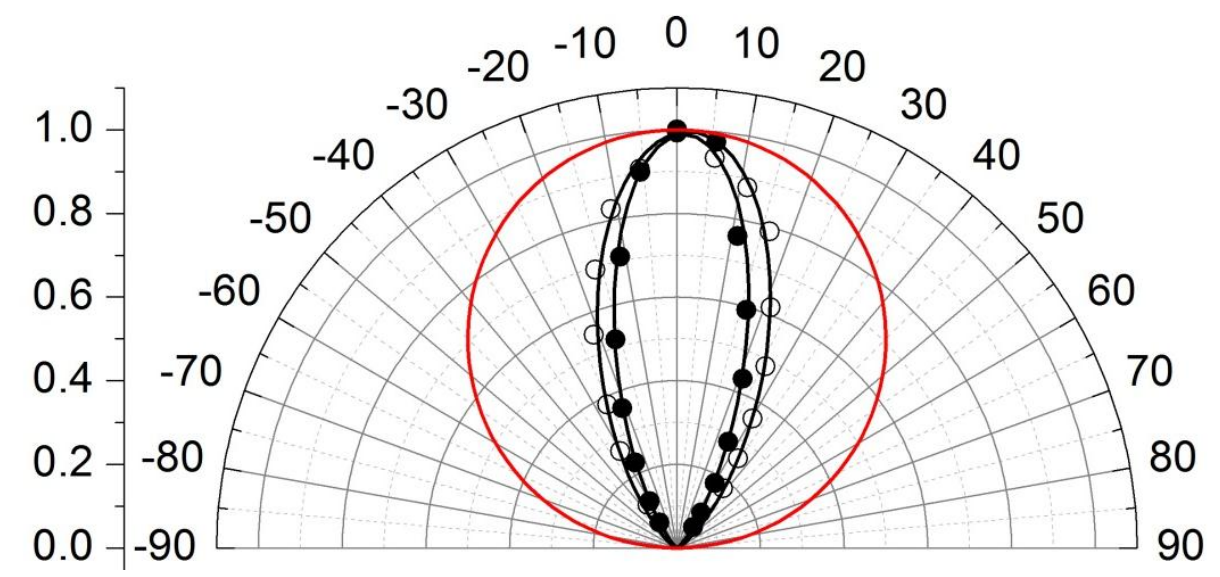

Figure 5.3 Angular distributions are weakly dependent on $\mathrm{T}_{\mathrm{S}}$. Normalized angular distributions are shown for vibrationally excited $\mathrm{NO}(\mathrm{v}=0 \rightarrow 1)$ at two surface temperatures (filled circles, $\mathrm{T}_{\mathrm{S}}=353 \mathrm{~K}$ and empty circles, $\mathrm{T}_{\mathrm{S}}=973$ $\mathrm{K})$ at $\mathrm{E}_{\mathrm{i}}=0.93 \mathrm{eV}$. Gaussian fits to the two angular distributions are shown as black curves. The red line shows a $\cos \theta$ function for comparison, expected if trapping/desorption were important.

Figure 5.4 shows that the angular distributions are not strongly dependent on incidence energy of translational or the final vibrational state. Here, the derived values of $\sigma$ are shown for various scattering experiments.

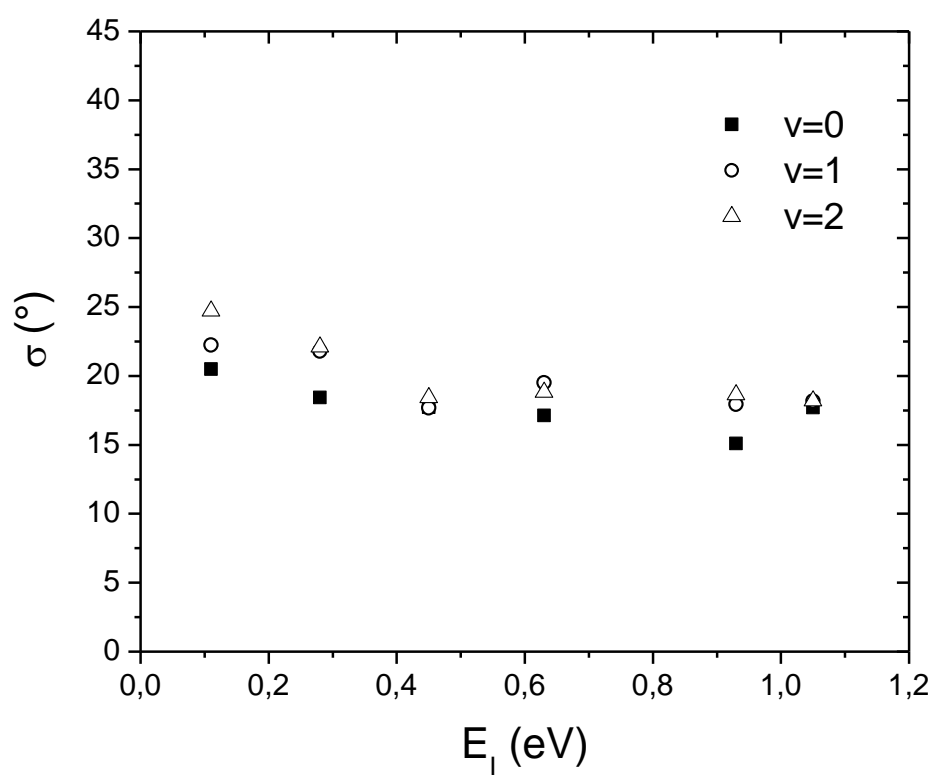

Figure 5.4 The angular distributions are weakly dependent on incidence energy of translation and final vibrational state. Vibrationally elastic scattering $\mathrm{NO}(\mathrm{v}=0 \rightarrow 0)$ filled squares with $\mathrm{T}_{\mathrm{s}}=300 \mathrm{~K}$, as well as vibrationally excited molecules, $\mathrm{NO}(\mathrm{v}=0 \rightarrow 1)$, empty circle, and $\mathrm{NO}(\mathrm{v}=0 \rightarrow 2)$, open triangles at $\mathrm{T}_{\mathrm{s}}=1000 \mathrm{~K}$. 


\subsection{Delay Time Profiles $\Delta_{v}(t)$ :}

The delay time profiles, $\Delta_{\mathrm{V}}(t)$, are used in a similar way as the angular distributions. Here, $\Delta_{\mathrm{V}}(t)$ is normalized numerically and can be used to compensate for differences in the temporal widths of the delay time profiles and for differences in the value of $\tau_{\mathrm{v}}$ chosen by the experimentalist.

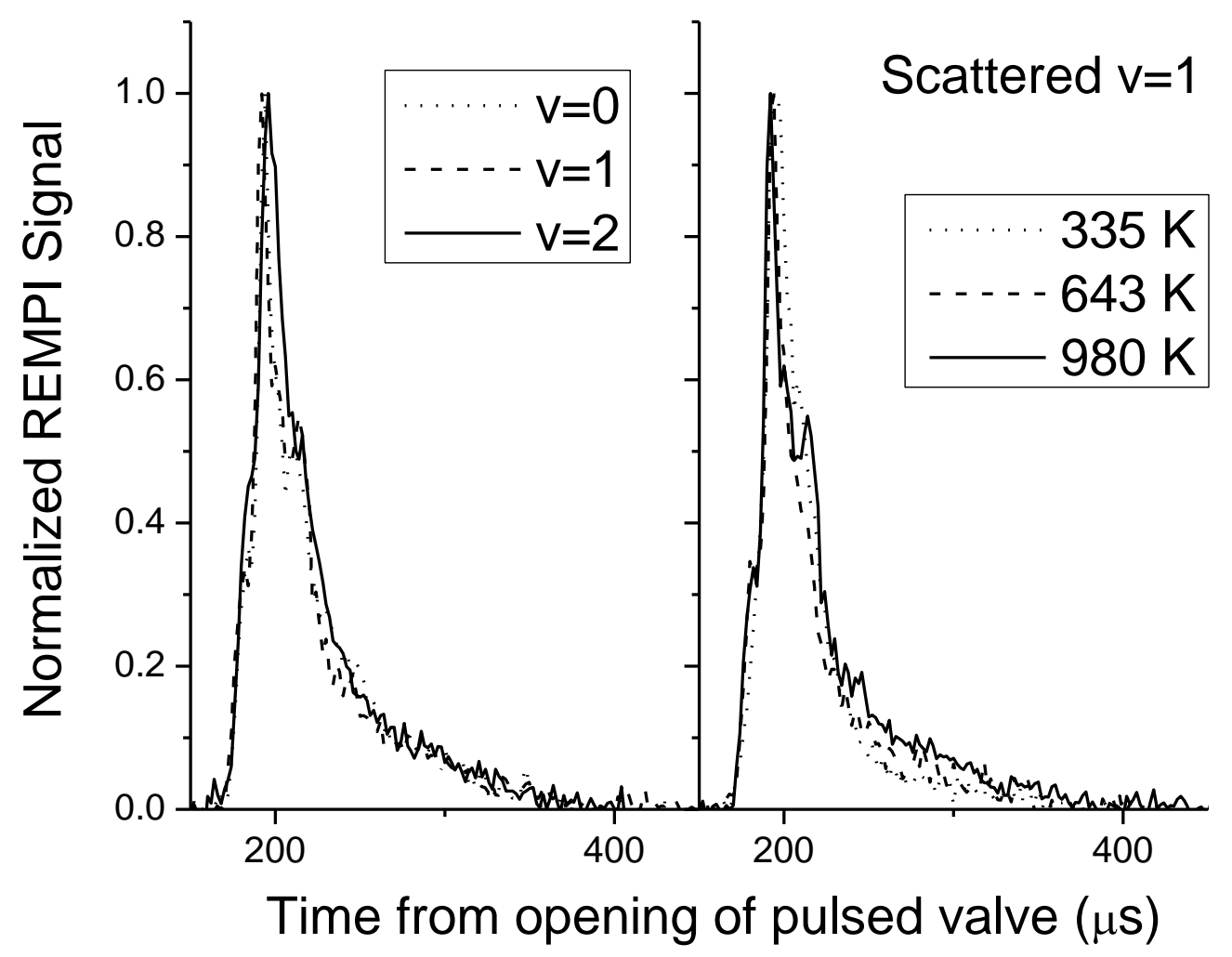

Figure 5.5 Scattered delay time profiles are weakly dependent on final vibrational state and surface temperature. Left panel shows delay time profiles as a function of vibrational state, $\mathrm{NO}(\mathrm{v}=0)$ dot, $\mathrm{NO}(\mathrm{v}=1) \mathrm{dashed}, \mathrm{NO}(\mathrm{v}=2)$ solid at $\mathrm{E}_{\mathrm{i}}=0.93 \mathrm{eV}$. Delay time profiles for $\mathrm{NO}(\mathrm{v}=0)$ occurred at room temperature and $\mathrm{NO}(\mathrm{v}=1)$ and $\mathrm{NO}(\mathrm{v}=2)$ at $\mathrm{T}_{\mathrm{s}}=\sim 1000 \mathrm{~K}$. The right panel shows the effect of surface temperature on scattered $\mathrm{v}=1$ delay time profiles at $\mathrm{E}_{\mathrm{i}}=0.93$ $\mathrm{eV}, \mathrm{T}_{\mathrm{s}}=335 \mathrm{~K}$, dot, $\mathrm{T}_{\mathrm{s}}=643 \mathrm{~K}$, dashed, and $\mathrm{T}_{\mathrm{s}}=980 \mathrm{~K}$, solid.

The delay scan profiles, $\Delta_{\mathrm{V}}(t)$, depend very weakly on the final vibrational state, as shown in the left panel of Fig. 5.5. There is a surface temperature dependence, albeit also weak, on $\Delta_{\mathrm{V}}(t)$, shown in the right panel of Fig. 5.5 for $\Delta_{1}(t)$.

\subsection{Laser power dependence, $f_{\mathrm{v}}\left(I_{\mathrm{v}}\right)$, A-state ionization cross-sections, $\sigma_{\mathrm{v \Sigma}}\left(\lambda_{\text {laser, } \mathrm{v}}\right)$, and MCP Gain, $\Gamma_{\mathrm{v}}$ :}

The magnitude of REMPI signals depends strongly on the laser pulse energy and this is taken into account by finding a suitable empirical function to describe this dependence, $f_{\mathrm{v}}\left(I_{\mathrm{v}}\right)$. For $(1+1)$ REMPI used in this work, we observe a quadratic dependence at low pulse energy which 
becomes linear at higher pulse energies. Although not observed here, we expect REMPI signals to become independent of pulse energy when the laser power is sufficiently high.

$$
f_{\mathrm{V}}\left(I_{\mathrm{V}}\right) \propto\left\{\begin{array}{lr}
I_{\mathrm{V}}{ }^{2} & \text { (at low pulse energy) } \\
I_{\mathrm{V}} & (\text { at intermediate pulse energy) } \\
\text { constant } & \text { (at high pulse energy) }
\end{array}\right.
$$

At low pulse energies neither step of the two-photon absorption process is saturated. At intermediate energies the resonant first photon absorption becomes saturated ${ }^{92-94}$ and at sufficiently high pulse energies both absorptions steps become saturated.

To demonstrate this, I employed a two-laser two-color $\left(1+1^{\prime}\right)$ REMPI approach. Here, a $2 \mathrm{~mm}$ beam of $\sim 230 \mathrm{~nm}$ light from the doubled output of the tunable dye laser, with $0.1-2 \mathrm{~mJ}$ per pulse, was tuned onto a resonance of the $\gamma$-band and coaxially overlapped a $6 \mathrm{~mm}$ beam of $266 \mathrm{~nm}$ light with $24 \mathrm{~mJ}$ per pulse produced from the $4^{\text {th }}$ harmonic of a Nd:YAG laser. Under these conditions, NO is resonantly excited to the $\mathrm{A}^{2} \Sigma^{+}$state by the output of the low power pulse at $\sim 230 \mathrm{~nm}$ and the $\mathrm{A}^{2} \Sigma^{+}$state is nearly exclusively ionized by the $266 \mathrm{~nm}$ pulse. Figure 5.6 shows the dependence of this $\left(1+1^{\prime}\right)$ signal on the energy of the $230 \mathrm{~nm}$ pulse. One can clearly see the saturation of the resonant step of the $\gamma$-band at a pulse-energy of about $1 \mathrm{~mJ}$. In contrast, even with $24 \mathrm{~mJ}$ of power at $266 \mathrm{~nm}$ the ionization step is not saturated.

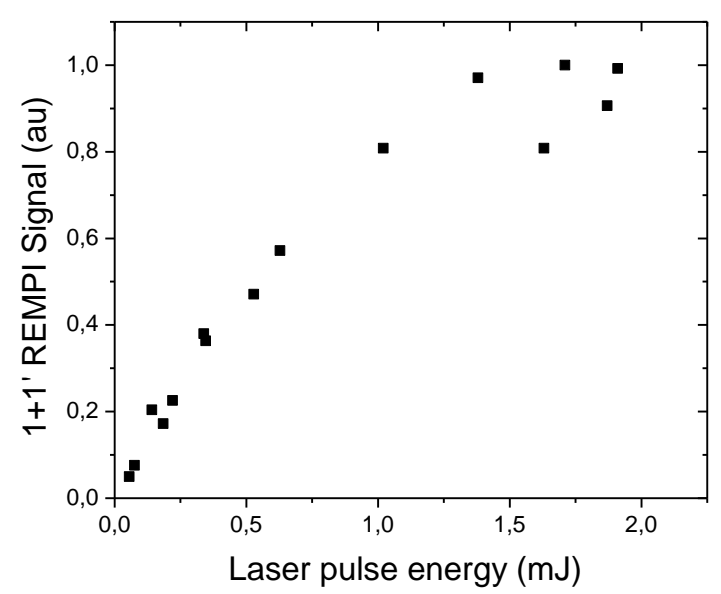

Figure 5.6 Saturation of the resonant step is shown by two color $\left(1+1^{\prime}\right)$ REMPI of scattered $N O(v=1)$. Here the resonant laser pulse energy, 0.1-2 $\mathrm{mJ}$ at $\sim 230 \mathrm{~nm}$ is much weaker than the ionization laser energy, $24 \mathrm{~mJ}$ at $266 \mathrm{~nm}$. The intermediate electronically excited step is predominantly ionized by the $266 \mathrm{~nm}$ light. The resonant laser pulse energy dependence therefore indicates the degree of saturation in the resonant step.

Laser pulse energy dependencies, $f_{\mathrm{v}}\left(I_{\mathrm{v}}\right)$, were measured for different vibrational states, $\mathrm{NO}(\mathrm{v}=0)$, $\mathrm{NO}(\mathrm{v}=1)$, and $\mathrm{NO}(\mathrm{v}=2)$, of the scattered molecules, as well as for $\mathrm{NO}(\mathrm{v}=0)$ in background gas leaked into the chamber. The results of these measurements (Fig. 5.7) are interesting to compare 
to those of Fig. 5.6. As expected one sees a quadratic intensity dependence at the lowest energies which becomes linear above a laser pulse energy of $1 \mathrm{~mJ}$.

Empirically, we find that the laser power dependence can be approximated well by a function $f_{\mathrm{v}}\left(\mathrm{I}_{\mathrm{v}}\right) \propto \mathrm{I}_{\mathrm{v}}{ }^{1.66}$, which is independent of vibrational state.

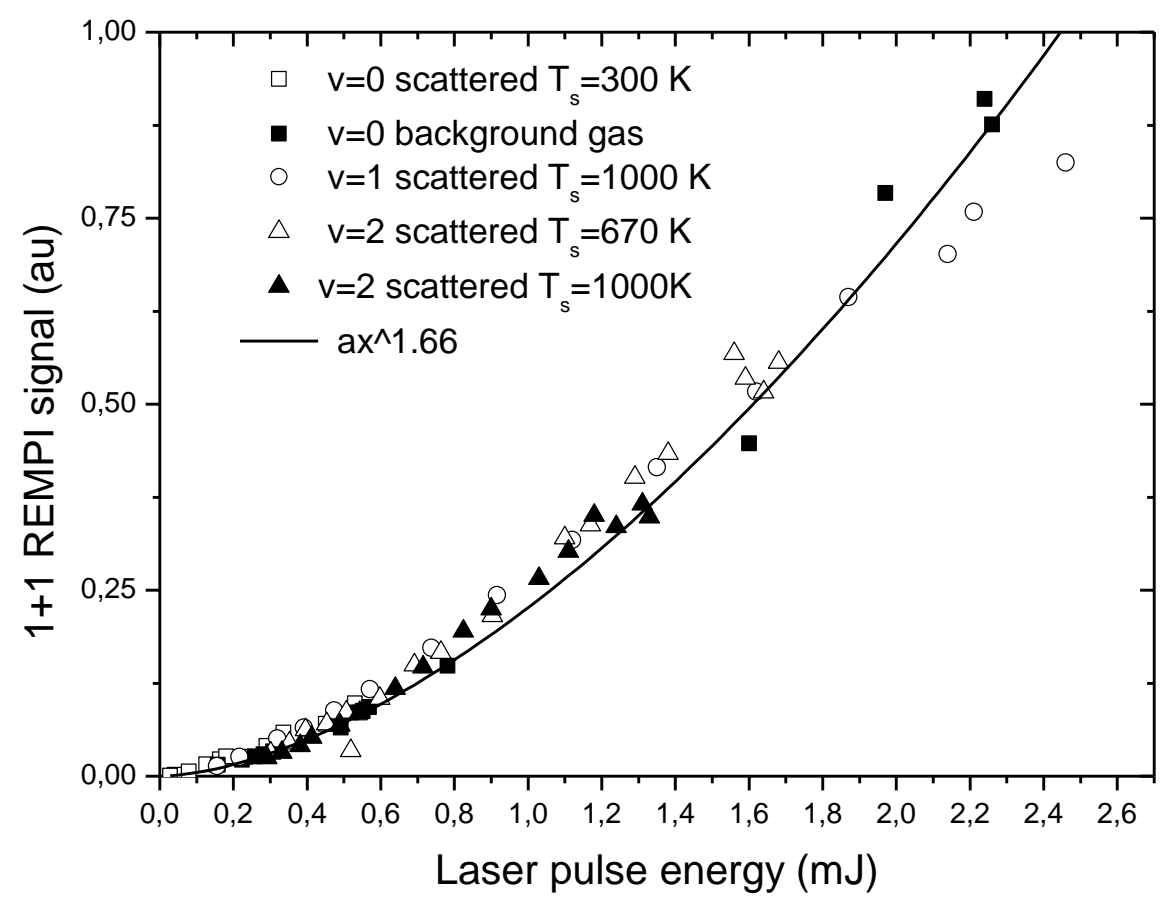

Figure 5.7 Laser energy dependence for $(1+1)$ REMPI ion signal for scattered NO in various vibrational states. Also included is one measurement of background NO leaked into the chamber. The data shows a quadratic laser pulse energy dependence at low laser pulse energies, which becomes linear around $1 \mathrm{~mJ}$. This is indicative that the resonant transition is fully saturated around $1 \mathrm{~mJ}$. The data is well described by the empirical laser pulse energy dependence ax ${ }^{1.66}$, shown as a solid line.

Furthermore, the relative ionization efficiency is nearly identical when probing different vibrational states because the ionization cross section of the $\mathrm{A}^{2} \Sigma^{+}$state is very weakly wavelength dependent between $\lambda_{\text {laser }}=225$ and $324 \mathrm{~nm}^{89,90}$ and all final vibrational states are probed via the $\mathrm{A}^{2} \Sigma^{+}\left(\mathrm{v}_{\Sigma}=0\right)$ state $^{92}$.

Our one-color (1+1) REMPI determinations of absolute vibrational excitation probabilities always employed laser pulse energies substantially above $1 \mathrm{~mJ}$, ensuring saturation of the resonant transition in the $\gamma$-band. This means that the relative ionization efficiencies of different vibrational states are independent of the bands' Franck-Condon factors $Q\left(\mathrm{v}_{\Sigma}, \mathrm{v}\right)$.

The gain of the MCP, $\Gamma_{\mathrm{v}}$, varies as the voltage across the MCP is adjusted to optimize conditions for the detection of each vibrational state. The gain of the MCP is set to a value that allows for a 
reasonable signal-to-noise, while avoiding saturation of the MCP. $\Gamma_{\mathrm{v}}$ was experimentally determined and depending of the voltage difference between $\Gamma_{\mathrm{v}}$ and $\Gamma_{0}, \frac{\Gamma_{\mathrm{v}}}{\Gamma_{0}}$ has a strong effect on $\frac{N_{\mathrm{v}}}{N_{0}}$. The MCP dependence was measured to be:

$$
\Gamma \propto \mathrm{V}^{17.8}
$$

\subsection{The density-to-flux conversion:}

As can be seen in Eq. (5.7) the final velocity of the scattered molecules, $u_{\mathrm{v}}$, is needed to convert density to flux. If the final translational energy were independent of vibrational state, the densityflux conversion would have no influence on the derived excitation probability. It is generally thought that translational to vibrational (T-V) energy transfer is unimportant for vibrationally inelastic NO scattering from $\mathrm{Au}(111)$. As will be shown below, vibrational excitation of $\mathrm{NO}(\mathrm{v}=0 \rightarrow 2)$, with a vibrational energy of $0.46 \mathrm{eV}$, occurs even at incidence energies of translation as low as, $E_{\mathrm{i}}=0.11 \mathrm{eV}$,

Unfortunately, I have little data on the vibrational state dependence of final translational energy. I have investigated the translational energy distributions for two vibrational scattering channels in $\mathrm{NO}(\mathrm{v}=3 \rightarrow 3,2)$ scattering from $\mathrm{Au}(111)$, as shown in section 4.11b. Those measurements show that molecules which undergo vibrational relaxation $\mathrm{NO}(\mathrm{v}=3 \rightarrow 2)$ exhibit an average translational energy that is nearly the same as molecules that were vibrationally elastically scattered $\mathrm{NO}(\mathrm{v}=3 \rightarrow 3)$. Only, $8 \%$ of the vibrational relaxation energy appears as outgoing translational energy. For the following density-to-flux conversion we assume that the vibrationally elastic channel loses a constant fraction of its translational energy, as seen for $\mathrm{HCl}(\mathrm{v}=2) / \mathrm{Au}(111)$ scattering in section 4. Furthermore we assume the $\mathrm{T}-\mathrm{V}$ coupling is the same for vibrational excitation as for relaxation and also independent of the incidence energy of translation. These assumptions allow us to approximately quantify the density-to-flux conversion.

Based on the information presently available to us, I believe that the flux-density conversion is not needed to obtain accurate absolute vibrational excitation probabilities for the vast majority of initial conditions used here. One possible exception is data obtained at the lowest incidence energy of this work, $E_{\mathrm{i}}=0.11 \mathrm{eV}$, where small absolute differences in translation energy can make a significant difference in the final relative velocity and consequently the density-to-flux conversion. We will return to this point in section 5.8, but I re-emphasize that I believe the correction for density-to-flux conversion is smaller than the experimental error, for the vast majority of data reported in this study.

\subsection{Conclusions of the derivation of absolute vibrational excitation probabilities:}

I have now explained how to measure all of the parameters in Eq. (5.7). With this we can derive absolute vibrational excitation probabilities, $P_{0-\mathrm{v}}$ for $\mathrm{NO}(\mathrm{v}=0 \rightarrow 1)$, and $\mathrm{NO}(\mathrm{v}=0 \rightarrow 2)$. For 
$\mathrm{NO}(\mathrm{v}=0 \rightarrow 1)$, background, which arises from rotationally inelastic scattering of thermally populated $\mathrm{NO}(\mathrm{v}=1)$ present in the incident molecular beam, was subtracted. The background level was determined by extrapolating the surface temperature dependence of the $\mathrm{NO}(\mathrm{v}=1)$ signal to $\mathrm{T}_{\mathrm{s}}=0 \mathrm{~K}$ using a suitable Arrhenius expression. This background is small compared to the excitation probability, except when the surface temperature is $300 \mathrm{~K}$

\section{8. $\mathrm{E}_{\mathrm{i}}$ Dependence of NO Vibrational Excitation:}

The derived vibrational excitation probabilities for $\mathrm{NO}(\mathrm{v}=0 \rightarrow 1)$ and $\mathrm{NO}(\mathrm{v}=0 \rightarrow 2)$ are shown in Fig. 5.8. At all incidence energies, the vibrational excitation probabilities follow an Arrhenius dependence on surface temperature with an activation energy equal to the change of the vibrational energy. This is true for both the single and overtone vibrational excitation. This Arrhenius dependence is typical for non-adiabatic vibrational excitation. ${ }^{8,11,12}$ The Arrhenius temperature dependence arises from the distribution of thermally excited electron hole pairs in the surface. ${ }^{8,95,96}$

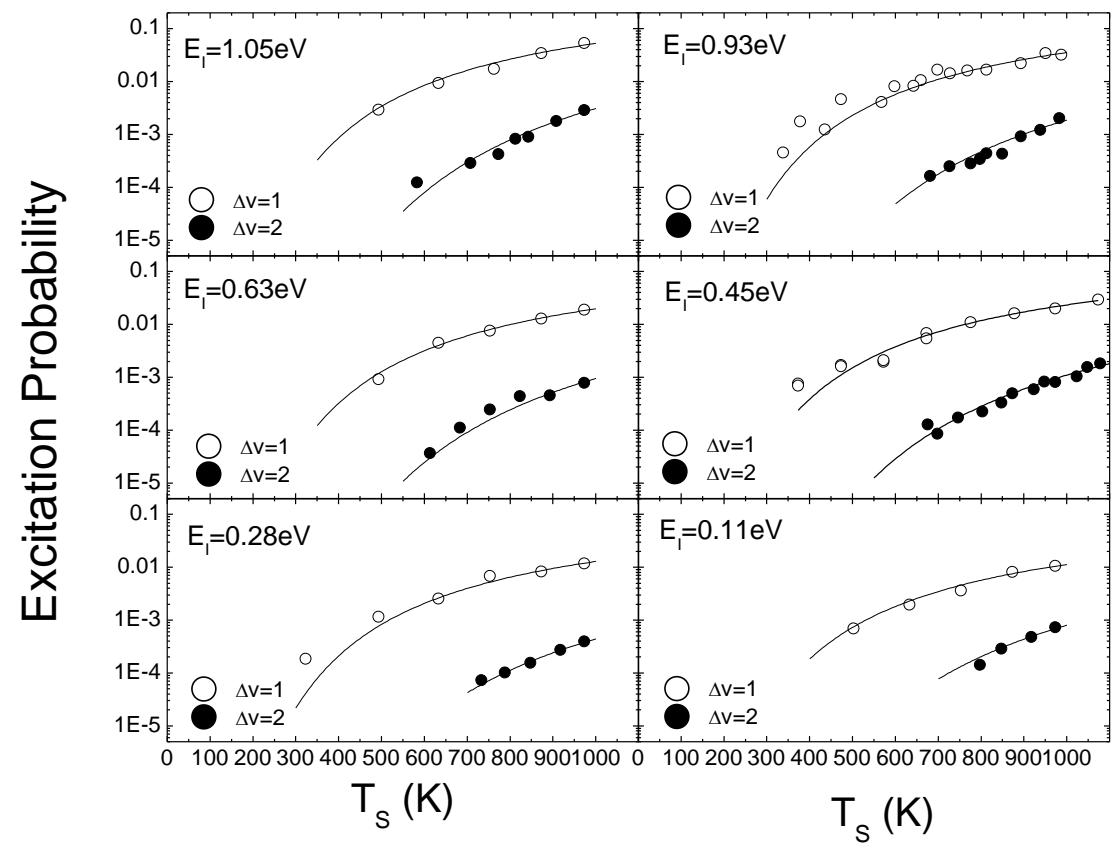

Figure 5.8 Vibrational excitation probabilities for $\mathrm{NO}(\mathrm{v}=0 \rightarrow 1)$, empty circles, and for $\mathrm{NO}(\mathrm{v}=0 \rightarrow 2)$, filled circles, measured as a function of surface temperature for six different incidence energies of translation. The solid lines are Arrhenius fits with the activation energy fixed to the vibrational energy spacing.

In all cases the vibrational excitation is much larger for $\mathrm{NO}(\mathrm{v}=0 \rightarrow 1)$ than for $\mathrm{NO}(\mathrm{v}=0 \rightarrow 2)$. The relative population of the two excited vibrational states tends to not change much as a function of incidence translational energy. The vibrational excitation exhibits an Arrhenius surface temperature dependence at all incidence energies, with the activation energy equal to the vibrational spacing. Additionally, the relative excitation $\mathrm{NO}(\mathrm{v}=0 \rightarrow 1)$ to that of $\mathrm{NO}(\mathrm{v}=0 \rightarrow 2)$ is 
independent of incident translational energy. These facts indicate that over the wide translational energy and surface temperature range used in this study the predominant mechanism for vibrational excitation of $\mathrm{NO}$ scattering from $\mathrm{Au}(111)$ is electronically non-adiabatic coupling. Arrhenius surface temperature dependences have also been seen in theoretical studies that do not include electron hole pair coupling. ${ }^{21,97}$ However, it is likely that with the short interaction time, of a direct scattering event, and the mismatch in energy between phonons in gold (a Debye energy of $\left.118 \mathrm{~cm}^{-1}\right)$ and the vibrational energy in $\mathrm{NO}(\mathrm{v}=1)\left(1903 \mathrm{~cm}^{-1}\right)$ or $\mathrm{NO}(\mathrm{v}=2)\left(3806 \mathrm{~cm}^{-1}\right)$ coupling energy transfer between the molecule and phonons would not lead to substantial vibrational excitation.

In order to have a clear picture of how vibrational excitation is influenced by the incidence energy of translation, I derive an Arrhenius pre-factor for every data point with the assumption that the Arrhenius activation energy equals the vibrational energy spacing. The Arrhenius prefactor is useful as it indicates the strength of the vibrational excitation. ${ }^{96}$ This will be discussed in more detail in section 6. These pre-factors, averaged for different temperatures, are plotted versus incidence translational energy in Fig. 5.9. Error for the averaged pre-factor, with a $90 \%$ confidence interval, was calculated according to the Student's t-test. Here the results for the room temperature surface for $\mathrm{NO}(\mathrm{v}=0 \rightarrow 1)$ were removed, as at low surface temperatures the excitation probability is as small or smaller than that of the $\mathrm{NO}(\mathrm{v}=1)$ native in the incident beam making the derived value of the vibrational excitation of molecules scattered from a room temperature surface quite unreliable.

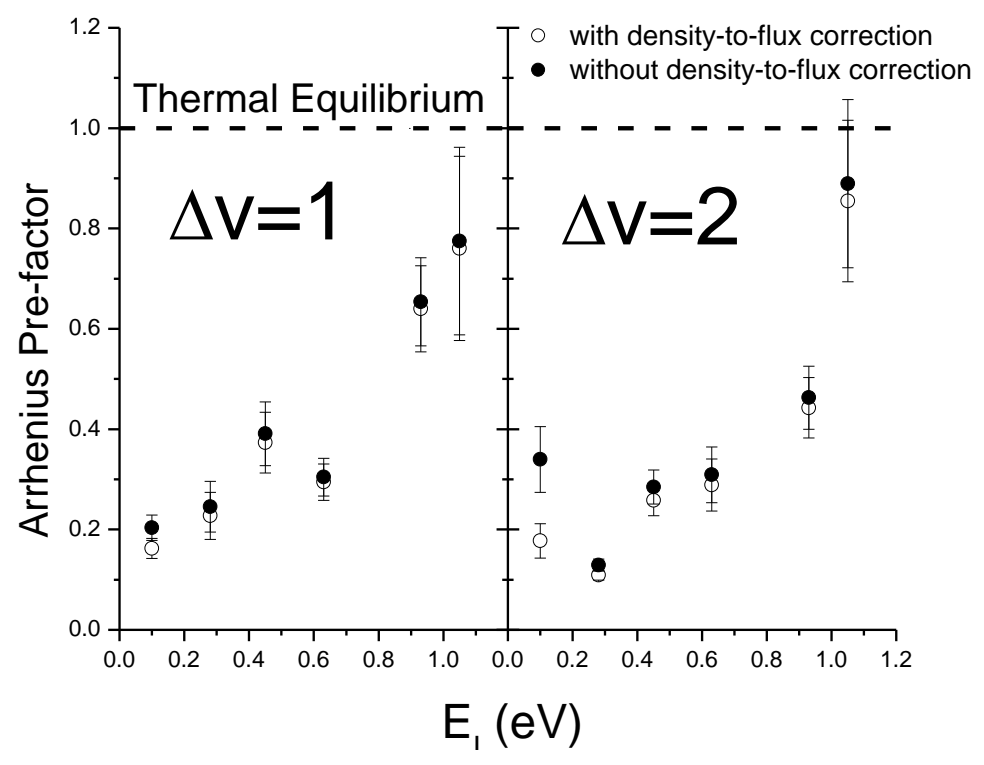

Figure 5.9 Arrhenius pre-factor with, empty circles, and without, filled circles, density to flux conversion plotted as a function of incidence energy of translation for $\mathrm{NO}(\mathrm{v}=0 \rightarrow 1)$, left panel and $\mathrm{NO}(\mathrm{v}=0 \rightarrow 2)$, right panel. The error bars represent the $90 \%$ confidence interval. The dashed line represents the thermal equilibrium value of the Arrhenius pre-factor, 1 . See text. 
As has been previously seen there is a strong incidence energy of translation dependence in nonadiabatic vibrational excitation. ${ }^{8,29}$ The strength of the interaction of the vibrational and electronic motion can be characterized by looking at the magnitude of the Arrhenius pre-factors. A pre-factor of " 1 " indicates that the vibrational degree of freedom has been brought into equilibrium with the temperature of the surface. A pre-factor of " 0 " means no vibrational excitation took place. ${ }^{96}$ Further details are given in section 6 . In direct scattering, pre-exponential values close to one, such as those seen here, represent strong coupling between the molecular vibration and the electrons in the metal. In contrast to the strong coupling seen in this thesis, the vibrational excitation of $\mathrm{HCl}(\mathrm{v}=0 \rightarrow 1)$ when scattered from $\mathrm{Au}(111)$ is much weaker. ${ }^{12,18}$ The pre-factors for this vibrational excitation was more than two orders of magnitude smaller than those seen here.

The average ratio for the Arrhenius pre-factors of $\mathrm{NO}(\mathrm{v}=0 \rightarrow 1)$ and $\mathrm{NO}(\mathrm{v}=0 \rightarrow 2)$ at all incidence energies is 1.2. This shows that the direct $\mathrm{NO}(\mathrm{v}=0 \rightarrow 2)$ coupling is nearly as strong as that for $\mathrm{NO}(\mathrm{v}=0 \rightarrow 1)$. It is likely that within the spread of pre-factors derived in this work, the coupling strengths for $\mathrm{NO}(\mathrm{v}=0 \rightarrow 1)$ and overtone $\mathrm{NO}(\mathrm{v}=0 \rightarrow 2)$, do not differ significantly. This is strong evidence that vibrational excitation of $\mathrm{NO}(\mathrm{v}=2)$ occurs predominantly via direct $\mathrm{NO}(\mathrm{v}=0 \rightarrow 2)$ overtone and not sequential $\mathrm{NO}(\mathrm{v}=0 \rightarrow 1 \rightarrow 2)$.

One intriguing feature of the incidence translational energy dependence is that linear extrapolation of the incidence translational energy dependence to zero gives a non-zero excitation probability for both $\mathrm{NO}(\mathrm{v}=0 \rightarrow 1)$ and $\mathrm{NO}(\mathrm{v}=0 \rightarrow 2)$. At $\mathrm{E}_{\mathrm{i}}=0.11 \mathrm{eV}$ the density-to-flux conversion can have a large effect on the derived Arrhenius pre-factor as small amounts of T-V coupling significantly alter the ratio in final translational energies of the vibrationally elastic $\mathrm{NO}(\mathrm{v}=0 \rightarrow 0)$ channel and the inelastic channels $\mathrm{NO}(\mathrm{v}=0 \rightarrow 1,2)$. Figure 5.9 shows the derived vibrational excitation probabilities both with density-to-flux corrections, empty circles, and without density-to-flux corrections, filled circles. Under most of the conditions in this work the difference in the derived vibrational excitation probabilities by incorporating density to flux or not is $<10 \%$. The one exception is the case of $\mathrm{E}_{\mathrm{i}}=0.11 \mathrm{eV}$, where, especially for the $\mathrm{NO}(\mathrm{v}=0 \rightarrow 2)$ channel, there is a large density-to-flux correction. A better study of the translational energy is needed to make a definitive statement about the size of the density-to-flux correction at $\mathrm{E}_{\mathrm{i}}=0.11$ $\mathrm{eV}$ and consequently the magnitude of the pre-exponential factors for this energy.

Trapping desorption (TD), where the molecules trap temporarily on the surface and then desorb, may also influence the results at $\mathrm{E}_{\mathrm{i}}=0.11 \mathrm{eV}$. The trapping probability of $\mathrm{NO}$ scattering from $\mathrm{Au}$ was previously measured and was found to be near 0.40 at $E_{\mathrm{i}}=0.11 \mathrm{eV}{ }^{60}$ Estimates for the residence time can be made by using the equation $10^{-12} * e^{\left(E_{a} /\left(k_{b} T\right)\right)}$ where $E_{a}$ is the binding energy of the NO on the $\mathrm{Au}(111)$ surface. The binding energy of $\mathrm{NO} / \mathrm{Au}(111)$ was calculated to be 0.13 $\mathrm{eV}^{40}$ While this quantity has not been determined experimentally, experimental evidence indicates that the calculated value is approximately correct. ${ }^{60,98}$ For the binding energy, $0.13 \mathrm{eV}$, the residence time for $\mathrm{NO}$ on $\mathrm{Au}(111)$ at $\mathrm{T}_{\mathrm{s}}=798$ and $1000 \mathrm{~K}$, ranges from $6.6^{*} 10^{-12}$ to $4.5^{*} 10^{-12}$ 
s, substantially longer than the interaction time with the surface in direct scattering, $\sim 400 \mathrm{fs}$. The increased residence time allows for a longer interaction and consequently for stronger vibrational excitation. The actual effect of trapping on vibrational excitation is further complicated by the orientation dependence of both trapping and direct vibrational excitation. Theory predicts that for NO interactions with $\mathrm{Au}(111)$ the coupling of vibrational and electronic degrees of freedom is stronger for collisions of the molecule with the $\mathrm{N}$-end oriented towards the surface. ${ }^{42}$ Trapping for $\mathrm{NO}$ on $\mathrm{Ag}(111)$, a very similar system, also is enhanced for the the O-end towards the surface. ${ }^{99}$ How these factors would combine to effect the vibrational excitation probabilities is hard to predict. Further theoretical and experimental work is needed to fully understand this interplay.

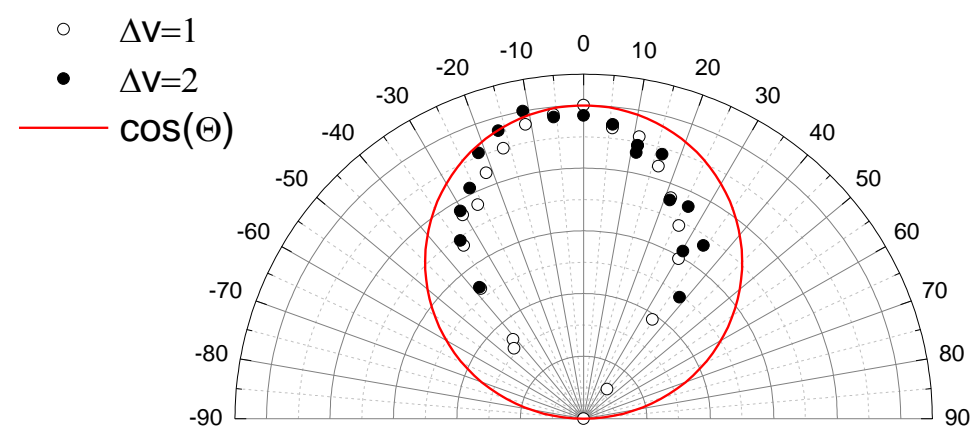

Figure 5.10 Angular distributions for $\mathrm{NO}(\mathrm{v}=0 \rightarrow 1)$, empty circles, and $\mathrm{NO}(\mathrm{v}=0 \rightarrow 2)$, solid circles for $\mathrm{E}_{\mathrm{i}}=0.11 \mathrm{eV}$ and $\mathrm{T}_{\mathrm{s}}=1000 \mathrm{~K} \cdot \operatorname{Cos}(\theta)$ is plotted in red.

The angular distribution for both $\mathrm{NO}(\mathrm{v}=0 \rightarrow 1)$ and $\mathrm{NO}(\mathrm{v}=0 \rightarrow 2)$ at $\mathrm{E}_{\mathrm{i}}=0.11 \mathrm{eV}$ is quite broad, approaching the width of the $\cos (\theta)$ distribution expected from trapping desorption. However this alone does not indicate that trapping desorption is the predominant mechanism because as the incidence kinetic energy is lowered, the energy of a hot surface $(1000 \mathrm{~K}=\sim 0.9 \mathrm{eV})$ becomes comparable to the incident translational energy allowing for direct scattering to become quite broad in final scattering angle.

An argument against TD playing a prominent role is that the rotational temperatures, discussed in detail later, at $\mathrm{E}_{\mathrm{i}}=0.11 \mathrm{eV}\left(\mathrm{NO}(\mathrm{v}=1)\right.$ average $\left.\mathrm{T}_{\mathrm{R}}=403 \mathrm{~K}\right)$ are well below the surface temperature, and are also lower than those seen at $E_{i}=0.28 \mathrm{eV}\left(\mathrm{NO}(\mathrm{v}=1)\right.$ average $\left.\mathrm{T}_{\mathrm{R}}=432 \mathrm{~K}\right)$, where trapping is not thought to occur. This would seem to show that most molecules do not undergo TD at the surface. 
Dynamical steering of the incoming molecules could also lead to the observation of larger excitation probabilities at low incidence energies. Previous theoretical work has shown that NO scattering with $\mathrm{N}$ leading, provides better overlap with the electrons in the metal, which consequently leads to increased vibrational excitation and that the $\mathrm{NO} \mathrm{Au}(111)$ interaction tends to steer the molecules into strong coupling orientations. ${ }^{42}$ At small incidence energies of translation the molecules move slower allowing for more extensive orientation of the molecules. This provides an additional possibility for the increased vibrational excitation at $\mathrm{E}_{\mathrm{i}}=0.11 \mathrm{eV}$.

\section{9 $\mathrm{E}_{\mathrm{i}}$ and $\mathrm{T}_{\mathrm{S}}$ dependence of Rotational Excitation:}

Full REMPI spectra probing the entire rotational distribution in each of the scattered vibrational states were recorded. These rotational spectra examples of which are shown in Fig. 5.1 have been fit, using LIFBase, ${ }^{100}$ to a rotational temperature. This allows a simple way to compare the extent of rotational excitation of the molecules upon scattering from the surface.

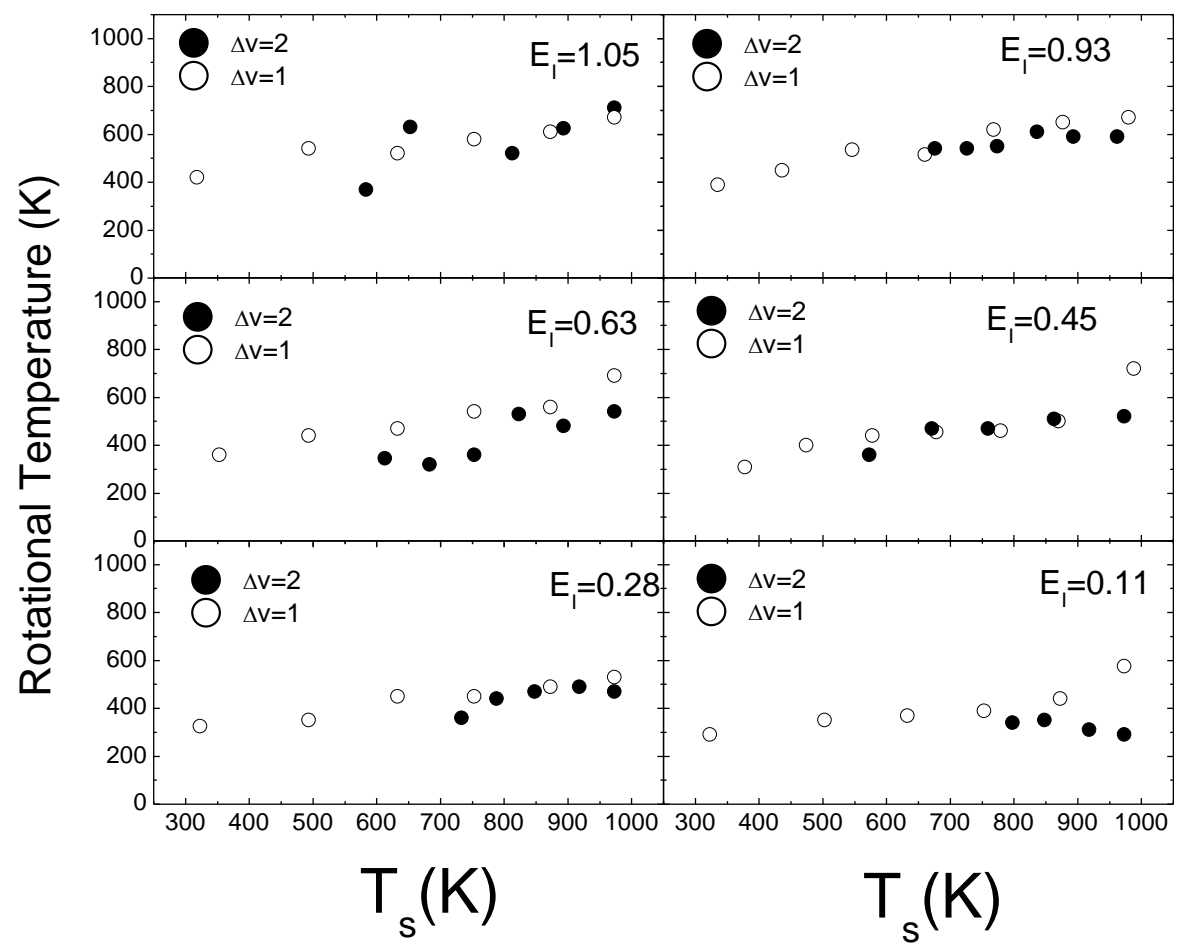

Figure 5.11 Rotational temperature of $\mathrm{NO}(\mathrm{v}=0 \rightarrow 1)$, empty circles, and $\mathrm{NO}(\mathrm{v}=0 \rightarrow 2)$, filled circles, as a function of surface temperature at six different incidence translational energies.

The rotational excitation of the scattered molecules does not depend strongly on the final vibrational state. The rotational excitation increases when surface temperature or incidence energy of translation is increased. The rotational temperature, except by coincidence, is different than the temperature of the surface. These trends in rotational excitation are qualitatively similar to NO scattering from $\operatorname{Ag}(111){ }^{64}$ 
In this section, I explained how to derive absolute excitation probabilities. I presented results of vibrational excitation for multiple surface temperatures and incidence energies of translation. An average Arrhenius pre-factor, at all surface temperatures, was derived for each incidence translational energy. The dependence of the derived Arrhenius pre-factor on the incidence energy of translation was discussed. Perhaps of particular note was that extrapolation to zero kinetic energy did not result in a zero vibrational excitation probability. Effects of trapping and orientation on absolute excitation probabilities were also discussed. 


\section{Kinetic model for NO vibrational excitation:}

In essence, hopping of electrons from the metal surface to the lowest unoccupied molecular orbital (LUMO) of the molecule, which becomes energetically accessible at some critical molecule-surface distance is the mechanism of energy transfer in electronically nonadiabatic vibrational excitation. At this distance, the molecular affinity level $|a\rangle$ with energy $\varepsilon_{a}(z)$ approaches the energy of the Fermi level due to Coulomb image charge stabilization and is also broadened as depicted in Fig. 6.1.

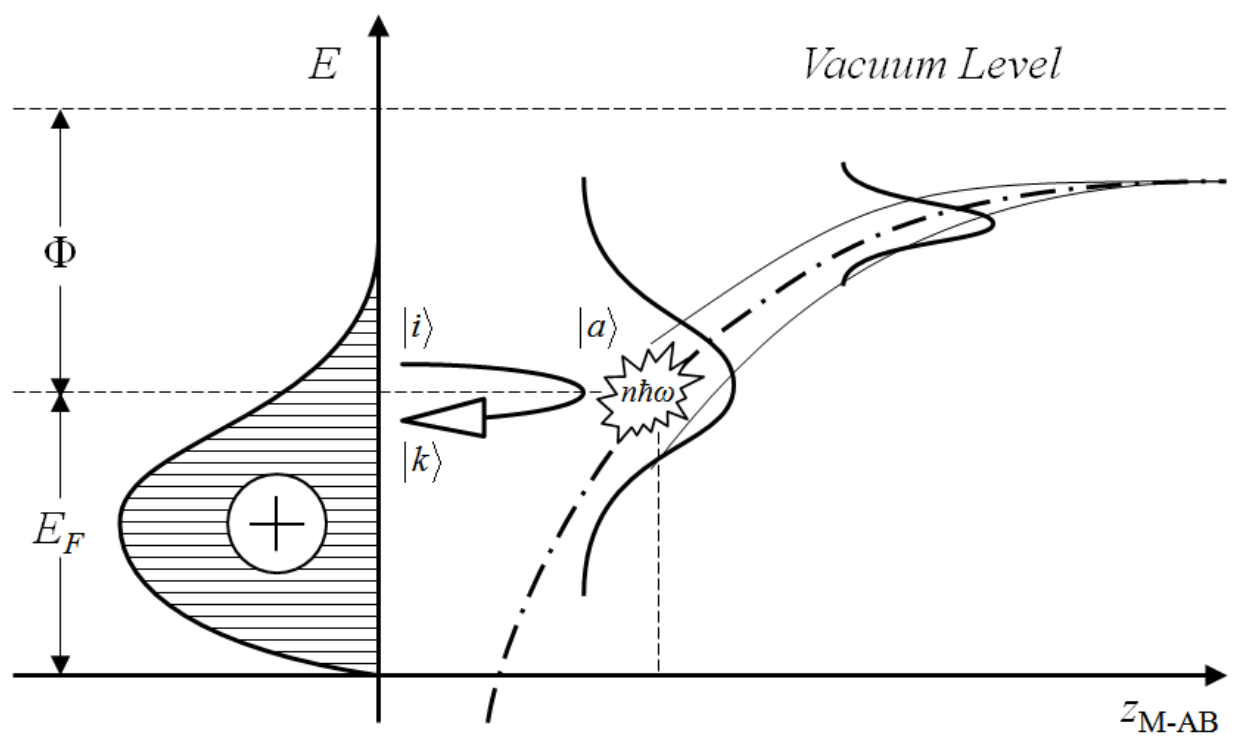

Figure 6.1 Energy as a function of molecule-surface distance. Hatched area depicts conduction electrons and the positive image charge as the product of the Fermi-Dirac function and the three-dimensional density of states; $\mathrm{E}_{\mathrm{F}}$ is the Fermi level; $\Phi$ is the work function of the metal surface; $|a\rangle$ is the stabilized and broadened affinity level; electron $|i\rangle$ and $|k\rangle$ are the initial and final electronic states in the metal. ${ }^{96}$

When $\mid a>$ is energetically near the Fermi level, the molecule can form a short-lived anionic state by resonantly accommodating an electron from the surface into the LUMO. The rapid return of the electron to the metal results in lifetime broadening of $|a\rangle$, which decreases with distance from the surface. The molecule's vibrational degree of freedom can either be excited or relaxed depending on the precise energies of the initial $|i\rangle$ and final $\mid k>$ states of the exchanged electron in the metal.

In understanding the excitation probabilities for $\mathrm{NO}(\mathrm{v}=0 \rightarrow 1,2)$, a kinetic model was developed that allowed for additional insights to be drawn from the dependence of vibrational excitation on surface temperature. In this section, the temperature dependence on vibrational excitation from a single incidence translational energy, $\mathrm{E}_{\mathrm{i}}=0.93 \mathrm{eV}$, shown in section 6 will be used. The temperature dependence for vibrational excitation of molecules colliding with metal surfaces, when the vibrational excitation mechanism involves coupling to electron hole pairs, commonly 
follows an Arrhenius like dependence on the surface temperature, where the activation energy is set to the vibrational energy change:

$$
P\left(\Delta E_{v i b}\right)=A e^{-\frac{\Delta E_{v i b}}{k_{b} T_{s}}}
$$

Here, $P$ is the excitation probability, $\Delta \mathrm{E}_{\text {vib }}$ is the vibrational excitation energy, $\mathrm{T}_{\mathrm{S}}$ is the surface temperature, and $\mathrm{k}_{\mathrm{B}}$ is the Boltzmann constant. The pre-factor, A, reflects the intrinsic coupling between EHPs and molecular vibration while the exponential term expresses, albeit in an approximate fashion, the statistical likelihood to find a thermally excited EHP of the correct energy to excite molecular vibration. Experimentally determining state-to-state pre-factors is one possible way to obtain the relative importance of vibrational overtone excitation, as the prefactors can give some information about the strength of the coupling, independent of the electronic statistics of the metal surface. There are a few limitations of Eq. (6.1) however. For example, as the coupling becomes very strong, or the temperature very high, Eq. (6.1) breaks down as it will yield values higher than that obtained if the NO vibrational come to thermal equilibrium with the surface. This is perhaps most obvious at very high surface temperatures where the excitation probability could approach unity, which is physically unreasonable.

It is a subtle matter to use the Arrhenius equation to distinguish between direct and sequential vibrational excitation. The expectation for the surface temperature dependence for direct $\mathrm{NO}(\mathrm{v}=0 \rightarrow 2)$ and sequential $\mathrm{NO}(\mathrm{v}=0 \rightarrow 1 \rightarrow 2)$ excitation are shown below:

$$
P_{\text {direct }}(0-2)=A_{0,2} e^{-\frac{2 \mathrm{~h} v}{\mathrm{k}_{\mathrm{B}} \mathrm{T}}}
$$

$$
\begin{aligned}
& P_{\text {sequential }}(0-2)=P_{\text {direct }}(0-1) * P_{\text {direct }}(1-2)=A_{0,1} e^{-\frac{\mathrm{h} v}{\mathrm{k}_{\mathrm{B}} \mathrm{TS}}} A_{1,2} e^{-\frac{\mathrm{h} v}{\mathrm{k}_{\mathrm{B}} \mathrm{T}_{\mathrm{S}}}}= \\
& A_{0,1} A_{1,2} e^{-\frac{2 \mathrm{~h} v}{\mathrm{k}_{\mathrm{B}} \mathrm{TS}}}
\end{aligned}
$$

Here the final result differs only in the pre-exponential of the Arrhenius equation. In other words, it is the absolute value of excitation efficiency and not the temperature dependence that holds the answer to distinguishing direct from sequential multi-quantum excitation.

To make these matters easier to understand, I developed and implemented a kinetic model for vibrational state changing events occurring in collisions with a metal surface. This model incorporates microscopic reversibility and a statistical treatment of the thermally excited metallic EHPs modeled as a Fermi gas of electrons in a three dimensional box. The model includes rate processes that describe one and two quantum level changes between the three lowest vibrational states: $v=0,1$, and 2. In addition to describing deviations from Eq. (6.1) associated with the transition to equilibrium with the surface, and deviations which occur at high surface temperatures, the model also helps unravel the mechanism for multi-quantum vibrational 
excitation. These rate equations were solved both numerically and analytically. The numerical solution provides simpler calculations, however they mask somewhat a clear analysis of the temperature dependence of the vibrational excitation. For a more detailed look into the kinetic model, the analytical solutions should be used. It is important to note that for more complicated systems such as $\mathrm{NO}(\mathrm{v}=18)$ relaxation from $\mathrm{Au}(111)$, or to have time dependent rate constants, as necessary for modeling the incidence energy of translation dependence, the numerical solutions cost much less in computational time. Here an analysis with numerical solutions should be undertaken.

I use a simple rate equation method to describe EHP mediated energy transfer. The model includes six rate processes:

$$
\begin{aligned}
& n_{0} \rightleftharpoons n_{1} \\
& n_{0} \rightleftharpoons n_{2} \\
& n_{1} \rightleftharpoons n_{2}
\end{aligned}
$$

with six rate constants: three for vibrational excitation:

$$
\begin{aligned}
& k_{0,1}=\xi_{0,1} * f_{1}\left(T_{S}\right) \\
& k_{1,2}=\xi_{1,2} * f_{1}\left(T_{S}\right) \\
& k_{0,2}=\xi_{0,2} * f_{2}\left(T_{S}\right)
\end{aligned}
$$

and three for vibrational de-excitation:

$$
\begin{aligned}
& k_{1,0}=\xi_{1,0} * f_{-1}\left(T_{S}\right) \\
& k_{2,1}=\xi_{2,1} * f_{-1}\left(T_{S}\right) \\
& k_{2,0}=\xi_{2,0} * f_{-2}\left(T_{S}\right)
\end{aligned}
$$

Here, $n_{i}$ is the population of the $\mathrm{i}^{\text {th }}$ vibrational state and $k_{i, j}$ is the rate constant for transitions from state $i$ to state $j$. Each rate process is modeled as a product of an intrinsic coupling strength, $\xi_{i, j}$ and an electronic statistical factor, $f_{\Delta v}\left(T_{S}\right)$ of the surface, which I will presently define. Microscopic reversibility states that $\xi_{i, j}=\xi_{j, i}$.

The electronic statistics are calculated as follows. I first assume that the Fermi function:

$$
\mathrm{F}\left(\varepsilon ; \mathrm{T}_{\mathrm{S}}\right)=\frac{1}{1+e^{\frac{\varepsilon-\varepsilon}{\mathrm{k}_{\mathrm{B}} \mathrm{S}}}}
$$

when combined with the density of states function of a spin- $1 / 2$ particle in a three dimensional box: 


$$
\rho(\varepsilon)=2 \times 4 \pi \sqrt{\frac{2 m_{e}^{3}}{h^{6}}} V \sqrt{\varepsilon}
$$

adequately describes the energy distribution of the electrons in the metal near the Fermi level. Here, $\varepsilon_{\mathrm{F}}$ is the Fermi energy; $m_{e}$ is the electron mass; $h$ is Planck's constant and $V$ is the volume of the metal. The electron distribution is then given by,

$$
p_{e}\left(\varepsilon ; \mathrm{T}_{\mathrm{S}}\right)=\mathrm{F}\left(\varepsilon, \mathrm{T}_{\mathrm{S}}\right) * \rho(\varepsilon)
$$

while the hole distribution is:

$$
p_{h}\left(\varepsilon ; \mathrm{T}_{\mathrm{S}}\right)=\rho(\varepsilon) *\left(1-\mathrm{F}\left(\varepsilon, \mathrm{T}_{\mathrm{S}}\right)\right)
$$

Using these distributions I define:

$f_{\Delta v}\left(T_{S}\right)=\int_{\varepsilon=0}^{\infty} \mathrm{d} \varepsilon \int_{\varepsilon^{\prime}=0}^{\infty} \mathrm{d} \varepsilon^{\prime} p_{e}\left(\varepsilon ; \mathrm{T}_{\mathrm{S}}\right) * p_{h}\left(\varepsilon^{\prime} ; \mathrm{T}_{\mathrm{S}}\right) * \delta\left(\varepsilon-\varepsilon^{\prime}-\Delta v * \mathrm{~h} v\right)$

where $\Delta v$ are the integers \pm 1 or \pm 2 and combined with $h v$ gives the vibrational energy change of the molecule and thus the required energy of the electron hole pair.

The function $f_{\Delta v}\left(T_{S}\right)$ represents the sum over all states where a thermally excited EHP is present whose relaxation energy is equal to the vibrational excitation, $\Delta v>0$. In a similar fashion, it can also represent the sum over all electronic states where an electron and a higher energy hole are capable of accepting the vibrational energy, $\Delta v<0$. With these definitions of the rate constants, the system of differential equations is solved analytically using the Laplace Transform method, assuming initial conditions where population is found only in $v=0$.

$$
\begin{aligned}
& \frac{d n_{0}(t)}{d t}=-\left(\mathrm{k}_{0,1}+\mathrm{k}_{0,2}\right) n_{0}(\mathrm{t})+\mathrm{k}_{1,0} n_{1}(\mathrm{t})+\mathrm{k}_{2,0} n_{2}(\mathrm{t}) \\
& \frac{d n_{1}(t)}{d t}=\mathrm{k}_{0,1} n_{0}(\mathrm{t})-\left(\mathrm{k}_{1,0}+\mathrm{k}_{1,2}\right) n_{1}(\mathrm{t})+\mathrm{k}_{2,1} n_{2}(\mathrm{t}) \\
& \frac{d n_{2}(t)}{d t}=\mathrm{k}_{0,2} n_{0}(\mathrm{t})+\mathrm{k}_{1,2} n_{1}(\mathrm{t})-\left(\mathrm{k}_{2,0}+\mathrm{k}_{2,1}\right) n_{2}(\mathrm{t})
\end{aligned}
$$

where:

$$
n_{0}(0)=1 \text { and } n_{1}(0)=n_{2}(0)=0
$$

It is useful to envision a physical picture to accompany the mathematical solutions obtained from the model; perhaps most importantly this helps illuminate the assumptions of the model. Once can envision a molecule colliding with a surface in a direct scattering process. The model assumes that non-adiabatic coupling occurs with a constant magnitude. Thus as the molecule approaches the surface, there is some distance where the coupling turns on like a step function. It is then further assumed that the coupling remains constant during an effective collision time, $\tau_{\text {eff. }}$ One may note from this comparison that when we arbitrarily fix $\xi_{0,1}$, we may vary the population 
transfer by adjusting $\tau_{\text {eff }}$, or vise-versa. However in all of the comparisons with experimental data that we will make below, $\xi_{0,1}=1$. I then adjust the effective collision time, $\tau_{\text {eff, }}$ to describe the overall strength of coupling. I can then vary the magnitudes of $\xi_{1,2}$ and $\xi_{0,2}$ with respect to $\xi_{0,1}$ to explore the relative importance of sequential and multi-quantum processes

\subsection{Comparison to data:}

In this section, I compare the kinetic model to the experimentally derived vibrational excitation probabilities of $\mathrm{NO}$ scattered from a $\mathrm{Au}(111)$ surface with an $\mathrm{E}_{\mathrm{i}}=0.93 \mathrm{eV}$. Although there are many adjustable parameters in the kinetic model, $\tau_{\text {eff }}, \xi_{0,1}, \xi_{1,2}$ and $\xi_{0,2}$, we easily find a robust fit to the data. In this fitting procedure, $\xi_{0,1}$ is arbitrarily set to one and $\tau_{\text {eff }}$ is allowed to vary as previously described. Fits of the data are only weakly dependent on the strength of $\xi_{1,2}$. Thus in practice, only $\tau_{\text {eff }}$ and $\xi_{0,2}$ strongly affect the quality of the fit. These two fitting parameters have very different effects on the data, $\tau_{\text {eff }}$ controls the overall magnitude of the coupling for vibrational excitation and $\xi_{0,2}$ the relative population of $\mathrm{NO}(\mathrm{v}=2)$ to $\mathrm{NO}(\mathrm{v}=1)$.

Figure 6.2 shows the best fit to the data. Comparing to the thermal limit, dot-dashed curves, noting the derived value of $\tau_{\text {eff }}=1.46$, and taking into account that the scattering occurs in a short time, the electronically non-adiabatic coupling is clearly strong. The value of $\tau_{\text {eff }}$ represents a coupling strength that brings the system roughly half way to the thermal limit.

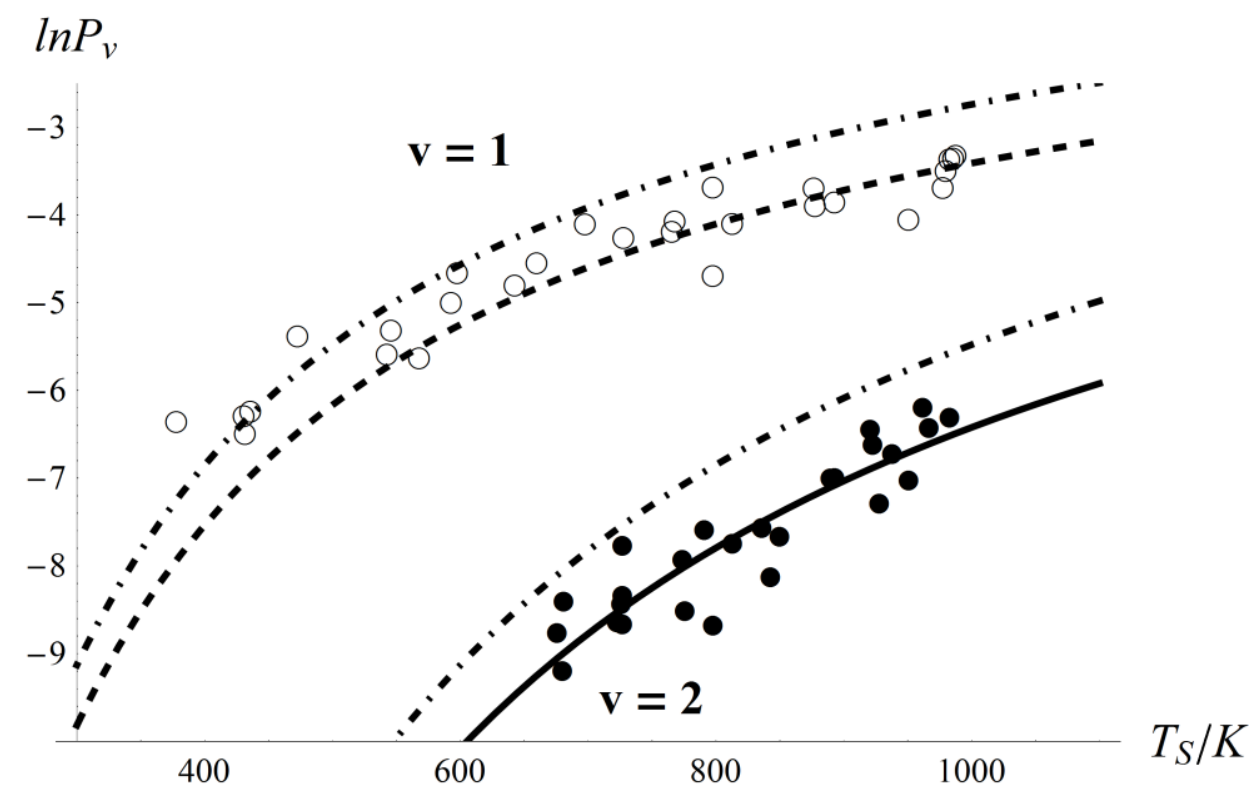

Figure 6.2 Comparison of experimental NO vibrational excitation probabilities, $\mathrm{P}_{\mathrm{v}}$, to the kinetic model. The effective collision time was $\tau_{\text {eff }}=1.46$, and the state-to-state coupling was $\xi_{0,2}=0.30 * \xi_{0,1}$ and $\xi_{1,2}=\xi_{0,1}=1$. The filled and empty circles are experimental data for $\mathrm{NO}(\mathrm{v}=0 \rightarrow 1)$ and $\mathrm{NO}(\mathrm{v}=0 \rightarrow 2)$, respectively. The dashed and lines are the results of the kinetic model for $\mathrm{NO}(\mathrm{v}=0 \rightarrow 1)$ and $\mathrm{NO}(\mathrm{v}=0 \rightarrow 2)$. The dot-dashed lines are the thermal limit. The incidence energy was $0.93 \mathrm{eV}$.

It is possible that one may estimate, in units of seconds, $\tau_{\text {eff. }}$ We can estimate a critical distance for vibrationally promoted electron emission ${ }^{9}, \mathrm{Z}_{\mathrm{C}} \sim 5 \AA$, based on a previous paper, despite 
several important differences. From the measured velocity of the NO molecular beam, vNo, we estimate the round-trip time of the collision, $\tau_{\text {eff }}$, within a constant velocity approximation.

$$
\tau_{\text {eff }}=\frac{2 * Z_{C}}{v_{\mathrm{NO}}} \approx 400 f s
$$

This analysis implies that electron mediated energy transfer provides a path to molecule-surface equilibrium on the sub-ps time scale, consistent with vibrational lifetime measurements for small molecules on metal surfaces ${ }^{101}$. This is compared with vibrational relaxation coupled to phonons, which can be several orders of magnitude slower. ${ }^{71,102,103}$

In characterizing the excitation of $\mathrm{NO}(\mathrm{v}=0 \rightarrow 2)$, it is important to distinguish between direct and sequential excitation. Since production of $\mathrm{NO}(\mathrm{v}=0 \rightarrow 1)$ is always at least $10 x$ more efficient than production of $\mathrm{NO}(\mathrm{v}=2)$, under the experimental conditions used here, it is reasonable to think that $\mathrm{NO}(\mathrm{v}=2)$ might be produced by two single-quantum excitation steps $\mathrm{NO}(\mathrm{v}=0 \rightarrow 1 \rightarrow 2)$ and not by a vibrational overtone mechanism.

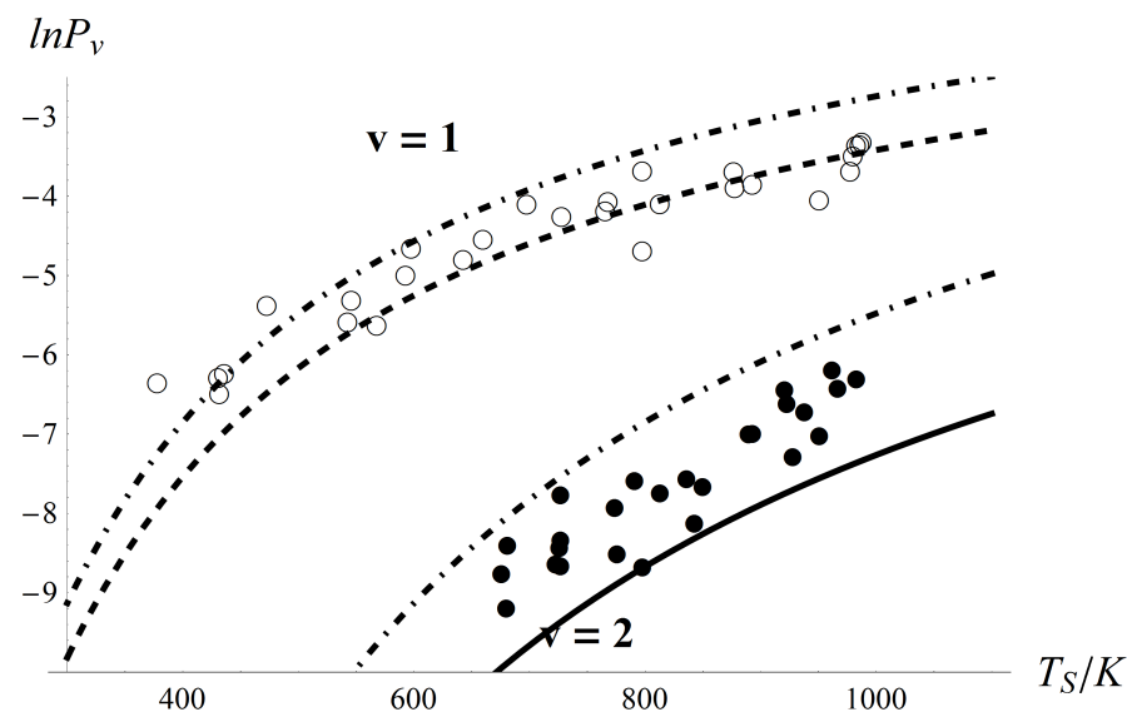

Figure 6.3 The importance of vibrational overtone excitation. This is the resulting fit if $\xi_{0,2}=0$, and otherwise, the fitting parameters are unchanged from the fit used in Fig. 6.2.

Figure 6.3 shows the impact on the fit if vibrational overtone pumping is neglected, that is setting $\xi_{0,2}=0$. While this change has no noticeable effect on the predicted $\mathrm{v}=1$ populations, the $\mathrm{v}=2$ excitation probability is underestimated. To obtain a reasonable fit when overtone pumping is neglected, $\xi_{1,2}$ has to become unreasonably large, $\xi_{1,2}>12 * \xi_{0,1}$. There is no reason to think that $\xi_{1,2}$ should be much larger than $\xi_{0,1}$, as indicated by the full fit. In contrast, one may start with the best fit parameters of Fig. 5 and change $\xi_{1,2}$ to 0 , with no detectable effect to the fit.

A simple picture which may help to show that direct excitation is important is that if $\mathrm{NO}(\mathrm{v}=1 \rightarrow 2)$ occurred with the same magnitude of $(\mathrm{v}=0 \rightarrow 1)$ then at $\mathrm{T}_{\mathrm{s}}=1000 \mathrm{~K}$ where the 
$\mathrm{NO}(\mathrm{v}=0 \rightarrow 1)$ excitation probability is $\sim 3.5 \%$, the excitation probability for $\mathrm{NO}(\mathrm{v}=2)$ would be $0.12 \%$. This is less than the experimentally determined excitation probability for $\mathrm{NO}(\mathrm{v}=0 \rightarrow 2)$ is $\sim 0.20 \%$. Therefore, we conclude that $\mathrm{v}=2$ is predominantly populated by direct vibrational overtone excitation. Indeed, the best fit results, $\xi_{0,2}=0.30 * \xi_{0,1}$, suggest that the multi-quantum vibrational excitation is almost as strong as single quantum excitation. At other incidence energies the ratio of $\xi_{0,2}$ to $\xi_{0,1}$ is much closer to 1 . This can qualitatively be seen in Fig. 6.2. It is likely that within the spread of data of this experiment, $\alpha_{02}$ and $\alpha_{01}$ do not vary significantly from each other. This is strong evidence that vibrational excitation occurs predominantly via direct $\mathrm{NO}(\mathrm{v}=0 \rightarrow 2)$ overtone excitation. This contrasts starkly with optical overtone transition probabilities. For example for NO, the $1^{\text {st }}$ optical overtone strength is 0.015 that of the fundamental. ${ }^{57}$

\subsection{Analytical kinetic model:}

In the previous kinetic model the integrals were solved numerically. Additional insight can be drawn by integrating analytically. ${ }^{96}$ While there are several additional points of information that can be derived from the analytical model, only the limiting values to the Arrhenius pre-exponent will be discussed in detail. The kinetic model showed that the Arrhenius form was a low temperature (compared to vibrational energy transfer) form of a more general solution. At surface temperatures below $1100 \mathrm{~K}$ no deviation between the kinetic model and the Arrhenius function is expected.

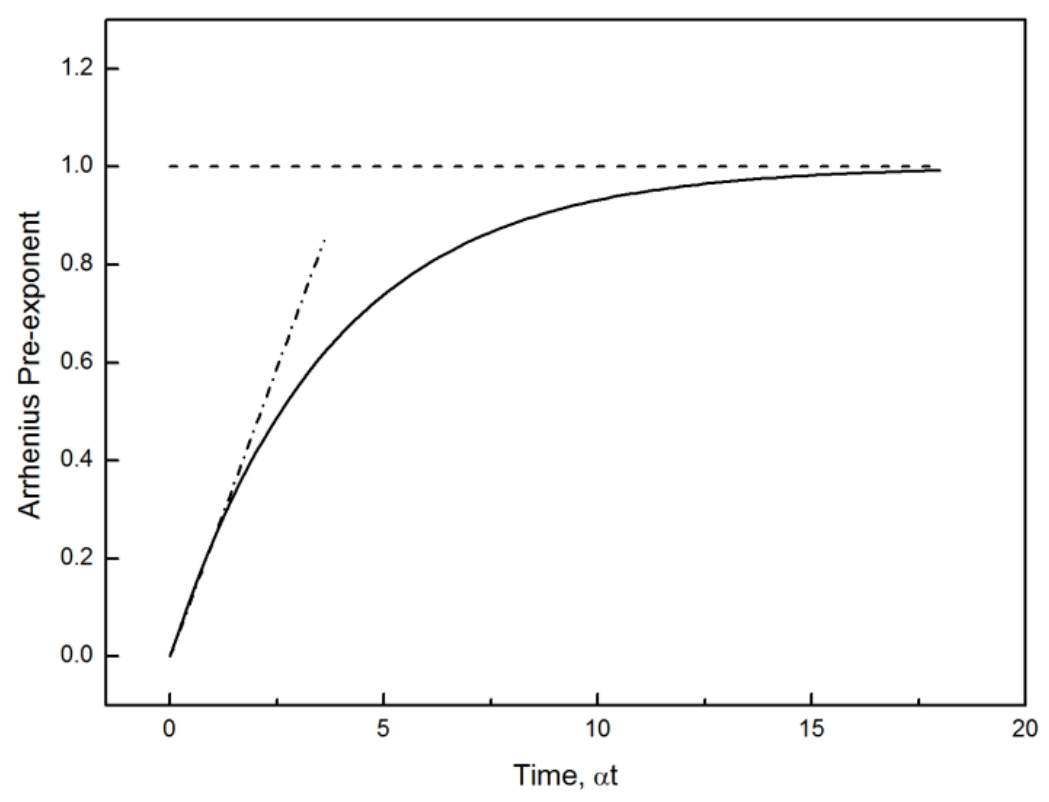

Figure 6.4 Dependence on the interaction time of the low temperature limit Arrhenius pre-exponent of the vibrational excitation probability of the single-step mechanism, solid line. At short interaction times the linear growth, dot dashed line, from zero, followed by saturation to unity, dashed line. 
The pre-exponent of this Arrhenius form is almost independent of the temperature of the surface, and its dependence on the interaction time is shown in Fig. 6.4. The pre-exponent starts off linearly at short times and approaches a limiting value of unity given by as the interaction time goes to infinity. Unity, the upper bound of the Arrhenius pre-factor, describes complete thermal equilibrium with the surface.

One of the most important extensions of the kinetic model is that it allows physical interpretation of the Arrhenius pre-exponential factor, which has long been an empirical parameter in the description of electronically non-adiabatic vibrational energy transfer. Specifically, it is now clear, that the previously seen Arrhenius behavior is an approximation, in the limit of low surface temperatures relative to the vibrational spacing, of a more general solution. Although the exponential term with the activation energy equal to the difference in energy between the vibrational states has previously been suggested to arise from the thermal distribution of electron-hole pairs in the metal, ${ }^{8}$ the nature of the pre-exponential factor remained largely unexplained. Kinetic analysis of the elementary excitation processes combined with analytical solutions to the electron statistics shows explicitly that the Arrhenius pre-exponential factor may only assume values between zero and unity.

The kinetic model approach has the potential to become a general procedure for the analysis of experimental data on electronically non-adiabatic vibrational energy transfer. It especially may be useful for the comparison between systems. Especially attractive is the possibility to use the Arrhenius pre-factors as a comparison between different systems.

It is clear that the general validity of this model has to be tested further on additional moleculesurface systems. The electronically non-adiabatic vibrational excitation data available for NO on two other metals, $\operatorname{Ag}(111)^{8}$ and $\mathrm{Cu}(110)^{11}$, is already controversial, as the Arrhenius pre-factors are larger than unity: 2.0 for $\mathrm{NO} / \mathrm{Ag}$ and 1.3 for $\mathrm{NO} / \mathrm{Cu}$. According to the results of the kinetic analysis above this would not be possible, as it implies the observed populations of vibrationally excited product were larger than the limit of thermal equilibrium with the surface. However absolute excitation probabilities are remarkably difficult to measure and this discrepancy could arise from experimental inaccuracies. Furthermore for both cases the experimental error bars would bring the data into agreement with the thermal limit. Alternatively it might be that a certain fraction of the vibrational excitation results from a translational to vibrational mechanism. More experiments are surely needed to resolve this disagreement and to possibly refine the proposed model.

One interesting comparison is to vibrational excitation of $\mathrm{HCl}$ scattering from $\mathrm{Au}(111)$. All $\mathrm{NO} /$ metal systems show substantially higher excitation probabilities than those of $\mathrm{HCl}, \sim 2-4$ orders of magnitude. Even the multi-quantum $\mathrm{NO}(\mathrm{v}=0 \rightarrow 2)$ excitation probabilities, which has a larger activation energy of $0.472 \mathrm{eV}$, are higher than these of $\mathrm{HCl}(\mathrm{v}=0 \rightarrow 1)$, which has an activation energy of $0.371 \mathrm{eV}$. It is clear, that the inherent propensity for vibrational excitation is significantly higher for $\mathrm{NO}$ then for $\mathrm{HCl}$. However, comparison of the pre-factors gives a more 
quantitative comparison. For vibrational excitation of $\mathrm{HCl}(\mathrm{v}=0 \rightarrow 1)$ scattering from $\mathrm{Au}(111)$ at $\mathrm{E}_{\mathrm{i}}=1.12 \mathrm{eV}$ the Arrhenius pre-factor is $1.9 \times 10^{-3}$, compared to the pre-factor of $\sim 0.7$ seen in vibrational excitation of $\mathrm{NO}(\mathrm{v}=0 \rightarrow 1)$ scattering from $\mathrm{Au}(111)$ and this indicates that $\mathrm{NO}(\mathrm{v}=0 \rightarrow 1)$ has a coupling, independent of the surface electron statistics of $\sim 400 x$ more than that of $\mathrm{HCl}(\mathrm{v}=0 \rightarrow 1)$.

In this section I have shown that a kinetic model can allow for deeper insight to be drawn from the surface temperature dependence of vibrational excitation. I have shown that under the experimental conditions used for the measurement of vibrational excitation probabilities of $\mathrm{NO}(\mathrm{v}=0 \rightarrow 1,2)$ scattering from $\mathrm{Au}(111)$ the Arrhenius approximation does not noticeably deviate from the full kinetic model I have also given meaning to the Arrhenius pre-factor, which until now had been widely ignored. 


\section{Origin of $E_{i}$ Dependence:}

Typically the incidence translational energy dependence for nonadiabatic vibrational excitation is rationalized by the fact that the molecule can penetrate deeper into the electron cloud of the metal surface, enhancing the coupling at higher incidence translational energies. In this section, a more quantitative picture will hopefully shed some light on this phenomenon. I will use NO vibrational excitation, measured as a function of incidence translational energy results of which are shown in section 5, to build this model for the incidence energy of translation dependence for nonadiabatic vibrational excitation. I will then draw some generalizations from these results and compare them to vibrational relaxation of $\mathrm{NO}(\mathrm{v}=18)$ scattering from $\mathrm{Cs}$ decorated $\mathrm{Au}(111)$ where an inverse incidence energy of translation dependence of vibrational energy change is seen. $^{9}$

To explain the incidence translational energy dependence, I will combine the kinetic model seen in section $6^{95,96}$ with the formulation of Newns ${ }^{31}$ in which the rate constants for vibrational excitation of $\mathrm{NO}$ when scattered from $\mathrm{Au}(111)$, vary as a function of molecule surface distance, and consequently time. These rate constants are determined by the overlap with the distance dependent density of states (PDOS), and the time and distance are linked via the velocity of the molecule, which can be obtained by the initial kinetic energy.

The molecule surface interaction potential, from Newns, is modeled in Morse shape by the equation:

$$
U(z)=D_{0}\left(e^{-2 \alpha z}-2 e^{-\alpha z}\right)
$$

Here $D_{0}$ is the depth of the well (binding energy) and $\alpha$ is the parameter defining the "width" of the well, this $\alpha$ is not the same as the $\alpha$ representing coupling strength in the kinetic model. I define the zero of the molecule surface distance axis, $\mathrm{z}$, as the minimum of this potential. It is shown in Fig. 7.1 with some very reasonable parameters, $D_{0}=300 \mathrm{mEv}$ and $\alpha=1.1 \AA^{-1}$. Newns has some justification for both and uses 300 and 0.95 for $D_{0}$ and $\alpha$, respectively. 


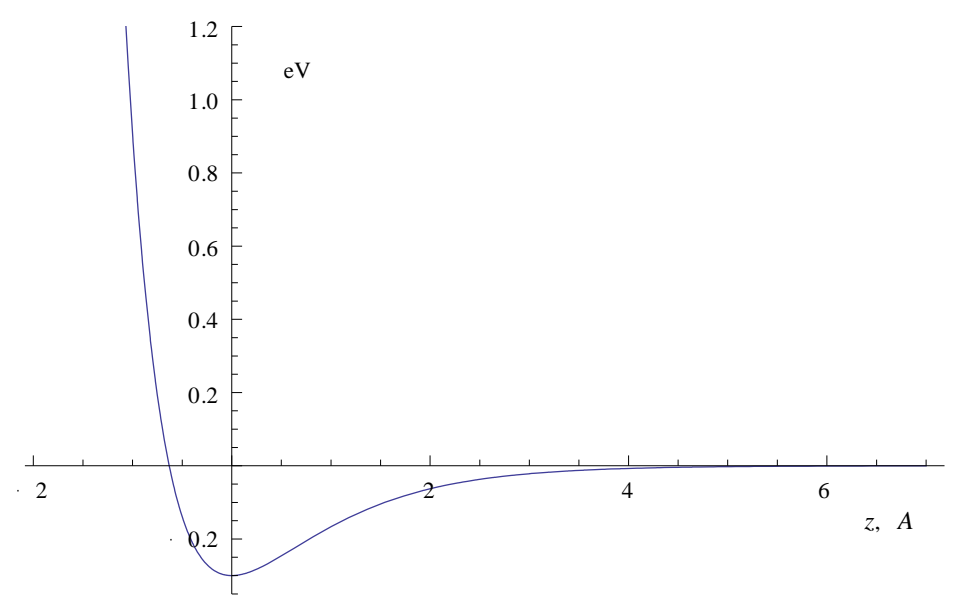

Figure 7.1 Interaction potential of the molecule and surface plotted as a function of the molecule surface distance, $\mathrm{z}$, as given by Eq. (7.1), with the values mentioned in the text.

For a given incidence translational energy and the potential shown in Fig. 7.1, we can calculate the velocity of the molecule as a function of the molecule surface distance, z. An example of such a trajectory is shown in Fig. 7.2, with an $\mathrm{E}_{\mathrm{i}}=0.93 \mathrm{eV}$.

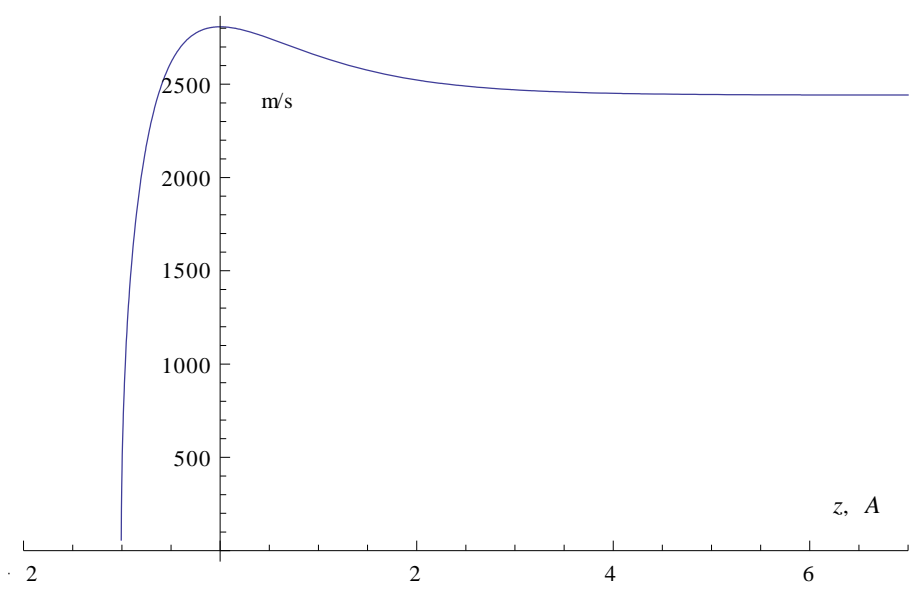

Figure 7.2 Velocity of a molecule, as a function of $\mathrm{z}$, as it approaches the surface over the potential $U(z)$.

The turning point of the molecular trajectory, as can be seen in Fig. 7.2 when the velocity reaches zero, occurs on the repulsive wall about $1 \AA$ past the minimum of the potential $U(z)$. After reaching the turning point, the molecule leaves the surface over the same trajectory, eventually acquiring the initial velocity and consequently the initial kinetic energy. Here it is clear that any translational energy transfer mechanism is not included in this model.

Another important parameter of the model is the stabilization of the affinity level by the image charge interaction, which is given by: 


$$
E(z)=E_{\infty}-\frac{e^{2}}{4\left(z-z_{0}\right)}, \quad E_{\infty}=\Phi-E_{a}
$$

$\Phi$ and $E_{a}$ represent the work function of the surface and adiabatic electron affinity of the molecule, respectively, with $z_{0}$ being the position of the image plane of the electrostatic image charge interaction. In both the above equation and through the rest of the model zero of energy is set at the Femi level of the metal. To obtain actually correct numerical values, an extra coefficient is needed in the Columbic term in Eq. (7.2), which depends on the chosen units. The affinity level given by Eq. (7.2) is plotted in Fig. 7.3 for $\Phi=4.8 \mathrm{eV}$ and $E_{a}=0.02 \mathrm{eV}$ and $z_{0}=-1.83$ $\AA$, which is near the value used by Newns $\left(z_{0}=-1.93 \AA\right)$.

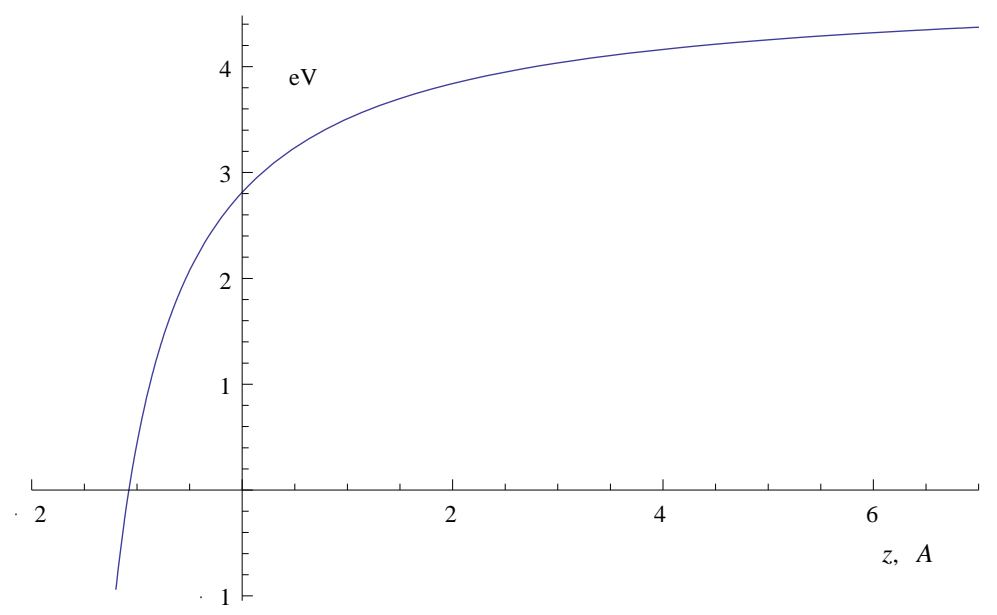

Figure 7.3 Molecular affinity level stabilized by the image charge interaction plotted as a function of $\mathrm{z}$.

Next, the dependence of the broadening of the affinity level on the molecule-surface distance must be understood. It appears that an exponential decay away from the surface had been suggested by a number of authors ${ }^{8,29,31}$ given by:

$$
\Delta(z)=\Delta_{0} e^{-2 \alpha z}
$$

with two parameters, $\Delta_{0}$ and $\alpha$. Newns argues that $\alpha$ in Eq. (7.3) can be taken equal to that in the Morse potential Eq. (7.1), which indeed worked for him and is utilized here. Both parameters are adjusted by hand to obtain the best fit through the experimental data, and with the best approximate fit of $\Delta_{0}=0.11 \mathrm{eV}$ and $\alpha=1.1 \AA^{-1}$ the affinity level broadening is plotted in Fig. 7.4. 


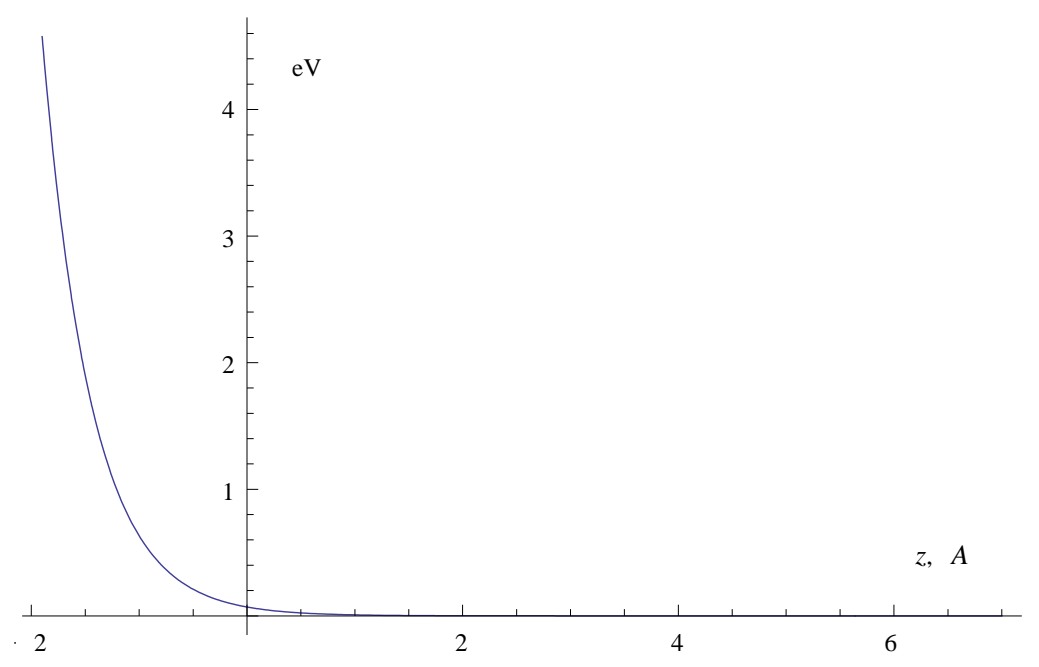

Figure 7.4 Affinity level broadening as a function of the molecule surface distance.

Finally, having the expressions for the affinity level Eq. (7.2) and it's broadening Eq. (7.3) the PDOS can be calculated using a formula utilized by Newns and others: ${ }^{104}$

$$
\rho(z)=\frac{1}{\pi} \frac{\Delta(z)}{\Delta(z)^{2}+E(z)^{2}}
$$

The results from Eq. (7.4) are plotted in Fig. 7.5. The most important aspects of this model are the molecular trajectory, which depends on the initial velocity, and the PDOS, as the interplay between them will govern the overall incidence translational energy dependence. Here the PDOS determines the interaction region in which significant vibrational energy transfer rates occur, and the former defines the time molecules spend in that region.

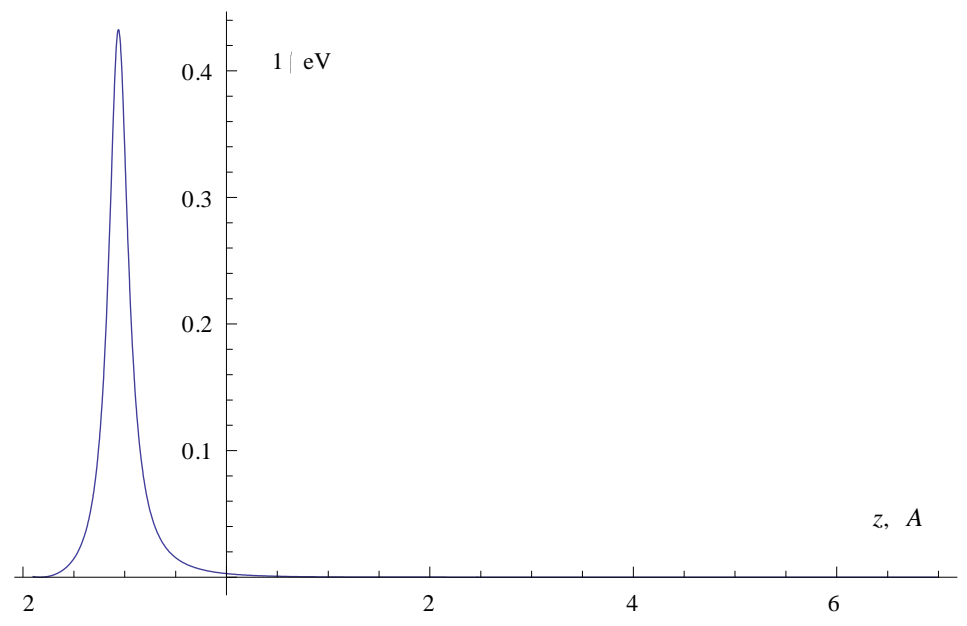

Figure 7.5 PDOS as a function of $z$. 
Figure 7.2 shows that the molecules at $\mathrm{E}_{\mathrm{i}}=0.93 \mathrm{eV}$ turns around at $-1 \AA$ on the $\mathrm{z}$ axis, which corresponds roughly to the middle of the PDOS, shown in Fig. 7.5. As the incidence energy of translation grows the regions of higher PDOS will be accessed by the molecules, increasing the vibrational excitation probability. As PDOS has significant values in a narrow region outside the surface, it would be expected that at some high incidence energy of translation the incoming molecules would sample the entire PDOS region, but would pass through the region so fast as to not allow much time for energy transfer. The balance between sampling of the region and the time spent in the region will give rise to a maximum vibrational energy excitation at a specific incidence translational energy.

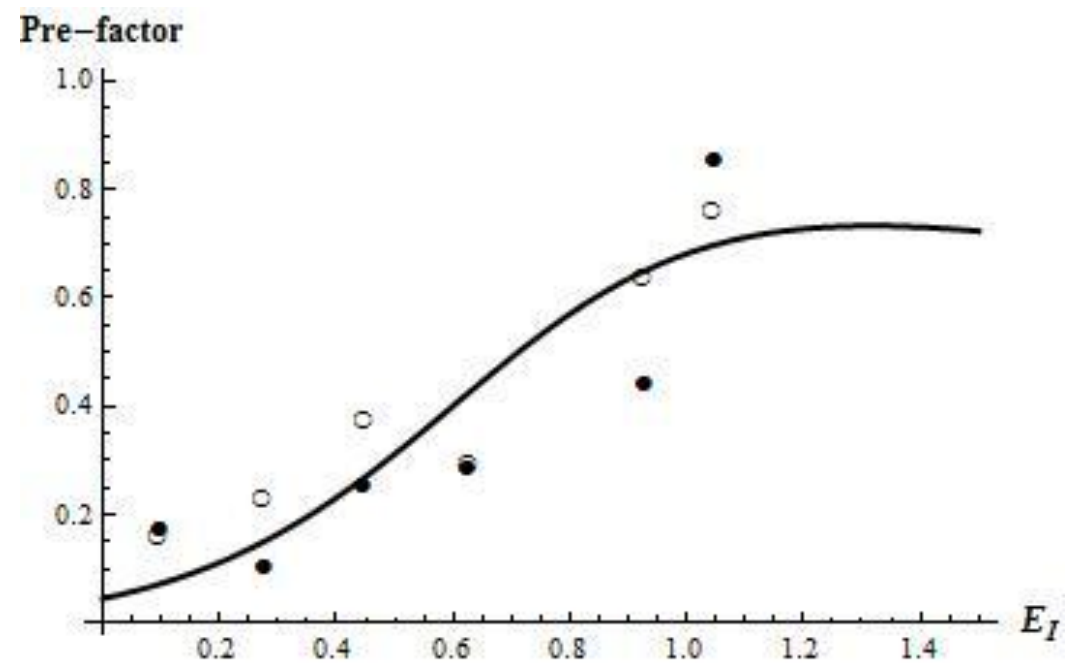

Figure 7.6 Arrhenius pre-factor as a function of incidence energy of translation. The filled circles represent the temperature independent pre-factor for $\mathrm{NO}(\mathrm{v}=0 \rightarrow 2)$ excitation. Empty circles represent the temperature independent pre-factor $\mathrm{NO}(\mathrm{v}=0 \rightarrow 1)$ excitation. The solid line represents the fit from the model to the experimental data.

From the considerations laid out in this section, it can be seen that the interplay between the molecule velocity and the PDOS gives rise to the incidence translational energy dependence for vibrational excitation of $\mathrm{NO} / \mathrm{Au}(111)$. Similar arguments can be made for vibrational excitation of NO scattered from $\mathrm{Ag}(111)$ and $\mathrm{Cu}(110)$ as well as $\mathrm{HCl}$ scattered from $\mathrm{Au}(111) .{ }^{8,11,12}$

Another work of vibrational energy change measured as a function of incidence energy of translation was that of $\mathrm{NO}(\mathrm{v}=18)$ relaxation when scattered from a Cs decorated $\mathrm{Au}(111)$ surface. In this work electron emission, which corresponds to a large amplitude vibrational relaxation, from the surface was measured as a function of incidence translational energy. Electron emission decreased with increasing incidence translational energy. ${ }^{9}$ 


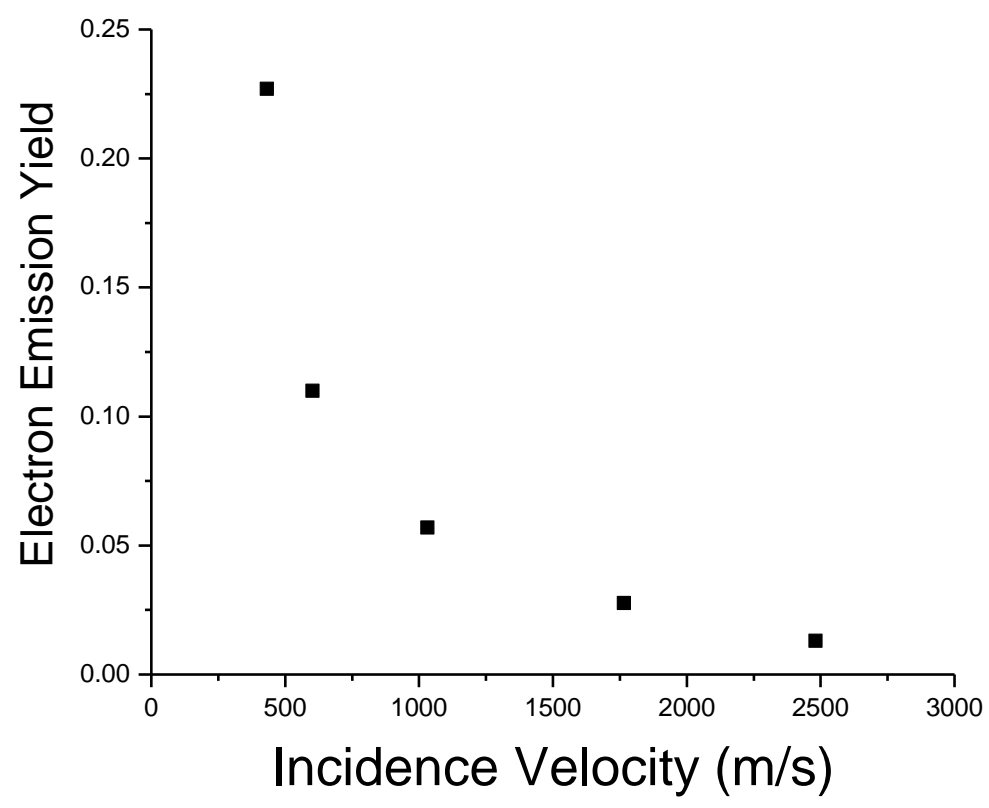

Figure 7.7 Electron emission yield from a Cs decorated $\mathrm{Au}(111)$ surface as a function of incidence velocity of the $\mathrm{NO}(\mathrm{v}=18)$ molecules with $\mathrm{T}_{\mathrm{s}}=300 \mathrm{~K} .^{9}$

At first glance it appears that the results of $\mathrm{NO}(\mathrm{v}=18)$ relaxation, shown in Fig. 7.7, do not agree with the trajectory based model developed to describe vibrational excitation, however this is not the case. One very important difference between the $\mathrm{NO}(\mathrm{v}=0 \rightarrow 1,2)$ vibrational excitation, the $\mathrm{NO}(\mathrm{v}=18)$ relaxation, is the surface from which the molecules scatter. As mentioned earlier the incidence translational energy dependence arises from the interplay between the PDOS and the velocity of the molecule. When the work function of the surface is lowered, this has a strong effect on the PDOS, both in magnitude and their distance from the surface. Although the surface of $\mathrm{Cs}$ decorated $\mathrm{Au}(111)$ must vary in other ways besides work function, the NO Cs/Au(111) interaction potential was approximated as the same as that of $\mathrm{NO} / \mathrm{Au}(111)$ described above, the only alteration was an adjustment of the work function from $\mathrm{Au}(111)$ to that of $\mathrm{Cs} / \mathrm{Au}(111)$ previously measured. ${ }^{44}$ This adjustment made the PDOS much stronger and shifted it to the more positive direction on the $\mathrm{z}$ axis. This allows molecules with any incidence energy of translation to interact with the most intense aspects of the PDOS. Here the velocity determines the time the molecules spend in the PDOS and consequently gives rise to an inverse velocity dependence.

In explaining these results the authors gave a very similar argument to the one used here, where an interaction region far from the surface is responsible for the energy exchange. The time in the interaction region is determined by the velocity, and the faster the velocity the lower the time. This trajectory based allows for a unification of the incidence translational energy dependences for vibrational excitation of $\mathrm{NO}(\mathrm{v}=0 \rightarrow 1,2)$ scattering from $\mathrm{Au}(111)$ and vibrational relaxation of $\mathrm{NO}(\mathrm{v}=18)$ scattering from a Cs decorated $\mathrm{Au}(111)$ surface. 


\section{Conclusions and Outlook:}

To summarize I studied energy transfer between gas phase molecules and a metal, $\mathrm{Au}(111)$ surface. I studied translational energy transfer for both $\mathrm{NO}$ and $\mathrm{HCl}$. There was no dependence on the incident vibrational state for the final translational energy distributions in vibrationally elastic scattering. However, molecules that underwent vibrational relaxation upon scattering had more energetic translational energy distributions. When the molecule, $\mathrm{NO}$ or $\mathrm{HCl}$, vibrationally relaxed a portion of the lost vibrational energy was coupled to translation of the molecule. Possible mechanisms for this coupling were discussed; with the most likely being that intrinsic coupling between vibration and translation exists.

In vibrationally elastic scattering, for both $\mathrm{HCl}$ and $\mathrm{NO}$, a sizeable fraction of the scattered molecules have translational energies below that of the Baule limit, the limit of kinetic energy transfer in a mechanical picture. This is more translational energy transfer than has been previously measured in molecule or atomic interactions with surfaces, at thermal energies.

Vibrational excitation probabilities of $\mathrm{NO}(\mathrm{v}=0 \rightarrow 1,2)$ scattered from $\mathrm{Au}(111)$ were measured as a function of surface temperature and incidence energy of translation. The vibrational excitation followed the well-known Arrhenius surface temperature dependence typically seen for electronically non-adiabatic vibrational excitation. This is true for both single and multi-quanta vibrational excitation, with an Arrhenius activation energy equal to the vibrational energy spacing.

I developed a kinetic model to gain deeper insight into the dynamics of the vibrational excitation mechanism. It was seen that excitation of $\mathrm{NO}(\mathrm{v}=0 \rightarrow 2)$ occurs predominantly via direct excitation rather than through sequential single-quanta excitations. The kinetic model also gave meaning to the Arrhenius pre-factor, which previously had been largely ignored. Notably, it showed that the pre-factor can range between zero and unity and that its value approximates the strength of the coupling between the vibration in the molecule and electron hole pairs in the metal.

In the near future an extensive comparison to first principles theory, independent electron surface hopping, seen in section 2.5 , for NO vibrational excitation is currently underway. A preliminary comparison shows good agreement between theory and experiment for vibrational excitation probabilities. IESH also computes angular, rotational, and translation distributions of the final molecules, all of which can be compared to experimental results. This large set of experimental data, presented in this thesis, can hopefully help refine theoretical models of nonadiabatic energy transfer and help it be extended to other molecule surface systems.

The possibilities for future work on this machine, with the aim of elucidating the dynamics of molecule surface interactions, are limited only by time. One of the first possibilities is to look at vibrational excitation to higher vibrational states, such as $\mathrm{NO}(\mathrm{v}=0 \rightarrow 3)$. Two color REMPI is 
more sensitive that one color, and can be employed to detect less likely events, such as vibrational excitation to levels higher than $\mathrm{NO}(\mathrm{v}=2)$. A simple extension of the kinetic model can be made for higher vibrational states, and the coupling strength of the direct $\mathrm{NO}(\mathrm{v}=0 \rightarrow 3)$ transitions can be derived.

Another study of some interest would be the measurement of the final translational energy of vibrationally excited molecules. This would be analogous to the translational measurements of vibrational relaxation measured in this thesis. These measurements would however help elucidate the mechanism of $\mathrm{T}-\mathrm{V}$ coupling, for example if the translational energy is higher with vibrational excitation, then acceleration due to the induced image charge is then the likely pathway for the observed T-V coupling. However, if the translational energy decreases with vibrational excitation than the direct coupling between the vibration and translation of the molecule is the likely mechanism for the T-V coupling.

Experiments of translational and vibrational energy transfer for other molecule surface systems would also clearly be of interest. Perhaps, especially of interest, would be scattering experiments from surfaces covered in nano-particles. This would be quite relevant as most catalytic systems occur on ill-defined surfaces, which may be better approximated by surfaces covered in nanoparticles than by the single crystals typically used in current molecular beam scattering experiments. 


\section{Appendices:}

\section{Appendix I: Conversion of TOF data to translational energy distributions:}

This appendix deals with the transformation of measured TOF distributions to translational energy distributions.

Our TOF measurements use REMPI to detect the molecules. REMPI is a density sensitive technique, that is, the ion signal is proportional to the number density of molecules in the quantum state being probed. The TOF Signal, $S(t)$ is measured as a function of time delay between the firing of the laser for vibrational overtone pumping and the laser used for REMPI detection. We convert this TOF density to flux by multiplying by the velocity, $v(t)$.

$$
v(t)=l / t
$$

where 1 is the distance between the pump and REMPI lasers beams. This results in a flux of

$$
I(t) d t=S(t) v(t) d t
$$

To transform from the time domain to velocity space, we must conserve probability in each differential time (velocity) element:

$$
P(v) d v=I(t) d t=S(t) v(t) d t
$$

Some simple math gives:

$$
P(v)\left|\frac{d v}{d t}\right|=S(t) v
$$

The Jacobian is given by:

$$
\left|\frac{d v}{d t}\right|=\frac{\ell}{t^{2}}=\frac{v^{2}}{\ell}
$$

Applying Eq. (A1.5) to Eq. (A1.4) we arrive at the proper transformation:

$$
P(v)=S(t) \frac{l}{v}
$$

This equation is used to convert raw molecular beam TOF data to velocity space $P(v)$. We fit the data converted to velocity space using a velocity distribution function ${ }^{83}$ of the form:

$$
P(v)=A v^{3} e^{-\left(\frac{v-v_{0}}{\alpha}\right)^{2}}
$$


The best fit to the above function as well as the data can be converted to energy space, $P(E)$. This is done in a similar way as just described. Here:

$$
P(E) d E=P(v) d v
$$

and the proper Jacobian has the form:

$$
\left|\frac{d E}{d v}\right|=m v=\sqrt{2 m E}
$$

Combining Eq. (A1.8) and Eq. (A1.9) with Eq. (A1.5) results in:

$$
P(E)=\frac{P(v)}{m v}=\frac{S(t) l}{m v^{2}}
$$

For additional information here is the fitting function Eq. (A1.7) in time:

$$
P(t)=A\left(\frac{l}{t}\right)^{4} e^{-\left(\frac{l}{\alpha}\right)^{2}\left(\frac{1}{t}-\frac{1}{t_{0}}\right)^{2}}
$$

and energy space:

$$
P(E)=A \frac{E}{m^{2}} e^{-\frac{2}{m \alpha^{2}}\left(\sqrt{E}-\sqrt{E_{0}}\right)^{2}}
$$

where $\mathrm{t}_{0}=v_{0} l l$ and $E_{0}=1 / 2 \mathrm{~m} v_{0}^{2}$. 


\section{Appendix II Additional measurements for ion collection efficiency:}

This appendix shows measurements of ion collection efficiency taken at various positions of the REMPI laser. These measurements clarify the importance of the location of the REMPI laser for both translational energy and vibrational excitation measurements.

Below, the figures show ion collection efficiency, of background $\mathrm{HCl}$ leaked into the chamber, along the molecular beam axis, as a function of distance from the surface. The figure on the left is with the surface in the appropriate location for scattering, the right with the surface raised far above the molecular beam. Note the difficulty in collecting ions without the surface. For all cases below the lower ion optic was $1150 \mathrm{~V}$ and the upper $1500 \mathrm{~V}$.
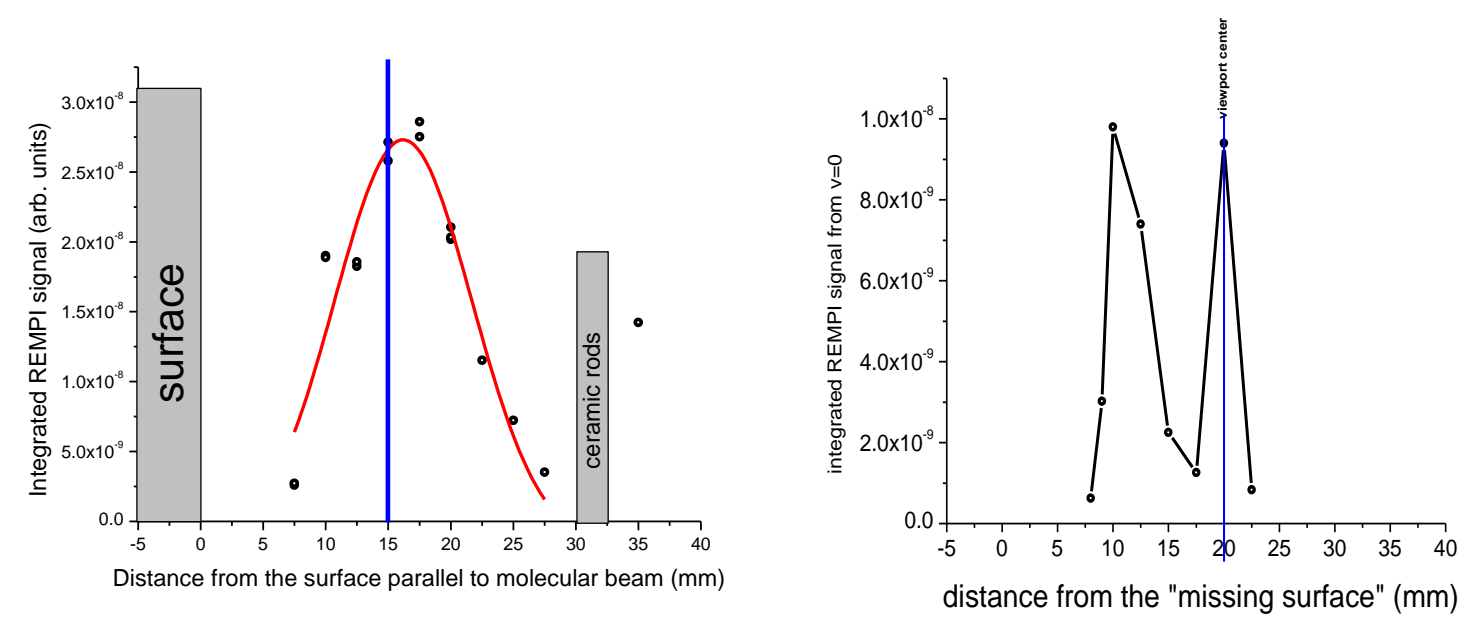

All the measurements below correspond to when the surface is in the location for scattering experiments. They take place on background $\mathrm{HCl}$ leaked into the chamber. The absolute collection efficiency is not the most important feature here; the main point is consistency for ion collection as a function of angle to the incident beam. The less this varies the easier and more reliable the data analysis for angular distributions becomes. 

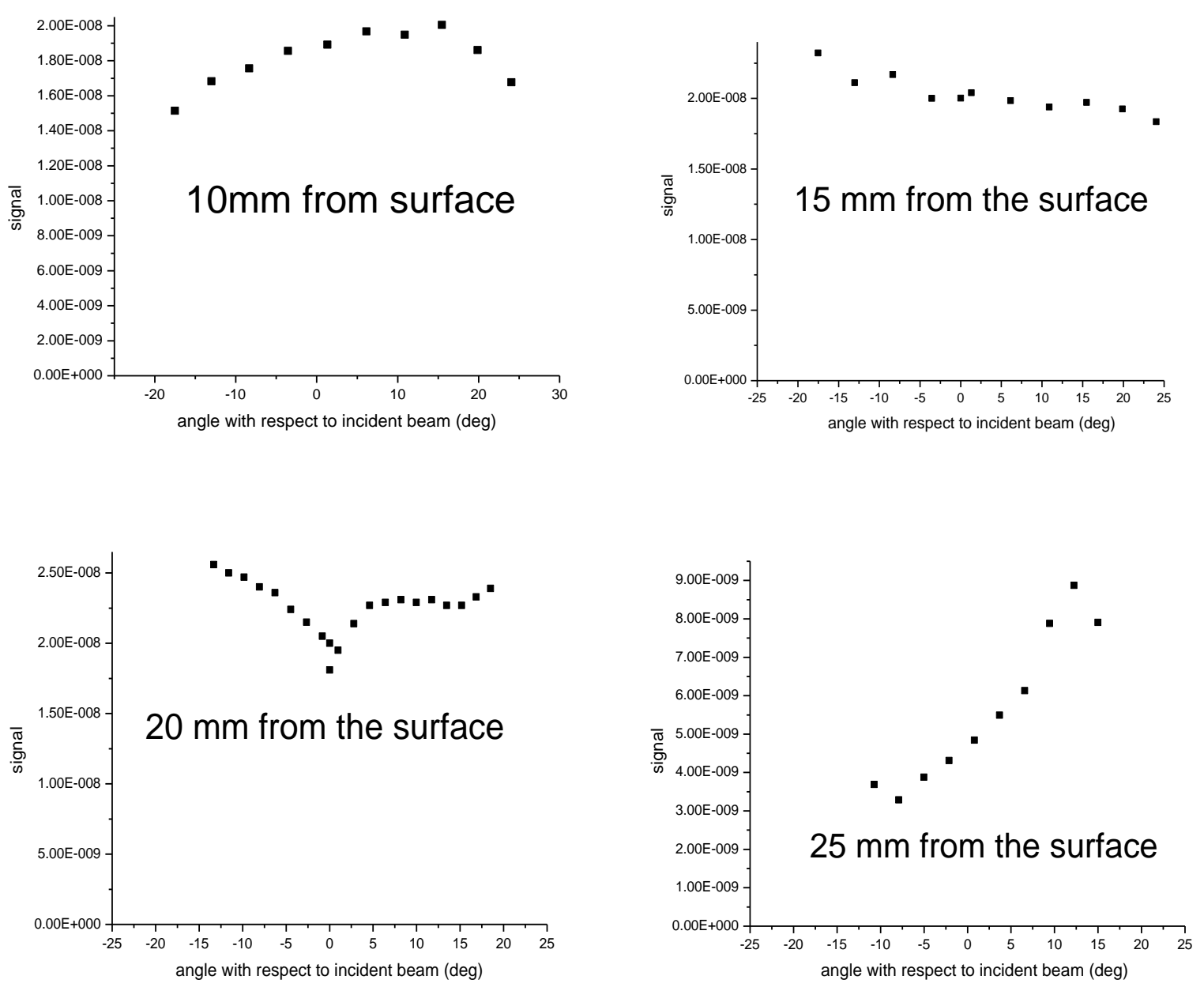


\section{Appendix III: Determination of surface angle:}

This appendix deals with the determination of the surface angle relative to the molecular beam. This is important as small differences in this angle can induce systematic error into the measurement of the distance between the surface and the pump laser. Proper analysis of translational energy transfer to the surface, and to a lesser extent vibrational excitation probabilities, requires an accurate knowledge of this angle and the surface laser distance.

The distance between the pump laser and the surface is measured by moving the surface with a micro-metric translational stage until the surface eclipses the pump laser beam. If the surface is angled slightly the edge of the surface eclipses the pump laser beam first. As the molecular beam strikes the center of the crystal, this leads to an incorrect distance between the surface (where the molecular beam strikes) and the pump laser beam. However, I developed a way to measure this angle. I tightly focused the IR laser and moved the surface forward until the beam was halfway eclipsed. By adjusting the rotation of the crystal on the manipulator and again moving the crystal forward I was able to look for the rotational position which gives the maximum distance between the surface and the pump laser beam. This is shown in Fig. A3.1.

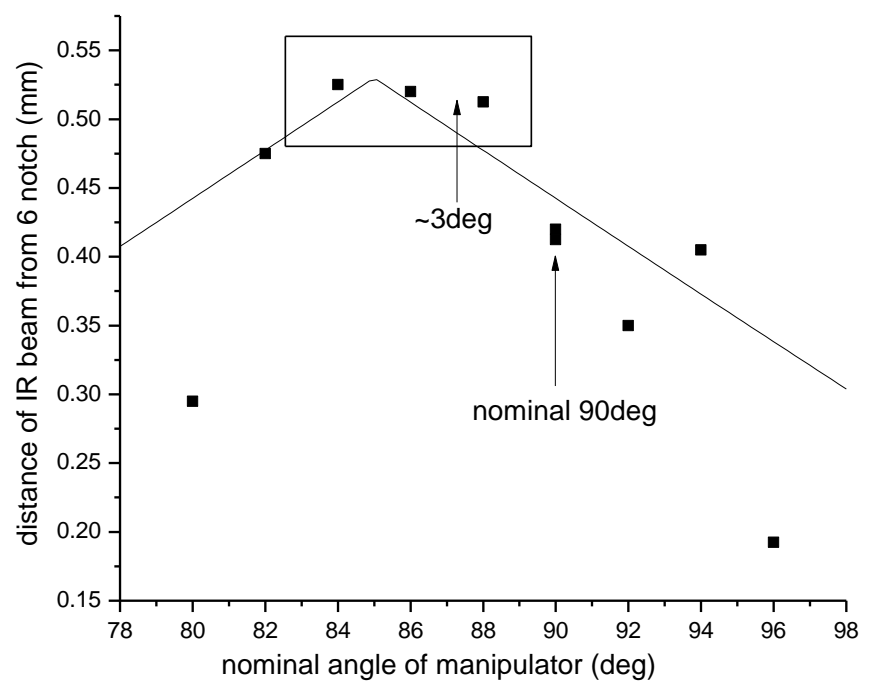

Figure A3.1 It is clear that nominal $90^{\circ}$ is not true $90^{\circ}$. Distance from the surface to the IR beam, located at the $6^{\text {th }}$ notch on the alignment tool, measured as a function of nominal manipulator angle.

Figure A3.1 shows that nominal $90^{\circ}$ taken from the manipulator does not correspond to true $90^{\circ}$. There appears to be around a $3^{\circ}$ offset. Using this offset and the manipulator geometry I 
calculated an additional distance from the IR laser to the surface of $0.24 \mathrm{~mm}$. Table A3.1 shows the how altering the distance between the IR laser and the surface affects the analysis of the data.

Table A3.1 Change in the scattered translational energy of the $\mathrm{HCl}(\mathrm{v}=2 \rightarrow 2)$ channel by the correction to the flight distance. The appropriate correction is $0.24 \mathrm{~mm}$.

\begin{tabular}{|l|l|l|}
\hline IR-surface distance & $\left\langle\mathrm{E}_{22}\right\rangle$ & $\begin{array}{l}\text { Fraction of translational energy } \\
\text { retained, } \mathrm{E}_{\mathrm{i}}=.275 \mathrm{eV}\end{array}$ \\
\hline Originally measured: $2.951 \mathrm{~mm}$ & $0.107 \mathrm{eV}$ & 0.389 \\
\hline $\begin{array}{l}\text { Adding } 0.1 \mathrm{~mm} \text {, i.e } 3.051 \mathrm{~mm} \\
\text { distance }\end{array}$ & $0.110 \mathrm{eV}$ & 0.400 \\
\hline $\begin{array}{l}\text { Adding } 0.2 \mathrm{~mm} \text {, i.e } 3.151 \mathrm{~mm} \\
\text { distance }\end{array}$ & $0.112 \mathrm{eV}$ & 0.407 \\
\hline $\begin{array}{l}\text { Adding } 0.24 \mathrm{~mm} \text {, i.e } 3.191 \mathrm{~mm} \\
\text { distance }\end{array}$ & $0.113 \mathrm{eV}$ & 0.411 \\
\hline
\end{tabular}

It should be noted that after correction the translational energy distributions for the $\mathrm{HCl}(\mathrm{v}=2 \rightarrow 2)$ and $\mathrm{HCl}(\mathrm{v}=0 \rightarrow 0)$ channels are not distinguishable within the measurement error. 


\section{Appendix IV: Fermi's Golden Rule:}

This appendis gives the derivation of the Fermi's Golden rule. In essence, the evaluation of Fermi's Golden rule for electronic transitions coupled to the molecular vibrational degree of freedom used to develop the kinetic model closely resembles the line of reasoning employed by Persson and Persson to calculate the rate of vibrational relaxation of $\mathrm{CO}$ adsorbed on $\mathrm{Cu}(100){ }^{33}$ According to that work, the rate of transitions between the initial $|i\rangle$ and final $\mid k>$ electronic states is:

$$
\Gamma=\frac{2 \pi}{\hbar}\left|\left\langle i v\left|H^{\prime}\right| k v^{\prime}\right\rangle\right|^{2} \delta\left(\varepsilon_{i}-\varepsilon_{k}-E_{v v^{\prime}}\right)
$$

where $\mathrm{v}$ and $\mathrm{v}$ ' are the initial and final vibrational states of the molecule, $\varepsilon_{i}$ and $\varepsilon_{k}$ are energies of the initial $|\mathrm{i}\rangle$ and final $\mid \mathrm{k}>$ electronic states, $E_{v v^{\prime}}=\hbar \omega_{0} \times\left(v^{\prime}-v\right)$ is the vibrational energy change, and $\mathrm{H}^{\prime}$ is the perturbation corresponding to the Newns-Anderson type electronic ${ }^{104,105}$ Hamiltonian of the system. ${ }^{43,44}$ Designating the matrix element of the coupling between the electronic occupation of the molecular LUMO and the molecule's vibrational degree of freedom as $\lambda_{v v^{\prime}}=|<i v| H^{\prime}\left|k v^{\prime}>\right|$, Eq. (A4.1) can be re-written as:

$$
\Gamma=\frac{2 \pi}{\hbar} \lambda_{v v^{\prime}}^{2} \delta\left(E_{i}-E_{k}-E_{v v^{\prime}}\right)
$$

The total rate of vibrational energy transfer will be given by a sum of individual events shown in (A 4.2) over all possible initial and final electronic states:

$$
k_{v v^{\prime}}=\frac{2 \pi}{\hbar} \sum_{i} \sum_{k} \lambda_{v v^{2}}^{2} \delta\left(E_{i}-E_{k}-E_{v v^{\prime}}\right)
$$

To evaluate the sums in Eq. (A 4.3) the density of states, $\rho(E)$, will be defined as the number of states per energy interval such that when multiplied by the probability of occupation of a state given by the Fermi-Dirac distribution function, $f(E)$, it gives the number of occupied states in that energy range:

$$
N(E+d E)=\rho(E) f(E) d E
$$

The number of terms in the sums of Eq. (A 4.3) will be given by the number of occupied states $|i\rangle$ and empty states $\mid k>$, and thus sums in Eq. (A 4.3) can be replaced with integrals as follows:

$$
\begin{aligned}
& k_{v v^{\prime}}=\frac{2 \pi}{\hbar} \int_{0}^{\infty} d E_{i} \\
& \times \int_{0}^{\infty} d E_{k} \lambda_{v v^{2}}^{2} \rho\left(E_{i}\right) \rho\left(E_{k}\right) f\left(E_{i}\right)\left(1-f\left(E_{k}\right)\right) \\
& \times \delta\left(E_{i}-E_{k}-E_{v v^{\prime}}\right)
\end{aligned}
$$

To simplify evaluation of the integrals in Eq. (A 4.5) the coupling matrix element $\lambda_{v v}$, is assumed to not depend strongly on energy and consequently can be taken out of the integral. Similarly, 
$\rho(E)$ varies much more slowly as a function of energy than $f(E)$ and is taken as constant and is also moved in front of the integral, yielding:

$$
k_{v v^{\prime}}=\frac{2 \pi}{\hbar} \lambda_{v v^{\prime}}^{2} \rho_{i} \rho_{k} \int_{0}^{\infty} d E_{i} \int_{0}^{\infty} d E_{k} f\left(E_{i}\right)\left(1-f\left(E_{k}\right)\right) \delta\left(E_{i}-E_{k}-E_{v v^{\prime}}\right)
$$

Simplification of the delta function reduces the integrals in Eq. (A 4.6) to:

$$
k_{v v^{\prime}}=\frac{2 \pi}{\hbar} \lambda_{v v^{\prime}}^{2} \rho_{i} \rho_{k} \int_{0}^{\infty} f(E)\left(1-f\left(E-E_{v v^{\prime}}\right)\right) d E
$$

or:

$$
\begin{gathered}
k_{v v^{\prime}}=\alpha_{v v^{\prime}} \int_{0}^{\infty} f(E)\left(1-f\left(E-E_{v v^{\prime}}\right)\right) d E \\
\text { with } \alpha_{v v^{\prime}} \approx \frac{2 \pi}{\hbar} \lambda_{v v^{\prime}}^{2} \rho^{2}
\end{gathered}
$$

The integral in Eq. (A 4.8) can be taken analytically using the following algebraic equality:

$$
f(E)\left(1-f\left(E-E_{v v^{\prime}}\right)\right)=\frac{f(E)-f\left(E-E_{v v^{\prime}}\right)}{1-e^{E_{w^{\prime}} / k_{b} T_{S}}}
$$

which reduces Eq. (A 4.8) to:

$$
k_{v v^{\prime}}=\frac{\alpha}{1-e^{E_{w^{\prime}} / k_{b} T_{S}}} \int_{0}^{\infty}\left[f(E)-f\left(E-E_{v v^{\prime}}\right)\right] d E
$$

The integral in the above can be taken exactly:

$$
\int_{0}^{\infty}\left[f(E)-f\left(E-E_{v v^{\prime}}\right)\right] d E=k_{b} T_{S} \ln \frac{1+e^{-E_{F} / k_{b} T_{S}}}{e^{-E_{F} / k_{b} T_{S}}+e^{E_{v^{\prime}} / k_{b} T_{S}}}
$$

and can be approximated as:

$$
\int_{0}^{\infty}\left[f(E)-f\left(E-E_{v v^{\prime}}\right)\right] d E \approx-E_{v v^{\prime}}
$$

when:

$$
k_{b} T_{S}<<E_{F}
$$

Substituting Eq. (A 4.12) into Eq. (A 4.10) we obtain the final expression for the total vibrational energy transfer rate:

$$
k_{v v^{\prime}}=\alpha_{v v^{\prime}} \frac{E_{v v^{\prime}}}{e^{E_{w^{\prime}} / k_{k} T}-1}
$$


The condition Eq. (A 4.13) establishes the upper temperature limit of the validity of the expression Eq. (A 4.14). For example, in the case of gold $E_{F} \approx 5.5 \mathrm{eV}$ and the inequality Eq. (A 4.13) is valid until $\sim 6 \times 10^{4} \mathrm{~K}$. 


\section{References:}

(1) Zambelli, T.; Wintterlin, J.; Trost, J.; Ertl, G. Science 1996, 273, 1688.

(2) Born, M.; Oppenheimer, J. R. Annalen der Physik 1927, 84, 457.

(3) Langreth, D. C. Physical Review Letters 1985, 54, 126.

(4) Morin, M.; Levinos, N. J.; Harris, A. L. 1992, 96, 3950.

(5) Chang, H.-C.; Ewing, G. E. Physical Review Letters 1990, 65, 2125.

(6) Persson, B. N. J.; Ryberg, R. Physical Review Letters 1982, 48, 549.

(7) Ryberg, R. Physical Review B 1985, 32, 2671.

(8) Rettner, C. T.; Fabre, F.; Kimman, J.; Auerbach, D. J. Physical Review Letters 1985, 55, 1904.

(9) Nahler, N. H.; White, J. D.; LaRue, J.; Auerbach, D. J.; Wodtke, A. M. Science 2008, 321, 1191.

111.

(10) Huang, Y.; Rettner, C. T.; Auerbach, D. J.; Wodtke, A. M. Science 2000, 290,

(11) Watts, E. K.; Siders, J. L. W.; Sitz, G. O. Surface Science 1997, 374, 191.

2007, 98 .

(12) Ran, Q.; Matsiev, D.; Auerbach, D. J.; Wodtke, A. M. Physical Review Letters

(13) Wodtke, A. M.; Tully, J. C.; Auerbach, D. J. International Reviews in Physical Chemistry 2004, 23, 513.

(14) Hasselbrink, E. Current Opinion in Solid State and Materials Science 2006, 10, 192.

(15) Heilweil, E.; Casassa, M.; Cavanagh, R.; Stephenson, J. Annual Review of Physical Chemistry 1989, 40, 143.

(16) Wodtke, A. M.; Matsiev, D.; Auerbach, D. J. Progress in Surface Science 2008, $83,167$.

(17) Tully, J. C. Annual Review of Physical Chemistry 2000, 51, 153.

(18) Rahinov, I.; Cooper, R.; Matsiev, D.; Bartels, C.; Auerbach, D. J.; Wodtke, A. M. Physical Chemistry Chemical Physics 2011, 13, 12680.

(19) Bartels, C.; Cooper, R.; Auerbach, D. J.; Wodtke, A. M. Chemical Science 2011, $2,1647$.

2009.

(20) Gross, A. Theoretical Surface Sceince: A Microscopic Perspective; Springer,

(21) Gates, G. A.; Holloway, S. Surface Science 1994, 307-309, 132.

(22) Kroes, G.-J. Progress in Surface Science 1999, 60, 1.

(23) Bungaro, C.; Noguera, C.; Ballone, P.; Kress, W. Physical Review Letters 1997, $79,4433$.

(24) Billing, C. D. Dynamics of Molecule Surface Interaction; Wiley-Interscience, 1999.

(25) Matsiev, D., University of California Santa Barbara, 2007.

(26) Auerbach, D. J. Science 2001, 294, 2488.

(27) Ehrlich, G. The Journal of Physical Chemistry 1955, 59, 473.

(28) King, D. A.; Wells, M. G. Proceedings of the Royal Society of London. A. Mathematical and Physical Sciences 1974, 339, 245.

(29) Rettner, C. T.; Kimman, J.; Fabre, F.; Auerbach, D. J.; Morawitz, H. Surface Science 1987, 192, 107. 
(30) Kay, B. D.; Raymond, T. D.; Coltrin, M. E. Physical Review Letters 1987, 59, 2792.

(31) Newns, D. M. Surface Science 1986, 171, 600.

(32) Shenvi, N.; Roy, S.; Parandekar, P.; Tully, J. The Journal of Chemical Physics 2006, $125,154703$.

(33) Persson, B. N. J.; Persson, M. Solid State Communications 1980, 36, 175.

(34) Gadzuk, J. W. The Journal of Chemical Physics 1983, 79, 6341.

(35) Ran, Q.; Matsiev, D.; Auerbach, D. J.; Wodtke, A. M. Nuclear Instruments \& Methods in Physics Research Section B-Beam Interactions with Materials and Atoms 2007, 258, 1.

(36) Cho, S.-W. Journal of the Korean Chemical Society 2002, 46, 14.

(37) Katz, G.; Zeiri, Y.; Kosloff, R. Journal of Physical Chemistry B 2005, 109, 18876.

(38) Li, S. M.; Guo, H. Journal of Chemical Physics 2002, 117, 4499.

(39) Diekhoner, L.; Hornekaer, L.; Mortensen, H.; Jensen, E.; Baurichter, A.; Petrunin, V. V.; Luntz, A. C. The Journal of Chemical Physics 2002, 117, 5018. 174716.

(40) Roy, S.; Shenvi, N. A.; Tully, J. C. The Journal of Chemical Physics 2009, 130,

(41) Shenvi, N.; Roy, S.; Tully, J. C. The Journal of Chemical Physics 2009, 130, 174107.

(42) Shenvi, N.; Roy, S.; Tully, J. C. Science 2009, 326, 829.

(43) Monturet, S.; Saalfrank, P. Physical Review B 2010, 82, 075404.

(44) LaRue, J. L.; White, J. D.; Nahler, N. H.; Liu, Z.; Sun, Y.; Pianetta, P. A.; Auerbach, D. J.; Wodtke, A. M. The Journal of Chemical Physics 2008, 129, 024709.

(45) White, J. D.; Chen, J.; Matsiev, D.; Auerbach, D. J.; Wodtke, A. M. Nature 2005, $433,503$.

(46) Moore J. H., D. C. C., Coplan M. A. Building Scientific Apparatus: A Practical Guide to Design and Construction. 3rd edition ed.; Addison Wesley Publishing Company, 1989.

(47) Ran, Q.; Matsiev, D.; Wodtke, A. M.; Auerbach, D. J. Review of Scientific Instruments 2007, 78, 104104.

(48) Scoles, G. Atomic and Molecular Beam Methods; Oxford University Press, 1992; Vol. Vol. 1.

(49) Proch, D.; Trickl, T. Review of Scientific Instruments 1989, 60, 713.

(50) Rahinov, I.; Cooper, R.; Yuan, C.; Yang, X.; Auerbach, D. J.; Wodtke, A. M. The Journal of Chemical Physics 2008, 129, 214708.

(51) Herzberg, G. Spectra of Diatomic Molecules; Van Nostrand Reinhold, 1950.

(52) Demtroder, W. Laser Spectroscopy; 3rd ed.; Springer, 2002.

(53) Barrow, G. M. Introduction to Molecular Spectroscopy; McGraw-Hill Education, 1962.

(54) Banwell, C. N., McCash, E. M. Fundamentals of Molecular Spectroscopy; 4th ed.; Mcgraw-Hill College, 1994.

(55) Svanberg, S. Atomic and Molecular Spectroscopy: Basic Aspects and Practical Applications; Springer, 2004.

(56) Velarde, L.; Engelhart, D. P.; Matsiev, D.; LaRue, J.; Auerbach, D. J.; Wodtke, A. M. 2010, 81, 063106. 
(57) Lee, Y.-P.; Cheah, S.-L.; Ogilvie, J. F. Infrared Physics \& Technology 2006, 47,

227.

(58) Ladislas Wiza, J. Nuclear Instruments and Methods, 162, 587.

(59) Czanderna, A., Powell, C., Madey, T. Specimen Handling, Preperation, and Treatments in Surface Characterization; Plenum Publishers, 1998.

(60) Wodtke, A. M.; Yuhui, H.; Auerbach, D. J. Chemical Physics Letters 2005, 413, 326.

(61) Baule, B. Annalen der Physik 1914, 349, 145.

(62) Zangwill, A. Physics at Surfaces; Cambridge University Press, 1988.

(63) Green, D. S.; Bickel, G. A.; Wallace, S. C. Journal of Molecular Spectroscopy 1991, 150, 303.

(64) Rettner, C. T.; Kimman, J.; Auerbach, D. J. The Journal of Chemical Physics 1991, 94, 734 .

(65) Budde, F.; Mödl, A.; Hamza, A. V.; Ferm, P. M.; Ertl, G. Surface Science 1987, 192, 507.

(66) Sitz, G. O.; Kummel, A. C.; Zare, R. N. The Journal of Chemical Physics 1988, $89,2558$.

(67) Siders, J. L. W.; Sitz, G. O. The Journal of Chemical Physics 1994, 101, 6264.

(68) Kimman, J.; Rettner, C. T.; Auerbach, D. J.; Barker, J. A.; Tully, J. C. Physical Review Letters 1986, 57, 2053.

(69) Misewich, J.; Zacharias, H.; Loy, M. M. T. Physical Review Letters 1985, 55, 1919.

(70) Misewich, J.; Zacharias, H.; Loy, M. M. T. Journal of Vacuum Science \& Technology B: Microelectronics and Nanometer Structures 1985, 3, 1474.

(71) Korolik, M.; Suchan, M. M.; Johnson, M. J.; Arnold, D. W.; Reisler, H.; Wittig, C. Chemical Physics Letters 2000, 326, 11.

(72) Rettner, C. T.; Auerbach, D. J.; Michelsen, H. A. Physical Review Letters 1992, $68,2547$.

(73) Gostein, M.; Watts, E.; Sitz, G. O. Physical Review Letters 1997, 79, 2891.

(74) McCormack, D. A.; Kroes, G. J.; Olsen, R. A.; Groeneveld, J. A.; van Stralen, J. N. P.; Baerends, E. J.; Mowrey, R. C. Chemical Physics Letters 2000, 328, 317.

(75) McCormack, D. A.; Kroes, G. J.; Olsen, R. A.; Groeneveld, J. A.; van Stralen, J. N. P.; Baerends, E. J.; Mowrey, R. C. Faraday Discuss. 2000, 109.

(76) Somers, M. F.; McCormack, D. A.; Kroes, G. J.; Olsen, R. A.; Baerends, E. J.; Mowrey, R. C. The Journal of Chemical Physics 2002, 117, 6673.

(77) Watts, E.; Sitz, G. O. The Journal of Chemical Physics 2001, 114, 4171.

(78) Shackman, L. C.; Sitz, G. O. Journal of Chemical Physics 2005, 123.

(79) Cooper, R.; Rahinov, I.; Yuan, C.; Yang, X.; Auerbach, D. J.; Wodtke, A. M.; AVS: 2009; Vol. 27, p 907.

(80) Lykke, K. R.; Kay, B. D. Journal of Chemical Physics 1990, 92, 2614.

(81) Harris, J. Dynamics of Gas-Surface Interactions; Royal Society of Chemistry, 1991.

(82) Goodman, F. O., Wachman, H. Y. Dynamics of Gas-Surface Scattering; Academic Press, New York, 1976.

(83) Hurst, J. E.; Wharton, L.; Janda, K. C.; Auerbach, D. J. The Journal of Chemical Physics 1983, 78, 1559. 
(84) Spruit, M. E. M.; Van Den Hoek, P. J.; Kuipers, E. W.; Geuzebroek, F. H.; Kleyn, A. W. Surface Science 1989, 214, 591.

(85) Gibson, K. D.; Sibener, S. J.; Upadhyaya, H. P.; Brunsvold, A. L.; Zhang, J.; Minton, T. K.; Troya, D. The Journal of Chemical Physics 2008, 128, 224708.

(86) Watanabe, Y.; Yamaguchi, H.; Hashinokuchi, M.; Sawabe, K.; Maruyama, S.; Matsumoto, Y.; Shobatake, K. The European Physical Journal D - Atomic, Molecular, Optical and Plasma Physics 2006, 38, 103.

(87) Mortensen, H.; Jensen, E.; Diekhoner, L.; Baurichter, A.; Luntz, A. C.; Petrunin, V. V. The Journal of Chemical Physics 2003, 118, 11200.

(88) Amirav, A.; Cardillo, M. J. Physical Review Letters 1986, 57, 2299.

(89) Dixit, S. N.; Lynch, D. L.; McKoy, V.; Huo, W. M. Physical Review A 1985, 32, 1267.

(90) Rudolph, H.; Dixit, S. N.; McKoy, V.; Huo, W. M. 1988, 88, 637.

(91) Preliminary results show that $\mathrm{NO}(\mathrm{v}=3)$ is at least a factor of 10 less populated than $\mathrm{NO}(\mathrm{v}=2)$ under the experimental conditions used in this work.

(92) Zacharias, H.; Rougemont, F. d.; Heinz, T. F.; Loy, M. M. T. The Journal of Chemical Physics 1996, 105, 111.

(93) Jacobs, D. C.; Madix, R. J.; Zare, R. N. The Journal of Chemical Physics 1986, $85,5469$.

(94) Hippler, M.; Pfab, J. Chemical Physics Letters 1995, 243, 500.

(95) Cooper, R.; Rahinov, I.; Li, Z.; Matsiev, D.; Auerbach, D. J.; Wodtke, A. M. Chemical Science 2010, 55.

(96) Matsiev, D.; Li, Z.; Cooper, R.; Rahinov, I.; Bartels, C.; Auerbach, D. J.; Wodtke, A. M. Physical Chemistry Chemical Physics 2011, 13, 8153.

(97) Gates, G. A.; Darling, G. R.; Holloway, S. A theoretical study of the vibrational excitation of NO/Ag(111); AIP, 1994; Vol. 101.

(98) Bartram, M. E.; Koel, B. E. Surface Science 1989, 213, 137.

(99) Kuipers, E. W.; Tenner, M. G.; Kleyn, A. W.; Stolte, S. Chemical Physics 1989, $138,451$.

(100) Luque, J., Crosley, D.R. ; 1.5 ed.; SRI International: 1999.

(101) Krishna, V.; Tully, J. C. Journal of Chemical Physics 2006, 125.

(102) Wodtke, A. M.; Huang, Y.; Auerbach, D. J. The Journal of Chemical Physics

2003, 118,8033 .

(103) Laß, K.; Han, X.; Hasselbrink, E. 2005, 123, 051102.

(104) Anderson, P. W. Physical Review 1961, 124, 41.

(105) Newns, D. M. Journal of Chemical Physics 1969, 50, 4572. 


\section{Life Run:}

On February 3, 1984 I, Russell James Cooper, was born in Salmon Idaho, USA to Tina Olson Cooper and Michael Rhine Cooper. I am a United States citizen.

In 1998 I entered Salmon High school, where I finished my high school studies in 2002. In the same year I entered Montana State University where I studied Chemistry. During my time at Montana State I worked in the group of Professor Tim K. Minton and my bachelor's thesis focused on reactions of hyperthermal oxygen atoms with polymer surfaces. I graduated from Montana State in May 2006.

After a summer of working for Professor Minton I entered the $\mathrm{PhD}$ program at University of California Santa Barbara in September 2006. Here I started my work for Professor Alec Wodtke as well as teaching undergraduate laboratory classes for 2 years. During 2007 I oversaw the new teaching assistants. In 2008 I was awarded a PIRE-ECCI fellowship and spent five months in the group of Xueming Yang studying fast processes at surfaces. In 2010 I moved, with the rest of the Wodtke group, to Göttingen Germany. During my year in Germany I rebuilt the machine that had been used at UCSB. 


\section{Thanks:}

I would like to start by thanking Alec Wodtke and Dan Auerbach for their wonderful guidance in my wanderings through the experimental world. You both gave me a long leash to try new things, but were always there with advice and solutions when I was lost. I would also like to thank Alec for going above and beyond, as an advisor, and helping me not only scientifically, but also in my transition to adulthood. This, for anyone who knows me, was the much more difficult task and for this I am indebted.

Thanks, Tim Minton Amy Brunsvold, and Hari Upadhyaya for your ignition of my love of the lab and Tim and Hari for your continuing advice.

Thanks Dr. Igor Rahinov and Dr. Daniel Matsiev you both showed me so much about science, especially in my early days in the lab. I had a wonderful time working with both of you. What little experimental ability I have was gleaned via my time with you. You were excellent role models. Thank you, Cheng Yuan, Zhisheng, Christof, and Kai for working with me on the machine as well as the rest of the Wodtke group, both former and present.

Thank you to the staff and machine shop of both Uni Göttingen and UCSB. You were always there when I needed help. Thank you Petra for your help in teaching as well as outreach, it gave me something to look forward to every week. Special thanks to Terry and Bruce for your fast responses and wisdom. Thank you, Professor Schroeder and Inge for helping me navigate German bureaucracy.

Professor Xueming Yan, thank you for my time in China, it was wonderful. Chungyao, Zhibo, and Zefeng, it was a pleasure to work with you. Special thanks everyone who played basketball with me, especially Zhibo and Jinli. I will never forget basketball and BBQ nights.

Scott, Megan and Dallas, Rob, Julie, Dan, Bryanna the rest of the UCSB crew, you made SB a great place to live. Thanks for putting up with my craziness and for coming on all the ill-planned adventures. Bryanna thanks for always being there, whether it was dinner or backpacking, you were always down. Chris you were the best domestic partner I've ever had. Shannon Carpenter, Ka Yan and the Steves, and the CC ballers you, in your separate ways, made SB a better place. I already miss you all.

Thank you, Tim and Sonja for making me a member of your family during my time in Germany. Thanks Nina you made the German weather (more) tolerable. Thanks Florian, Jens, and the rest of my friends from Kassel it was a good year. Herzlichen Dank Dr. Herr Professor Milo Novak, du bist der beste Mann.

Thanks to all the wonderful people I've met in my life before I entered the lab and have supported me every day since: Salmon River Boys (too many to name), I loved all the skiing and boating we've done over the years. You've kept me humble in my transition to fulltime nerd. 
Kath you've been a great friend, hopefully we'll see each other more often now. Danny, Shannon, Prouds, and Big Mike, and especially Dawn Bird thanks for making college so much fun and for your continuing friendship. Big Bill and Hector, thanks for all the support and your great conversations.

Thank you, Mac, Mom, and Dad for your endless support. I love you all very much. Mom and Dad you always helped me stay on the straight and narrow and introduced me to many things in my life. Special thanks to you, Mom, as you will probably be the only person who ever reads this entire thesis. 